\title{
Novel Magnetic Resonance Acquisition and Processing Strategies for Biological Tissue Characterisation
}

\author{
By \\ Fangrong Zong
}

\begin{abstract}
A thesis
submitted to the Victoria University of Wellington

in fulfilment of the requirements for the degree of

Doctor of Philosophy

in Physics.
\end{abstract}

Victoria University of Wellington

The MacDiarmid Institute for Advanced Materials and Nanotechnology 2016 



\begin{abstract}
Proton magnetic resonance techniques have become indispensable for characterising tissues non-invasively. These methods provide abundant information regarding metabolism, morphology and histology of the sample under study. While these techniques were more expensive in the past compared to radioactive methods, modern advances in hardware and methodology provide the potential to use magnetic resonance systems more efficiently and widely. In this context, this thesis explored innovative magnetic resonance technologies from three independent perspectives which are suitable for tissue characterisation, utilising techniques from a wide range of disciplines including physics, engineering, biology and medical sciences.
\end{abstract}

One strategy relates to compressed sensing magnetic resonance imaging, seeking to recover detailed features at high undersampling rates. A data-adaptive sparse transform facilitated by principal component analysis was introduced as an alternative to the conventional pre-defined sparse transform. Moreover, the principal component analysis was used in a recognition algorithm for the reconstruction of undersampled data. The performances of these approaches were studied in cases of localised changes in the acquired images. The results demonstrated that the recognition reconstruction algorithm performed better than wavelet compressed sensing. This progress can be utilised to accelerate current state of the art imaging protocols at high magnetic field strengths. Furthermore, the prior knowledge contained in high resolution databases may enhance imaging capabilities of technologies at low magnetic field strengths.

A second approach exploits nuclear magnetic resonance diffusion contrast instead of contrast agents for tissue characterisation. Microstruc- 
tural information and global fractional anisotropy can be obtained from diffusion-diffusion correlation spectroscopy via a novel multi-dimensional gradient scheme. The concept was validated by random walk simulations and experiments of biological samples. Both correlation maps and global fractional anisotropy of in vitro healthy and tumour-bearing mouse brains were found to be different, thus providing a potential application of the proposed scheme in diffusion oncology.

In addition, a threshold algorithm on the selection of a region of interest was implemented to minimise inter-observer variations. This technique was applied to a pilot study of diffusion weighted imaging data which were acquired from patients after $\mathrm{x}$-ray mammography indicated lesions. The statistical analysis revealed an optimal threshold similar to values commonly used in positron emission tomography. Apart from selecting regions automatically, various data processing methods were implemented and compared with each other regarding their diagnostic accuracies. This field study provides opportunities for standardising procedures in diffusion weighted mammography, which may be integrated into clinical analysis in the future. 


\section{Acknowledgements}

I have been so fortunate to conduct doctoral research that fits my taste, which has led my life in New Zealand very rewarding and enjoyable. The supports from my colleagues, friends and family during these three years have made this thesis come true.

To my primary supervisor, Dr. Petrik Galvosas, thank you for taking and guiding me since the first day we met in Wellington airport. Thank you for introducing me everything elementary for research, including NMR physics, pulse sequence programming, academic writing and more importantly, presenting research to general audiences. I cannot forget the time we were sitting together to draft papers. The connection you have made with DKFZ and the conferences' opportunities you provided have benefited me more than I thought. Your continuous supports in my application for grants, scholarships and visa have made me progressing without being stuck. Danke sehr, Petrik!

To Dr. Ian Hermans and Dr. Lindsay Acelet, thank you for providing the delicate animal tissues used in my research. No experimental results can be possible without your unconditional help. Thank you, Ian, for your very attentive co-supervising and immediate support whenever I needed.

To Dr. Frederik Laun, Dr. Sebastian Bilpauspt, Lars Muller and Kerstin Damberg, thank you for making me much closer to MRI scanners and routine examinations. Frederik, thank you for teaching me the clinical breast MRI and giving me useful suggestions on my research. Your extensive intuition in diffusion equations has always motivated me. 
Gratefully, I have been studying in a lively and international environment led by Petrik, the NMR lab in Laby building. Thank Dr. Marcel Nogueira d'Eurydice for instant help in image reconstruction and modelling; Dr. Sergei Obruchkov for great assistance in imaging acquisitions and valuable feedback on my research; Dr.Bradley Douglass for debugging pulse sequences; Dr. Stefan Hertel for sharing essential experimental skills and purchasing capillaries; Dr.Tim Brox for improving my language skills, offering templates for grant applications, and sharing thesis-writing experience; Mr. Phillip Luey for proofreading my manuscript and offering more help in preparing the sample. It has been a much more meaningful life to stay with you all, especially when we had cake talks and happy hours.

I would also like to thank enormous supports from academic and general staff in Victoria University. I especially take this chance to thank Dr. Robin Dykstra, as the project principal investigator, for your persistence to my thought and the financial support from the grant. Thank Assoc. Prof. Michele Governale for providing an one-year Mathematica licence, Prof. Uli Zuelicke for supports in scholarship applications, and Dr. Paul Teal for fruitful discussions on the mathematics.

Besides, I have met so many precious post-docs and PhD students in Wellington. Thank Dr. Bridget Brox, Dr. Kai Chen, Paige Wong, Cong Zeng, Dr. Hanyue Zheng, and Dr. John Zhen who have been huge supporters during the stressful year.

Finally, I want to express my sincere thanks to my family. My parents have consistently been supportive of my career with endless love. My younger sister has been working as an accountant in a company, always encouraging me when I feel lonely. The deepest thank is given to Dr. Dr. Huabing Liu, being the man who has always accompanied me when I'm in the shadow, who has undoubtedly supported me all the time, and who has built a much balanced life for me. Nothing can be achieved without your love. I dedicate this thesis to all of my family. 


\section{Contents}

List of Figures $\quad$ ix

List of Tables $\quad$ xiii

List of Symbols and Abbreviations $\quad$ Xv

1 Introduction $\quad 1$

2 Physics of MR Imaging and Diffusometry 5

2.1 Signal Evolution and Detection . . . . . . . . . . . . . . 6

2.1.1 Bloch-Torrey equation . . . . . . . . . . . . 6

2.1.2 Elementary pulse sequences . . . . . . . . . . . 8

2.2 Magnetic Resonance Imaging . . . . . . . . . . . . . . . . . . . . . . . . . . 11

2.2.1 Spatial encoding . . . . . . . . . . . . 11

2.2.2 k-space trajectory . . . . . . . . . . . . . . . 14

2.3 Magnetic Resonance Diffusometry . . . . . . . . . . . . . . 14

2.3.1 Displacement encoding and $q$-space . . . . . . . . . 14

2.3.2 Molecular diffusion . . . . . . . . . . . . . . 17

3 Advanced MR Techniques $\quad 21$

3.1 Rapid Imaging . . . . . . . . . . . . . . . . . . . . . . . . . . . . . 22

3.1 .1 Compressed sensing MRI . . . . . . . . . . . 22

3.2 Weighted Imaging Techniques . . . . . . . . . . . . . . . . . . . . . . . 31

3.2.1 Dynamic contrast enhanced MRI . . . . . . . . . . 32 
3.2.2 Diffusion imaging techniques . . . . . . . . . . 34

3.3 Two-dimensional NMR Spectroscopy . . . . . . . . . . . 43

3.3.1 Diffusion-diffusion correlation spectroscopy . . . . . 44

3.4 Principal Component Analysis . . . . . . . . . . . . . . 48

3.4.1 The procedure of 1D-PCA . . . . . . . . . . . 49

3.4.2 The procedure of 2D-PCA . . . . . . . . 50

3.5 Biological tissues studied in this thesis . . . . . . . . . . 52

3.5.1 Plant tissue - carrot . . . . . . . . . . . . 54

3.5.2 Animal tissue - mouse brain . . . . . . . . . . . 55

3.5.3 Human tissue - breast . . . . . . . . . . . 56

4 Tissue Identification by Fast Reconstruction of Highly Undersampled MRI Data $\quad 59$

4.1 Introduction . . . . . . . . . . . . . . . 60

4.2 1D-PCA Compressed Sensing . . . . . . . . . . . . . . 61

4.2.1 Methodology . . . . . . . . . . . . . 61

4.2.2 Database evaluation . . . . . . . . . . . 63

4.2 .3 Reconstructed results . . . . . . . . . . . . 66

4.3 1D-PCA Recognition Reconstruction . . . . . . . . . . . . 67

4.3 .1 Methodology . . . . . . . . . . . . . 67

4.3.2 Reconstructed results . . . . . . . . . . . . . . 68

4.4 2D-PCA Recognition Reconstruction . . . . . . . . . . . 71

4.4.1 Methodology . . . . . . . . . . . 72

4.4.2 Comparison with CS-based algorithms . . . . . . 74

4.4 .3 Image with alterations . . . . . . . . . . . 80

4.4.4 Different database .............. . . 83

4.5 Conclusions . . . . . . . . . . . . . . . . . . 84

5 Tissue Anisotropy Determination by NMR Spectroscopy 87

5.1 Introduction . . . . . . . . . . . . . . . . 88

5.2 Methodology . . . . . . . . . . . . . . 89

5.2.1 From DTI to DDCOSY . . . . . . . . . . . 89 
5.3 Simulation . . . . . . . . . . . . . . . . . 91

5.3.1 $D$ - $D$ maps with gradients along laboratory axes . . . 91

5.3.2 $D$ - $D$ maps with the new gradient scheme . . . . . . 98

5.4 Experimental . . . . . . . . . . . . . . . . . . . 101

5.5 Results and Discussions . . . . . . . . . . . . . . 103

5.5.1 Plant tissue . . . . . . . . . . . . . . . 103

5.5 .2 Animal tissues . . . . . . . . . . . . . 106

5.6 Conclusions . . . . . . . . . . . . . . . 116

6 Quantitative Characterisation of Breast Tissue - A Field Study 119

6.1 Introduction . . . . . . . . . . . . . . . . . . . . 120

6.2 Methods . . . . . . . . . . . . . . . . . . . 121

6.2.1 Participants and MR Imaging . . . . . . . . . . . 121

6.2 .2 ROI determination . . . . . . . . . . . . . . 122

6.2.3 Threshold isocontouring . . . . . . . . . . . . 123

6.2.4 Averaging measurements . . . . . . . . . . . . 123

6.2.5 Mathematical models . . . . . . . . . . . . . . . . 124

6.2 .6 Statistical analysis . . . . . . . . . . . . . . 124

6.3 Results . . . . . . . . . . . . . . . . . 125

6.3.1 Lesion information and representative images . . . . 125

6.3.2 Dependency of quantifications on thresholds, ROI types and averaging measurements . . . . . . . . 127

6.3 .3 Statistical results . . . . . . . . . . . . . . . . 130

6.4 Discussion . . . . . . . . . . . . . . . . . . 134

6.5 Conclusions . . . . . . . . . . . . . . . 135

7 Conclusions 137

7.1 Summary of original research in this thesis . . . . . . . . 137

7.2 Recommendations for future work . . . . . . . . . . . . . 140

7.3 Final remarks . . . . . . . . . . . . . . . . . . . . 142 
Publications

173 


\section{List of Figures}

2.1 Pictures of the $9.4 \mathrm{~T}$ system used in this thesis . . . . . . . 7

2.2 FID signal and its pulse sequence . . . . . . . . . . . 9

2.3 SE signals and the pulse sequences . . . . . . . . . . . . 10

2.4 Example of (a) the proton density MR image and (b) its $k$-space data showing the sparsity of the MR data. . . . . . . 12

2.5 Diagram of 2D spatial encoding and its $k$-space trajectory . . 13

2.6 PGSE pulse sequence based on the spin echo . . . . . . . 16

2.7 PGSE signal decay of pure water in $20^{\circ} \mathrm{C}$ (a) and the dependency of free diffusion on various temperatures (b) . . . . . 18

2.8 The dependence of displacements (a) and simulated diffusion coefficients $(\mathrm{b})$ in a cylinder. . . . . . . . . . . . 20

3.1 Example of the 1D digitised signal (a) and its frequency-

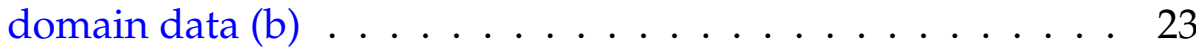

3.2 The uniform undersampling pattern in the time domain (a) and its inverse FT signal (b) . . . . . . . . . . . . . 24

3.3 The random undersampling pattern in the time domain (a) and its inverse FT signal (b) . . . . . . . . . . . . . 25

3.4 The iteration of reconstruction procedure after (a) 1, (b) 15, (c) 35 and (d) 100 steps . . . . . . . . . . . . . . 27

3.5 The undersampling mask along the phase encoding direction (a) and operated $k$-space data (b) . . . . . . . . . . . . . . 29 
3.6 The reconstructed MR image and the corresponding error image using the zero-FT technique . . . . . . . . . . . 29

3.7 Scheme of levels of wavelet decomposition (a) and sparse representation of the brain image in the wavelet domain (b) $\quad 30$

3.8 The reconstructed MR image and the corresponding error image using the wavelet-CS technique . . . . . . . . . . . 31

3.9 The three types of kinetic DCE-MRI curves . . . . . . . . . 33

3.10 Diffusion-weighted spin echo imaging sequence . . . . . . . 36

3.11 Diffusion-weighted echo planar imaging sequence . . . . . . 36

3.12 Images of the chive stalk with different $b$-values and its fitted

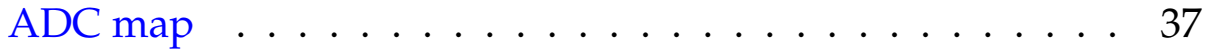

3.13 Diffusion ellipsoid models . . . . . . . . . . . . . 39

3.14 Signal attenuation curves and displacement propagator distributions at three different kurtosis values . . . . . . . . . 44

3.15 Diagram of the DDCOSY pulse sequence . . . . . . . . . 46

3.16 2D DDCOSY signal decay of the chopped chive sample and the inverted $D-D$ maps . . . . . . . . . . . . . 47

3.17 The flow chart of the 1D-PCA procedure . . . . . . . . . 51

3.18 The flow chart of the 2D-PCA procedure . . . . . . . . . 53

3.19 The cross section of a carrot . . . . . . . . . . . . . 54

3.20 Normal mouse brain anatomy . . . . . . . . . . . . 55

3.21 Normal breast anatomy . . . . . . . . . . . . . 56

4.1 The flow chart of the 1D-PCA-CS procedure . . . . . . . . 64

4.2 Carrot database evaluation. . . . . . . . . . . . . 65

4.3 1D-PCA-CS reconstruction results of case I and case II . . . . 66

4.4 The flow chart of the 1D-PCA-RR procedure . . . . . . . 69

4.5 1D-PCA-RR reconstruction results of case I and case II . . . . 70

4.6 The relationship of PSNR and number of matched images in two cases . . . . . . . . . . . . . . . 72

4.7 The flow chart of the 2D-PCA-RR procedure . . . . . . . 73

4.8 Comparison of the reconstruction methods in case I and II . 76 
4.9 Error images of the reconstruction methods in case I and II . 77

4.10 Comparison of number of iterations . . . . . . . . . . 79

4.11 The relationship between PSNR values and sampling rates . 80

4.12 Comparison of the reconstruction methods in case III . . . . 81

4.13 Error images of the reconstruction methods in case III . . . . 81

4.14 The relationship between PSNR values and sampling rates for the altered image . . . . . . . . . . . . . 82

4.15 Reconstructed results in the brain database . . . . . . . . 85

5.1 Diagram of the new gradient schemes . . . . . . . . . . 90

5.2 Simplified DDCOSY pulse sequence . . . . . . . . . . . . 93

5.3 Microscopically isotropic system and its corresponding $D-D$ map, modelled by bead pack . . . . . . . . . . . . 95

5.4 Macroscopically isotropic system and its corresponding $D-D$ map, modelled by randomly oriented fibres . . . . . . . . . 96

5.5 Macroscopically anisotropic system and its corresponding

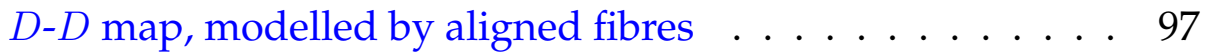

5.6 Macroscopically anisotropic system and its corresponding $D$ $D$ map, modelled by fibres with alignment degree randomly distributed between 0 and $90^{\circ} \ldots \ldots$. . . . . . . . 97

5.7 Aligned fibres in the symmetric cylinder volume and its DDCOSY simulations. . . . . . . . . . . . . . . . 99

5.8 Cross-section image of the plant tissue and the DDCOSY results . . . . . . . . . . . . . . . . . . . . . . 104

5.9 The histogram of FA values calculated from DTI results in the carrot. . . . . . . . . . . . . . . . 106

5.10 Cross-section image of the healthy brain and the DDCOSY results . . . . . . . . . . . . . . . . . . 107

5.11 The histogram of FA values calculated from DTI results in the healthy brain . . . . . . . . . . . . . . . 108

5.12 Cross-section image of the tumour-bearing brain and the DDCOSY results. . . . . . . . . . . . . . . . . . 110 
5.13 The histogram of FA values calculated from DTI results in the tumour-bearing brain . . . . . . . . . . . . . 111

5.14 The histogram of FA values of the isolated tumour part from the tumour-bearing brain. . . . . . . . . . . . . . . 112

5.15 Cross-section image of another healthy brain and the DDCOSY results . . . . . . . . . . . . . . . . . . . . 114

5.16 Signal decays before and after the additional noise. . . . . . 116

5.17 The $D$ - $D$ map after the extra noise $\ldots \ldots$. . . . . . . 117

6.1 DCE-T1WI and DWI at $b$-value of $1.5 \times 10^{-9} \mathrm{~m}^{2} / \mathrm{s} \ldots \ldots 126$

6.2 Data evolution for Npixels, ADC, $f, D$ and $K$ values of a benign lesion as a function of thresholds . . . . . . . . . 128

6.3 Data evolution for Npixels, ADC, $f, D$ and $K$ values of a benign lesion as a function of thresholds . . . . . . . . . . 129

6.4 Averaged reading differences from the two independent radiologists . . . . . . . . . . . . . . . . 130

6.5 The relationship of the AUC values with different thresholds 131

6.6 Boxplots of calculated ADC, $f, D$ and $K$ values . . . . . . . 132 


\section{List of Tables}

4.1 PSNR and SSIM values by using various reconstruction algorithms . . . . . . . . . . . . . . 76

6.1 ADC values and the corresponding sensitivities and specificities in malignant and benign lesions with a threshold of 0.85 by using three measurement methods. . . . . . . . . 133 


\title{
List of Symbols and
}

\section{Abbreviations}

\author{
$\mathrm{I}_{\mathrm{PCA}} \quad$ Reconstructed image by using PCA \\ $f\left(\mathbf{D}_{1}, \mathbf{D}_{2}\right) \quad$ 2D probability of the joint occurrence of $\mathbf{D}_{1}$ and $\mathbf{D}_{\mathbf{2}}$ \\ $\alpha \quad$ Tip angle \\ $\delta \quad$ Threshold applied on the Euclidean distance \\ $\epsilon \quad$ Iteration threshold \\ $\gamma \quad$ Gyromagnetic Ratio, $2 \pi \times 42.56 \mathrm{MHz} / \mathrm{T}$ for ${ }^{1} \mathrm{H}$ \\ $\hat{x} \quad$ Estimated 1D-signal \\ $\kappa \quad$ Permeability \\ $\lambda \quad$ Mean eigenvalue of diffusion tensor \\ $\Lambda \quad$ Eigenvalue matrix \\ $\mathbf{B}_{0} \quad$ Static magnetic field \\ $\mathbf{B}_{1} \quad$ RF field \\ D Diffusion tensor \\ $\mathrm{D}_{\mathrm{B}} \quad$ Database matrix
}




\begin{tabular}{|c|c|}
\hline G & Pulsed field gradient \\
\hline I & MR image matrix \\
\hline $\mathbf{I}_{\mathrm{c}}$ & $\begin{array}{l}\text { Image constituted from the database images with the } \\
\text { corresponding normalized Euclidean distance to the un- } \\
\text { dersampled image }\end{array}$ \\
\hline $\mathbf{I}_{\mathrm{e}}$ & Estimated MR image \\
\hline $\mathbf{K}_{\mathrm{PCA}}$ & Inverse FT of $\mathbf{I}_{\mathrm{PCA}}$ \\
\hline $\mathbf{K}_{\mathrm{u}}$ & Undersampled $k$-space data \\
\hline $\mathrm{M}_{0}$ & Thermal-equilibrium magnetisation \\
\hline $\mathrm{PC}$ & Principal Components matrix \\
\hline PJ & Projection coefficients \\
\hline $\mathbf{P J}^{\prime \prime}$ & Truncated projection coefficients \\
\hline $\mathbf{P J}^{\prime}$ & Projection coefficients of undersampled image \\
\hline $\mathbf{q}$ & Scattering wave vector \\
\hline $\mathbf{R}$ & Molecular displacement \\
\hline $\mathrm{r}$ & Molecular position \\
\hline $\mathbf{U}$ & Eigenvector matrix \\
\hline $\operatorname{Tr}(\mathbf{D})$ & The trace of diffusion tensor \\
\hline $\mathscr{F}$ & FT operator \\
\hline $\bar{P}(\mathbf{R}, \Delta)$ & Average propagator of molecular displacement at time $t$ \\
\hline$\Phi$ & Sparse Transform \\
\hline
\end{tabular}


$\Psi$

$\rho$

$\sigma$

$\mathrm{ADC}_{p}$

$\varrho$

$\vec{I}_{\mathrm{m}}$

$b$

$b_{i i}, b_{i j}$

D

$D_{0}$

$d_{e}$

$D_{1,2}^{a p p}$

$D_{i i}, D_{i j}$

f

$f_{0}^{\mathrm{LM}}$

$h_{p}$

K

$k_{B}$

M

$N_{p}$

$p$
Sampling transform

Proton density

Soft threshold

Pseudo-apparent diffusion coefficient

Smoothing parameter in ILT

Vectorised mean image of a database

Generalised gradient factor

Elements in b-matrix

Gaussian diffusion

free diffusion coefficient

Euclidean distance

Apparent diffusion coefficients obtained using DDCOSY

Elements of diffusion tensor

Perfusion factor

Larmor frequency

Plank's constant

Diffusion Kurtosis

Boltzmann constant

NMR signal

Number of tracers in random walk simulation

The number of matched images selected from a database 
$P\left(\mathbf{r}_{0}, \mathbf{r}, t\right) \quad$ Propagator of molecular at a position of $\mathbf{r}_{0}$ will be found at a position of $\mathbf{r}$ after time $t$

$T_{1} \quad$ Longitudinal Relaxation Time

$T_{2} \quad$ Transverse Relaxation Time

$T_{2}^{*} \quad$ Transverse relaxation time due to field inhomogeneities

$T_{\mathrm{E}} \quad$ Echo Time

$T_{\mathrm{R}} \quad$ Repetition Time

1D-PCA One-dimensional Principal Component Analysis

2D-FT Two-Dimensional Fourier Transform

2D-ILT Two-Dimensional Inverse Laplace Transform

2D-PCA Two-dimensional Principal Component Analysis

ADC Apparent Diffusion Coefficients

AUC Area Under Curve

BIRADS Breast Imaging Reporting And Data System

CS Compressed Sensing

DCE-MRI Dynamic Contrast-Enhanced Magnetic Resonance Imaging

DCIS Ductal Carcinoma In Situ

DKI Diffusion Kurtosis Imaging

DTI Diffusion Tensor Imaging

DWI Diffusion-Weighted Imaging 
EPI

FA

FE

FID

FLASH

FOV

IDC

ILC

ILT

MAX

MR

MRI

MRM

MRS

MSE

NMR

PCA

PFG

PGSE

PSNR

RARE
Echo Planar Imaging

Fractional Anisotropy

Fractional Eccentricity

Free Induction Decay

Fast Low Angle SHot

Field Of View

Invasive Ductal Carcinoma

Invasive Lobular Carcinoma

Inverse Laplace Transform

MAXimum pixel value of an image

Magnetic Resonance

Magnetic Resonance Imaging

Magnetic Resonance Mammography

Magnetic Resonance Spectroscopy

Mean Square Error

Nuclear Magnetic Resonance

Principal Component Analysis

Pulsed Field Gradient

Pulsed Gradient Spin Echo

Peak-Signal-to-Noise Ratio

Rapid Acquisition with Relaxation Enhancement 
RF

ROC

ROI

SE

SNR

SPAIR

SSIM

STE

T1WI

T2WI
Radio-Frequency

Receiver Operating characteristic

Region of Interest

Spin Echo

Signal-to-Noise Ratio

SPectral Attenuated Inversion Recovery

Structural SIMilarity index

STimulated Echo

$T_{1}$-Weighted Imaging

$T_{2}$-Weighted Imaging 


\section{Chapter 1}

\section{Introduction}

The nuclear magnetic resonance (NMR) phenomenon was discovered in the 1940s [1-4]. Initially, it served to study the chemical and physical properties of pure liquids and solids. After 30 years, NMR was used to study the metabolic, morphological and histological information of plants, small animals and human bodies [5-8] together with the progress of magnetic resonance spectroscopy (MRS) $[9,10]$ and the invention of magnetic resonance imaging (MRI) [11, 12]. Because of abundant water existing in tissues, hydrogen is the predominant nucleus of NMR/MRI investigations.

Although NMR/MRI is non-invasive and non-destructive, it may not be used as a preliminary diagnostic/screening method for various cancers. This is attributed to issues such as long scan time and high costs. The time-consuming acquisition reduces the amount of possible experiments/examinations within a certain time period. Moreover, the required time for imaging may be susceptible to motion from both the instruments and patients therefore will provide ambiguous results [13]. High costs mainly come from the need for large cryogen cooling systems equipped with super-conducting magnets and non-magnetic accessories, the construction of a dedicated room for housing the NMR/MRI system and the clinical use of contrast agents. Reducing acquisition time and overall ex- 
pense will be particularly beneficial, yielding to more detailed studies of biological tissues and potentially increasing patient throughput in hospital.

Recent efforts have resulted in faster MRI data collection without degrading the quality of the images. One such example is parallel MRI [1417], which can reconstruct the object based on the spatially distributed data acquisition with the aid of independent multichannel coils. This uses sophisticated radio-frequency $(\mathrm{RF})$ hardware with the corresponding acquisition-control software [18], thus the total expense of parallel MRI may offset the gain of providing shorter scan time. As an alternative, magnetic resonance (MR) images can be obtained by random undersampling $k$-space with a suitable reconstruction procedure [19-22], which is known as the compressed sensing (CS) framework. The use of CS in MRI was proposed in 2007 [23, 24], and has been successfully demonstrated in imaging various tissues, such as heart, brain, and breast $[23,25,26]$. CS requires no upgrade of the existing MRI system, thus there is no additional hardware cost to apply this framework.

While technologies like CS may help to reduce the experimental time, other methods may be developed to reduce costs. Contrast agents are commonly administrated into human body to increase the differentiation of NMR responses between tissues that are imaged simultaneously [27]. However, these expensive substances may be unnecessary if other methods can yield a sufficient contrast. Techniques such as magnetisation transfer and chemical exchange have been developed to assist this situation [28, 29]. Another promising way is to utilise molecular random motion as an inherent contrast agent, the speed of which is controlled by the microscopical structure of the underlying tissues, such as the cellular size, density, membrane boundary and fibrous construction [30]. Therefore, the change of this speed in tissues is thought to be an indication of the biophysical and physiological states [31]. For instance, cells in malignant breast tumours are densely packed. Thus water motion is hindered to a large degree [32]. NMR is an inimitable technique to quantitatively measure the distance 
of this random motion by utilising a diffusion-sensitising gradient [33]. Additionally, the stereoscopic structure of the tissue can be disclosed by applying the gradient along different directions [34].

In spite of the aforementioned progress, the application of these advanced techniques into biological tissue characterisation has been challenged in recent years. For instance, issues related to the required minimal sampling rate in the current CS frameworks were reported [35]. However, the cost of acquisition speed up is increased reconstruction time, requiring advanced computing hardware [36]. Diffusion imaging methods suffer from hardware, methodological and practical limitations [37-39]. Such weaknesses motivate one to develop alternative approaches that offer less instrumental and operational constraints to investigate the complex structures of tissues.

This thesis improves NMR methodologies for tissue characterisation through the incorporation of pattern recognition algorithms with MRI reconstruction and the development of new pulse sequence in NMR diffusometry. Furthermore, the practical issue of observer variabilities in clinical research is also addressed in this thesis.

Before going into the original contribution of this thesis, Chapter 2 provides a brief introduction of NMR and MRI fundamentals. Starting from Bloch-Torrey equations, it introduces how an NMR signal can be detected, and ends with how spatial and displacement encoding can be achieved by using pulsed field gradients.

Chapter 3 reviews more advanced NMR/MRI methods, necessary background for the subsequent chapters, ranging from the application of the CS framework into MRI to the investigation of more complex behaviour of water movements in tissues. The general morphological and histological information of the biological samples studied in this thesis is given at the end of this chapter.

From Chapter 4, the original work of this thesis is introduced. Chapter 4 elucidates three new MRI reconstruction algorithms based on CS theory. 
The motivation of using pattern recognition algorithms is presented at the beginning of this chapter, and the comparison between different algorithms is made at the end of the chapter.

In Chapter 5, an innovative experimental design is suggested based on a two-dimensional (2D) NMR spectroscopy method, in order to quantitatively evaluate orientation-dependent diffusion that was only accessible by imaging techniques. The feasibility of this methodology is addressed by a numerical simulation of aligned fibres and experiments of three biological tissues. The values obtained by the proposed approach are compared with imaging results.

Chapter 6 describes a data-driving tactics to reduce the differences of diffusion parameters read by individual radiologists in the field of MR mammography. In the meantime, it assesses the diagnostic accuracy of three averaging measurements. The optimal diffusion parameter and averaging measurement in this particular study are given at the end of this chapter.

As a closing remark, Chapter 7 summarises the original work contained in the previous chapters and discusses the possible further developments of these methodologies. 


\section{Chapter 2}

\section{Physics of MR Imaging and Diffusometry}

The inherent description of NMR phenomenon is quantum mechanics, on the other hand, it is reasonable to explain the evolution of the NMR signal by using classical physics. This chapter will give the necessary materials based on the classical description for the subsequent chapters, including the well-known Bloch-Torrey equation, elementary pulse sequences and encoding methods. More complete and advanced discussions based on the quantum physics can be found in textbooks such as Ref. [40, 41]. 


\subsection{Signal Evolution and Detection}

In order to generate an NMR signal, it is essential to place atomic spins (i.e. nuclei) into a static magnetic field $\mathbf{B}_{0}$, denoted as $\left[0,0, B_{0}\right]^{\mathrm{T}}$. This magnetic field polarises the spins (mostly referred to ${ }^{1} \mathrm{H}$ in tissues), resulting in a net magnetisation $\mathbf{M}_{0}$, denoted as $\left[0,0, M_{0}\right]^{\mathrm{T}}$. This magnetisation precesses freely around $\mathbf{B}_{0}$ with a Larmor frequency $f_{0}^{\mathrm{LM}}[2,3]$

$$
f_{0}^{\mathrm{LM}}=\frac{\gamma}{2 \pi} B_{0}
$$

where $\gamma$ is the gyromagnetic ratio $\left(2 \pi \times 42.56 \mathrm{MHz} / \mathrm{T}\right.$ for $\left.{ }^{1} \mathrm{H}\right)$. The main instrument that is used in the thesis is a $9.4 \mathrm{~T}$ MR system, which has a nominal Larmor frequency of $400 \mathrm{MHz}$. The configuration of the 9.4 T MR system is shown in Figure 2.1.

The value of this magnetisation under the condition of thermal equilibrium is proportional to the static field strength which can be described by [41]

$$
M_{0}=N_{0} \frac{\gamma^{2} h_{p}^{2} B_{0}}{16 \pi^{2} k_{B} T},
$$

where $h_{p}$ is the Plank's constant, $k_{B}$ is the Boltzmann constant, $T$ represents the temperature and $N_{0}$ stands for the number of ${ }^{1} \mathrm{H}$ in tissues. As indicated in Equation (2.2), if the tissue is measured at a middle magnetic field strength of $1.5 \mathrm{~T}$ (which is normally used in a clinical setting), the magnetisation is 5-fold less than that is measured at the $9.4 \mathrm{~T}$ magnetic field.

\subsubsection{Bloch-Torrey equation}

Besides the static field, the generation of the NMR signal requires an oscillating field $\left(\mathbf{B}_{1}\right)$ with the Larmor frequency and perpendicular to $\mathbf{M}_{0}$. This is usually accomplished by applying an RF pulse emitted by an RF coil. In order to simplify the complex phenomenon of the magnetisation 


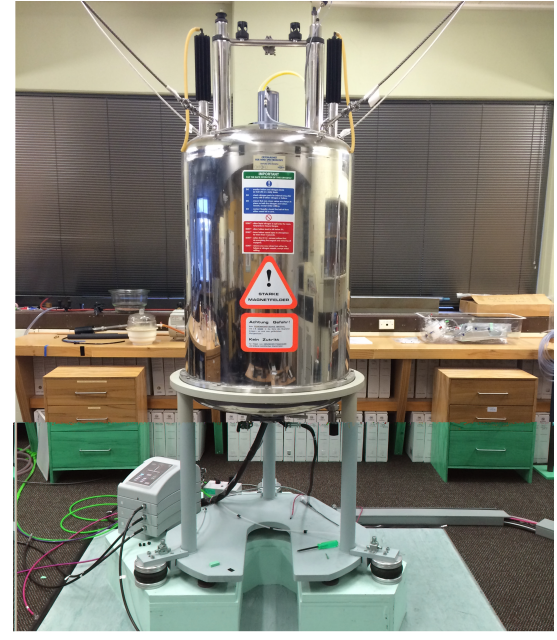

(a)

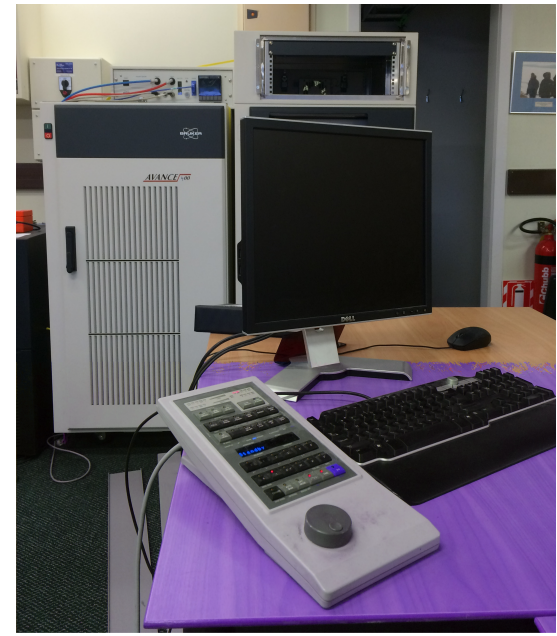

(b)

Figure 2.1: Pictures of the $9.4 \mathrm{~T}$ system (Bruker Billerica, Massachusetts, USA) used in this thesis. (a) Magnet; (b) Spectrometer and other peripherals.

during and after an RF pulse, a rotating frame of reference is used to observe the evolution, oscillating with the Larmor frequency relative to the laboratory coordinates. Therefore, $\mathbf{B}_{1}$ and $\mathbf{M}_{0}$ appear stationary in this frame. The time evolution of the magnetisation $\mathbf{M}(t)\left(=\left[M_{x}, M_{y}, M_{z}\right]^{\mathrm{T}}\right)$ interacted with an external magnetic field in the rotating frame of reference $\left(\mathbf{B}(t)=\mathbf{B}_{1}(t)\right)$ can be described by using the Bloch-Torrey equation $[2,42]$ :

$$
\frac{d \mathbf{M}(t)}{d t}=\gamma \mathbf{M}(t) \times \mathbf{B}(t)-\left[\begin{array}{c}
M_{x} / T_{2} \\
M_{y} / T_{2} \\
\left(M_{z}-M_{0}\right) / T_{1}
\end{array}\right]+\nabla \cdot \mathbf{D} \nabla \mathbf{M}(t)
$$

In Equation (2.3), $T_{1}$ describes the polarisation (i.e. recovery) of the longitudinal component and $T_{2}$ characterizes the dephasing (i.e. phase lost) of the transverse magnetization components. Both $T_{1}$ and $T_{2}$ can be referred to as a "relaxation time", with $T_{1}$ the longitudinal relaxation time and $T_{2}$ the transverse relaxation time [43], which are frequently used to differentiate between types of tissues. However, the exact values vary with the strength 
of the applied magnetic field. For instance, $T_{2}$ of a tissue ranges from $30 \mathrm{~ms}$ to $300 \mathrm{~ms}$ and $T_{1}$ from $100 \mathrm{~ms}$ to $1.5 \mathrm{~s}$ at a typical $1.5 \mathrm{~T}$ MR scanner [44-46]; however, if measured at a $9.4 \mathrm{~T}$ scanner, $T_{2}$ may be halved while $T_{1}$ may be doubled [47, 48]. $\mathrm{D}$ is the diffusion tensor of water molecules [49]. If external or background gradient is absent, $\nabla \cdot \mathbf{D} \nabla \mathbf{M}(t)$ will equal to 0 . Moreover, strictly speaking, it is independent of NMR because it reflects the nature of molecules undergoing thermal motion. Further discussion of $\mathrm{D}$ will be shown in Section 3.2.2.

Equation (2.3) is a differential equation. Thus, the solution of it is dependent on the specified initial and boundary conditions. However, the above equation can be simplified in particular cases. For instance, the relaxation and diffusion mechanisms are typically neglected during an RF pulse, meaning that only the term of $\gamma \mathbf{M}(t) \times \mathbf{B}(t)$ in Equation (2.3) is left. The assumption is valid if the pulse duration is short enough (on the order of $\mu \mathrm{s})$. Therefore, it is simply illustrated by "nutation": the $\mathbf{B}_{1}$ field rotates $\mathbf{M}_{0}$ away from the original axis ( $z$ in this thesis) with a tip angle $\alpha$. It is determined by $\gamma, B_{1}$ and pulse duration:

$$
\alpha=\gamma \int_{0}^{\tau} \mathbf{B}_{1}(t) d \tau
$$

and normally used to name the RF pulse, e.g. an RF pulse that with a tip angle of $90^{\circ}$ is known as $90^{\circ}$ pulse.

After excited by the RF pulse, the rotated magnetisation will precess around the static field due to the relaxation and diffusion mechanisms $(\gamma \mathbf{M}(t) \times \mathbf{B}(t)=0)$. This precession creates a changing magnetic flux, which in turn induces a changing voltage in a receiver coil. This voltage is the detected NMR signal that is used for further analysis in many applications.

\subsubsection{Elementary pulse sequences}

Usually not only one RF pulse but a series of RF pulses is applied in an NMR experiment. The timing of the series is referred to as "pulse 
sequence". By combing RF pulses with different tip angles at particular time points, individual relaxation and diffusion mechanisms can contribute to the NMR signal. Two examples that are elements for many advanced pulse sequences are shown in the following sections.

Free Induction Decay (FID) refers to an electromagnetic signal detected shortly after one $90^{\circ}$ pulse $[4,50]$. The pulse program and the acquired FID signal are shown in Figure 2.2.

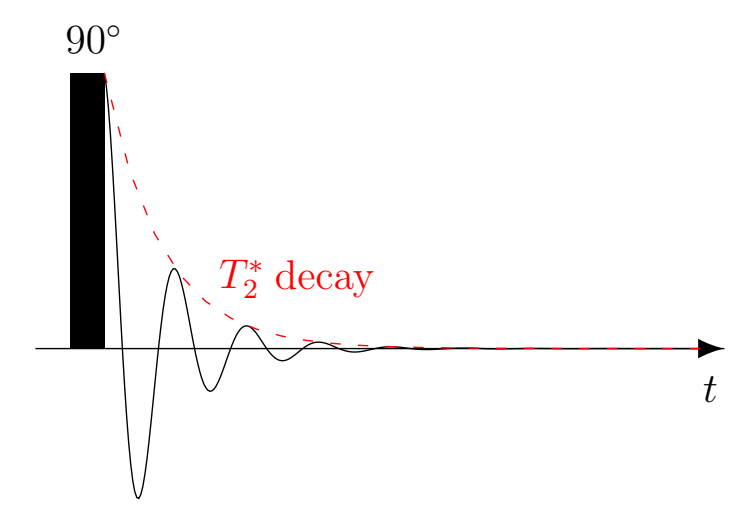

Figure 2.2: FID signal and its pulse sequence.

FID signals are used commonly in MRS to study the chemical shifts of materials. This sinusoidal signal decays exponentially with a time constant of $1 / T_{2}^{*}$. It is a combined effect of magnetic field inhomogeneities and intrinsic $T_{2}$ mechanism.

Spin Echo (SE) can be produced by adding either one $180^{\circ}$ pulse (i.e. Hahn echo) or two $90^{\circ}$ pulses (STimulated echo, STE) subsequently [51]. The time interval between the first two pulses needs to be equivalent to the time interval between the last pulse and the acquisition. In consequence, it compensates the attenuation due to local magnetic inhomogeneities. Through solving Equation (2.3), the signal intensity of SE in a static field 
can be described by

$$
M\left(T_{\mathrm{R}}, T_{\mathrm{E}}\right)=M_{0} \cdot\left[1-\exp \left(-\frac{T_{\mathrm{R}}}{T_{1}}\right)\right] \exp \left(-\frac{T_{\mathrm{E}}}{T_{2}}\right),
$$

where $T_{\mathrm{E}}$ and $T_{\mathrm{R}}$ are the echo time and repetition time, respectively. By manipulating $T_{\mathrm{R}}$ and $T_{\mathrm{E}}$, the relaxation-dependence of the acquired signal can be modulated. For example, when $T_{\mathrm{R}}=5 T_{1}$, the decay rate of the signal is only determined by $T_{2}$ because the exponential factor of $T_{1}$ becomes $1-\exp \left(-5 T_{1} / T_{1}\right) \approx 1$.

The pulse programs of Hahn echo ${ }^{1}$ and STE are shown in Figure 2.3, where both of them appear as back-to-back FIDs. The lost phase due to the local field inhomogeneities can be rewind after the $180^{\circ}$ pulse (or two $90^{\circ}$ pulses).

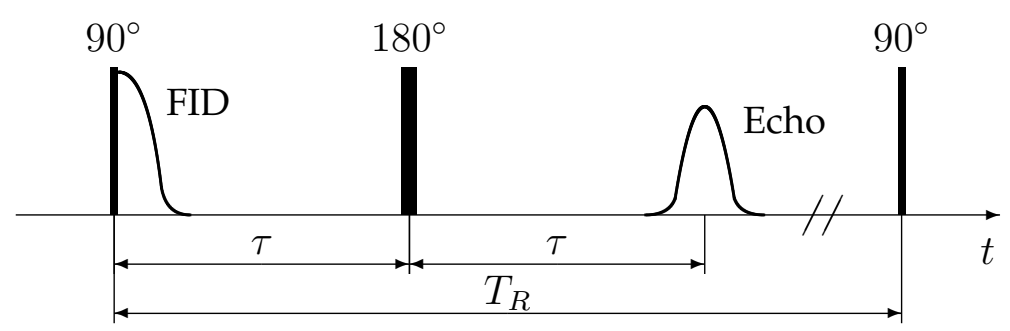

(a)

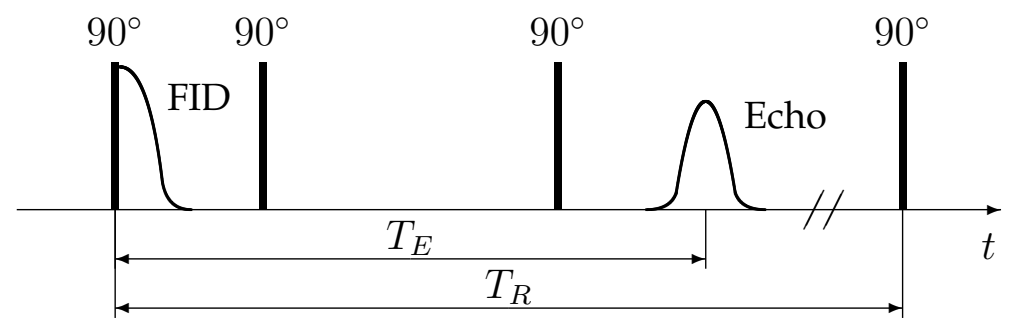

(b)

Figure 2.3: SE signals and the pulse sequences. (a) Hahn Echo; (b) Stimulated Echo. $\tau=T_{\mathrm{E}} / 2$ is the half echo time. $T_{\mathrm{R}}$ is the repetition time.

\footnotetext{
${ }^{1}$ The spin echo pulse sequence proposed by Hahn in his original paper [51] is $90^{\circ}-90^{\circ}$. The Hahn echo showed in the text is $90^{\circ}-180^{\circ}$ variant.
} 


\subsection{Magnetic Resonance Imaging}

\subsubsection{Spatial encoding}

In general, a $90^{\circ}$ hard pulse will excite all spins in tissues to the transverse plane (or $x y$-plane) without carrying any spatial distribution information. However, if a soft pulse with a limited bandwidth (e.g. a sinc shaped envelope pulse) is emitted [52,53], only magnetization at a slice location corresponding to that frequency band may be excited. By further superimposing a gradient on the static field, the field strength becomes position dependent, which makes it possible to obtain the spatial distributions of spins [11]. If only the distribution of spins in the $x y$-plane of the Cartesian coordinates is taken into account, angular Larmor frequencies $(\omega=f / 2 \pi)$ of the spins in the $x y$-plane of the Cartesian coordinates vary with their positions, which can be expressed as

$$
\omega(x, y)=\gamma B_{0}+\gamma[G(x) x+G(y) y]
$$

where $G(x), G(y)$ and $x, y$ are the field gradient strengths and spin positions along $x$ - and $y$-directions, respectively. The detected NMR signal is an integral of all spins with their own processing frequencies [12, 54]:

$$
M(t)=\iint \rho(x, y) \exp \{i \gamma[G(x) x+G(y) y] t\} \mathrm{d} x \mathrm{~d} y .
$$

Here, $\rho(x, y)$ is the spin density distribution. By introducing the concept of $k$-space $[12,55]$, Equation (2.7) can be rewritten as,

$$
M\left(k_{x},, k_{y}\right)=\iint \rho(x, y) \exp \left[i 2 \pi\left(k_{x} x+k_{y} y\right)\right] \mathrm{d} x \mathrm{~d} y .
$$

where,

$$
k_{x, y}=(2 \pi)^{-1} \gamma G_{x, y} t
$$


It is evident that $\rho(x, y)$ and $k(x, y)$ are a Fourier transform pair. By sampling in time ( $k$-space) domain in two directions and subsequently applying a two-dimensional Fourier Transform (2D-FT) [56-58] on Equation (2.8), the spatial distribution $\rho(x, y)$ can be obtained, which forms a 2D proton density MR image. An example of $k$-space map and MR image is given in Figure 2.4. As can be observed from Figure 2.4, signal intensities in the MR image are distributed in the 2D map, whereas only few non-zero points that are centred in $k$-space can be found. This characteristic makes compressed sensing idea naturally applicable to MRI [23], which will be further discussed in Section 3.1.1 of Chapter 3.

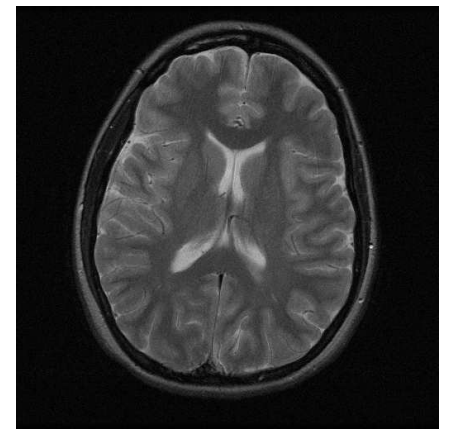

(a)

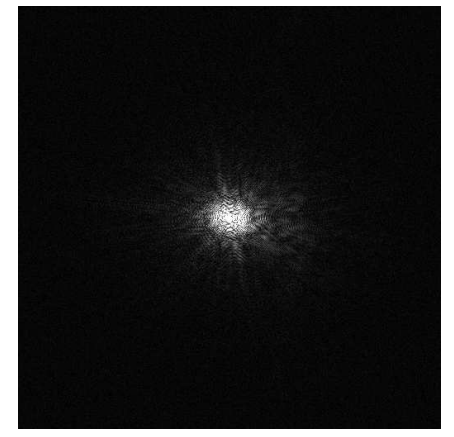

(b)

Figure 2.4: Example of (a) the proton density MR image and (b) its $k$-space data showing the sparsity of the MR data.

The 2D spatial encoding (in other words, sampling in $k$-space) includes two-directional encodings as shown in Figure 2.5. The read gradient is applied with a constant value during the acquisition (i.e. varying $t$ in Equation (2.9)) to encode frequency differences [59], and another perpendicular gradient is applied with varying the gradient strength up to its maximum $G_{\mathrm{ph}}^{\max }$ in Equation (2.9), to encode phase differences [60]. Either encoding gradient can be applied along the $x$ or $y$ axis, with individual determining 
the field of view (FOV) and the resolution in each direction [61]:

$$
\begin{aligned}
\mathrm{FOV}_{\text {freq }} & =2 \pi /\left(\gamma G_{\text {freq }} t_{\text {acq }}\right) ; \\
\mathrm{FOV}_{\text {phase }} & =2 \pi /\left(\gamma G_{\mathrm{ph}}^{\max } t_{\mathrm{ph}}\right) .
\end{aligned}
$$

Where, $G_{\text {freq }}$ and $t_{\text {acq }}$ are the strength and the duration for the frequencyencoding gradient, respectively. $G_{\mathrm{ph}}^{\mathrm{max}}$ and $t_{\mathrm{ph}}$ are the maximal strength and duration of the phase-encoding gradient, respectively. Subsequently, the resolution in each direction can be calculated by dividing the FOV with the number of acquisition points.

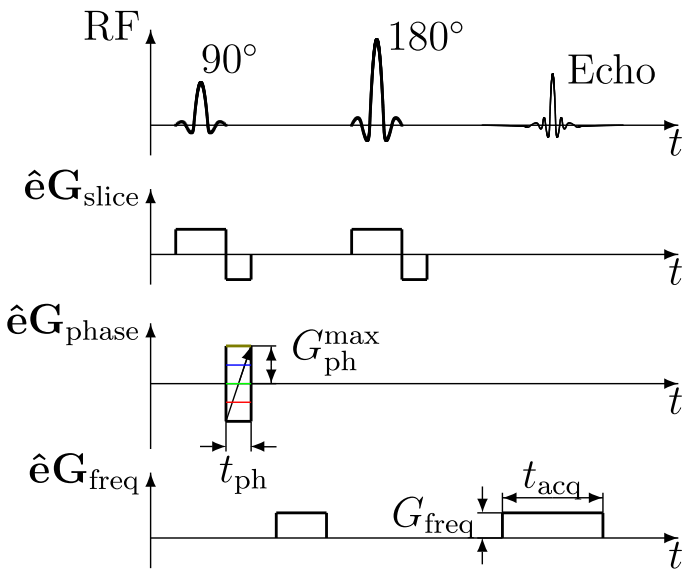

(a)

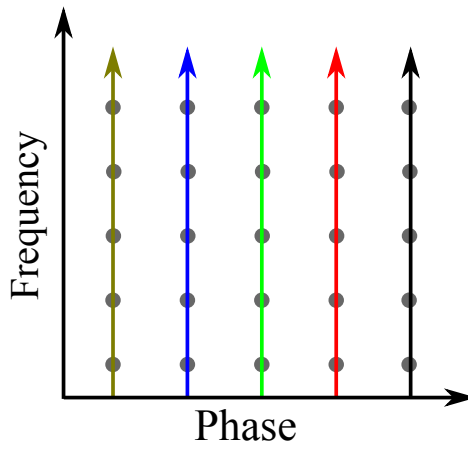

(b)

Figure 2.5: Diagram of (a) 2D spatial encoding and (b) its $k$-space trajectory. The soft RF pulses with limited bandwidth are shown at the top of the pulse sequence. $\mathbf{G}_{\text {slice}}, \mathbf{G}_{\text {phase }}$ and $\mathbf{G}_{\text {freq }}$ are the slice selection, phase encoding and readout gradients, respectively. The symbol $\hat{\mathbf{e}}$ in the front represents the unit vector in the direction of the respective gradient in the laboratory coordinate system. $G_{\mathrm{ph}}^{\max }$ and $t_{\mathrm{ph}}$ are the maximal value and duration of the phase-encoding gradient, respectively. $t_{\text {acq }}$ is the acquisition time and the duration for the frequency-encoding gradient. The phase-encoding gradient strength is stepped in the following experiment until it reaches the maximal value, which is indicated by the horizontal lines inside the gradient pulses. 


\subsection{2 k-space trajectory}

The sequence of using digitised MR signals to fill in the $k$-space is commonly known as " $k$-space trajectory". Figure 2.5 (b) depicts the $k$-space trajectory using the encoding of Figure 2.5 (a). As it fills the whole $k$-space column-by-column in a Cartesian grid, the reconstruction of the MR image only needs 2D-FT, which is simple and intuitive. However, it is sensitive to the motion of the instrument and imaged subject, which limits its use in some particular fields such as cardiac imaging and MR angiography. To overcome these issues, non-Cartestian trajectories have been designed (e.g. radial, spiral) together with non-uniform FT reconstruction [62, 63]. On the other hand, undersampling $k$-space is possible with the theoretical development of CS [23].

\subsection{Magnetic Resonance Diffusometry}

\subsubsection{Displacement encoding and $q$-space}

Additionally to acquiring proton density, $T_{1}$ and $T_{2}$ weighted values of a tissue, there exists another encoding method that has been used extensively to monitor molecular displacement in the presence of a magnetic field gradient [33,64-66]. It provides the morphological information of the intracellular and extracellular spaces [67] as well as the mobility of molecules in tissues [68].

The statistical description for molecular displacement is the probability density or propagator, which describes the chance that a molecule starts at $\mathbf{r}_{0}$ at time $t=0$ will be found at a position of $\mathbf{r}$ after time $t$. According to Fick's second law, this propagator $P\left(\mathbf{r}_{0}, \mathbf{r}, t\right)$ can be described by a partial differential equation [69], which is known as the diffusion equation:

$$
\frac{\partial}{\partial t} P\left(\mathbf{r}_{0}, \mathbf{r}, t\right)=\nabla \cdot \mathbf{D} \nabla P\left(\mathbf{r}_{0}, \mathbf{r}, t\right)
$$


If the initial condition is considered to be a Dirac delta function and the boundary conditions are $P\left(\mathbf{r}_{0}, \mathbf{r}, t\right) \rightarrow 0$ and $\mathbf{r} \rightarrow \infty$, the solution of Equation (2.10) is a Gaussian function [33, 70]:

$$
P\left(\mathbf{r}_{0}, \mathbf{r}, t\right)=\frac{1}{\sqrt{|\mathbf{D}|(4 \pi t)^{3}}} \exp \left(-\frac{\left(\mathbf{r}-\mathbf{r}_{\mathbf{0}}\right)^{\mathrm{T}} \mathbf{D}^{-1}\left(\mathbf{r}-\mathbf{r}_{\mathbf{0}}\right)}{4 t}\right),
$$

where, $|\mathbf{D}|$ is the determinant of $\mathbf{D}$.

Pulsed field gradient (PFG) or pulsed gradient spin echo (PGSE) is a unique technique that can obtain the information about the displacement propagator $P\left(\mathbf{r}_{0}, \mathbf{r}, t\right)[33,64]$. An example of the pulse sequence is shown in Figure 2.6. It is based on a spin echo pulse sequence as shown in Figure 2.3 (a) with two additional gradient pulses (aka PFG pair). They both last for a duration of $\delta$ and separated by a time interval of $\Delta$. The detected NMR signal as an ensemble average is the integral over the phase differences of all spin-carrying molecules

$$
M\left(\mathbf{G}_{\text {diff }}, \Delta\right)=\int \bar{P}(\mathbf{R}, \Delta) \exp \left(i \gamma \delta \mathbf{G}_{\text {diff }} \cdot \mathbf{R}\right) d \mathbf{R} .
$$

Here, $\mathbf{R}=\mathbf{r}-\mathbf{r}_{0}$ is the displacement, $\bar{P}(\mathbf{R}, \Delta)$ is the average propagator [71] introduced by the equation

$$
\bar{P}(\mathbf{R}, \Delta)=\int \rho\left(\mathbf{r}_{0}\right) \cdot \bar{P}(\mathbf{R}, \Delta) d \mathbf{r}_{0},
$$

where $\rho\left(\mathbf{r}_{0}\right)$ represents a probability of finding a molecule at position $\mathbf{r}_{0}$. $\mathbf{G}_{\text {diff }}$ denotes the amplitude and direction of the diffusion-sensitising gradient. The introduced " $q$-space" by Callaghan et al. [72] enables the reformulating of Equation (2.12) to be

$$
M(\mathbf{q}, \Delta)=\int \bar{P}(\mathbf{R}, \Delta) \exp (i 2 \pi \mathbf{q} \cdot \mathbf{R}) d \mathbf{R},
$$

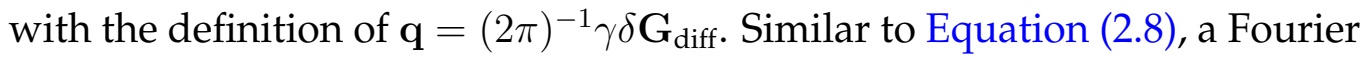


relationship is seen in Equation (2.14). Thus, signal sampling in $q$-space allows for "imaging" molecular displacements. Equation (2.14) has become the core of many newly developed PGSE-NMR techniques, such as overcoming the resolution limitations of conventional imaging methods [73, 74] or single-shot diffusion experiments [75].

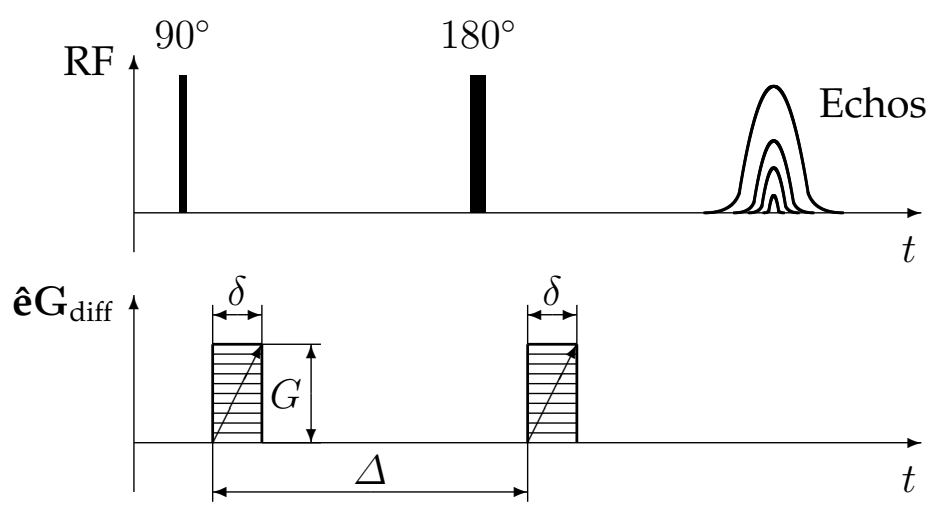

Figure 2.6: PGSE pulse sequence based on the spin echo, where $\delta$ and $\Delta$ are the gradient duration and observation time, respectively. $\mathbf{G}_{\text {diff }}$ is the displacementencoding gradient. The symbol $\hat{e}$ in the front represents the unit vector in the direction of the respective gradient in the laboratory coordinate system. The displacement-encoding gradient strength is stepped in the next experiment until it reaches the maximal value, which is indicated by the horizontal lines inside the gradient pulses.

Considering the case where the projection of the displacement on the direction of the gradient pair is applied $(\mathbf{R} \rightarrow R)$, the cumulant expansion allows replacing the integral on the right side of Equation (2.14) to be [65, 76]

$$
\int \bar{P}(R, \Delta) \exp (i 2 \pi q R) d R=\sum_{n=0}^{\infty} \frac{(i 2 \pi q R)^{n}}{n !}\left\langle R^{n}\right\rangle_{c}
$$

where, $R$ is the component of displacement along the gradient direction defined by $q$ (of which, $q$ is the amplitude). $\langle\cdot\rangle_{c}$ stands for the cumulant values of molecules. Therefore, the representation of $M(q, \Delta)$ by averaging 
phase shift leads to

$$
M(q)=M(0)\left[1-(1 / 2 !)(2 \pi q)^{2}\left\langle R^{2}\right\rangle_{c}+(1 / 4 !)(2 \pi q)^{4}\left\langle R^{4}\right\rangle_{c}+O\left(q^{6}\left\langle R^{6}\right\rangle_{c}\right)\right]
$$

where, $M(0)$ is the magnetisation acquired when no gradient is applied. $\left\langle R^{2}\right\rangle_{c}$ is the corresponding mean squared displacement. The Gaussian nature of Equation (2.11) allows the higher order term $(1 / 4 !)(2 \pi q)^{4}\left\langle R^{4}\right\rangle_{c}$ to be zero. However in some cases, the displacement propagator function may not be a Gaussian distribution, thus the higher order term is non-zero. Chapter 3 will review an advanced technique that deals with the higher order term. In the next section, a short introduction to molecular diffusion and how to measure it (aka diffusometry) via the displacement information is given.

\subsubsection{Molecular diffusion}

Molecular self-diffusion ${ }^{2}, D$, often simply called "diffusion" in NMR, refers to the process that molecules undergo a stochastic (i.e. Brownian) motion associated with thermal energy ${ }^{3}$. The Gaussianity of Equation (2.11) leads to the Einstein equation linking diffusion and mean squared displacement at a time interval of $\Delta[68]$ :

$$
D=\frac{\left\langle R^{2}\right\rangle_{c}}{2 \Delta},
$$

By substituting Equation (2.17) into Equation (2.16) and re-using the Taylor expansion for exponential representation, the signal decay equation can be re-written as

$$
M(q)=M(0) \exp \left(-4 \pi^{2} q^{2} D \Delta\right) .
$$

\footnotetext{
${ }^{2}$ This paragraph describes the free diffusion of molecules, thus the diffusion tensor is reduced to a scalar value.

${ }^{3}$ It should be mentioned that the concept of conventional diffusion holds for a time longer than 10 ps (picoseconds) [77]. "Anomalous diffusion" may be observed at times shorter than 10 ps $[78,79]$
} 
The value of $D$ can then be obtained by fitting the signal using a monoexponential model. Figure 2.7 (a) is the signal decay curve of water molecules at $20^{\circ} \mathrm{C}$ obtained by using the PGSE pulse sequence shown in Figure 2.6, the slope of which indicates molecular thermal diffusion information, which is $2.06 \pm 0.03 \times 10^{-9} \mathrm{~m}^{2} / \mathrm{s}$. Naturally, with increasing the temperature, molecules diffuse more intensively, leading to a larger diffusion coefficient value. By employing PGSE technique consecutively under different heating conditions, the dependence of diffusion coefficients on the temperatures can be obtained and shown in Figure $2.7(b)$, which is reproduced from [80]. A non-linear increasing tendency of the diffusion coefficients is seen with the increment of the temperature. For instance, the free diffusion coefficient of water increases to $2.9 \times 10^{-9} \mathrm{~m}^{2} / \mathrm{s}$ at $35^{\circ} \mathrm{C}$.

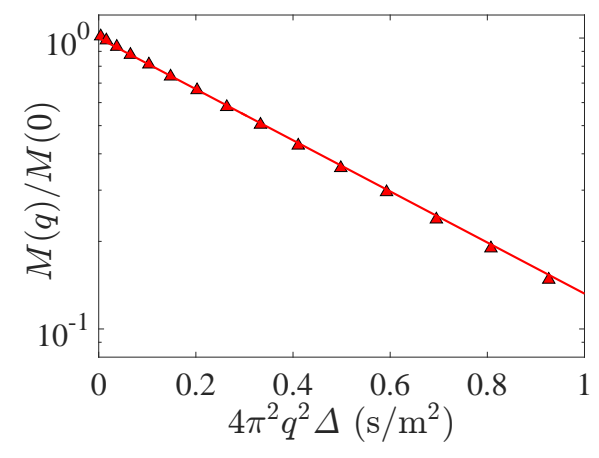

(a)

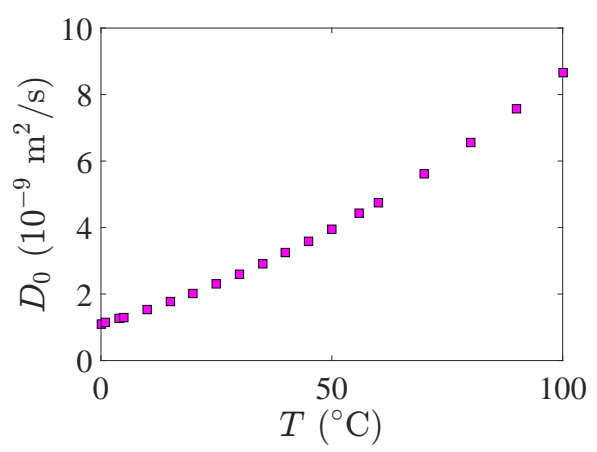

(b)

Figure 2.7: PGSE signal decay of pure water in $20^{\circ} \mathrm{C}$ (a) and the dependency of free diffusion on various temperatures $(b)$ [80].

It should be mentioned that at large time-scale (large $\Delta$ ), the mean squared displacement of molecules in tissues may be affected by the boundaries of the space, which is depicted in Figure 2.8 (a). The displacements of the water molecules increase equally in all directions in an unhindered environment when the observation time is in the short time limit. If sufficient time is allowed for the diffusing molecules to be impeded by the barrier of the space (specifically, cylinder in Figure 2.8), the displacements give 
an indication of the shape and orientation of the structure. If the barrier is impermeable, at some point, the displacements no longer increase with the observation time because the diffusing molecules are physically restricted. This leads to a fact that the derived diffusion coefficient from PGSE signal decay is time-dependent containing the geometry of the boundaries [30, 81]. In order to illustrate this, PGSE signals at various observation times by using Monte-Carlo simulations were obtained. The PFG pair was applied perpendicular to the cylinder. The calculated diffusion coefficients are shown in Figure 2.8 (b), following a decreasing pattern when the observation time is increased.

If, however, the barrier is permeable, such as cell wall in a tissue, the displacement of the molecules may be still increasing even though the observation time is long. This is because the molecules may travel into the adjacent compartment. It has been validated that the measured diffusion coefficient in a long time limit contains both information about the compartment length and the permeable property of the barrier $[82,83]$

$$
D=\frac{D_{0}}{1+D_{0} / \kappa a},
$$

where $D_{0}$ is the free diffusion coefficient of the molecules, and $\kappa$ indicates the permeability of the space boundary. This model has been used in the quantitative mapping of tissue permeability in plants [84]. More complex behaviour (e.g. non-Gaussian diffusion) will be explored in Chapter 3. 


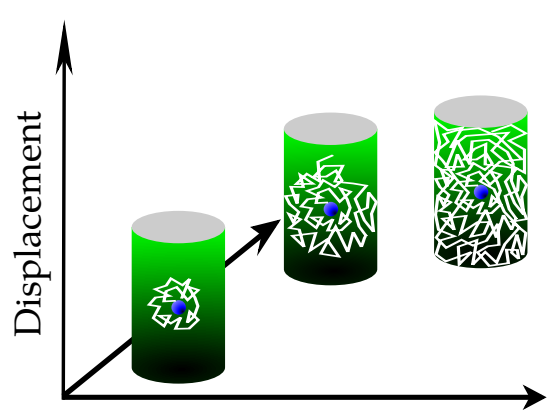

Observation time

(a)

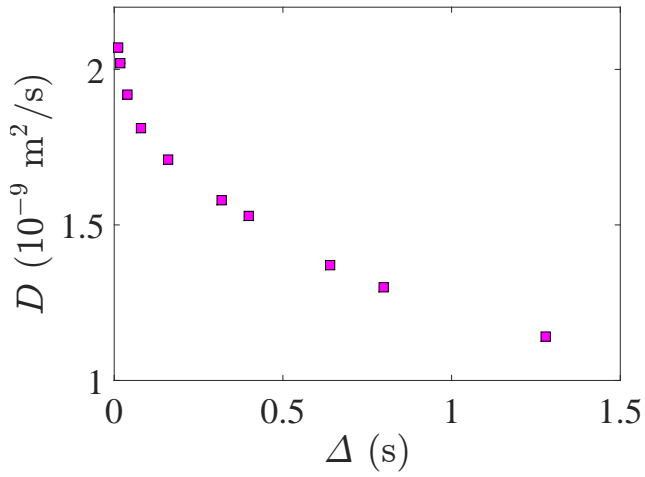

(b)

Figure 2.8: The dependence of displacements (a) and simulated diffusion coefficients (b) on the observation time in a cylinder model. The simulation was implemented by using the Monte-Carlo algorithm discussed in Chapter 5. The result shown here is for illustration purpose. White curves inside the cylinder represent the trajectories of the molecules. The displacements of the water molecules increase equally in all directions in an unhindered environment when the observation time is in the short time limit. If sufficient time is allowed for the diffusing molecules to be impeded by the barrier of the cylinder, the displacement is no longer increased. Therefore, the calculated diffusion coefficient will be decreased with the incremental observation time. It should be noted that $D$ is the measured or apparent diffusion coefficient and $\Delta$ is the "effective" diffusion time. More details will be shown in the next chapter. 


\section{Chapter 3}

\section{Advanced MR Techniques for Image Acquisition and Tissue Characterisation}

Since its invention 70 years ago, NMR has improved dramatically in the characterisation of porous media in general. Advanced techniques have revolutionised many disciplines such as material science and medicine. This chapter reviews stateof-art methods to which the thesis strongly related, ranging from rapid imaging development, the use of contrast agents, and complex diffusion behaviour to feature extraction in biological tissues. At the end of this chapter, the tissue composition, morphologies and anatomies of biological samples studied in this thesis work are briefly introduced. 


\subsection{Rapid Imaging}

As mentioned in the previous chapter, MRI acquisition is a process of sampling $k$-space data, of which the speed is limited by physical constraints. Scientists are attempting to reduce the amount of acquisition time without losing essential information. One approach is to start from reducing the number of experiments required for sampling the whole $k$-space. By designing sophisticated gradient-encoding pulse sequences, such as echo planar imaging (EPI) [85], fast low angle shot (FLASH) [86] and rapid acquisition with relaxation enhancement (RARE) [87], multiple $k$-space lines can be filled at once. Hence, the total acquisition time is largely reduced. Other approaches utilise specific hardware of multiple receiver coils, increasing the acquisition speed by a factor equal to the number of coils used [16$18,88]$. Moreover, because MRI data is redundant, it is possible to sample few points even if only one receiver coil is available, which is built on the idea of compressed sensing.

\subsubsection{Compressed sensing MRI}

CS is one of the signal processing techniques for efficiently acquiring and reconstructing signals. This section will review how it is developed in the signal processing field and how it is possible to apply the CS idea into the MRI field.

The common goal of signal processing is to capture information (e.g. frequency range) of an analogue signal from a series of digitised measurements. An early breakthrough in signal processing was the Nyquist-Shannon theorem $[89,90]$. It requires the sampling rate to be higher than the highest frequency of the signal, in order to capture all information and reconstruct the signal perfectly. The sampling rate below that criteria may lead to a coherent (aliasing) artefact, which is a superposition of shifted replicas of the true signal. As the true signal and the replicas appear the same, it is impossible to distinguish between them. 
Figure 3.1 (a) shows an intuitive example of digitised signal and its representation in the frequency domain. The time-domain dataset contains a wide range of intensities at different sampling time points. The number of sampling points is 128 in this case. However, the frequency-domain signal only consists of 5 peaks with distinct intensities.

In order to elucidate the consequence of violating the Nyquist-Shannon theorem, the signal in Figure 3.1 (a) is undersampled. A sampling rate of $25 \%$ is applied to the time-domain data, leading to a measurement illustrated in Figure 3.2 (a). This type of undersampling is commonly known as "uniform undersampling" because time for successive sampling points is identical. By applying FT, the frequency-domain signal is seen in Figure 3.2 (b). In order to relatively compare the reconstructed signal with its original data, the amplitude in Figure 3.2 (b) is amplified by a factor of $1 / 0.25=4$. It is observed that now the frequency-domain signal in Figure 3.2 (b) contains 4 replicas of the true signal in different positions and the major features shown in Figure 3.1 (a) can not be obtained from Figure 3.2 (b) using such undersampling pattern. Therefore, Nyquist-Shannon theorem needs to be satisfied if the signal is acquired in a uniform manner.

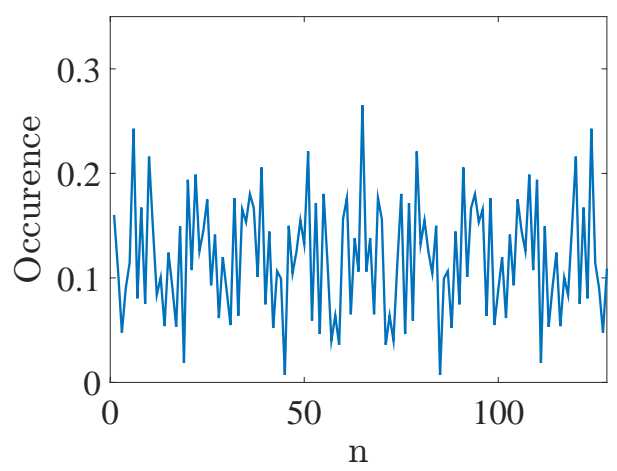

(a)

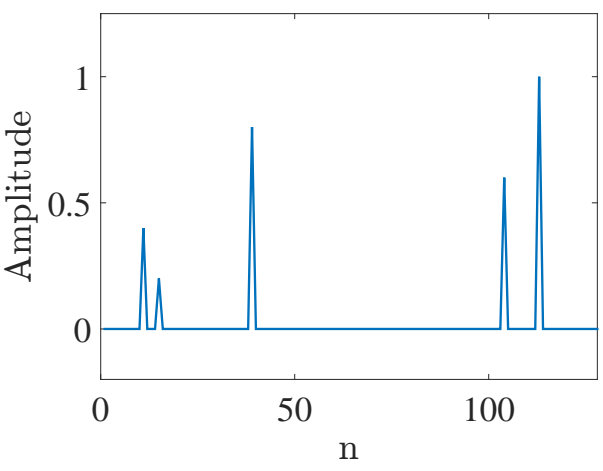

(b)

Figure 3.1: Example of (a) the 1D time-domain (digitised) signal and (b) its frequency-domain counterpart. $n$ is the sampling point.

In 2005, Candès and Romberg [19, 20, 91] found that a non-uniform 


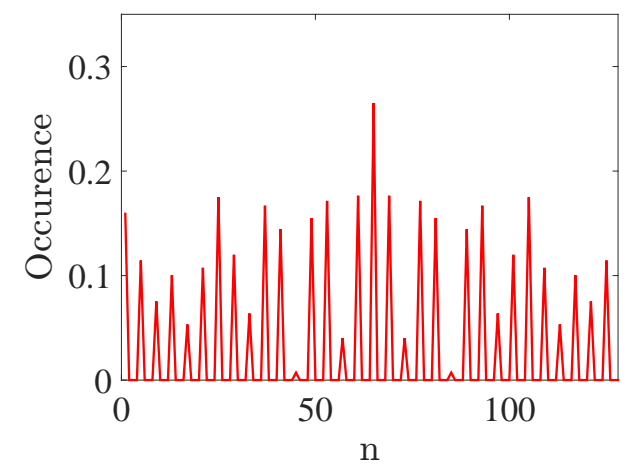

(a)

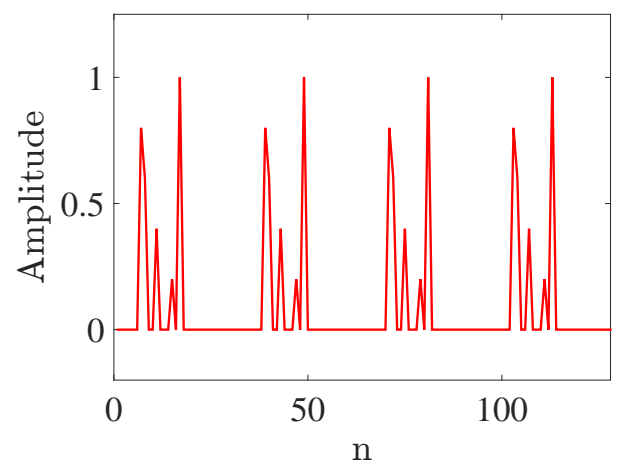

(b)

Figure 3.2: The uniform undersampling pattern in the time domain (a) and its inverse FT signal (b).

(specifically random) undersampling manner can resolve the problem of aliasing artefacts caused by the violation of Nyquist-Shannon theorem. The reconstruction exhibits incoherent artefacts that behave like (but is not) additive random noise. In fact, the artefacts are indications of the random re-distribution of signal amplitude due to this special sampling scheme. Figure 3.3 shows the random undersampling pattern of the time-domain signal in Figure 3.1 (b) and the reconstructed results via FT. Note that the amplitude of frequency-domain data is multiplied by 4 . Despite the noisy appearance, 2 peaks with relatively large amplitude stand in the same position as in the original signal. Unlike uniform undersampling where the replicas conceal the information of the original signal, random undersampling makes it possible to read some features as contained in the original peaks at first glance.

Having discussed the random undersampling strategy, the question for reconstructing the frequency-domain signal now becomes to recover the peaks that are drowned in the "noise" level. The answer is that not all types of digitised signals can be fully recovered, only which is sparse in a certain "domain" can be possibly reconstructed $[22,92]$. The "domain" is constituted of a series of orthogonal basis functions and the sparsity means 
that most of the coefficients of the orthogonal basis functions are exactly zeros (strong sparsity) or very small relative value (weak sparsity). For example, FT domain is formed by a set of complex exponential functions, and the sparsity requires the coefficients of the exponential functions to be nearly zero. In our 1D example, as can be seen in Figure 3.1, although the signal is not sparse in the time domain, the dataset in the frequency domain is sparse (only 5 out of 128 points contain non-zero intensities) which meets the requirement. Therefore, it is possible to recover the true frequency-domain signal from the random undersampled dataset via the use of FT as a sparse transform.

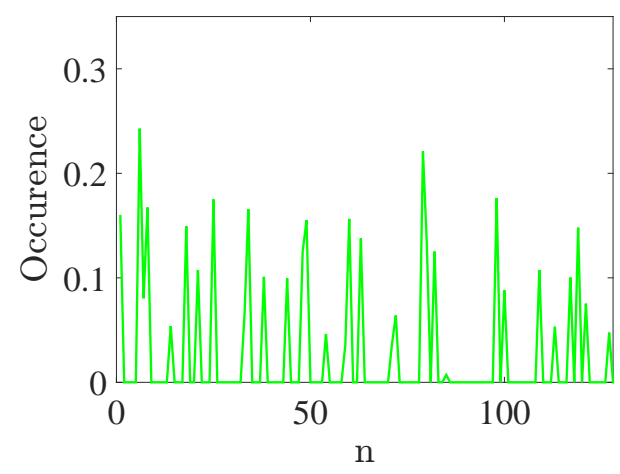

(a)

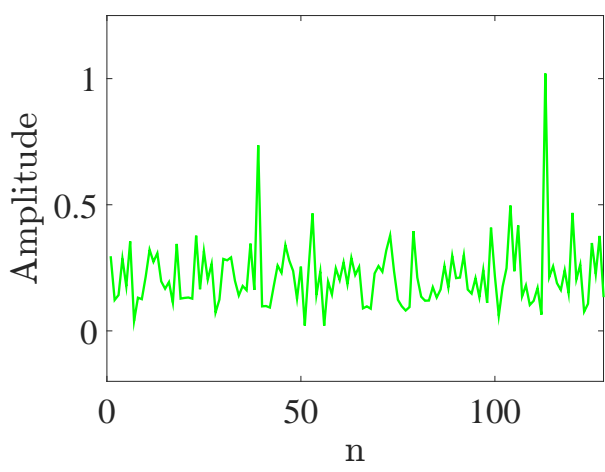

(b)

Figure 3.3: The random undersampling pattern in the time domain and its inverse FT signal. (a) Random undersampling; (b) reconstructed signal from (a) using FT where no aliasing effect is shown.

Once the sparse domain is chosen, the recovery of the signal follows a common concept via an iterative optimisation approach:

$$
\operatorname{argmin}\|\Phi \hat{x}\|_{1}, \quad \text { subject to }\|\Psi \hat{x}-y\|_{2}<\epsilon,
$$

where $\hat{x}$ and $y$ are the estimated (reconstructed) signal and measurements, respectively. $\Psi$ is the transform from the signal to measurements, and $\Phi$ is the sparse transform of the signal. $\epsilon$ is the threshold to control data 
consistency. $\|\cdot\|_{1}$ and $\|\cdot\|_{2}$ are the $l_{1}$ norm (sum of the absolute elements) and the $l_{2}$ norm (sum of squared elements), respectively. Equation (3.1) is known as " $l_{1}$ norm minimisation" which is now commonly used in CS. Other approaches, such as " $l_{0}$ minimisation", are also explored in CS in order to recover the signal [21]. Many algorithms, such as Bregmen iteration [93], conjugate gradient method [23, 24] and thresholding [94, 95], are demonstrated feasible to solve the optimisation function as described in Equation (3.1).

An example of using "soft threshold" to recover the signal based on the measurements in Figure 3.3 (a) can be described by

$$
\Phi \hat{x}_{n+1}=\left(\Phi \hat{x}_{n}\right) \cdot\left(1-\frac{\sigma}{\Phi \hat{x}_{n}}\right) .
$$

Where $\sigma$ is a threshold value applied on the coefficients of the FT domain, and $n$ is the number of iterations. Any value smaller than $\sigma$ will be treated as zero. $\Phi \hat{x}_{n+1}$ represents the updated coefficients after each iteration. The reconstruction results from different iterative steps that are illustrated in Figure 3.4. It is seen that the noise level is gradually suppressed and the information of the true signal in the frequency domain, including peak positions and relative intensities, is gradually recovered with the iteration.

It may be argued that CS violates the Nyquist-Shannon theorem. However, this is a misconception because CS depends on the sparsity of the signal and not its highest frequency. Moreover, the Nyquist-Shannon theorem provides sufficient, but not necessary conditions for guaranteeing perfect reconstruction. A sampling method that is fundamentally different from classical fixed-rate sampling cannot "violate" the theorem.

The use of CS in MRI starts 2 years after the theory of CS was complete since the idea of CS is naturally applicative to MRI [23]. CS-MRI addresses the issue of long scan time by sampling much fewer points in $k$-space. In the context of 2D MRI acquisition and reconstruction, Equation (3.1) can be 


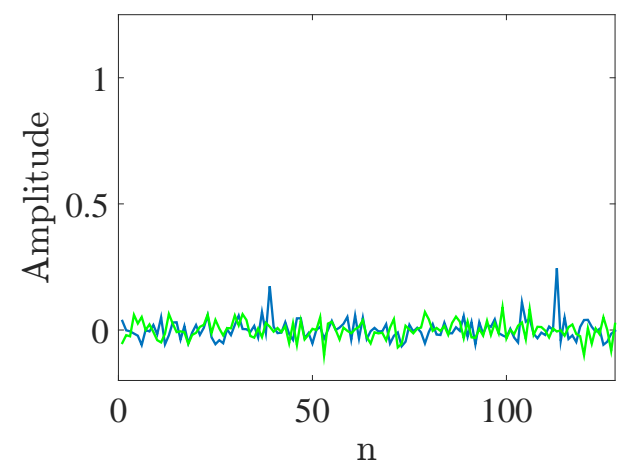

(a)

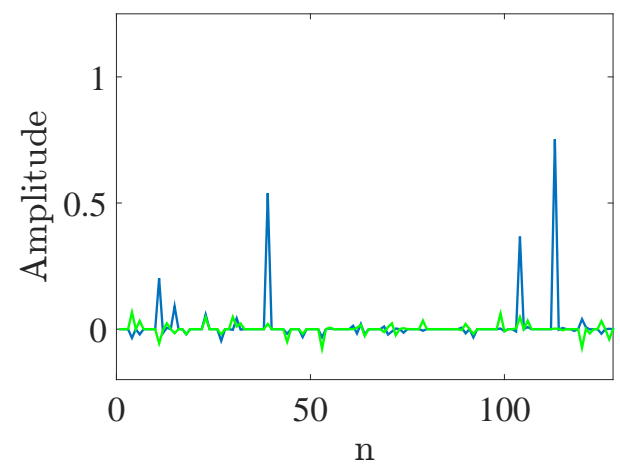

(c)

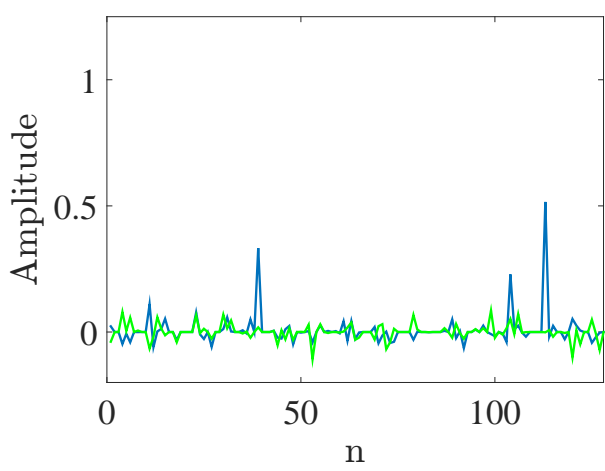

(b)

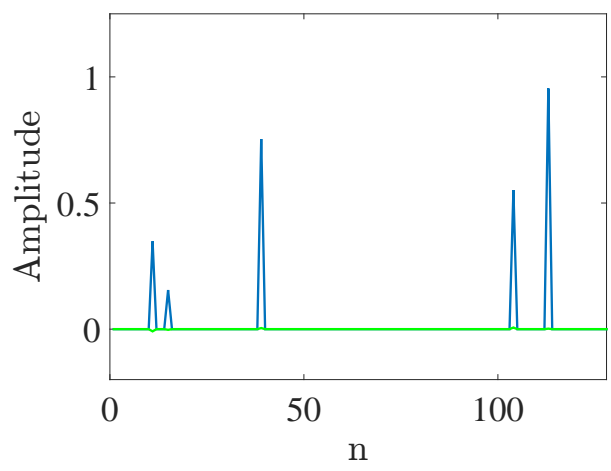

(d)

Figure 3.4: The iteration of reconstruction procedure after (a) 1, (b) 15, (c) 35 and (d) 100 steps. The blue and green bars represent the real and imaginary parts, respectively. 
rewritten as

$$
\operatorname{argmin}\left\|\Phi \mathscr{F}\left(\mathbf{K}_{\mathbf{u}}\right)\right\|_{1}, \quad \text { subject to }\left\|\mathbf{I}_{\mathrm{e}}-\mathscr{F}\left(\mathbf{K}_{\mathrm{u}}\right)\right\|_{2}<\epsilon,
$$

where $\mathbf{K}_{\mathrm{u}}$ and $\mathbf{I}_{\mathrm{e}}$ are the undersampled $k$-space data and the estimated MR image, respectively. $\mathscr{F}$ is the FT operator.

The previous example of the MR image (Figure 2.4) is used to illustrate the procedure of CS-MRI. The $k$-space data will be undersampled in a certain pattern, which is operated by a "mask". Based on the 2D spatialencoding pulse sequence on Section 2.2, it is easier to undersample the $k$-space lines along the phase-encoded direction. The "grating-like" mask is shown in Figure 3.5 (a). The white slabs represent the sampling area in the $k$-space data and the black slabs indicate the corresponding areas in $k$-space which will be un-sampled. The size of this mask matrix is identical to the $k$-space data given in Figure $2.4(\mathrm{~b})$, which is $256 \times 256$. The sampling rate in Figure 3.5 is 0.2 , meaning that only $51(=256 \times 0.2)$ lines in the phase direction are used for future processing. Since the centre of $k$-space covers the contrast of the image, 15 lines in the central area are fully kept. Moreover, the remaining 36 lines are not evenly but randomly spaced $[89,90]$. When operating this mask matrix with the original $k$-space data, an undersampled $k$-space can be simulated as shown in Figure 3.5 (b). The intensities in $k$-space are logarithmic in order to increase the contrast of sampled and un-sampled parts.

The reconstructed image can be obtained by applying FT on the undersampled data, which is shown in Figure 3.6 (b). Comparing it with the original image in Figure 3.6 (a), no aliasing but "incoherent" artefacts are seen along the horizontal axis, which denotes phase-encoded direction in $k$-space (Figure 3.5 (a)). The difference (error image) between this image and the original one is given in Figure 3.6 (c). Key features of this brain image are either missing or blurred along the undersampled axis.

The next step is to find a transform through which the image appears sparse. As discussed in the 1D case, FT is an option. In the meantime, it 


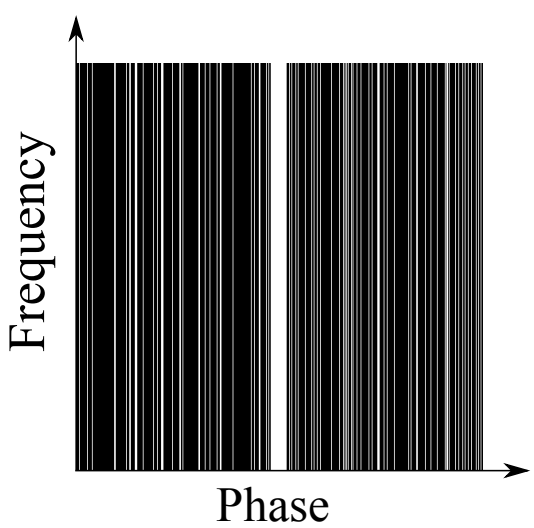

(a)

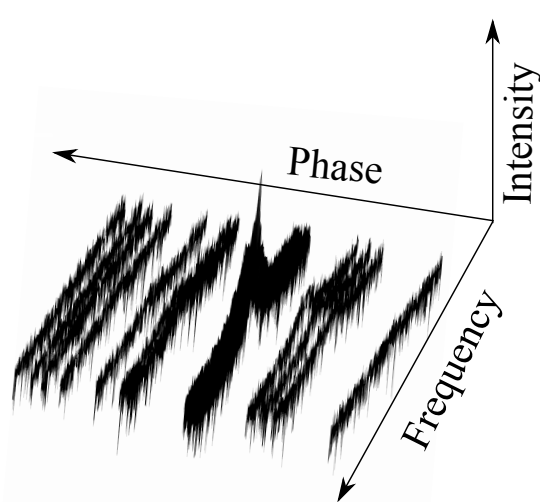

(b)

Figure 3.5: The undersampling mask along the phase encoding direction (a) and operated $k$-space data (b). The intensities in $k$-space are logarithmic in order to enhance the contrast of sampled and un-sampled parts.

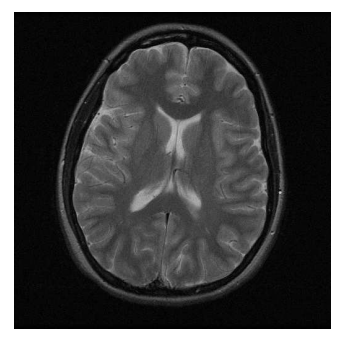

(a)

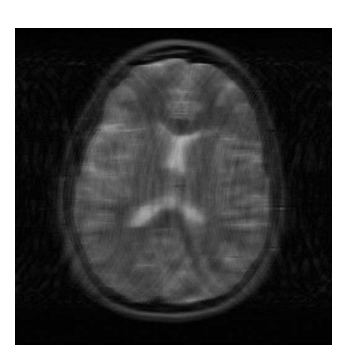

(b)

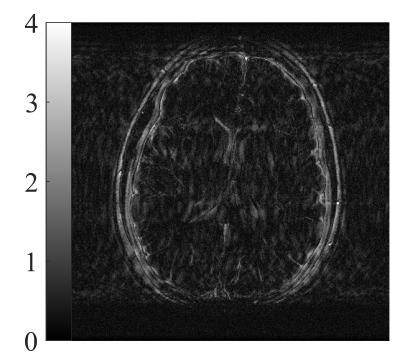

(c)

Figure 3.6: The fully sampled (a), undersampled (b) and the error (c) images using the zero-FT technique. 
is evident that wavelet transform is well accepted in compressing JPEG2000 images $[96,97]$. Unlike Fourier coefficients which only carry either frequency or time message, the wavelet coefficients contain both [98]. These wavelets translate a multi-scale representation of the image. The decoded images by one type of wavelets are pictured in Figure 3.7. Coarse-scale wavelet coefficients represent the low resolution image components and fine-scale coefficients stand for high-resolution components. Therefore, in the $2 \mathrm{D}$ case, wavelet is used as the sparse transform ${ }^{1}$.

A soft threshold value ( $\sigma=0.01$ ) on the wavelet coefficients is used to solve the optimisation equation in Equation (3.3). The reconstructed image is given in Figure 3.8 (a). It is possible to observe the boundary of the brain and some details of in the centre of Figure 3.8 (a) with a quarter of scan time as is required to obtain Figure 2.4 (a). Furthermore, the residual error from the reconstructed image is shown in Figure 3.8 (b). It is obvious that although some high-resolution components still exist in the error image, the residual error is greatly decreased and the central part has been recovered.
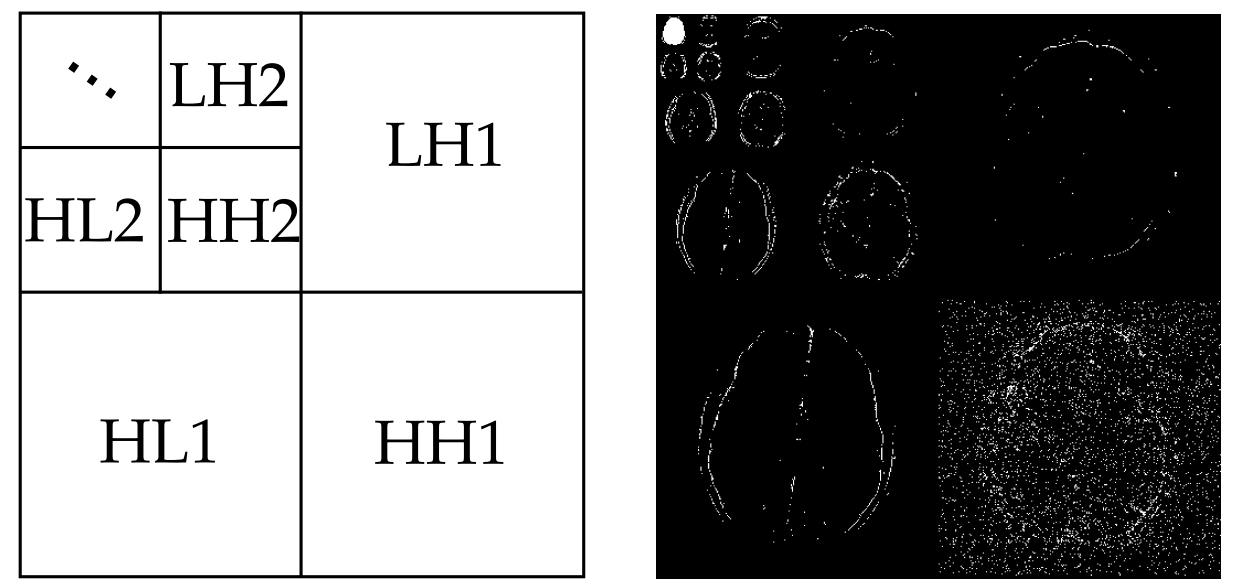

Figure 3.7: Scheme of levels of wavelet decomposition (a) and sparse representation of the brain image in the wavelet domain (b). $\mathrm{H}$ and $\mathrm{L}$ stand for high and low coefficients, and the number afterwards represents the level of decomposition.

\footnotetext{
${ }^{1}$ The detailed implementation of the wavelet-CS algorithm can be found in http: //www.eecs.berkeley.edu/ mlustig/Software.html.
} 


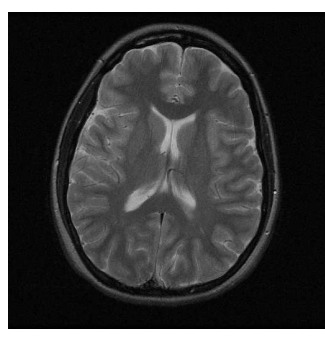

(a)

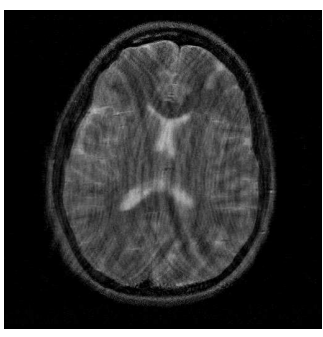

(b)

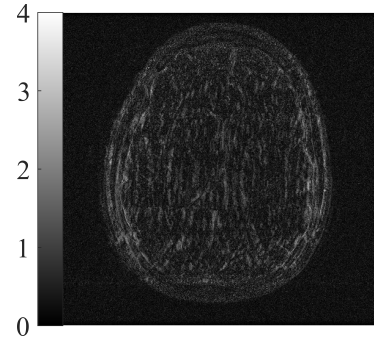

(c)

Figure 3.8: The fully sampled (a), reconstructed (b) and error (c) images using the wavelet-CS technique. The soft threshold value $\sigma$ is 0.01 .

Although this result is visualised better than Figure 3.6, some artefacts can still be observed along the horizontal axis in Figure 3.8 when using wavelet-CS algorithm. This is a known situation in wavelet CS-MRI that the sampling rates are required to be not lower than $30 \%$ to obtain a desired outcome [35]. A data-adaptive sparse domain (such as principal component basis) $[99,100]$ may address this issue which will be discussed in Chapter 4 .

\subsection{Weighted Imaging Techniques}

Imaging contrast in conventional MRI mainly depends on the following parameters: proton density $(\rho)$, longitudinal relaxation time $\left(T_{1}\right)$, transverse relaxation time $\left(T_{2}\right)$ and molecular diffusion coefficients $(D)$. Proton densityweighted images are usually obtained by using the SE imaging sequence (shown in Figure $2.5(\mathrm{a})$ ) with short echo time $\left(T_{\mathrm{E}}\right)$ and long repetition time $\left(T_{\mathrm{R}}\right)$. Thus, signal intensity is a function of the amount of protons in the voxel [101].

$T_{1}$ contrasts is generally obtained using the same sequence but with short $T_{\mathrm{E}}$ and short $T_{\mathrm{R}}$, in which way, proton with high $T_{1}$ value will not have completely recovered and have a reduced intensity than protons with low $T_{1}$ values in the obtained MR Images [102]. For instance, in the cystic astrocytoma, proton in bound water will have lower $T_{1}$ value (because of 
the fast energy exchange) which will exhibit higher intensity to the image.

$T_{2}$ contrast is typically obtained with long $T_{\mathrm{E}}$ and long $T_{\mathrm{R}}$, as a result, proton with higher $T_{2}$ value will dephase slower and appear hyperintense in the image [102]. Taking protons in the necrotic tissue as an example, it has longer $T_{2}$ than that in the normal tissue which will be displayed brighter in the image.

Diffusion contrast is produced by an imaging method which is usually a series of images by applying additional diffusion-sensitising gradients with linearly incremental strength $[103,104]$. Molecules with smaller mobility will provide higher signal thus much brighter than molecules with larger mobility. Moreover, with the increase of the diffusion-sensitising gradients, the image contrast can be enhanced, which will be addressed in Section 3.2.2.

In general, images with these contrast mechanisms are known as weighted images and the corresponding techniques are named as weighted imaging, such as $T_{1}$-weighted imaging (T1WI), $T_{2}$-weighted imaging (T2WI) and diffusion-weighted imaging (DWI). By obtaining a series of specific weighted images, the parameter $\left(\rho, T_{1}, T_{2}\right.$ or $\left.D\right)$ in each pixel can be calculated, thus forming a parametric map.

\subsubsection{Dynamic contrast enhanced MRI}

Apart from earlier specified tissue disparities naturally relying on spin dynamics or spin-bearing molecular motions, the administration of gadolinium-based materials (or more universal, contrast agents) enhances the signal of certain part of tissues due to high magnetisabilities of these materials [27]. These agents are uptaken by tumour angiogenesis reducing the $T_{1}$ value of tumours, thus leading to a hyperintensity on $T_{1}$-weighted images. Such dynamic contrast-enhanced (DCE) $T_{1}$-weighted MRI method has emerged as a useful tool to characterise abnormalities and discriminate malignant and benign lesions in tissues like breasts [105, 106].

DCE-MRI involves repeated imaging before and after the injection of the 
contrast agents. Generally speaking, the growth of neoplastic cells needs a "thirst" for blood supply and these cells are more permeable than cells in normal tissue. Therefore, more amount of contrast agents will be brought to the region via the blood stream. By observing the signal intensity curve over time in the region of interest (ROI), information about tissue pathology can be obtained. Three classical kinetic curves are shown in Figure 3.9 [107]. Type I represents a persistent curve, exhibiting a progressive rise after the administration of the agents, often an indication for benign lesions; Type II contains a plateau, describing no further rise of signal intensity after 90 seconds. This type of curve is widespread in benign and malignant lesions. Type III is a typical curve for locating malignant tumours, which is a "washout" curve. A declined tendency is seen after the initial rising in type III. The rapid washout of the contrast agents may be caused by the nutrient metabolism needed for the proliferation of cancerous cells.

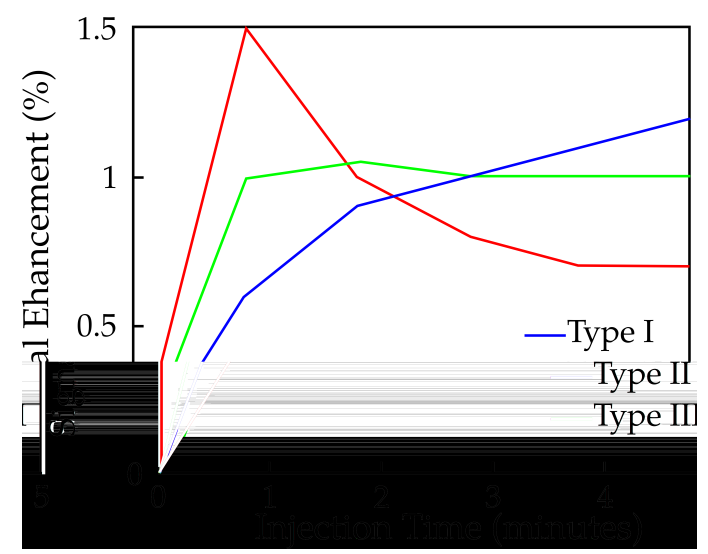

Figure 3.9: The three types of kinetic DCE-MRI curves. Type I: a persistent curve for benign lesions; Type II: a plateau curve for both types of lesions; Type III: a wash-out curve for malignant lesions.

In the recent years, contrast agents have been developed, such as the superparamagnetic iron/iron oxide nanoparticles [108], to improve the diagnosis of small-size tumours due to the enriched $T_{2}$ contrast. Despite the high quality of the images, issues are still remained when using contrast agents in human body $[109,110]$. These particles might penetrate the tissue 
through an impaired blood and brain barrier, which could interfere with supplementary MR examinations. Moreover, these contrast medium may cause permanent reduction in kidney function when they are injected into patients with existing kidney malfunction. High costs associated with purchasing the materials limit their use in rural hospitals. Hence, it is of high significance to develop contrast agent free NMR methods to obtain sufficient image contrast for tissue distinction.

\subsubsection{Diffusion imaging techniques}

As molecular diffusion is independent of magnetic field strength, the change in local field due to contrast agents will not enhance the diffusion contrasts between tissues. Molecule itself can be treated as an "agent" to produce excellent tissue contrast without the injection of any other substance, which is caused by different mobilities of diffusing molecules in tissues. Therefore, these diffusion measurements provide abundant biological and clinical information about tissue composition, micro-structure, and architectural organisation. The following section will introduce different diffusion models in tissues and how to measure them by using MRI pulse sequences.

\section{Diffusion-weighted imaging}

The fundamental technique for every diffusion imaging method is DWI, and the pulse sequence for acquiring DW images is simply a combination of displacement- and spatial-encoding sequences. Various implementations of both encoded gradients have diversified the DWI pulse sequences. Figure 3.10 and Figure 3.11 show two of them which will be used in Chapter 5 and Chapter 6. Specifically, Figure 3.10 is an intuitive example of merging the PGSE and 2D spatial-encoding pulse sequences which were initially introduced in Chapter 2 into one diagram. Whereas, Figure 3.11 incorporates PGSE with the EPI sequence, which is most commonly used in clinical 
application due to its fast acquisition [111]. In the EPI sequence, multiple echoes of different phase steps are acquired by reversing the frequencyencoding gradient and stepping the phase-encoding gradient in between.

However, simply combining diffusion- and spatial-encoding gradient pulses is not trivial. Cross terms due to the tangling between the two kinds of gradient pulses will contribute to the signal decay [113]. As a simplification, a $b$-factor (or $b$-value) was suggested to include the effects from both diffusion and imaging gradients [104], thus the signal attenuation in a voxel is generally described by

$$
M(b)=M_{0} \exp (-b \mathrm{ADC}),
$$

where $b$ is related to the amplitude, duration and separation of the pulsed gradients [104], and the analytic expressions of $b$ in different pulse sequences have been integrated in the standard MRI consoles. ADC is referred to as the apparent diffusion coefficients which is different from the free diffusion coefficients $D_{0}$ in Page 19. By fitting the acquired NMR signal using Equation (3.4) in each pixel, an ADC map can be obtained. An example of images acquired using the DWI-EPI pulse sequence with different $b$-values is presented in Figure 3.12. As can be seen, with the increase of the $b$-value, the signal intensity of each pixel is decreased. However, different parts of the tissue may have distinct decreased rates, leading to the contrast in the ADC map that is given in Figure 3.12.

The above calculation may reflect the true environment of diffusing molecules in materials or plant tissue. Notwithstanding, for tissues which contain vast majorities of randomly oriented micro-vessels, water molecules following the blood stream may hold artificially increased ADC values. In order to quantify the influence from vessels in a single voxel, an intravoxel incoherent motion (IVIM) model was proposed by LeBihan et.al [114]. It is a bi-exponential model separating the fast decay component caused by 


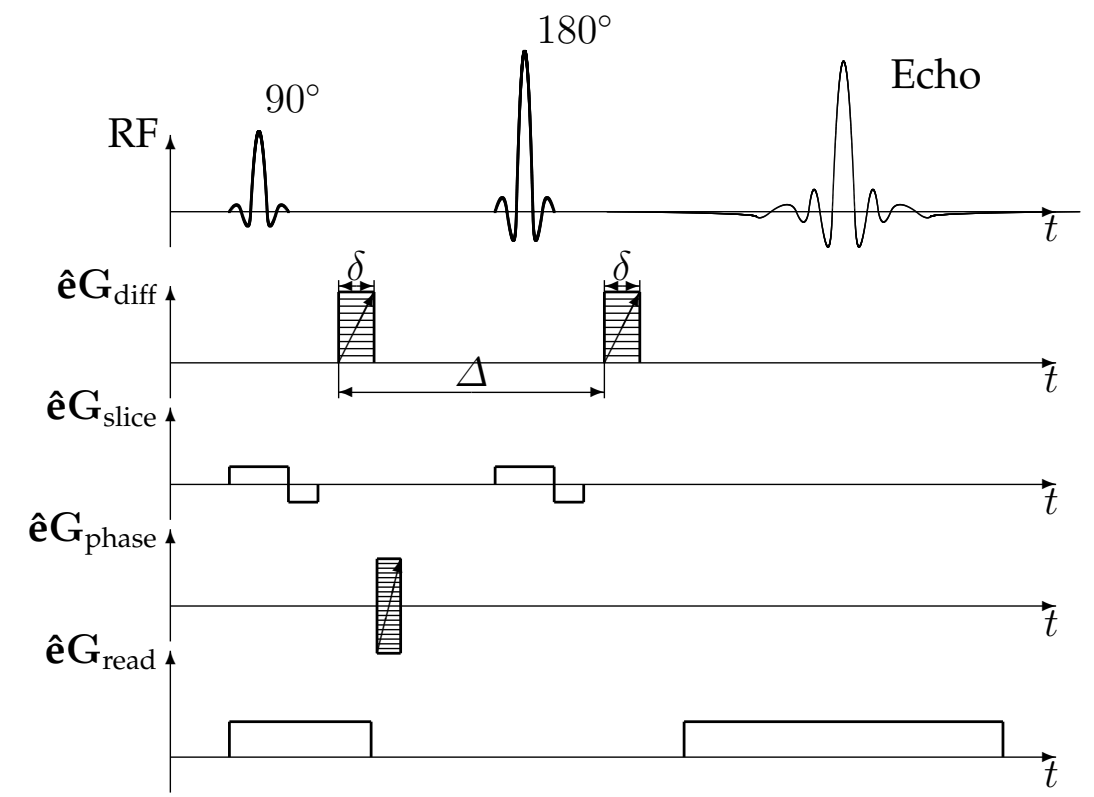

Figure 3.10: Diffusion-weighted spin echo imaging sequence [34].

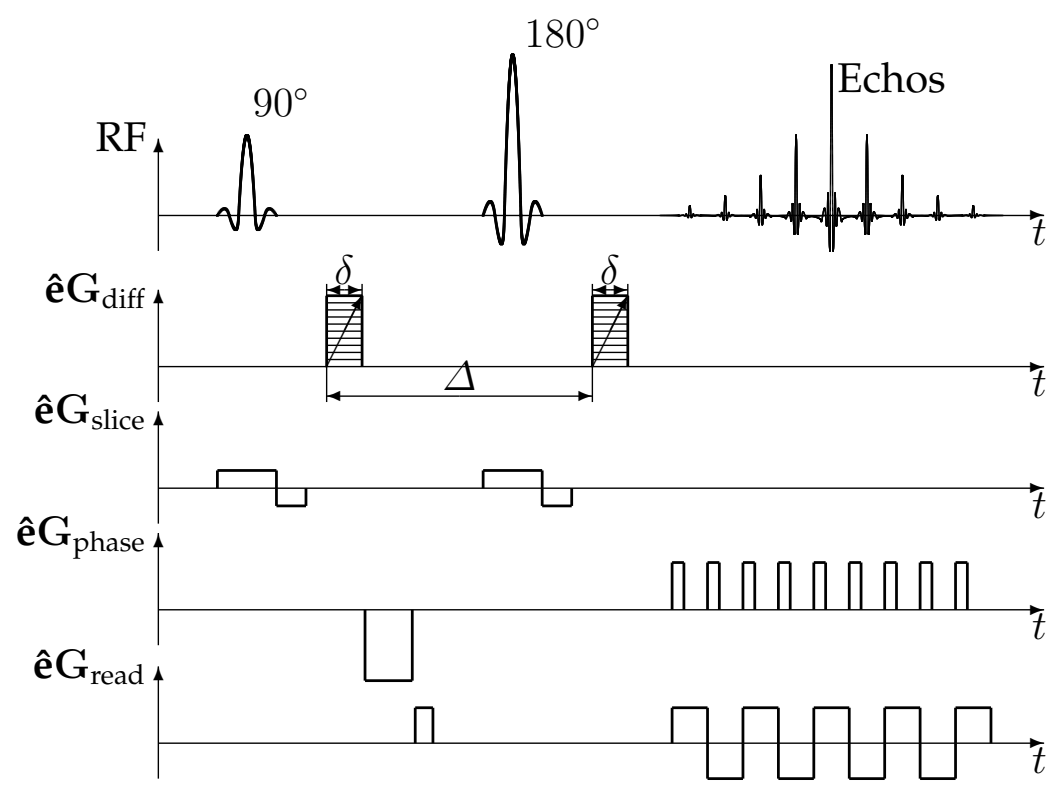

Figure 3.11: Diffusion-weighted spin echo echo-planar imaging sequence [112]. By switching the sign of the frequency-encoding gradient, multiple echoes can be acquired at different phase-encoding gradient strengths. 


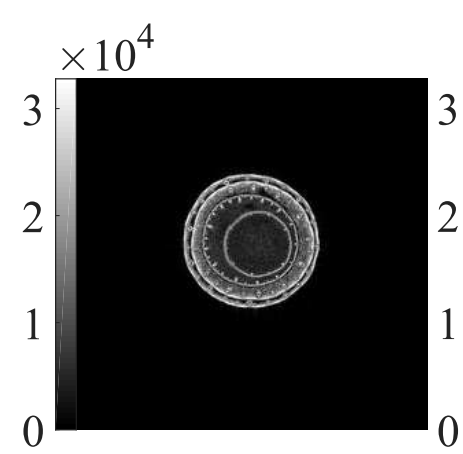

(a)

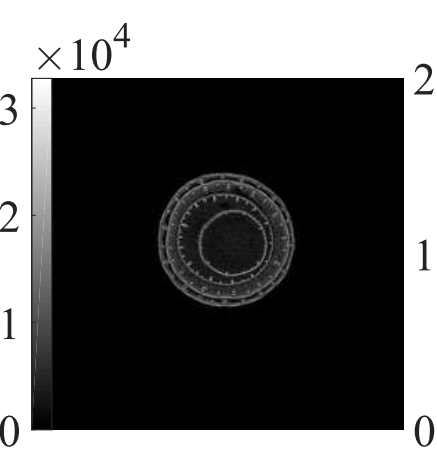

(b)

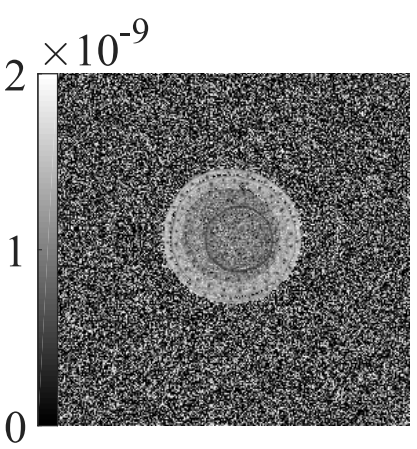

(c)

Figure 3.12: Images of the chive stalk with $b$-values of 0 (a) and $0.5 \times 10^{9} \mathrm{~s} / \mathrm{m}^{2}(\mathrm{~b})$ and its fitted ADC map (c).

the presence of micro-vessels from the self-diffusion contribution [115]:

$$
M(b)=M(0) \cdot f \cdot \exp \left(-b \cdot \mathrm{ADC}_{p}\right)+M(0)(1-f) \exp (-b \cdot \mathrm{ADC}) .
$$

Where, $f$ is the perfusion factor, indicating the fraction of the contribution from the two movements. $\mathrm{ADC}_{p}$ designates the pseudo-apparent diffusion coefficient, which is found sometimes one order greater than ADC [116]. This should be taken into account when fitting the signal using the IVIM model.

\section{Diffusion Tensor Imaging}

So far mentioned NMR diffusometry technique is based on a 1D model of molecular displacement, which is the projection of all displacements onto the axis along which the diffusion gradients are applied. However, molecular diffusion appears in all directions, which is commonly described by a 3D Gaussian model - diffusion tensor (D) [49]. It contains nine elements in 
the laboratory coordinate system:

$$
\mathbf{D}=\left(\begin{array}{ccc}
D_{x x} & D_{x y} & D_{x z} \\
D_{y x} & D_{y y} & D_{y z} \\
D_{z x} & D_{z y} & D_{z z}
\end{array}\right)
$$

whereby the subscripts denote the directions in the Cartesian coordinate system. $D_{x x}, D_{y y}$ and $D_{z z}$ are the diffusion coefficients along $x-, y$ - and $z$-axis, respectively. Whilst $D_{x y}, D_{y x}, D_{x z}, D_{z x}, D_{y z}$ and $D_{z y}$ correspond to the degree of coupling between diffusion in the two indexed directions. Therefore, they can be negative [34]. For electrically uncharged moieties such as water, D is symmetric [34], which means only six uncorrelated elements are necessary to reconstruct the diffusion tensor. The values of these elements are dependent on the spatial structure as well as the orientation of the sample. By rotating this matrix in Equation (3.6) to its principal coordinate system as shown in Equation (3.7), the eigenvalues $\lambda_{1}, \lambda_{2}$ and $\lambda_{3}$ (with $0<\lambda_{1} \leq \lambda_{2} \leq \lambda_{3}$ ) are related to the intrinsic diffusing environment independent of the orientation.

$$
\mathbf{D}=\mathbf{U}^{\mathrm{T}} \boldsymbol{\Lambda} \mathbf{U}=\left(u_{1}, u_{2}, u_{3}\right)^{\mathrm{T}}\left(\begin{array}{ccc}
\lambda_{1} & 0 & 0 \\
0 & \lambda_{2} & 0 \\
0 & 0 & \lambda_{3}
\end{array}\right)\left(u_{1}, u_{2}, u_{3}\right)
$$

where $\Lambda$ holds the eigenvalues and $\mathrm{U}$ includes the eigenvectors. The eigenvectors $\left(u_{1}, u_{2}, u_{3}\right)$ with large eigenvalues indicate the preferred pathways of the diffusing molecules. This means that the motion of the molecules is direction-dependent (i.e. anisotropic). As an opposite, the equivalence of these eigenvalues $\left(\lambda_{1}=\lambda_{2}=\lambda_{3}\right)$ infers an isotropic environment where no preferable direction of molecular diffusion is present.

Figure 3.13 illustrates three diffusion tensor models. In isotropic media, the diffusion tensor can be pictured using a spheric model (Figure 3.13 (a)) where $\lambda_{1}=\lambda_{2}=\lambda_{3}$. However, in anisotropic media, the diffusion tensor 
model is either prolate (Figure $3.13(\mathrm{~b})$ ) where $\lambda_{1}=\lambda_{2}<\lambda_{3}$ or oblate (Figure 3.13 (c)) where $\lambda_{1}<\lambda_{2}=\lambda_{3}$. All three principal directions are coincident with the eigenvectors.
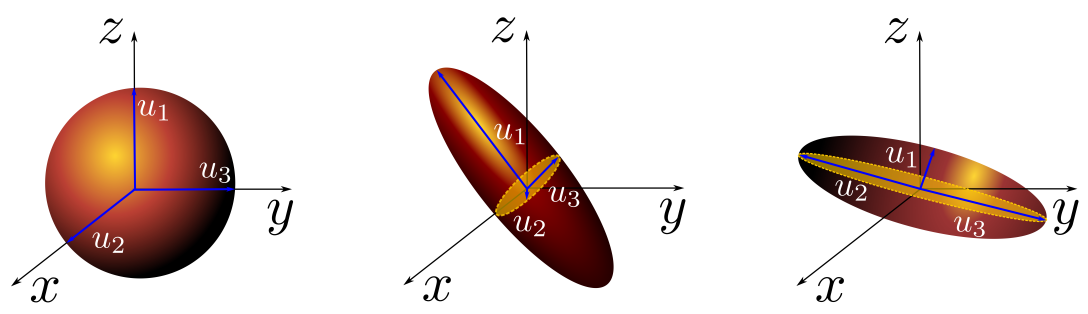

Figure 3.13: Diffusion ellipsoid models: (a) spherical; (b) prolate; (c) oblate.

Diffusion tensor imaging (DTI) is a technique that allows the measurement of the anisotropic diffusion spatially resolved into voxels. It has been used for skeletal muscle, spinal cord, optical nerve and many other investigations, among which, the application of DTI of white matter in the brain is the most prevalent [117-119]. Assuming Gaussian diffusion in each direction, the recorded signal amplitude of the PGSE pulse sequence needs to include the contribution from different directions:

$$
\begin{aligned}
M(b) & =M(0) \exp (-\mathbf{b D}) \\
& =M(0) \exp \left(-\sum_{i=x, y, z} \sum_{j=x, y, z} b_{i j} D_{i j}\right),
\end{aligned}
$$

where $b_{i j}$ is the elements of $\mathbf{b}$-matrix ${ }^{2}$. If the diffusion gradient is applied only along one axis $(x, y$ or $z)$ in the Cartesian coordinates, only single term will contribute to the signal decay:

$$
M(t)=M(0) \exp \left(-b_{i i} D_{i i}\right)
$$

If, however, the diffusion gradient is applied as a vectorised superposition

\footnotetext{
${ }^{2} \mathbf{b}$-matrix is the tensor representation of $b$-value introduced in Section 3.2.2.
} 
of the components from two directions, the PGSE signal intensity is

$$
M(t)=M(0) \exp \left[-\left(b_{i i} D_{i i}+b_{j j} D_{j j}+2 b_{i j} D_{i j}\right)\right](i \neq j)
$$

the coupling of the diagonal and off-diagonal elements in Equation (3.10) make it impossible to extract $D_{i j}$ only relying on single PGSE experiments. Therefore, at least six PGSE experiments are required to be employed in a series with diffusion gradients applied non-collinear, non-coplanar directions to fully reconstruct the elements in diffusion tensor [113].

To evaluate the diffusional anisotropy quantitatively, fractional anisotropy (FA) was defined to give a measure of the asymmetry of the diffusion tensor $[31,120,121]$ :

$$
\mathrm{FA}=\sqrt{\frac{3\left[\left(\lambda_{1}-\lambda\right)^{2}+\left(\lambda_{2}-\lambda\right)^{2}+\left(\lambda_{3}-\lambda\right)^{2}\right]}{2\left(\lambda_{1}^{2}+\lambda_{2}^{2}+\lambda_{3}^{2}\right)}}
$$

where $\lambda$ denotes mean eigenvalue in a pixel, i.e. $\lambda=\operatorname{Tr}(\mathbf{D}) / 3$. Although FA itself is an index which is expressed in the principal coordinate system, Equation (3.11) can still be obtained directly from the non-diagonalised diffusion tensor elements [121]:

$$
\mathrm{FA}=\sqrt{1-\frac{D_{x x} D_{y y}+D_{y y} D_{z z}+D_{x x} D_{z z}-D_{x y}^{2}-D_{y z}^{2}-D_{x z}^{2}}{D_{x x}^{2}+D_{y y}^{2}+D_{z z}^{2}+2 D_{x y}^{2}+2 D_{y z}^{2}+2 D_{x z}^{2}}} .
$$

The relationship of FA $=0$ holds true only when the off-diagonal elements $D_{x y}, D_{x z}$ and $D_{y z}$ are all zero and the diagonal elements $D_{x x}, D_{y y}$ and $D_{z z}$ are identical, which means that molecular diffusion is isotropic; on the other side, FA $>0$ means molecules diffuse anisotropically.

FA value is usually shown as a colour scale in a DTI map to indicate the orientation of fibres. However, the value is an averaged degree of anisotropy in a voxel. Therefore, it is known to be blind to the microstructures [37], which has confounded the diagnostic use of FA in distinguishing the randomly oriented fibres, such as crossing fibres/axons in the 
white matter. In these environments, the FA values will be zero but the micro-structures are not isotropic. Several approaches, such as fractional eccentricity (FE) [122] and microscopical fractional anisotropy $(\mu \mathrm{FA})$ [38], have been suggested recently to overcome this issue. For instance, randomly oriented fibres will return a non-zero $\mu \mathrm{FA}$ value.

It should be noted that FA is a concept built upon the diffusion tensor, however can only be analysed using the DTI measurements up to now. Chapter 5 will introduce an alternative way of obtaining information about FA without imaging parts. Akin to $\mu \mathrm{FA}$, the new approach that is proposed in Chapter 5 can overcome the common crossing fibre issue in FA.

\section{Diffusion Kurtosis Imaging}

The assumption that stands for both DWI and DTI is that the propagator of molecular displacement at each direction obeys Gaussian distribution as shown earlier in Equation (2.11). However, in biological tissues, the presence of barriers (e.g., cell membranes or organelles) and compartments (e.g., intracellular and extracellular spaces) will affect the diffusion behaviour of water molecules. Therefore, the distribution of the propagator may exhibit non-Gaussian features. The non-Gaussianity of the distribution can be quantified by a dimensionless statistic, which is known as kurtosis [123]. A positive kurtosis means the distribution is stronger peaked and heavier tailed as compared to a Gaussian counterpart with the same variance, whilst a negative kurtosis behaviours the opposite way. This can be illustrated in Figure 3.14.

As the diffusion can be generalised as a 3D model, the diffusion kurtosis can be generalised as a tensor accordingly. The diffusion kurtosis is a 4 thrank tensor with 81 components in total [124]. However, the analysis of kurtosis is mainly performed when one gradient direction is applied in this thesis (Chapter 6), therefore, here for simplicity, the 4th-rank tensor is 
reduced to the $1 \mathrm{D}$ case, which is defined by

$$
K=\frac{\left\langle R^{4}\right\rangle_{c}}{\left\langle R^{2}\right\rangle_{c}^{2}}
$$

Gaussian distribution of the displacement probability returns $\left\langle R^{4}\right\rangle=0$ and therefore $K=0$. By substituting Equation (2.17) into Equation (3.13) and rearrange it, the equation becomes

$$
\left\langle R^{4}\right\rangle_{c}=2 D^{2} \Delta^{2} K
$$

Thereafter, Equation (2.16) can be re-written by combining it with Equation (2.17) and Equation (3.14),

$$
M(q)=M_{0}\left[1-4 \pi^{2} q^{2} D \Delta+\frac{(2 \pi)^{4} q^{4} D^{2} \Delta^{2} K}{6}+O\left(q^{6}\right)\right]
$$

Neglecting the higher order terms and substituting $b=4 \pi^{2} q^{2} \Delta^{3}$, a simplified form for signal attenuation can be reached [124]:

$$
M(b)=M(0) \exp \left(-b D+\frac{b^{2} D^{2} K}{6}\right)
$$

Note that $D$ in Equation (3.14), (3.15) and (3.16) represents the Gaussian component of water diffusion in the tissue because it is the variance of the displacement probability distribution.

Figure 3.14 shows examples of three simulated signal attenuations with identical diffusion coefficients but different kurtosis values using Equation (3.16) and its corresponding displacement probability distributions via FT. In diffusion physics, zero kurtosis value indicates a free diffusion behaviour of molecules, whereas positive kurtosis value may be obtained due to restricted diffusion. Negative kurtosis value carries non-physical

\footnotetext{
${ }^{3}$ This is true for PGSE pulse sequence when the diffusion gradient is generated by a narrow pulse.
} 
meaning, however, it can be observed in tissues associated with abnormal phenomena, such as haemorrhage in brain, which leads to the erroneous fit for the acquired NMR data [125].

The acquisition of the diffusion kurtosis is relatively simple, which uses the same protocols with DWI (or DTI) that is available in clinical MRI systems, only with measurements at higher $b$-values ${ }^{4}$ [126]. Subsequently, kurtosis information can be obtained by fitting the signal attenuation using Equation (3.16). This method has been referred to as diffusion kurtosis imaging (DKI) [124].

\subsection{Two-dimensional NMR Spectroscopy}

While MRI techniques locate the position of a spin, MR spectroscopy methods identify the environment of the spin. The environmental information obtained from MRS can be different chemical compositions of the spins in an organic compound (e.g. $\mathrm{CH}_{3} \mathrm{CH}_{2} \mathrm{OH}$ ), or pore size distributions in the porous medium. Two-dimensional NMR (2D NMR) spectroscopy techniques were firstly proposed in 1971 and primarily used in analysing the structure of chemical compounds which cannot be distinguished from 1D NMR spectra [57]. It has the same idea as contained in imaging encoding where both frequency- and phase-encoding gradients are applied to locate the spins in two orthogonal directions. 2D NMR spectroscopy uses two encoding time periods to generate a multiplex signal from direct and indirect domains. Therefore, 2D-FT is applied to process the signal. With the mathematical development of a data processing toolbox, specifically, the inverse Laplace transform (ILT), 2D NMR spectroscopy has been modified and used in a larger scale than the conventional NMR spectrum, to identify fluid types and characterise pore structures, especially where the pore length is beyond MRI resolutions [127-131]. In this section, a 2D NMR spectroscopic method is reviewed, and how it relates to molecular diffusion

\footnotetext{
${ }^{4}$ The $b$-values for kurtosis measurements have been suggested higher than $1 \times 10^{9} \mathrm{~s} / \mathrm{m}^{2}$.
} 

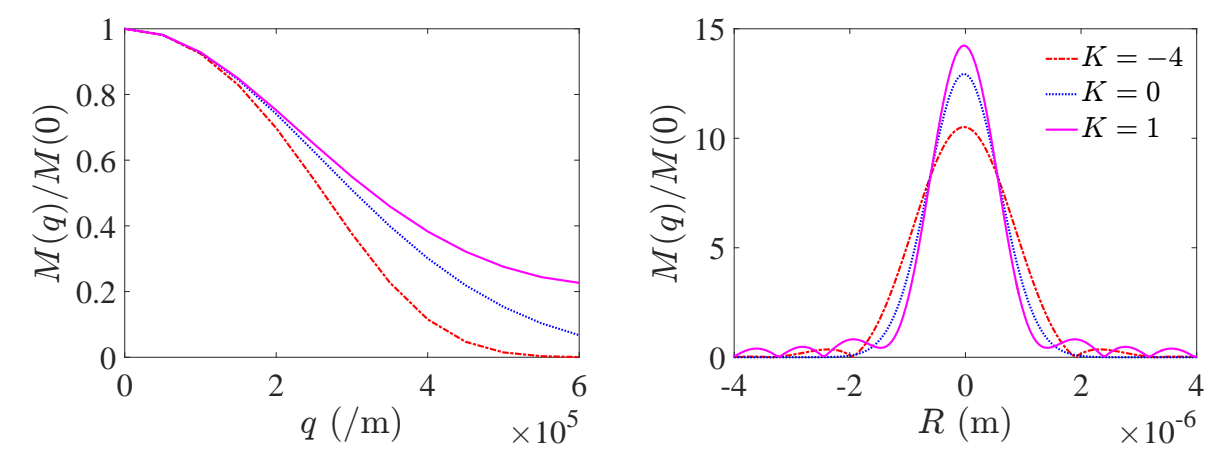

Figure 3.14: Signal attenuation curves (a) and displacement propagator distributions (b) at three different kurtosis values.

will be discussed in Chapter 5 .

\subsubsection{Diffusion-diffusion correlation spectroscopy}

Diffusion-Diffusion COrrelation SpectroscopY (DDCOSY) is a 2D NMR method that allows the observation of local structures without imaging gradients [132-134]. It was proposed with the aim to reveal microscopically anisotropic structures in macroscopically isotropic polydomain systems [135]. In this situation, 1D measurements (single PGSE experiments) return the same signal decay regardless of which direction the diffusion gradient is applied. By appending another diffusion gradients to the single PGSE with a different direction, the obtained signal of this double PGSE [132, 136-142] contains the information of molecular displacement interacting with gradients in two dimensions, holding the signature of local diffusional anisotropy. The pulse sequence is shown in Figure 3.15 and the NMR intensity in DDCOSY is a function of the two wave-vectors $q_{1}$ and $\mathrm{q}_{2}$ [143]:

$$
M\left(\mathbf{q}_{1}, \mathbf{q}_{2}\right)=\int f\left(\mathbf{D}_{\mathbf{1}}, \mathbf{D}_{\mathbf{2}}\right) \exp \left(-\mathbf{q}_{1}^{\mathrm{T}} \mathbf{D}_{\mathbf{1}} \mathbf{q}_{1} \Delta_{1}-\mathbf{q}_{2}^{\mathrm{T}} \mathbf{D}_{\mathbf{2}} \mathbf{q}_{2} \Delta_{2}\right) \mathrm{d} \mathbf{D}_{\mathbf{1}} \mathrm{d} \mathbf{D}_{\mathbf{2}},
$$


The integral in Equation (3.17) indicates that the diffusion coefficients in the sample is a distribution rather than a fixed value. $\mathbf{D}_{1}$ and $\mathbf{D}_{2}$ are the diffusion tensors interacted with the diffusion gradients in the two time intervals ${ }^{5} . f\left(\mathbf{D}_{\mathbf{1}}, \mathbf{D}_{\mathbf{2}}\right)$ is a $2 \mathrm{D}$ distribution function holding the probability of the joint occurrence of $\mathbf{D}_{1}$ and $\mathbf{D}_{2}$. Similar to Equation (3.8), Equation (3.17) can be simplified as by introducing the apparent diffusion coefficients ${ }^{6}$ $D_{1,2}^{a p p}$ :

$$
M\left(q_{1}, q_{2}\right)=\int f\left(D_{1}^{a p p}, D_{2}^{a p p}\right) \exp \left(-q_{1}^{2} D_{1}^{a p p} \Delta_{1}-q_{2}^{2} D_{2}^{a p p} \Delta_{2}\right) \mathrm{d} D_{1} \mathrm{~d} D_{2},
$$

Equation (3.18) shows a Laplace transform from $f$ to $M$, and it is a classically ill-posed problem to obtain from $M$ to $f$. A 2D-ILT was introduced by Venkataramanan and Song $[144,145]$ to solve this problem through an optimisation approach [146, 147]:

$$
\hat{f}=\underset{f \geqslant 0}{\operatorname{argmin}}\left\|M-K_{1} f K_{2}\right\|^{2}+\varrho\|f\|^{2}
$$

where $\|\cdot\|$ is the Frobenius norm of the matrix and $K_{1}$ and $K_{2}$ are the kernel functions constituted from the exponential factors in Eq. (3.18). $\varrho$ is the smoothing parameter controlling the stability in the estimated distribution. This data processing protocol has been successfully applied in the study of porous materials [129, 132-134, 148, 149]. The stability of this protocol was investigated in detail $[130,144,145]$ and uncertainties of the algorithm are discussed in the literature [150-154].

After processing the acquired signal decay by using this 2D-ILT protocol, $f\left(D_{1}^{a p p}, D_{2}^{a p p}\right)$ can be displayed in a correlation map referred to as a $D$ - $D$ map. Diffusivities along the main laboratory axes will be labelled $D_{i i}$ with $i=x, y, z$, e.g. diffusion along the $z$-axis will be designated with $D_{z z}$.

\footnotetext{
${ }^{5}$ Given that the sample doesn't change dramatically and the observation times in the two encoding time are identical, physically $\mathbf{D}_{\mathbf{1}}=\mathbf{D}_{\mathbf{2}}$. However, the subscripts are still kept in order to differentiate these two encoding periods.

${ }^{6} D^{a p p}$ is a substituted symbol of ADC in the spectroscopic context.
} 

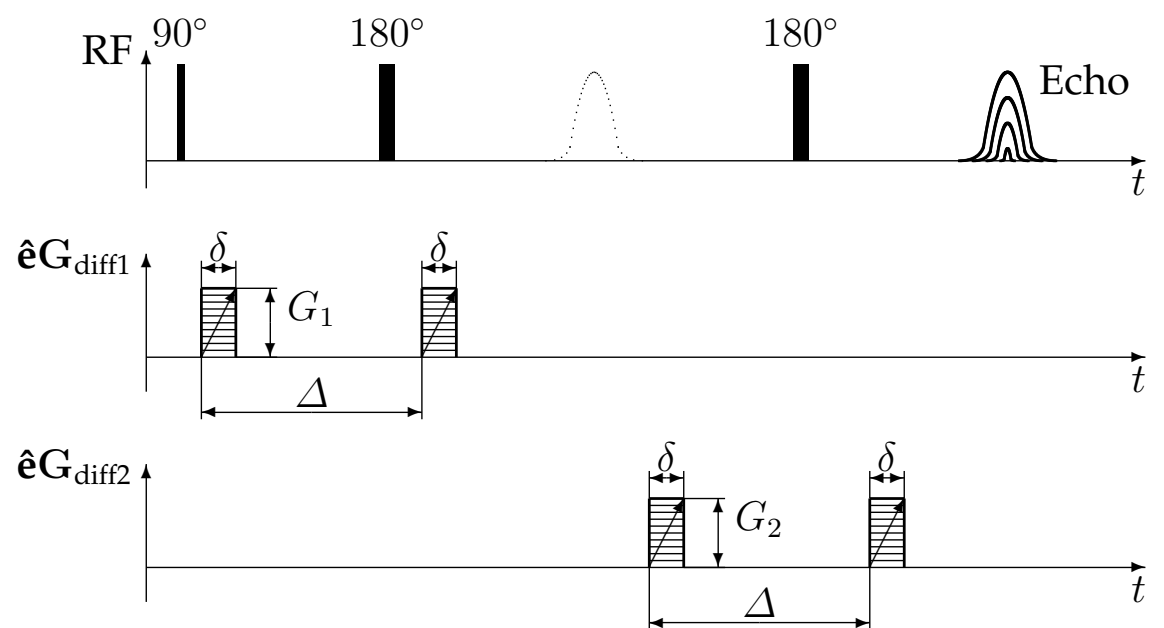

Figure 3.15: The DDCOSY pulse sequence based on Hahn echo. $\mathbf{G}_{\mathrm{diff} 1,2}$ is the pulsed field gradient applied on each direction. The symbol ê in the front represents the unit vector in the direction of the respective gradient in the laboratory coordinate system.

Spatial orientations in the two diffusion domains will be referred to as $D_{i j}$ with $i, j=x, y, z$. This allows for the designation of apparent diffusivities along directions which are linear combinations of the laboratory coordinate system e.g. $D_{x y}$. The 1D distributions of diffusion coefficients along certain spatial directions can be obtained from the 1D projections onto the axes of the $D-D$ map. Isotropic features will manifest themselves through diagonal peaks in the $D-D$ maps while the anisotropic features will appear in off-diagonal peaks. It should be noted here that although negative peak amplitudes are often present in 2D spectroscopic experiments (such as carbon-13 NMR spectra [155] and $T_{1}-T_{2}$ correlation [156]), they are not observed in the DDCOSY experiments due to identical spin dynamics (i.e. transverse magnetisation) involved in both dimensions [66].

Figure 3.16 shows an example of a 2D signal decay acquired using the DDCOSY pulse sequence and the corresponding $D-D$ map in a chopped chive, reproduced from [148]. The diffusion coefficients $D_{y y}$ and $D_{z z}$ plotted on the horizontal and vertical axes represent the diffusion coefficient along $y$ - and $z$-system. The colour bars represent the probability of diffusion 
coefficients at different values in arbitrary units. The upper and right panels are the 1D projections from the 2D map. Diagonal lines in the $D$ $D$ maps mark identical diffusion coefficients in the two dimensions and, thus, isotropic behaviour. Peaks on the diagonal lines will be referred as "diagonal peaks" hereafter, while the "off-diagonal peak" mean these peaks are lying below or above the diagonal lines. In this example, both isotropic and anisotropic diffusion behaviours are observed. The pattern of this map will be used in Chapter 5 .
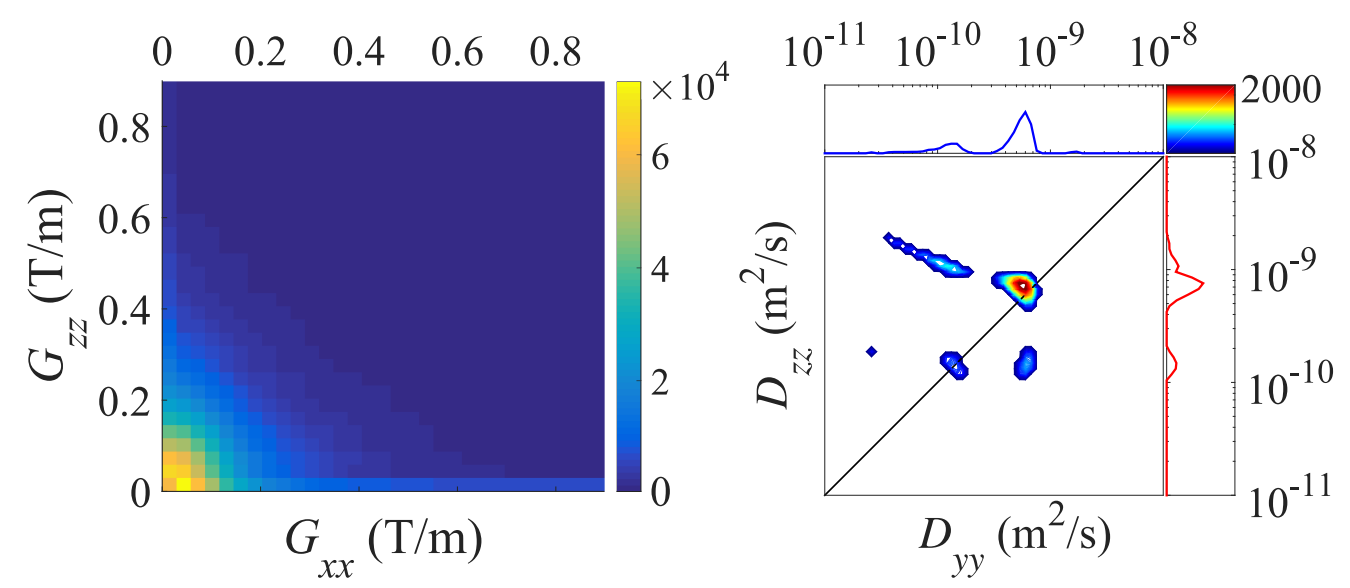

Figure 3.16: 2D DDCOSY signal decay (a) and the corresponding $D-D$ map (b) of chopped chives. The colour bar in (a) indicates the signal intensity. The diffusion coefficients $D_{y y}$ and $D_{z z}$ plotted on the horizontal and vertical axes represent the diffusion coefficient along $y$ - and $z$ - system. The colour bars represent the signal intensity in (a) and the probability of diffusion coefficients in (b) with arbitrary units. The diagonal line indicates the equality of diffusion coefficients along two axes. The upper and right panels are the 1D projections from the 2D map. Reproduced with permission from [148].

As the DDCOSY technique inherently investigates local diffusion anisotropy, it holds similar information that the $\mu \mathrm{FA}$ (mentioned in Section 3.2.2) can provide. This enables the development and modification of the current set-up of DDCOSY experiments and obtain local anisotropy information in the system. 


\subsection{Principal Component Analysis}

Principal component analysis (PCA $)^{7}$, including one-dimensional PCA (1D-PCA) [157] and two-dimensional PCA (2D-PCA) [158], is a statistical technique that analyses a database in which elements are described by inter-correlated variables. The objective of the analysis is to extract the main features (i.e. principal components) from the objects in the database and express the information in a set of new uncorrelated variables (aka orthogonal bases). Hence, each element in the database can be reconstructed by a combination of the orthogonal bases and corresponding weighting factors. This multi-variate technique has been extensively used in face recognition [158, 159].

The major difference between 1D-PCA and 2D-PCA is that each element (e.g. a $2 \mathrm{D}$ image) in the database is required to be re-arranged as a $1 \mathrm{D}$ vector prior to $1 \mathrm{D}-\mathrm{PCA}$ whilst $2 \mathrm{D}-\mathrm{PCA}$ directly process a $2 \mathrm{D}$ matrix. It brings the fact that a domain built from the principal components in 1DPCA holds orthogonality and sparsity, while this is not true in 2D-PCA. Owing to these advantages, scientists started to use 1D-PCA in data and image compression since 1996 [157, 160, 161], and it is now a commonly analytical method in many disciplines which reduces highly dimensional datasets to lower dimensionality [162]. For instance, 1D-PCA facilitates the interpretation in a MR spectroscopy study to separate the contribution of individual chemical constituents to the peaks [163]. At this point, elements in the database are MR spectra. After extracting the principal components in the spectra, metabolite compositions can be classified or identified.

In addition, 1D-PCA has been employed recently to obtain parameter mapping ( $T_{1}$ [164] and $T_{2}$ [165]) or improve temporal resolution in DCEMRI [166, 167] from highly undersampled MRI datasets. These datasets are obtained by simultaneously acquiring spatial information (in $k$-space),

\footnotetext{
${ }^{7}$ In most literatures, PCA only refers to 1D-PCA unless explained elsewhere. However, in this thesis, PCA includes 1D-PCA and 2D-PCA to clarify different usages of the two methods.
} 
NMR relaxation or dynamic information as contained in multiple MR images dependent on some evolution time $t$, thus are commonly referred to as $k-t$ data. In this context, elements in the database are MR images. Through the acquisition of similar MR images along the evolution time $t$, redundancy is introduced into the dataset which enables the undersampling during acquisition and subsequently restore missing information in individual MR images via 1D-PCA, capitalising on the redundancy along $t$. This holds true regardless of the particular sampling scheme employed. For instance in $[166,167]$ a uniform undersampling scheme [168] was used, while in $[165,169], k$-space was sampled randomly (in conjunction with the 1D-PCA as a sparse transform along the $t$-domain). Furthermore, 1DPCA was combined with a model-based algorithm $[164,165]$ to linearise exponential decays of $k-t$ data due to NMR relaxation. Thus, the discussed publications [164-167, 170] are built around the additional information (redundancy) along the evolution time $t$ of the $k$ - $t$ data when reconstructing the individual undersampled MR images. However, an approach which does not rely on the existence of the evolution time domain $t$ uses parallel acquisition combined with uniform undersampling [171, 172].

The following section illustrates how to obtain the principal components by using 1D-PCA. Afterwards, this concept will be extended to 2D which will provide the methodological background for Chapter 4 .

\subsubsection{The procedure of 1D-PCA}

The illustration in Figure 3.17 shows how the principal component basis is obtained via 1D-PCA. Before performing 1D-PCA, each of the $d$ images in the database $\left(\mathbf{I}_{1}, \mathbf{I}_{2}, \ldots, \mathbf{I}_{d}\right)$ is re-arranged into a vector $\left(\vec{I}_{1}, \vec{I}_{2}, \ldots, \vec{I}_{d}\right)$, thus the database can be treated as a $L \times d$ matrix $\left(\mathbf{D}_{\mathrm{B}}\right)$. Subsequently, a covariance matrix $\mathrm{C}$ is constructed using the database matrices:

$$
\mathbf{C}=\left(\mathbf{D}_{\mathrm{B}}-\mathbf{M}_{\mathrm{B}}\right)^{T}\left(\mathbf{D}_{\mathrm{B}}-\mathbf{M}_{\mathrm{B}}\right) .
$$


$\mathbf{M}_{\mathrm{B}}$ is a $L \times d$ matrix where the columns are identical and equal to the vectorised mean image:

$$
\vec{I}_{\mathrm{m}}=\frac{1}{d} \sum_{n=1}^{d} \vec{I}_{n} .
$$

As a consequence, the elements in the covariance matrix represent the correlation of each pixel among the images in the database. Through the diagonalisation of $\mathbf{C}$, the eigenvectors $(\mathbf{U})$ can be determined and they are ordered according to their corresponding eigenvalues. By projecting the matrix $\left(D_{B}-M_{B}\right)$ on $U$, the principal component matrix $(P C)$ of the database can be obtained by

$$
\mathbf{P C}=\left(\mathbf{D}_{\mathrm{B}}-\mathbf{M}_{\mathrm{B}}\right) \cdot \mathbf{U}
$$

The size of $\mathbf{P C}$ is $L \times d$, in which the column vectors are referred to as the principal components [159] and orthonormal to each other. Individual images in the database can be reconstructed using the principal components together with suitable weighting factors which characterise this image. These weighting factors are denoted as the projection coefficients and constitute a vector PJ in Figure 3.17 which can be determined by projecting this image to the principal component basis.

It is known that PJ may carry negligible weighs, therefore, it may be truncated and the principal components may therefore be used as a sparse domain [100]. The sparsity of this orthonormal basis is evaluated by the ratio of the number of zero elements in $\mathbf{P J}$ to the number of total elements (L).

\subsubsection{The procedure of 2D-PCA}

2D-PCA was proposed by Yang et al. [158] and has been used for feature extraction and data representation. However, it has not been applied to MRI data analysis to date. As introduced earlier in Page 48, 2D-PCA directly processes the $2 \mathrm{D}$ matrices for the extraction of independent features in the 


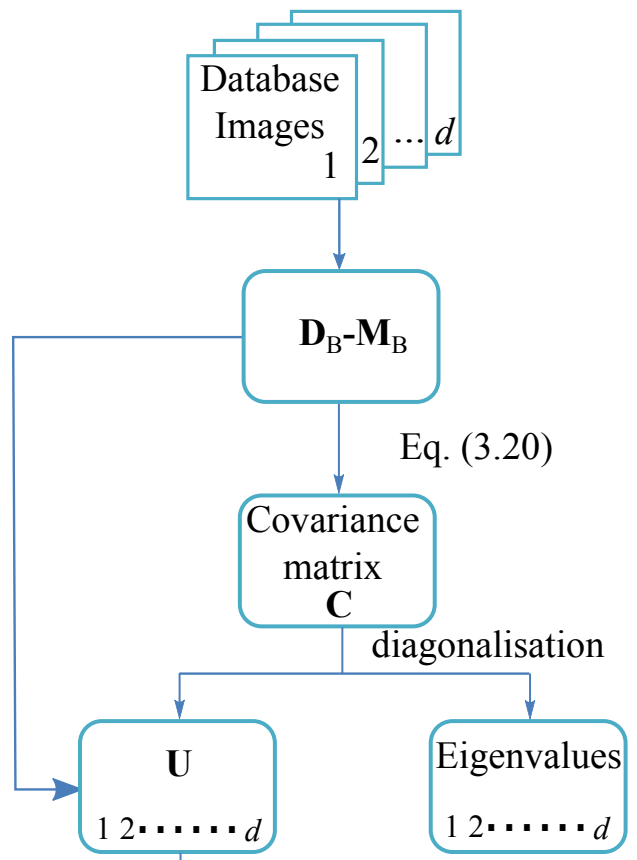

Eq. (3.22)

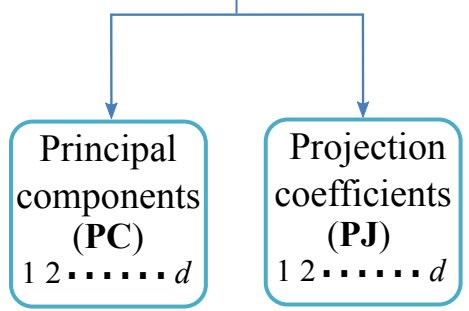

Figure 3.17: Flow chart of the 1D-PCA procedure. $\mathbf{M}_{\mathrm{B}}$ is a $L \times d$ matrix in which the columns are identical, equal to the vectorised mean image; $\mathbf{U}$ is a $d \times d$ matrix containing the eigenvectors after eigen-decomposing the covariance matrix $\mathbf{C}$. PJ are the full set of coefficients. 
image. In 2D-PCA, the image covariance matrix $(\mathbf{C})$ is constructed by using the original $d$ images which are represented by $M \times N$ matrices:

$$
\mathbf{C}=\frac{1}{d} \sum_{i=1}^{d}\left(\mathbf{I}_{i}-\hat{\mathbf{I}}\right)^{T}\left(\mathbf{I}_{i}-\hat{\mathbf{I}}\right)
$$

where $\mathbf{I}_{i}$ is the $i$-th image and $\hat{\mathbf{I}}$ is the mean image matrix. According to Equation (3.23), the size of the covariance matrix $\mathbf{C}$ depends on the size of the column of the image. Therefore, it has a square size of $N$ by $N$. Each element in $\mathbf{C}$ is then the average of the correlation magnitudes between columns in this set of images. By diagonalising $\mathbf{C}$, a matrix of eigenvectors (U) and their corresponding eigenvalues are obtained. These eigenvectors in $\mathbf{U}$ have been proven to be the optimal axis for feature extraction [158]. Through the direct projection of the $2 \mathrm{D}$ image matrix on $\mathrm{U}$, the feature matrix of principal components $\left(\mathbf{P C}^{i}\right)$ of the $i^{\text {th }}$ image in the database can be obtained and expressed by

$$
\mathbf{P C}^{i}=\left(\mathbf{I}_{i}-\mathbf{I}\right) \cdot \mathbf{U}
$$

where $\mathbf{P C}^{i}$ is a $M \times N$ matrix, for which columns are the principal components of the image. After projecting all the images in the database to $\mathbf{U}, \mathrm{a}$ $3 \mathrm{D}$ dataset of principal component matrices $\left(\mathbf{P C}^{1}, \ldots, \mathbf{P C}^{d}\right)$ can be collected. This procedure of 2D-PCA is illustrated in Figure 3.18.

\subsection{Biological tissues studied in this thesis}

Three different biological tissues were used in this thesis to either prove the feasibility of the proposed methods experimentally or as objects for field study. This section will provide relevant information of these tissues for the discussions in Chapter 4, 5 and 6. 


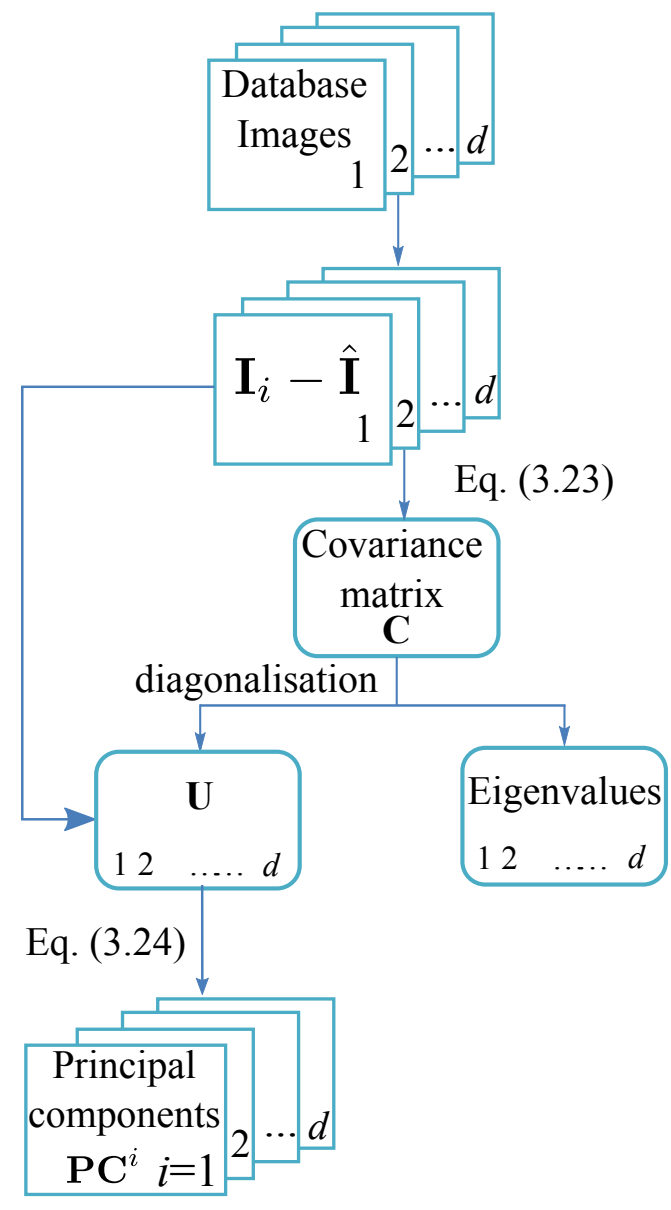

Figure 3.18: Flow chart of the 2D-PCA procedure. $\mathbf{I}_{i}$ and $\hat{\mathbf{I}}$ are the $i$-th and the mean image matrices, respectively. $\mathbf{P C}^{1}, \ldots, \mathbf{P C}^{d}$ are the principal components of the database. 


\subsubsection{Plant tissue - carrot}

As a widely available biological tissues, carrots were used in this thesis to build up an image database in Chapter 4 . A cross section of the carrot tissue is seen in Figure 3.19. From the outer to the inner layer, it is mainly composed of epidermis, cortex, pericycle, xylem and phloem.

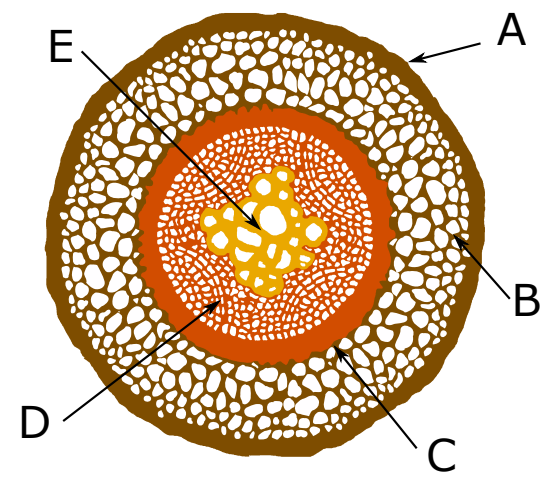

Figure 3.19: The cross section of a carrot.
A - Epidermis;
B - Cortex;
C - Pericycle;
D - Xylem;
E - Phloem

Epidermis is the single exterior layer that protects against water loss and absorbs water from the external environment. The epidermal cells are typically more prolonged than other parts of the tissue, with a radial and azimuthal in-plane diameters of around $30 \mu \mathrm{m}$ and $75 \mu \mathrm{m}$ [173]. The cortex cell, however, has a smaller diameter in the azimuthal direction and slightly larger length in the plane. The most important function of the cortex is to transport the water and nutrients into the central cylinder (which is not shown in Figure 3.19), while xylem conducts water and nutrients from the roots throughout the plant. Xylem tissue is structurally complex, composed of a series of long tubes made up of shorter vessels. The transport function of the cortex and xylem makes the cell wall very thin and permeable. Phloem is the innermost layer of Figure 3.19, transporting sugar from photosynthesis throughout the plant, holding the elongated cells. However, the centre cylinder of the carrot is composed of more rounded cells, with a spherical diameter of around $100 \mu \mathrm{m}$.

The distinct shapes and diameters of different compartments in the carrot make it a sample suitable for the investigation of isotropic and 
anisotropic environments in plant tissues [84,173, 174], as will be presented in Chapter 5.

\subsubsection{Animal tissue - mouse brain}

It is known that a tumour usually indicates an abnormal growth of tissue, therefore, the structure of normal tissues may be changed due to the tumour development. In order to test the capability of a NMR method for identifying these structural changes, two examples of healthy tumourbearing mouse brains were used in Chapter 5 . The morphological information of a healthy adult mouse brain is briefly shown in Figure 3.20 [175-178].

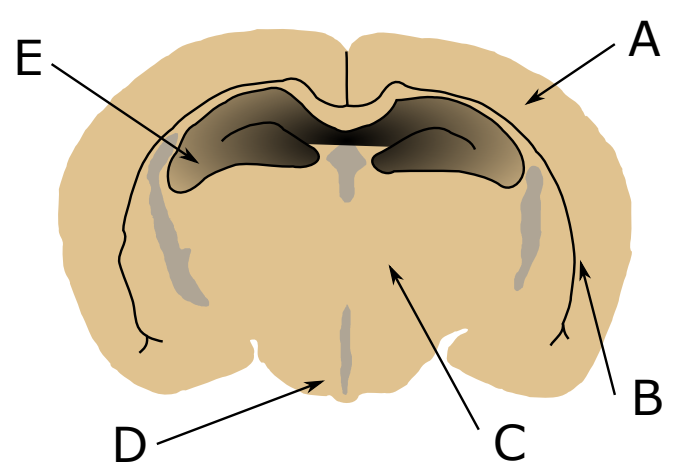

Figure 3.20: Normal mouse brain anatomy.

A - Cerebral cortex;

B - External capsule;

C - Thalamus;

D - Hypothalamus;

E-Hippocampus.

It can be seen that the mouse brain is radial symmetric. Two cerebral hemispheres are clearly divided by the longitudinal fissure, and covered by the cerebral cortex. It is gray matter, consisting mostly of cell bodies and capillaries. The layer below is white matter, which consists mainly of glial cells and myelinated axons. These axons are transmission lines known as nerve fibres. By tracking the orientation of the fibres, it is possible to understand how information is transmitted throughout the neurons, which is usually accomplished by using the DTI technique. Hippocampus reaches the edge of the cerebral cortex, and is shaped as a curved tube in Figure 3.20. The thalamus is located near the centre of the brain, with nerve fibres projecting out of the cerebral cortex in all directions. This leads to the existence of the crossing fibres inside the thalamus. The hypothalamus is a 
small region located below the thalamus, holding complex white matter connectivities.

It should be mentioned here that the human brain has similar anatomic structures as compared to the mouse brain despite larger size and more complex neural networks. As a consequence, the detailed structures of the human brain will not be discussed.

\subsubsection{Human tissue - breast}

Breast tissue was used in a field study contained in this thesis work (Chapter 6). It is supported by the ribs and the pectoral muscles of the chest wall (Figure 3.21). Breast tissue is mainly made of glands (including lobes, ducts) and fat [179].

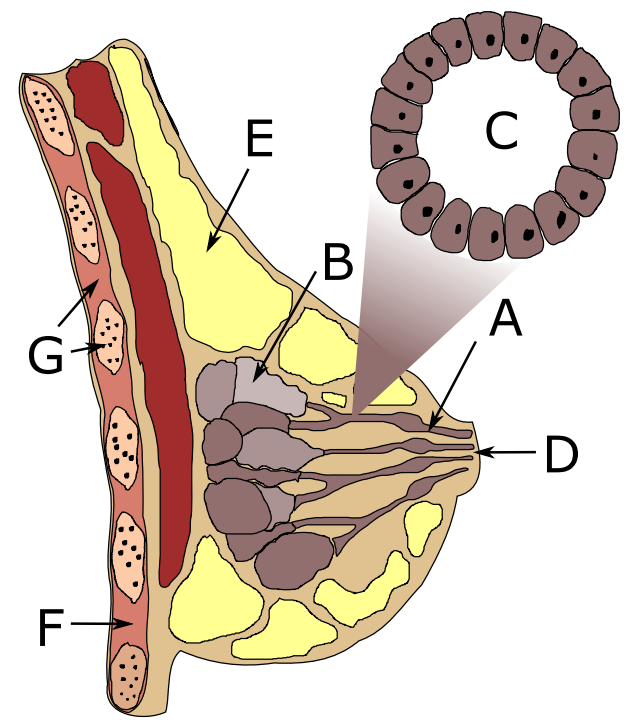

Figure 3.21: Normal breast anatomy.
A - Ducts;
B - Lobules;
C - Cross-section of A;
D - Nipple;
E - Fat;
F - Pectorals major muscle;
G - Chest wall.

\section{Breast lesions}

Breast lesions are abnormal changes in breast tissue due to disease or injury. However, not all lesions are cancerous; only these diseases due to the uncontrollable cell growth and division are known as breast cancers. Cancerous neoplasms can spread to more distant parts of the 
body through the lymphatic system or blood stream. They are usually classified by different histopathologies, grades, stages and the expressions of proteins and genes [107]. The studied lesion types in this thesis are briefly introduced below.

- Invasive ductal carcinoma (IDC): It is one type of malignant tumour that begins in the ducts but invades more into the rest of breast tissues, and it carries the potential of spreading to other organs of the body. IDC is the most common breast cancer, accounting $55 \%$ of the total population of breast cancers.

- Ductal carcinoma in situ (DCIS): It is a non-invasive malignant lesion that is grown within ducts, accounting $13 \%$ of the total breast cancers.

- Invasive lobular carcinoma (ILC): It is an invasive lesion that starts from the milk-producing lobule cells, accounting $5 \%$ of the total breast cancers.

- Cyst: A non-cancerous fluid-filled sac in the breast, usually has a round or oval edge.

- Fibroadenoma: A common non-cancerous solid lesion with a clear edge. It origins from the terminal of the lobules and has high mobility.

- Papilloma: A non-cancerous, nipple-like tumour that arises from the ducts.

Despite of various types of breast cancers, the most common screening method of them is X-ray mammography [110]. It primarily relies on the presence of calcifications, which appear bright on a mammogram. These calcium deposits usually indicate the presence of cancer or other disease. The assessment of the lesion based on X-ray is breast imaging reporting and data system (BIRADS) [180]. For instance, BIRADS 0-2 indicates benign or no suspicious findings; BIRADS 4-5 means that there is a mammographic 
appearance in the image which is suspicious or highly suggestive for malignancy.

For an x-ray image of the breast to be acquired, the breast is compressed to reduce overlap of tissue and decrease the scatter of photons. This, however, represents a major source of discomfort to patients. Furthermore, mammography makes use of ionising radiation, which is harmful to the imaged breast tissue. In contrast, MRI has gained its acceptance in breast imaging as it is non-invasive. The issue of current state of MRI in detecting breast lesions and the contribution of this thesis to this field will be discussed in Chapter 6. 


\section{Chapter 4}

\section{Tissue Identification by Fast Reconstruction of Highly Undersampled MRI Data}

Imaging of tissues is often time-consuming. Recent CS techniques allow signal acquisition with fewer sampling points than required by the Nyquist-Shannon theorem. However, prior knowledge becomes essential to reconstruct detailed features of the imaged tissue when the sampling rate is exceedingly low. As the beginning of the original work presented in this thesis, this chapter introduces fast MRI algorithms to obtain tissue features. A CS scheme developed in wireless sensing networks is adapted for the purpose of reconstructing magnetic resonance images. Moreover, other related reconstruction methods are proposed based on the idea of fingerprinting. These algorithms are demonstrated to be feasible and efficient at high undersampling rates after the comparison with wavelet-CS. This enables the location of some features that are abnormal in tissues more quickly. 


\subsection{Introduction}

CS-MRI permits sampling fewer points in $k$-space as required by the Nyquist-Shannon theorem. However, detailed features may not be appropriately reconstructed by using the wavelet basis when the $k$-space is largely undersampled as discussed in Page 31. In addition, the computational time for data processing using a workstation with a $2.4 \mathrm{GHz}$ Intel Xeon processor and 12 GB memory may be in the order of days [36]. In this situation, to reconstruct the undersampled $k$-space data appropriately and rapidly, prior knowledge (obtained from similar images) regarding global (e.g. shapes) or local information (e.g. relative contrast to adjacent features) may become essential for image reconstruction.

As provided in Section 3.4, after reshaping individual datasets into vectors, the principal components extracted from 1D-PCA are suitable to represent the data in a lower dimension [157, 159, 161, 162]. Hence, each dataset in the database can be reconstructed by a combination of the principal components and their corresponding projection coefficients. For objects which are not in the database but are sufficiently similar, they may be approximated by a suitable set of principal components. As this orthonormal basis is sparse, 1D-PCA can be used as a transform domain in CS for reconstruction. This method was successfully implemented and has been proven effective for the recovery of randomly undersampled signals in wireless sensor networks (WSN) [100]. Due to the fact that datasets with various dimensions can be rearranged to $1 \mathrm{D}$ vectors, 1D-PCACS can be adapted to recover undersampled MR images, which will be discussed later on. Although 1D-PCA has been applied for the processing of undersampled MRI $k$ - $t$ datasets $[164-167,169,170]$ or data acquired by multiple coils [171, 172], so-far discussed applications have not addressed the issue of utilising the principal components as a sparse domain inside the framework of CS-MRI.

The procedure proposed in this chapter is based on 1D-PCA but does 
not rely on the existence of $k$ - $t$ data as for [164-167, 169, 170]. Moreover, unlike previous works [171, 172], $k$-space is undersampled randomly using a single RF-coil, thus providing an alternative reconstruction algorithm when parallel acquisition is not available. Our approach is based on a single dedicated PCA database (independent from individual RF coil and receiving channel configurations) providing prior knowledge when reconstructing MR images at very low sampling rates.

The first approach adopts 1D-PCA as a method to generate a sparse transform domain when reconstructing randomly undersampled $k$-space data, which is the core of our 1D-PCA compressed sensing implementation. The second approach is based on a recognition algorithm for reconstructing undersampled $k$ - $t$ data, which is graphically named as magnetic resonance fingerprinting (MRF) [181]. While adapting the concept of fingerprinting into the image direction, 1D-PCA and subsequent 2D-PCA [158] recognition reconstruction algorithms are presented in this chapter.

\subsection{D-PCA Compressed Sensing}

\subsubsection{Methodology}

Independent of the particular sparse domain used, the CS MRI scheme follows a common concept [22]. As discussed in Section 3.1.1, the sparsity of $\Phi$ is crucial for successful recovery of the object image, because it allows a clean representation and efficient compression of the object class. Sparsity basis used in CS can be classified into two categories; pre-defined dictionary and data-adaptative dictionary. The most frequently used methods belong to the pre-defined dictionaries, such as wavelets, discrete cosine and contourlet transform domain [23, 26, 95, 182, 183]. This kind of basis is isolated from the studied image, meaning that the sparsity is largely liable to the individual image. Thus, they can only accurately represent a limited range of images or image features [99]. Furthermore, the sampling rates 
are required to be not lower than thirty percent in order to obtain a desired outcome [35]. The other category is a data-adaptive dictionary, which is a dynamic basis that can be adapted according to the available database of a certain object class [99]. One of the data-adaptive transforms is the principal components obtained via 1D-PCA [162]. This method has been successfully utilised as a sparse transform domain to be employed in CS reconstruction in WSN [100], and will be named as 1D-PCA-CS. In this chapter, the concept of 1D-PCA for CS reconstruction is adapted into MR images.

The purpose of 1D-PCA-CS is to obtain the best possible estimation of the original (fully sampled) image using the undersampled image $\mathbf{I}_{\mathfrak{u}}$ in conjunction with the 1D-PCA algorithm. To this end, an undersampled $k$ space dataset $\mathbf{K}_{\mathbf{u}}$ is prepared. This dataset is created by placing a designed mask on the fully sampled data as explained in Page 29. Thus, the FT of $\mathbf{K}_{\mathbf{u}}$ results in an undersampled image $\mathbf{I}_{\mathbf{u}}$ which will be used in our calculations. By projecting $\mathbf{I}_{\mathfrak{u}}$ onto the principal component basis, a sparse representation (i.e. projection coefficients $\mathbf{P J}^{\prime}$ ) can be achieved. This enables to obtain an approximate image via a suitable subset of principal components even if the sample image is not in the database (but similar enough to the image class constituting the database). According to the $l_{1}$ norm minimisation rules, a proper subset of $\mathbf{P J}^{\prime}$ can be chosen above a threshold value $\delta$ while the components of $\mathbf{P} \mathbf{J}^{\prime}$ which are smaller than $\delta$ will be discarded. This will form a new vector $\mathbf{P} \mathbf{J}^{\prime \prime}$.

In the next step, an image $\mathbf{I}_{\mathrm{PCA}}$ can be reconstructed by this truncated $\mathbf{P J}^{\prime \prime}$ vector and its corresponding $m$ principal components $(m<d)$. The $k$ space data $\mathbf{K}_{\mathrm{PCA}}$ is then obtained using the inverse FT of $\mathbf{I}_{\mathrm{PCA}}$ and the data in the equivalent area in $\mathbf{K}_{\mathrm{PCA}}$ is chosen to replace the initially zero-filled gaps of the undersampled $k$-space data, $\mathbf{K}_{\mathbf{u}}$. By computing the FT of the updated $k$-space, a new undersampled image $\mathbf{I}_{\mathrm{u}}$ is obtained as the input for the next iteration. The reconstruction procedure of this undersampled image will be iterated for $p$ steps until the Euclidean distance of the neighbouring two 
output $\mathbf{I}_{\mathbf{u}}$ is smaller than a pre-set value $\epsilon$ shown in Equation (3.3).

The reconstructed data can be quantitatively evaluated in terms of peaksignal-to-noise ratio (PSNR). PSNR measures the differences between the reconstructed image and the original image, and is defined by [184]

$$
\mathrm{PSNR}=20 \log _{10}\left(\frac{\mathrm{MAX}}{\sqrt{\mathrm{MSE}}}\right)
$$

where, MSE is the mean square error and MAX is the maximum pixel value of the image. PSNR is often used to compare performance of various algorithms.

\subsubsection{Database evaluation}

As a proof of concepts, carrots were chosen to test the feasibility of the proposed algorithm. This thesis work included images from 25 carrot taproots on the 9.4 T Bruker BioSpec pre-clinical MRI system (Figure 2.1), and obtained 200 axial proton density images in total by using the multislice SE pulse sequence. These images were used to construct a database for subsequent algorithm analysis. $T_{\mathrm{R}}$ was $6 \mathrm{~s}$ and $T_{\mathrm{E}}$ was $15 \mathrm{~ms}$. The MR images were sliced with a thickness of $2 \mathrm{~mm}$ and an interval of $4 \mathrm{~mm}$. The field of view was $25 \times 25 \mathrm{~mm}^{2}$ with the resolution of $0.0977 \times 0.0977 \mathrm{~mm}^{2}$. Thus, the size of each slice was $256 \times 256$.

1D-PCA principal components of this database were extracted according to Equation (3.20) and (3.22), for which the first six ones are shown in Figure 4.2 (a) with respect to the descending order of importance to the database. These indices of importance (normalised eigenvalues) of all principal components are shown in Figure 4.2 (b). As can be discovered straightforwardly from the images, the most important feature is the round shape of the carrot (the epidermis tissue, $P C^{1}$ ) as it holds the highest image contrast. The thickness of the epidermis tissue is influenced by the variation of the taproots' size. The second notable feature is the cortex area and the vascular tissue $\left(P C^{2}\right)$, followed by the endodermis area $\left(P C^{3}\right)$. Subsequent 


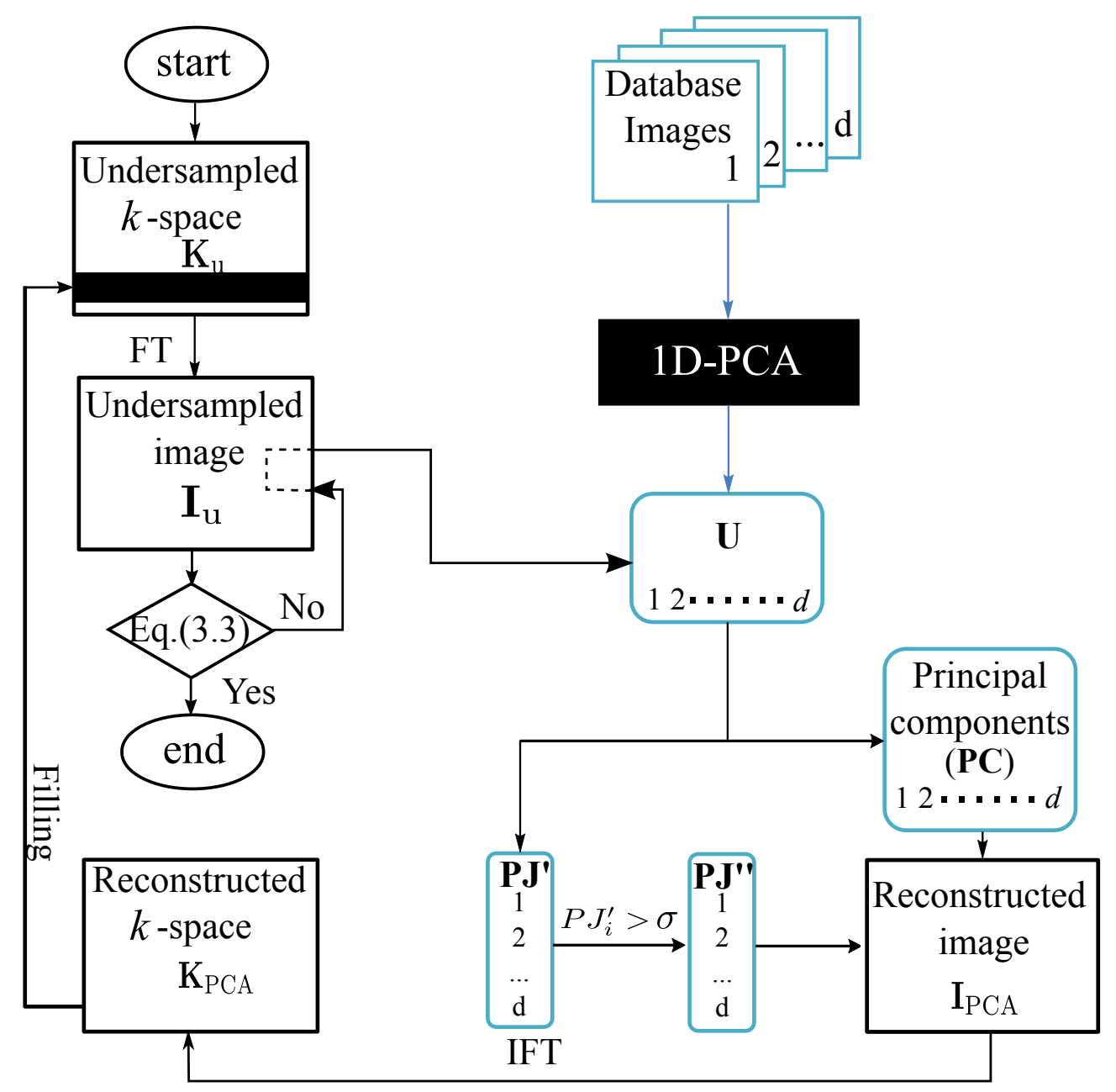

Figure 4.1: Flow chart of 1D-PCA-CS. The black box is the procedure of 1D-PCA, the expansion of which can be found in Figure 3.17. The reconstruction procedure outside the black box starts from randomly undersampling $k$-space (the black lines symbolise the un-sampled areas), and iterated until Equation (3.3) is satisfied. While $\mathbf{P J} \mathbf{J}^{\prime}$ are the full set of coefficients of projecting the undersampled image to the principal components, $\mathbf{P} \mathbf{J}^{\prime \prime}$ represents the truncated set due to the $l_{1}$ and $l_{2}$ minimisation as is given by Equation (3.1). 
high-order principal components characterise more detailed and localised shapes and features.

All images in the database were projected onto the principal component basis, resulting in a map of projection coefficients as shown in Figure 4.2 (c). The order of the principal components is identical to Figure 4.2 (b). The intensities in this map indicate the amplitudes of projection coefficients, representing the weights of the individual principal components. As can be observed in Figure 4.2 (c) the projection values in all images are much larger for low-order principal components, while becoming less significant for high-order ones. Therefore, this basis can be considered sparse enough to be a transform domain for the subsequent CS reconstruction.

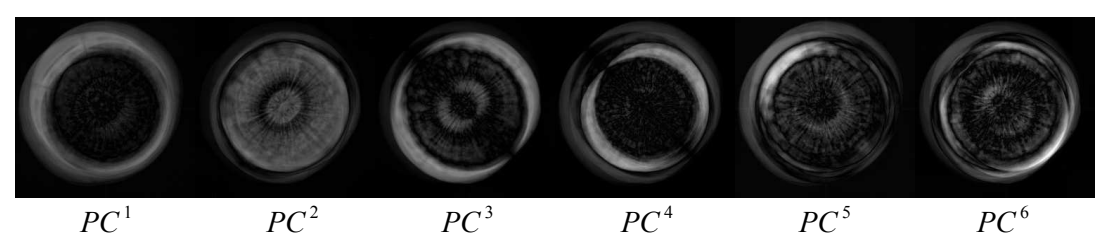

(a)

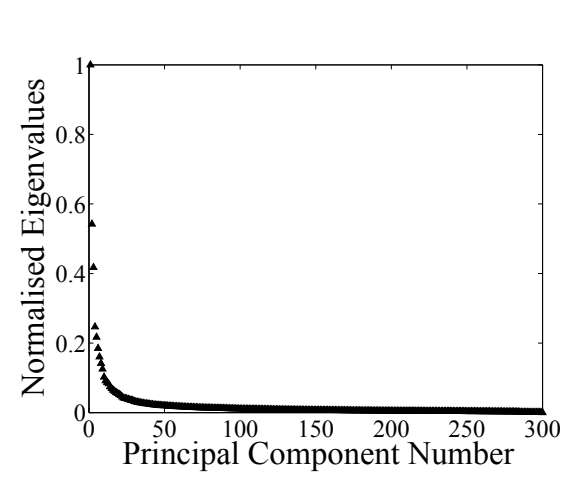

(b)

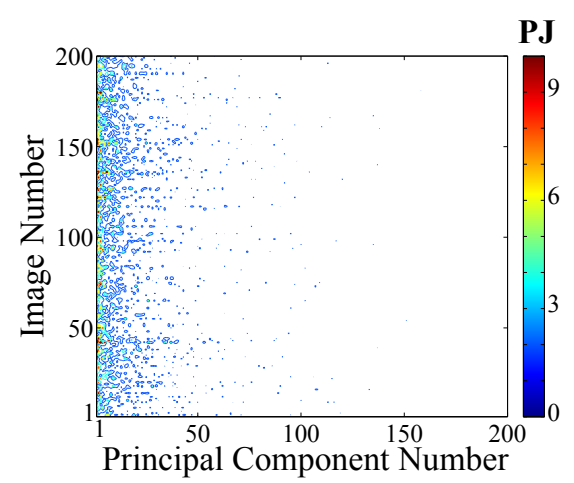

(c)

Figure 4.2: Database evaluation. (a) First six principal components with descending order of importance; b) Eigenvalues of the correlation matrix in Equation (3.20) of each principal component; (c) Map of projection coefficients for all images in the database. 


\subsubsection{Reconstructed results}

The reconstruction results of 1D-PCA-CS are compared using the undersampled $k$-space data in two purposely chosen cases. Case I deals with an image which is included in the database while in case II another image is chosen but not included in the database. Figure 4.3 (a) and (d) are the fully sampled images, where (a) was included in the database (case I) and (d) was excluded from the same database (case II). After applying the undersampling mask to $k$-space as already explained in Page 29, the reconstructed (undersampled) images after FT are shown in Figure 4.3 (b) and (e). Due to high undersampling rate, the aliasing artefacts were significant in the reconstructed images although the random sampling pattern was employed.

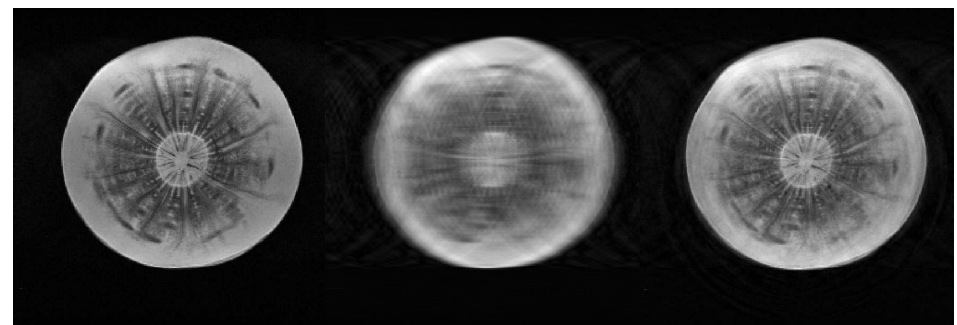

(a)

(b)

(c)

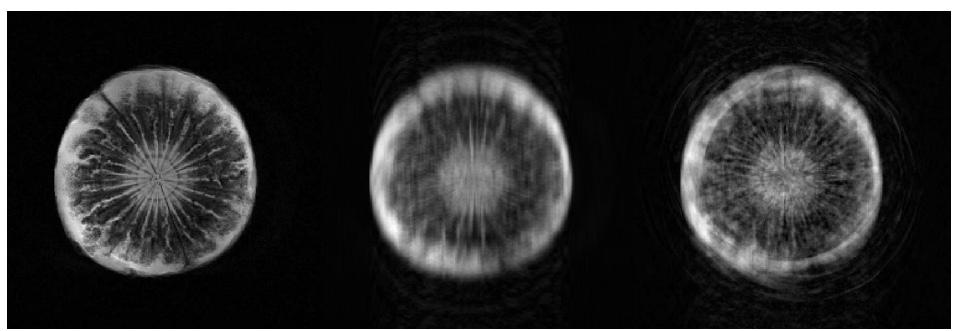

(d)

(e)

(f)

Figure 4.3: 1D-PCA-CS reconstruction results of case I and case II: (a) the full image included in database; (b) the undersampled image in case I (PSNR $=22.5$ ); (c) 1D-PCA-CS reconstructed images in case I (PSNR = 67.3); (d) the full image excluded in database; (e) the undersampled image in case II (PSNR = 19.1); (f) 1D-PCA-CS reconstructed images in case II (PSNR = 21.2). 
In contrast, the aliasing artefacts decreased as shown in Figure 4.3 (c) and (f) when 1D-PCA-CS was applied. The same thresholds $\left(\sigma=10^{-3}\right.$ in Figure 4.1 and $\epsilon=10^{-4}$ in Equation (3.3)) were used in 1D-PCA-CS of both cases, leading to the reconstructed images with a PSNR of 67.3 and 21.2, respectively. The enhancements compared with the undersampled images (i.e. Figure 4.3 (b) and (e)) were 198\% for case I and 11\% for case II. These distinct improvements demonstrated the effectiveness of applying 1D-PCACS to MRI. Not surprisingly, 1D-PCA-CS manifested its superior response when the image was contained in the database. In this case, similar features of the undersampled image and the images in the database were kept in the $l_{1}$ norm minimisation procedure, thus leading to a close approximation of the original image. On the contrary, if features are not part of the database (case II) projection coefficients remain relatively small and will be discarded during the minimization procedure.

\subsection{D-PCA Recognition Reconstruction}

\subsubsection{Methodology}

Although 1D-PCA-CS in case II shows its superiority over the zerofilling FT, the features of the reconstructed results are still unclear. Recently, a novel approach was introduced, namely MRF [181], to overcome these constraints by taking a distinctive post-processing procedure. It uses a dot-product algorithm to match the randomly acquired signal to a predefined dictionary of predicted signal evolutions and select the best match to represent the undersampled data. The inherent merits of 1D-PCA in pattern recognition have been widely strengthened, which allows adapting it in conjunction with the concept of MRF.

Given these merits, 1D-PCA Recognition Reconstruction (1D-PCA-RR) was proposed to improve the image quality. Instead of enforcing only one matched image as a representative, a subset of the MR images are 
chosen to complement the undersampled $k$-space data. The procedure of 1D-PCA-RR is outlined in Figure 4.4. Same with 1D-PCA-CS, a vector of projection coefficients $\left(\mathbf{P J}^{\prime}\right)$ is obtained from projecting the undersampled image to the principal components. Subsequently, a subset of $p$ images in the database are selected if the Euclidean distance $\left(d_{e}^{i}\right)$ between the corresponding $\mathbf{P} \mathbf{J}_{i}$ and $\mathbf{P} \mathbf{J}^{\prime}$ is smaller than $\delta$,

$$
d_{e}^{i}=\left\|\mathbf{P} \mathbf{J}^{\prime}-\mathbf{P} \mathbf{J}_{i}\right\|_{2}<\delta
$$

where $\delta$ is a user-controlling parameter sensitively determining the performance of the algorithm. Once these $p$ images are selected, they are used in the next step to constitute an image $\mathbf{I}_{\mathrm{c}}$ with the corresponding weighting factor, i.e. the inverse of the normalized Euclidean distance:

$$
\mathbf{I}_{\mathrm{c}}=\sum_{i=1}^{p} \frac{1}{d_{e}^{i}} \mathbf{I}_{i}
$$

The image $\mathbf{I}_{c}$ is a weighted combination of the most similar images in the database. By performing the inverse FT of $\mathbf{I}_{\mathrm{c}}$, its $k$-space data $\left(\mathbf{K}_{\mathrm{c}}\right)$ is then used to fill up the gaps of the initially undersampled $k$-space $\left(\mathbf{K}_{\mathrm{u}}\right)$.

Therefore, an updated image $\mathbf{I}_{\mathfrak{u}}$ can be obtained using the FT of the updated $\mathbf{K}_{\mathrm{u}}$ which is now the new input for the 1D-PCA. The protocol will be repeated heading to an iteration until the condition as defined in Equation (3.3) is satisfied. At the end of the iterations, $\mathbf{I}_{\mathfrak{u}}$ will be the best estimate of the original image returned by the 1D-PCA-RR technique.

\subsubsection{Reconstructed results}

The reconstructed results using 1D-PCA-RR of case I and case II are shown in Figure 4.5 with $\delta=10^{-3}$ and $\epsilon=10^{-4}$. To have a better visual comparison, the fully and undersampled (Figure 4.3 (a), (d), (b) and (e)) images of both case I and case II are repeated in Figure 4.5 (a), (d), (b) and (e). PSNR of the reconstructed images using 1D-PCA-RR in case I and 


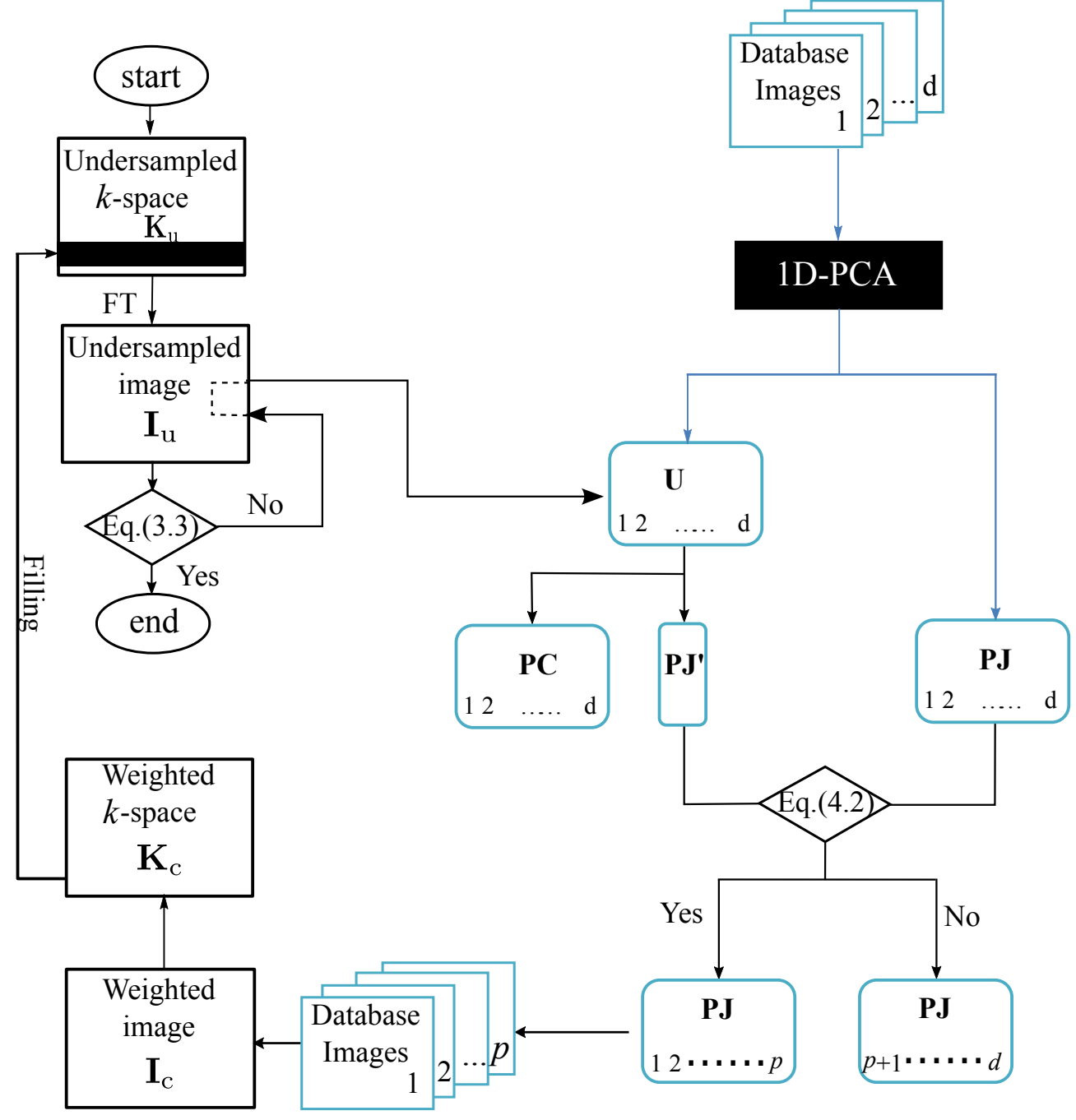

Figure 4.4: The flow chart of the 1D-PCA-RR procedure. The black box is the procedure of 1D-PCA. The procedure outside the black box is iterated until Equation (3.3) is satisfied. PJ are the full set of coefficients of projecting the database image to the principal components, and $\mathbf{P} \mathbf{J}^{\prime}$ is the coefficients of projecting the undersampled image to the principal components. 
case II were 319.6 and 26, respectively, which show improvements of $1318 \%$ and $36 \%$ from their initial inputs (i.e. Figure 4.5 (b) and (e)). As can be seen, case I and case II exhibited surprisingly distinct improvements. This is because in case I, the fully sampled image was in the database, and the algorithm recognised the same image from the database which then was used to represent the undersampled image, as is shown in Figure 4.5 (c). However in case II, the algorithm recognised a set of similar images in the database to fill in the undersampled $k$-space data, with the result being shown in Figure 4.5 (f).

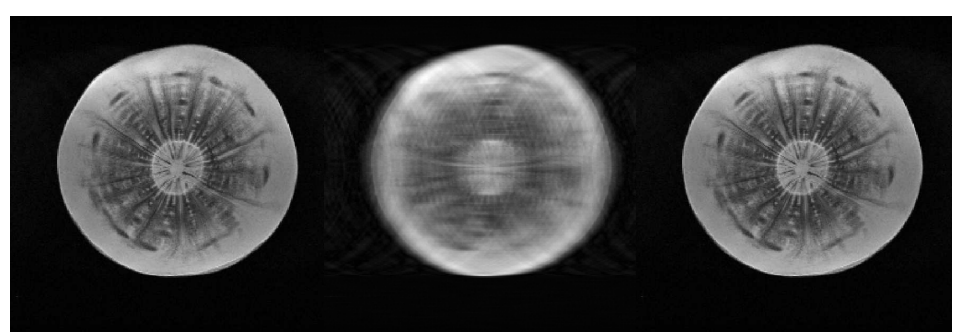

(a)

(b)

(c)

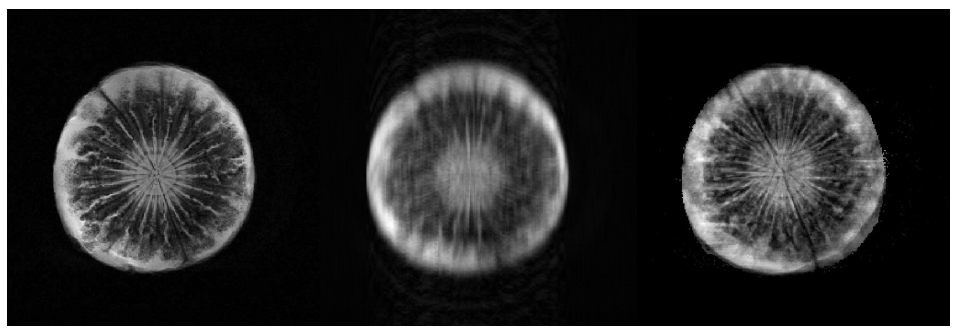

(d)

(e)

(f)

Figure 4.5: 1D-PCA-RR reconstruction results of case I and case II: (a) the full image included in database; (b) the undersampled image in case I (PSNR $=22.5)$; (c) 2D-PCA-RR reconstructed images in case I (PSNR = 319.6); (d) the full image excluded in database; (e) the undersampled image in case II (PSNR = 19.1); (f) 2D-PCA-RR reconstructed images in case II (PSNR $=26$ ).

As mentioned in the methodology part, the degree of similarity between images under reconstruction with respect to the information contained in the database can be quantitatively represented by the calculation of the 
Euclidean distance in 1D-PCA. As a consequence, the reconstructed results vary with the quality of the database, and the number of matched images to be chosen. The relationship of PSNR and the number of matched images in the two cases are shown in Figure 4.6. In case I (Figure 4.6 (a)), it is noticeable that PSNR of the reconstructed image was the highest (319.62) when one matched image was in use. A similar PSNR value is seen when the number of matched images is either two or three, simply meaning that there were two images in the database which had similar distances to the undersampled image. If more than three images were matched, a steady decrease in PSNR is seen. When more than twenty images were matched, a plateau of PSNR approximately equal to 22.5 is observed in Figure 4.6 (a), which is the same level as the initial input (Figure 4.5 (b)).

In case II (Figure 4.6 (b)), PSNR was continuously increasing with the number of matched images until PSNR reached 26, when the number of matched images was twelve. After that, PSNR decreased and then remained at the same value which was slightly higher than the initial input (Figure 4.5 (e)). As the image covariance matrix in 1D-PCA depends on the database, the optimal number of matched images relies on the quality of the database and can be determined while the iteration procedure is scanning the database.

\subsection{D-PCA Recognition Reconstruction}

As discussed in Section 3.4.2, 2D-PCA is faster and occupies less computer memory than 1D-PCA, because images in the database are not required to be re-sized to vectors [158]. Therefore, it is intuitive to replace 1D-PCA part in the aforementioned recognition algorithm, offering a new reconstruction method 2D-PCA-RR. 


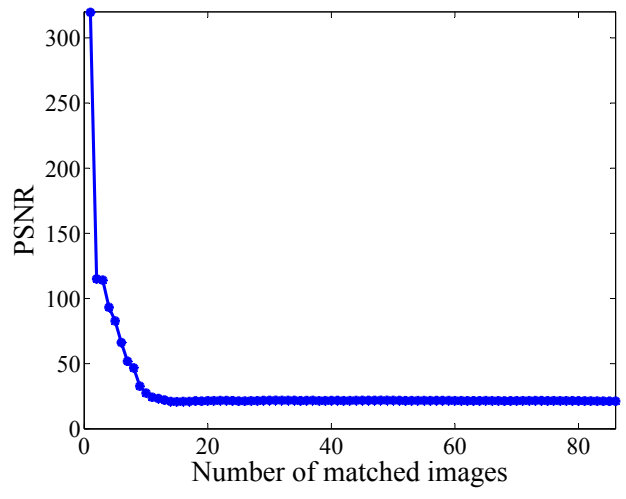

(a)

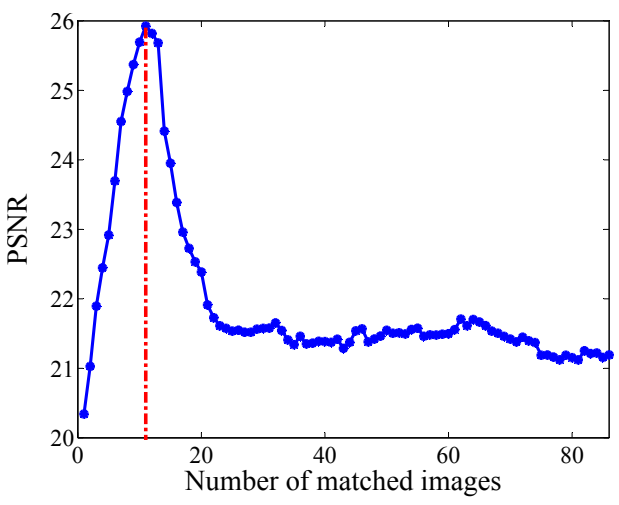

(b)

Figure 4.6: The relationship of PSNR and number of matched images in two cases: (a) case I: the full image of the undersampled image was included in the database; (b) case II: the full image of the undersampled image was excluded in the database.

\subsubsection{Methodology}

This algorithm directly projects the 2D matrix of undersampled image $\mathbf{I}_{\mathrm{u}}$ on the eigenvector matrix $\mathbf{U}$ and compares the resulting $\left(\mathbf{P C}^{\prime}\right)$ with the principal component database. The only difference from 1D-PCA-RR is that the recognition procedure uses the sum over the Euclidean distances of the column vectors from the matrices $\mathbf{P C}^{\prime}$ and $\mathbf{P C}^{i}$ as selection criterion

$$
d_{e}^{i}=\sum_{i=1}^{M} \sqrt{\sum_{i=1}^{N}\left(P C_{k l}^{\prime}-P C_{k l}^{(i)}\right)^{2}}
$$

It is worth mentioning that the way of calculating the distance in Equation (4.4) diverges from the calculation of the Euclidean counterpart between the two matrices $\left(=\sqrt{\sum_{i=1}^{M} \sum_{i=1}^{N}\left(P C_{k l}^{\prime}-P C_{k l}^{(i)}\right)^{2}}\right)$. This is due to the fact that each column vector in $\mathbf{P C}^{\prime}$ and $\mathbf{P C} \mathbf{C}^{i}$ represents an independent feature in the 2D images [158], and the kernel of the recognition is not to calculate how close two matrices (images) are globally, but to determine whether individual features are similar locally. Therefore, it is crucial to 


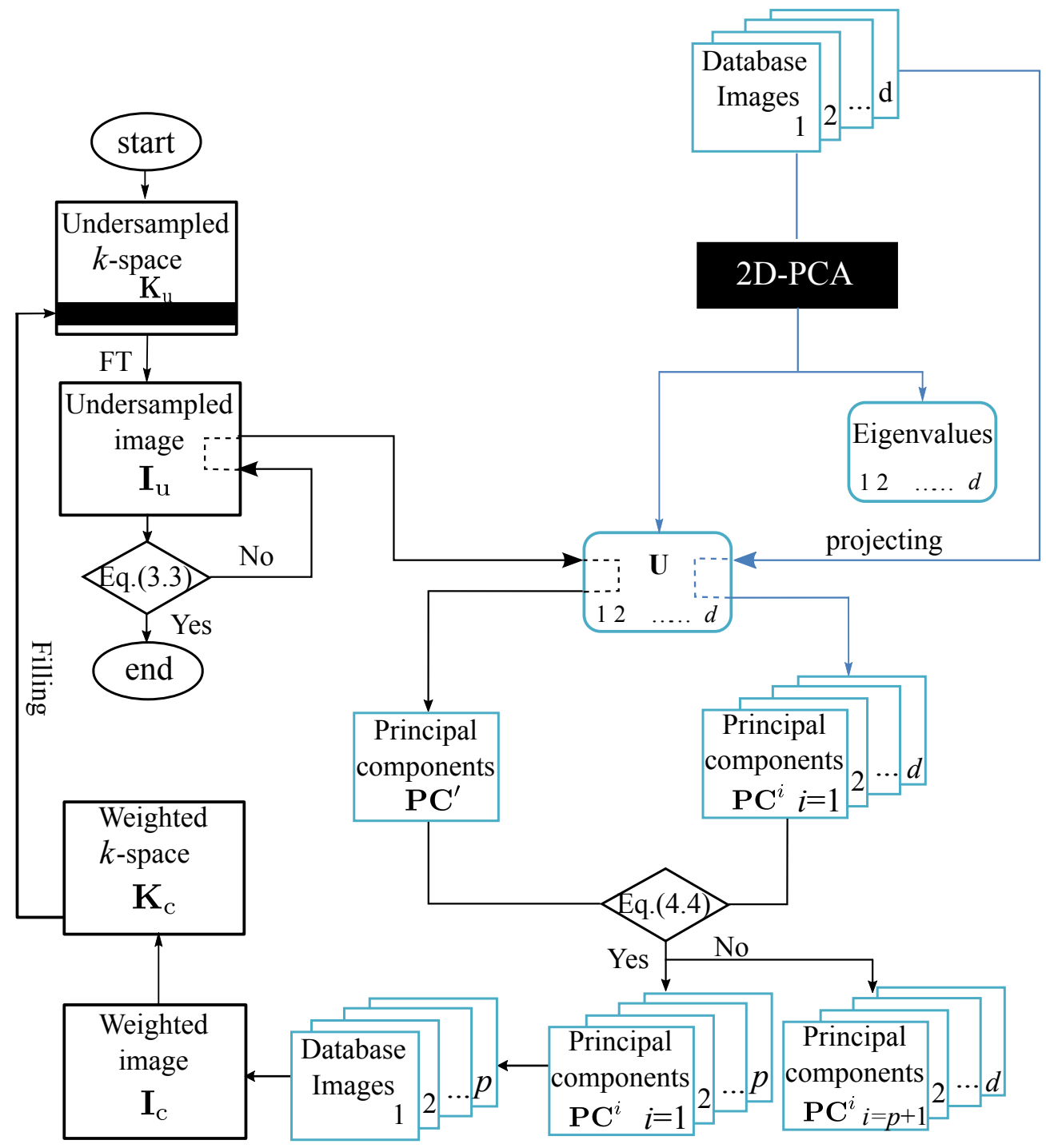

Figure 4.7: Flow chart of 2D-PCA-RR. The black box is the procedure of 2D-PCA. The procedure outside the box is iterated until Equation (3.3) is satisfied. $\mathbf{P C}^{\prime}$ is the principal component matrix after projecting the undersampled image to the principal component basis. 
measure the difference between individual columns of $\mathbf{P C}^{\prime}$ and $\mathbf{P C} \mathbf{C}^{i}$ and then accumulate these differences to indicate the similarity. The degree of similarity between $\mathbf{P C}^{\prime}$ and $\mathbf{P C}^{i}$ can then be quantitatively described by $d_{e}{ }^{i}$ as defined in Equation (4.4) and thus, the most similar images in the MR database can be identified and combined to represent the image under study.

Figure 4.7 illustrates the procedure of 2D-PCA-RR. The black box in Figure 4.7 simplifies the calculation of 2D-PCA, outputting the eigenvector matrix (U) and the corresponding eigenvalues. Subsequently, a set of principal component matrices $\left(\mathbf{P C}^{1}, \mathbf{P C} \mathbf{C}^{2}, \ldots, \mathbf{P C}^{d}\right)$ is derived by projecting all the images on the database onto $\mathrm{U}$, according to Equation (3.24). Each $\mathbf{P C}^{i}$ will then be compared with the principal components $\left(\mathbf{P C}^{\prime}\right)$ of the undersampled image $\left(\mathbf{I}_{\mathrm{u}}\right)$ through the similarity function which is defined by Equation (4.4). Based on the distances, a set of $p$ images is selected from the database, along with its corresponding principal components. In addition, the distances between the principal components are re-normalised and the selected images are added together as in Equation (4.3). The rest of the procedure is the same with 1D-PCA-RR.

It should be noted that both 1D-PCA-RR and 2D-PCA-RR require the same kind of input variable $\left(\mathbf{K}_{\mathrm{u}}\right)$. If the two methods share the same image database, the final reconstructed results of the 1D-PCA-RR and 2D-PCA-RR are the same, which were able to be confirmed using various experiments. As 2D-PCA-RR requires less computational time, further studies were based on 2D-PCA-RR in replace of 1D-PCA-RR.

\subsubsection{Comparison with CS-based algorithms}

In addition to PSNR, SSIM [185] estimates the differences of two images in terms of luminance, contrast, as well as structural changes in a user- 
defined window. SSIM [186] is defined as ${ }^{1}$

$$
\operatorname{SSIM}(\mathbf{a}, \mathbf{b})=\frac{2 \mu_{\mathbf{a}} \mu_{\mathbf{b}}+\mathbf{c}_{1}}{\mu_{\mathbf{a}}^{2}+\mu_{\mathbf{b}}^{2}+\mathrm{c}_{1}} \cdot \frac{2 \sigma_{\mathbf{a}} \sigma_{\mathbf{b}}+\mathbf{c}_{2}}{\sigma_{\mathbf{a}}^{2}+\sigma_{\mathbf{b}}^{2}+\mathrm{c}_{2}} \cdot \frac{\sigma_{\mathbf{a b}}+\mathbf{c}_{3}}{\sigma_{\mathbf{a}}+\sigma_{\mathbf{b}}+\mathbf{c}_{3}},
$$

where $\mu_{\mathrm{a}}$ and $\mu_{\mathrm{b}}$ substitute the mean value of the original and reconstructed images $\mathbf{a}$ and $\mathbf{b}$, respectively. $\sigma_{\mathbf{a}}$ and $\sigma_{\mathbf{b}}$ represent the standard deviations, and $\sigma_{\mathrm{ab}}$ is the covariance of the two images. The constants of $\mathrm{c}_{1}, \mathrm{c}_{2}$ and $\mathrm{c}_{3}$ are introduced to avoid computational error when the denominators are close to zero. SSIM varies from -1 to 1 , and only when $\mathbf{a}=\mathbf{b}$, SSIM $=1$ [186]. SSIM was calculated for a set of $11 \times 11$ windows, which were displaced pixel-by-pixel to cover the whole image. From the set of SSIM values, the mean SSIM (MSSIM) was calculated and the result used as a similarity measure between the original and reconstructed images.

The reconstructed images from 2D-PCA-RR, 1D-PCA-CS and waveletCS for case I (the image in the database) and case II (the image not in the database) are compared in Figure 4.8. The produced error images of different algorithms are shown in Figure 4.9 and the PSNR and SSIM values are summarised in Table 4.1. PSNR of the undersampled images (Figure 4.5 (b) and (e)) and reconstructed images via wavelet-CS (Figure 4.8 (d) and (h)) with a sampling rate of $20 \%$ were consistent with the results from [95].

In case I, PSNR and SSIM of the reconstructed images (Figure 4.8 (b)-(d)) using 2D-PCA-RR are the highest, followed by 1D-PCA-CS and waveletCS. Some aliasing artefacts can still be observed from the reconstructed image via wavelet-CS, while the reconstructed images via 1D-PCA-CS and 2D-PCA-RR are visually better than wavelet-CS, either in terms of the reconstructed images themselves, or the error images. Moreover, 2D-PCA$R R$ returns exactly the same image from the database, resulting in no error. This may be the case I in clinical MRI if some fully sampled images of a patient are pre-available or a patient has repeated MRI investigations of the

\footnotetext{
${ }^{1}$ The detailed implementation of the SSIM algorithm can be found in https: / / ece. uwaterloo.ca/ z70wang/research/ssim/ssim.m.
} 


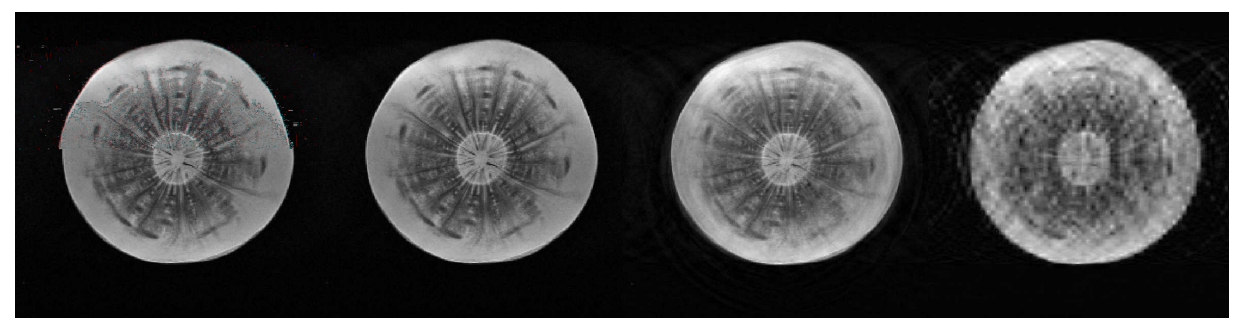

(a)

(b)

(c)

(d)

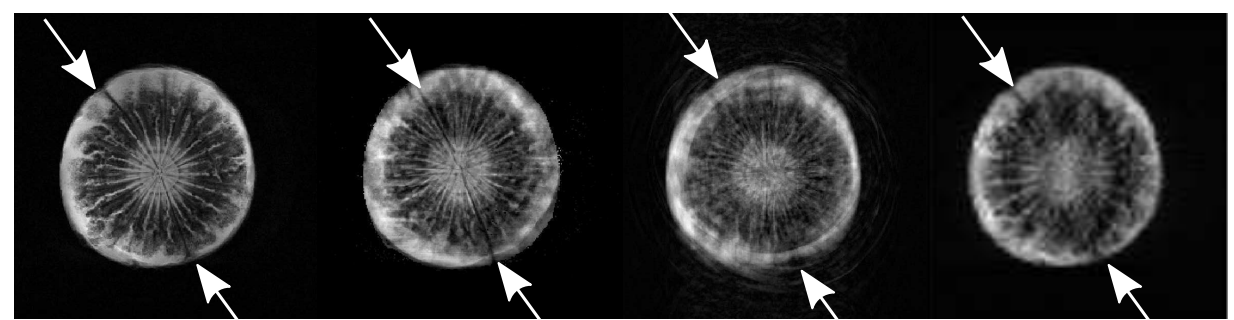

(e)

(f)

(g)

(h)

Figure 4.8: Comparison of the reconstruction methods: (a) the full image in case I; (b) 2D-PCA-RR reconstructed images in case I; (c) 1D-PCA-CS reconstructed images in case I; (d) Wavelet-CS reconstructed images in case I ; (e) the full image in case II; (f) 2D-PCA-RR reconstructed images in case II ; (g) 1D-PCA-CS reconstructed images in case II; (h) Wavelet-CS reconstructed images in case II. Arrows indicate the features that may not exist in the database.

Table 4.1: Comparison of (a) PSNR (b) SSIM values using different methods (sampling rate $=0.2$ )

(a) PSNR

\begin{tabular}{ccc}
\hline \hline Methods & case I & case II \\
\hline zero-filling FT & 22.5 & 19.1 \\
2D-PCA-RR & 319.6 & 26 \\
1D-PCA-CS & 67.3 & 21.2 \\
wavelet-CS & 34.3 & 23.7 \\
\hline
\end{tabular}

(b) SSIM

\begin{tabular}{ccc}
\hline \hline Methods & case I & case II \\
\hline zero-filling FT & 0.71 & 0.60 \\
2D-PCA-RR & 1.00 & 0.91 \\
1D-PCA-CS & 0.71 & 0.72 \\
wavelet-CS & 0.70 & 0.87 \\
\hline
\end{tabular}




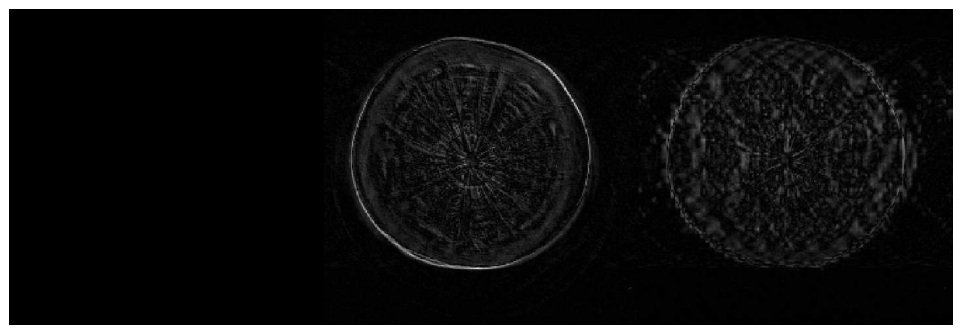

(a)

(b)

(c)

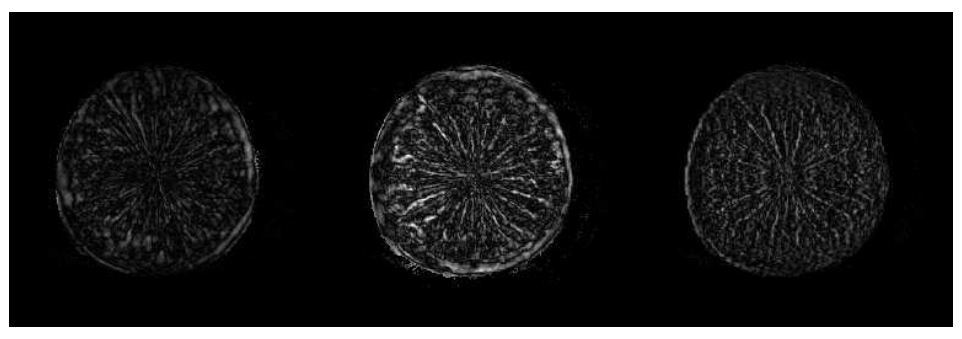

(d)

(e)

(f)

Figure 4.9: Error images of the reconstruction methods: (a) 2D-PCA-RR reconstructed images in case I; (b) 1D-PCA-CS in case I; (c) Wavelet-CS in case I ; (d) 2D-PCA-RR reconstructed images in case II ; (e) 1D-PCA-CS in case II; (f) WaveletCS in case II. 
same area. It is possible that some alterations may occur when a patient has more than one scan, such as tissue composition or stiffness changes. These differences will result in different signal intensity distributions in the images, and will be discussed in detail in Section 4.4.3. The error image from 1D-PCA-CS shows that this algorithm is capable of reconstructing the correct contrast of the eptimis tissues. The error image from wavelet-CS still presents some blurring features, resulting from the insufficient sparsity of wavelet to the particular case I image.

In case II, PSNR and SSIM of the reconstructed images (Figure 4.8 (f)(h)) using 2D-PCA-RR are the highest, followed by wavelet-CS and 1DPCA-CS. Furthermore, 2D-PCA-RR still reveals unique features (indicated by arrows in Figure 4.8 (e)-(h)) which might not exist in the database. The reconstructed image via wavelet-CS maintains the unique features because this method is independent of the image database and only relies on the pre-defined wavelet forms. While 1D-PCA-CS performed better than wavelet-CS in case I (the image in the database), PSNR and SSIM of case II (the image not in database) indicate superior performance by wavelet-CS. However, wavelet-CS is known to fail when reconstructing circular shapes and curves [95], while PCA-based methods were found to perform better in this regard. This behaviour is clearly observed when comparing the central parts of the taproots in Figure 4.8 (f)-(h) and Figure 4.9 (d)-(f).

Both 1D-PCA-CS and 2D-PCA-RR rely on the image database, while wavelet-CS is irrelevant to it. As a result, wavelet-CS may have more advantages in reconstructing the images if the database is of low quality. However, this performance gain may vanish if the database itself is self-learning (e.g. by adding rotated and realigned images already existing in the database, or adding more examination results as pointed out in [171]), thus increasing the probability of having more similar images in the database over time.

The number of iterations (necessary for image reconstruction in case II) is compared for the three reconstruction methods and the results are shown 
in Figure 4.10. 2D-PCA-RR needed the smallest number of iterations converging to the ultimate result, which was five iterations in total. Whilst 1D-PCA-CS needed two more iterations and the wavelet-CS required quadrupled iterations than 2D-PCA-RR to converge. Therefore, comparing the rates of convergence, 1D-PCA-CS and 2D-PCA-RR are more efficient options to reconstruct the undersampling $\mathrm{MR}$ images. In addition, the reconstruction time for obtaining the same results with already optimised parameters requires $1.04 \mathrm{~s}, 1.12 \mathrm{~s}$ and $2.43 \mathrm{~s}$ for 2D-PCA-RR, 1D-PCA-CS and wavelet-CS, respectively. This demonstrates 2D-PCA-RR to be most time-efficient.

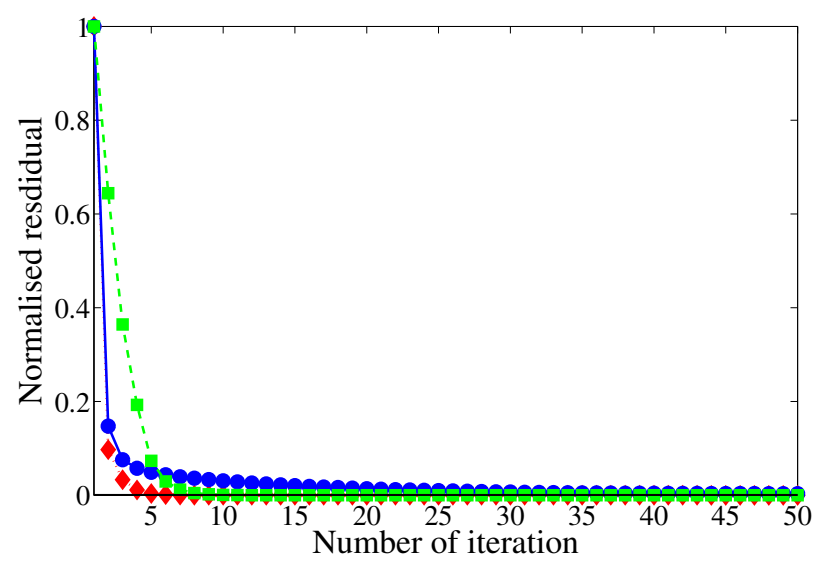

Figure 4.10: Comparison of number of iterations between different reconstruction methods when the full $k$-space information of undersampled image is excluded

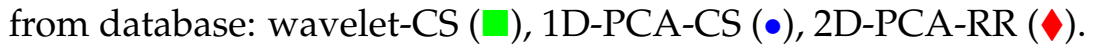

The relationship between the sampling rates and PSNR values of the different reconstruction methods in case II is shown in Figure 4.11. Not surprisingly, PSNR increases with increasing sampling rate, in other words, with a reduced amount of undersampling. For sampling rates lower than 0.6, PSNR of 2D-PCA-RR is the highest, while wavelet-CS presents the highest PSNR for sampling rates above 0.6. The PSNR value of 1D-PCA$\mathrm{CS}$ is smaller than the value obtained with wavelet-CS for sampling rates larger than 0.2. When the sampling rate is lower than 0.2, PSNR of 1D-PCA- 
CS, wavelet-CS and zero-filling FT are comparable, while 2D-PCA-RR still performs reasonably better. This clearly shows the power of 2D-PCA-RR reconstruction method when the image is highly undersampled.

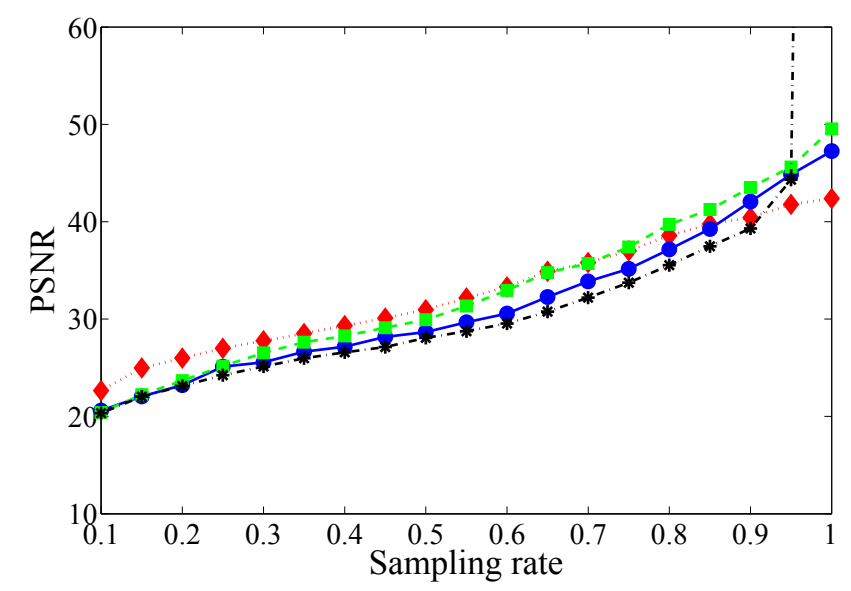

Figure 4.11: The relationship between PSNR values and sampling rates for different reconstruction methods in case II: 2D-PCA-RR $(\diamond)$, wavelet-CS ( $\square)$, 1D-PCA-CS $(\bullet)$ and zero-filling FT $(*)$.

\subsubsection{Image with alterations}

As discussed in Section 4.3.2, case I may emulate the situation where a patient's fully sampled MRI scan is pre-available in the database. As there might be some alterations due to specific medical conditions, the new image may not be in the database, so strictly speaking it falls into case II. However, most of the features in the image are preserved in the database, only localised changes may occur in the image but are not registered in the database. In order to investigate the response of different reconstruction methods to this scenario, a Gaussian mask was applied to the fully sampled image as shown in Figure 4.8 (a) to simulate localised tissue changes in biological samples. Such modified fully sampled image with the Gaussian mask is given in Figure 4.12 (a). It should be pointed out that this alteration of the image is not part of the database. When applying the 
random undersampling pattern (Figure 3.5) in $k$-space, the reconstructed image by zero-filling FT is illustrated in Figure 4.12 (b). The reconstructed images by using 2D-PCA-RR, 1D-PCA-CS and wavelet-CS are presented in Figure 4.12 (c), (d) and (e) respectively. Error images with zero-filling FT, 2D-PCA-RR, 1D-PCA-CS and wavelet-CS are shown in Figure 4.13. While 2D-PCA-RR performs best and returns the highest PSNR, the result is still based on only one image (the unaltered one in the database) as selected by the algorithm. In addition, 1D-PCA-CS performs better than wavelet-CS for this case of image alterations.

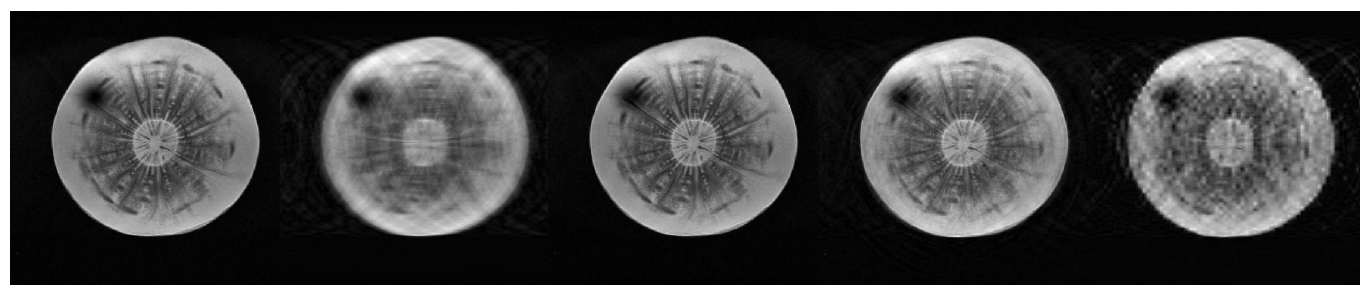

(a)

(b)

(c)

(d)

(e)

Figure 4.12: Comparison of the reconstruction methods: (a) the altered image in case I; Reconstructed images using (b) zero-filling FT (PSNR = 19.9).; (c) 2DPCA-RR (PSNR = 42.5); (d) 1D-PCA-CS (PSNR = 26.1); (e) Wavelet-CS (PSNR = 22.4).

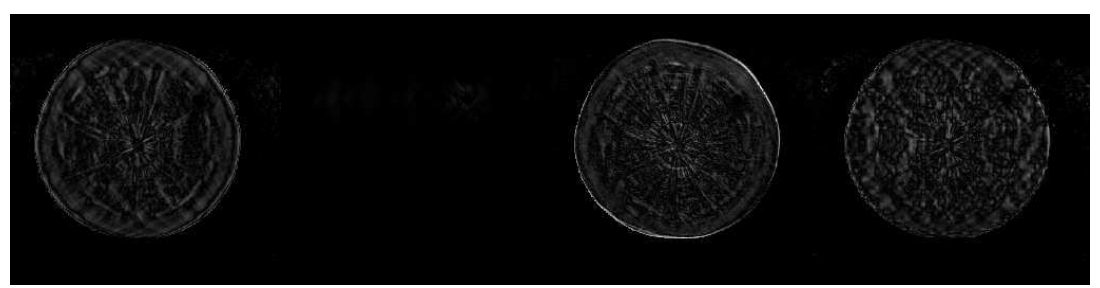

(a)

(b)

(c)

(d)

Figure 4.13: Error images of the reconstruction methods: (a) zero-filling FT; (b) 2D-PCA-RR; (c) 1D-PCA-CS; (d) Wavelet-CS.

The relationship of PSNR values and sampling rates for the case of image alteration is depicted in Figure 4.14. PSNR of 2D-PCA-RR is the highest for the entire range, whereas, PSNR of wavelet-CS is higher than 
1D-PCA-CS when the sampling rate is larger than 0.8. However, when the sampling rate decreases below $0.8,1 \mathrm{D}-\mathrm{PCA}-\mathrm{CS}$ performs better than wavelet-CS. Therefore, it can be concluded that the reconstruction methods based on PCA are still capable of returning better results compared to wavelet-CS which is not bound to any image database and thus lacks prior knowledge.

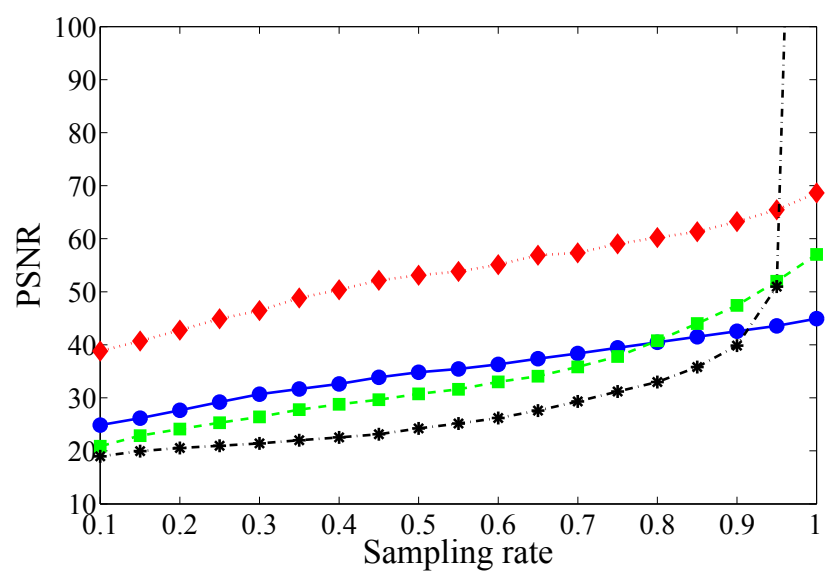

Figure 4.14: The relationship between PSNR values and sampling rates for different reconstruction methods in the case of an altered image (case III): 2D-PCA-RR $(\diamond)$, wavelet-CS $(\square)$, 1D-PCA-CS $(\bullet)$ and zero-filling FT $(*)$.

Apart from the simulated alterations, other types of changes may exist when repeating scans. For example, the image of the initial scan is in the database, but the subsequent scans have rotational and translational changes. This has two solutions: one is that the database is trained to include the rotated or translated images, which is simple but requires more computer memory and search time when executing PCA (both 1D and 2D). The other solution is including rotational or translational matching into the algorithm during the recognition procedure, where a localised feature can be extracted and convoluted with images (vector-based) in the database. Such algorithms are available, however it is beyond the scope of this thesis to study their joint implementation with the proposed algorithm.

The comparison of different cases suggests that the overall performances 
of PCA-based reconstructions (including 1D-PCA-CS, 1D-PCA-RR and 2DPCA-RR) are better than the wavelet-CS method for case I followed by case III. Case II still allows the PCA-based algorithms to recover the image with small gains in performance as compared to wavelet-CS. Going with the undersampled image even further away from the class of objects included in the database (for instance, using the carrot database to reconstruct an undersampled image of an apple), the proposed approaches may have little or negative improvements.

While the handling of a mask based on Cartesian coordinates is more straightforward as compared to other undersampling patterns (e.g. radial or variable density spiral sampling) it is known to be more sensitive to certain image artefacts (such as patient movements or gradient induced vibrations) $[62,63]$. More advanced sampling schemes may potentially reduce the required number of iterations during reconstruction and improve the overall performance of the algorithm.

It is worth mentioning that this procedure as studied uses a database containing MRI intensities. Therefore, it is restricted to the processing of proton density distributions and their contrasts. However, if $T_{1}, T_{2}$ or $D$ data is included in the database as well, the presented methods may lend itself to process a wide set of undersampled $k-t$ or $k-b^{2}$ data as has been investigated by several pioneers [164-167, 169, 170, 187]. Moreover, due to the merit that the PCA-based recognition algorithms are not relying on particular NMR parameters, it has the potential to be applied to other imaging techniques, such as ultrasound tomography [188].

\subsubsection{Different database}

In order to corroborate the applicability of our algorithm a set of crosssectional $T_{1}$-weighted MRI scans of healthy brains retrieved from OASIS database [189] were used for comparison. The algorithms for cases I and

\footnotetext{
${ }^{2}$ DWI data, by analogy with $k-t$.
} 
II were implemented on this database, among which the reconstructions showed similar trend to the carrot database, 2D-PCA-RR had the best performance, while 1D-PCA-CS and wavelet-CS competed each other depending on whether the fully sampled image existed in the database or not. More importantly, a Gaussian mask was also applied on one of the brain images to simulate a hemorrhagic infarct, similarly to how an image alteration was done for case III using the carrot database.

Figure 4.15 shows the results for the $20 \%$ sampling rate image and the corresponding reconstructed and error images of 2D-PCA-RR and waveletCS. Although the error image of 2D-PCA-RR reconstruction shows that the Gaussian mask area was not fully recovered, it still returned an improved image as compared to Figure 4.15 (a) and (d). In the meantime, waveletCS retrieved higher PSNR compared to the direct zero-filling FT method. However, detailed features are more distorted as compared to 2D-PCA-RR as shown in Figure 4.15 (c) and (f).

\subsection{Conclusions}

A CS scheme based on a sparse transformation domain utilising principal components (1D-PCA-CS) was adapted to MRI. In addition, new reconstruction algorithms (1D-PCA-RR and 2D-PCA-RR) for highly undersampled MR images were introduced in this chapter. When $k$-space is undersampled (as low as 20\%), it is important to draw on prior knowledge for the image under reconstruction. The three methods utilise the merits of a self-learning database and shares the benefits of reduced acquisition time as typical for CS schemes. The experimental results of undersampled images for carrot taproots were shown in this chapter for three cases; Case I was when the fully sampled image was included in the database and case II was that the fully sampled image was excluded from the database. In case I, 1D-PCA-RR and 2D-PCA-RR returned the exact image from the database leading to the highest PSNR, and 1D-PCA-CS showed better res- 


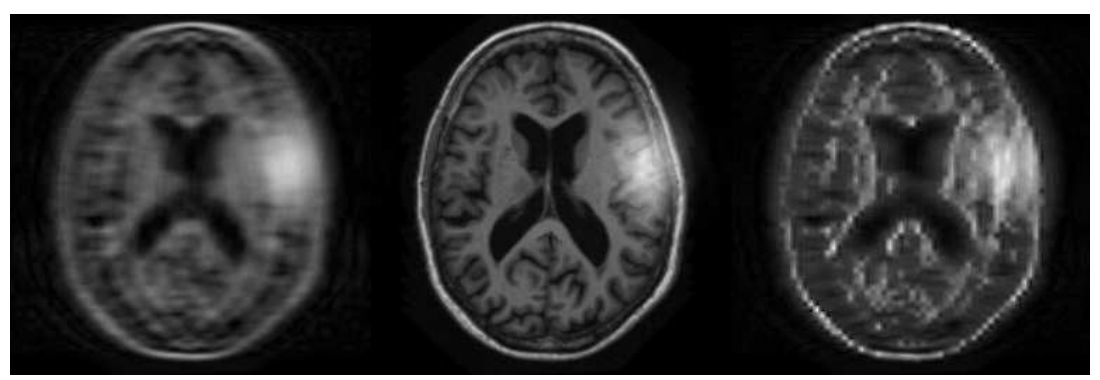

(a)

(b)

(c)

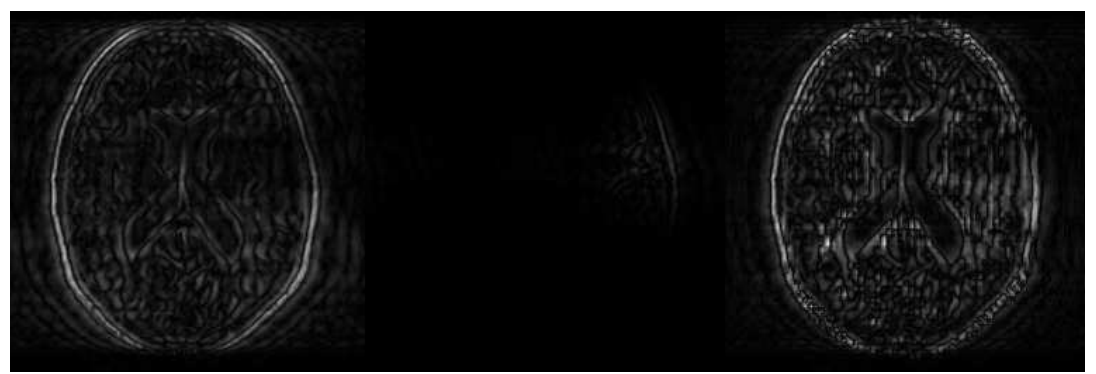

(d)

(e)

(f)

Figure 4.15: Comparison of the reconstruction methods by using the brain dataset: (a) zero-filling FT (PSNR = 21.6); (b) 2D-PCA-RR (PSNR = 33.3); (c) Wavelet-CS $(P S N R=22.5)$. Error images are shown below in parallel with the reconstructed image. 
ults than wavelet-CS. In case II, PCA-RR methods still performed better due to prior knowledge of similar images in the database. 1D-PCA-CS performed similar to wavelet-CS for case II. In the meantime, with the increase of sampling rate, using wavelet-CS as the reconstruction method could achieve higher PSNR than using PCA-based methods. However, the performance of 1D-PCA-CS might improve over time due to self-learning capabilities of the database. Moreover, in the case of a locally altered image (case III) PCA-based methods performed superior over wavelet-CS for sampling rates below 0.8 . As a consequence, the experimental results of the three cases as well as the brain dataset demonstrated the speed and feasibility of PCA-based methods in MRI with sampling rates as low as 0.2. It is noticeable that the performances of PCA-based methods rely on the quality of the database, which means that the training of the database is essential before applying these two methods. While only proton density distributions were reconstructed in this chapter, these methods could be extended to recover $T_{1}, T_{2}$ or $D$ information and have the potential to be applied to more general imaging techniques. 


\section{Chapter 5}

\section{Tissue Anisotropy Determination by NMR Spectroscopy}

The structures of biological tissues are complex. One important parameter for characterising their morphology is the degree of anisotropy. This chapter offers an alternative way of determining the fractional anisotropy as a sample average. It is based on an established NMR diffusometry protocol-DDCOSY-in conjunction with an appropriate gradient scheme. Consequently, mean FA values obtained from biological tissues are compared with DTI results at the end of this chapter. 


\subsection{Introduction}

As introduced in Section 3.2.2, FA [31] can quantitatively characterise orientation dependence of molecular mobility, which enables the differentiation of compartment shapes or identify pathological changes in tissues. For instance, in a material with an internal structure (e.g. a spherical pore or compartment filled with fluids) returns a FA value of zero due to isotropic diffusion. However, anisotropic diffusion exhibits a FA value between zero and one. In biological tissues, interior fibre structures and cell alignments result in different FA values. It has been reported that FA in breast cysts was smaller in comparison to the surrounding healthy tissues [190]; The changes of FA in the central nervous system due to the disordering of the fibres can be indicators of abnormalities such as stroke [191]. Therefore, this concept has been widely studied and used in structural biology, material science and medicine [192-195].

Spatially resolved FA is usually obtained by further processing DTI data [34]. However, a trend has been shown to utilise sample-averaged FA in the study of the post-natal development of mouse brain at various ages, as carried out by Larvaron and co-workers [196]. In their study, data was acquired using DTI initially, followed by averaging over all pixels. This processing step would be obsolete if the NMR signal was measured using a spectroscopic method returning the response from the whole sample volume, thus directly yielding the mean value of FA. As already mentioned in Section 3.3.1, DDCOSY will be an appropriate choice for this purpose. While early applications to chive plants returned signatures of cell shapes [148], its potential to quantitatively extract sample-averaged FA was so far not discovered.

A new approach which combines the conventional DDCOSY scheme with the strategy learnt from DTI will be introduced in this chapter. Throughout numerical simulations on fibres and experiments on three biological samples, the new approach will show its capability of obtaining bulk dif- 
fusion tensor elements and subsequent mean FA values as quantitative progress.

\subsection{Methodology}

\subsubsection{From DTI to DDCOSY}

In order to obtain the six uncorrelated elements of the bulk diffusion tensor (i.e. averaged over the sample volume), a novel scheme is presented in this section to combine three DDCOSY experiments with gradient orientations as depicted in Figure 5.1. While $\mathbf{q}_{1}$ is the gradient wave-vector along the main axis in the laboratory (Cartesian) system $\left(x-, y-\right.$, or $z-$ axis), $\mathbf{q}_{2}$ is the gradient wave-vector on the plane $(y z-, x z-$, or $x y-$ plane) with an off-axis angle of $\theta$. If the directions of the gradient pairs follow Figure 5.1 (a), the exponential factor in the first dimension in Equation (3.17) can be expanded as:

$$
\begin{aligned}
\mathbf{q}_{1}^{\mathrm{T}} \mathbf{D}_{\mathbf{1}} \mathbf{q}_{1} \Delta_{1} & =\left(\begin{array}{c}
q_{1} \\
0 \\
0
\end{array}\right)^{\mathrm{T}}\left(\begin{array}{ccc}
D_{x x} & D_{x y} & D_{x z} \\
D_{y x} & D_{y y} & D_{y z} \\
D_{z x} & D_{z y} & D_{z z}
\end{array}\right)\left(\begin{array}{c}
q_{1} \\
0 \\
0
\end{array}\right) \Delta_{1} \\
& =\left(\begin{array}{c}
q_{1} D_{x x} \\
q_{1} D_{x y} \\
q_{1} D_{x z}
\end{array}\right)\left(\begin{array}{c}
q_{1} \\
0 \\
0
\end{array}\right) \Delta_{1} \\
& =q_{1}^{2} D_{x x} \Delta_{1} .
\end{aligned}
$$

The comparison between Equation (3.18) and Equation (5.1) returns the relationship of

$$
D_{1}^{a p p}=D_{x x}
$$

in the first dimension. However, because the second gradient pair is not applied on the coordinate axis, the expansion of the exponential factor 
in the second dimension will be more complex as compared to the first dimension:

$$
\begin{aligned}
\mathbf{q}_{2}^{\mathrm{T}} \mathbf{D}_{2} \mathbf{q}_{2} \Delta_{2} & =\left(\begin{array}{c}
0 \\
q_{2} \cos \theta \\
q_{2} \sin \theta
\end{array}\right)^{\mathrm{T}}\left(\begin{array}{ccc}
D_{x x} & D_{x y} & D_{x z} \\
D_{y x} & D_{y y} & D_{y z} \\
D_{z x} & D_{z y} & D_{z z}
\end{array}\right)\left(\begin{array}{c}
0 \\
q_{2} \cos \theta \\
q_{2} \sin \theta
\end{array}\right) \Delta_{2} \\
& =\left(\begin{array}{l}
q_{2} \cos \theta D_{y x}+q_{2} \sin \theta D_{z x} \\
q_{2} \cos \theta D_{y y}+q_{2} \sin \theta D_{z y} \\
q_{2} \cos \theta D_{y z}+q_{2} \sin \theta D_{z z}
\end{array}\right)\left(\begin{array}{c}
0 \\
q_{2} \cos \theta \\
q_{2} \sin \theta
\end{array}\right) \Delta_{2} \\
& =q_{2}^{2}\left(D_{y y} \cos ^{2} \theta+D_{z z} \sin ^{2} \theta+2 D_{y z} \cos \theta \sin \theta\right) \Delta_{2} .
\end{aligned}
$$

Thereafter, the equality of Equation (5.3) and Equation (3.18) gives the formula of the apparent diffusion coefficient in the second dimension, which is

$$
D_{2}^{a p p}=D_{y y} \cos ^{2} \theta+D_{z z} \sin ^{2} \theta+2 D_{y z} \cos \theta \sin \theta .
$$

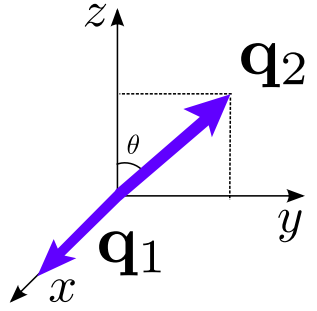

(a) $x-y z$

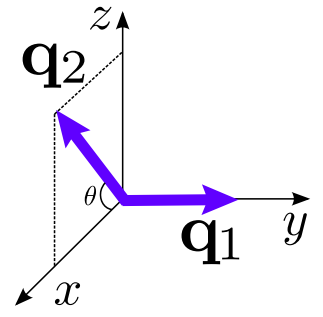

(b) $y-x z$

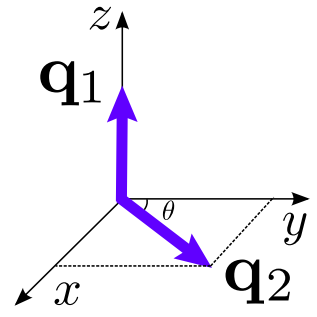

(c) $z-x y$

Figure 5.1: Diagram of the gradient directions in the three independent DDCOSY experiments. Diffusion gradient pairs $\left(\mathbf{q}_{1}\right.$ and $\left.\mathbf{q}_{2}\right)$ applied along (a) $[1,0,0]$ and $[0, \sin \theta, \cos \theta] ;(b)[0,1,0]$ and $[\cos \theta, 0, \sin \theta] ;(c)[0,0,1]$ and $[\sin \theta, \cos \theta, 0]$, respectively.

Similarly, it can be worked out that gradient directions in Figure 5.1 (b) 
return apparent diffusion coefficients as

$$
\begin{aligned}
& D_{1}^{a p p}=D_{y y}, \\
& D_{2}^{a p p}=D_{x x} \cos ^{2} \theta+D_{z z} \sin ^{2} \theta+2 D_{x z} \cos \theta \sin \theta ;
\end{aligned}
$$

and Figure 5.1 (c) gives:

$$
\begin{aligned}
& D_{1}^{a p p}=D_{z z}, \\
& D_{2}^{a p p}=D_{x x} \cos ^{2} \theta+D_{y y} \sin ^{2} \theta+2 D_{x y} \cos \theta \sin \theta .
\end{aligned}
$$

Consequently, in the three DDCOSY experiments, only one diagonal matrix element $D_{i i}$ contributes to the signal decay in the first dimension. However, signal decay in the second dimension includes one off-diagonal element $D_{i j}(i \neq j)$ besides the remaining two other diagonal elements. A convenient choice for $\theta$ is $\pi / 4$ such that the apparent diffusion coefficient in the second dimension can be calculated as:

$$
D_{i j}^{a p p}=\frac{D_{i i}}{2}+\frac{D_{j j}}{2}+D_{i j}
$$

If the system is macroscopically isotropic, the off-diagonal elements equal to zero $\left(D_{i j}=0\right)$ and the diagonal elements are identical $\left(D_{i i}=D_{j j}\right)$, thus delivering the relationship of $D_{2}^{a p p}=D_{i i}=D_{j j} \neq 0$.

\subsection{Simulation}

\subsection{1 $\quad D-D$ maps with gradients along laboratory axes}

In order to study $D-D$ maps for different cases, Monte-Carlo simulation is implemented in Matlab to model NMR responses of water diffusion through different networks $[197,198]$. It is a computational algorithm that obtains numerical results from repeated random motions of tracers, which is different from the approach to use analytic solution to model the coupled 
pore system as described by Schwartz and co-workers [199].

In Monte-Carlo simulation, each tracer is considered to carry a magnetisation. The continuous displacement is decomposed into discrete steps, and the new position of a random-walk tracer after a time interval $d t$ depends on the previous position $\mathbf{r}_{n}(t)$ yielded by

$$
\begin{aligned}
& r_{n}^{x}(t+d t)=r_{n}^{x}(t)+d r \cdot \cos \theta \sin \phi, \\
& r_{n}^{y}(t+d t)=r_{n}^{y}(t)+d r \cdot \sin \theta \sin \phi, \\
& \left.r_{n}^{z}(t+d t)=r_{n}^{(} t\right)+d r \cdot \cos \phi,
\end{aligned}
$$

where, the walk step is $d r=\sqrt{6 \cdot D \cdot d t}, \cos \theta$ and $\phi$ are randomly chosen in the range of $[-1,1]$ and $[0, \pi]$, respectively. The tracer displacement $d r$ in each step is required to be smaller than surrounding geometric length scales to obtain effective responses. During the random walk simulation, the rule of elastic collision was adopted when the molecule hit the solid matrix (sphere) or the simulated volume wall.

Once the tracer is put into the simulated volume, the DDCOSY pulse sequence is applied to manipulate its magnetisation. In order to better illustrate the procedure, the pulse sequence of DDCOSY experiments shown in Figure 3.15 is simplified in Figure 5.2. It only contains the timing of the effective PFGs. The negative signs of the second PFG in the pairs are caused by the refocusing RF pulses.

Given an applied PFG, the phase shift of the tracer is proportional to the dot product of the displacement and the field gradient:

$$
\phi_{n}(t+d t)=\gamma \mathbf{G} \cdot \mathbf{r}_{n}(t+d t) d t
$$

It should note here that if there is no PFG applied (e.g. from $t_{1}+\delta$ to $t_{1}+\Delta$ in Figure 5.2), there will be no phase shift during that period. Therefore, 


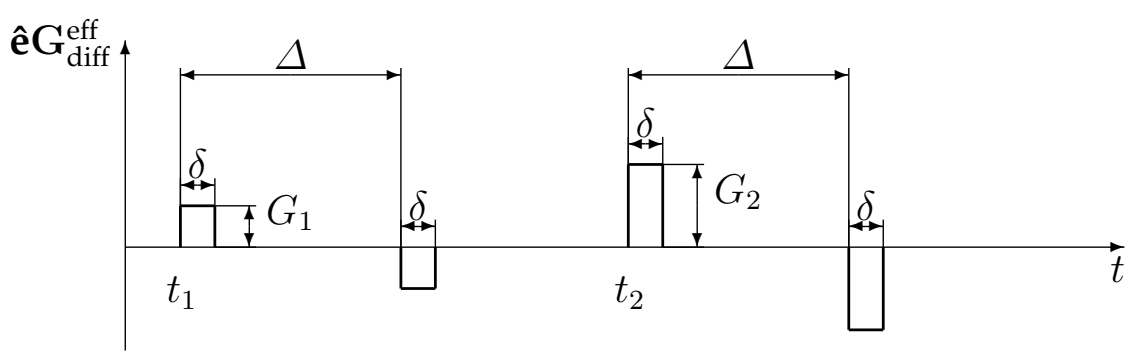

Figure 5.2: Simplified DDCOSY pulse sequence from Figure 3.15. $\mathbf{G}_{\mathrm{diff}}^{\text {eff }}$ is the effective PFG imposed on the tracers. The symbol ê in the front represents the unit vector in the direction of the respective gradient in the laboratory coordinate system. $t_{1}$ and $t_{2}$ are the starting times of the two gradient pairs, respectively.

the magnetisation of the tracer after the PFG pair is

$$
\begin{aligned}
m_{n}(t) & =m_{0} \exp \left\{-i \gamma \mathbf{G}_{1} \cdot\left[\mathbf{r}_{n}\left(t_{1}+\delta\right)-\mathbf{r}_{n}\left(t_{1}\right)-\mathbf{r}_{n}\left(t_{1}+\Delta+\delta\right)+\mathbf{r}_{n}\left(t_{1}+\Delta\right)\right]\right\} \\
\cdot & \exp \left\{-i \gamma \mathbf{G}_{2} \cdot\left[\mathbf{r}_{n}\left(t_{2}+\delta\right)-\mathbf{r}_{n}\left(t_{2}\right)-\mathbf{r}_{n}\left(t_{2}+\Delta+\delta\right)+\mathbf{r}_{n}\left(t_{2}+\Delta\right)\right]\right\},
\end{aligned}
$$

where $t_{1}$ and $t_{2}$ are the starting points of two PFGs. Therefore, the overall magnetisation can be calculated by accumulation,

$$
M(t)=\sum_{n=1}^{N_{p}} m_{n}(t)
$$

where, $N_{p}$ is the number of tracers in the space. The mean square displacement of all tracers can then be calculated by

$$
\left\langle r_{i} r_{j}\right\rangle=\frac{1}{N_{p}} \sum_{n=1}^{N p}\left[r_{n}^{i}(t)-r_{n}^{i}(0)\right]\left[r_{n}^{j}(t)-r_{n}^{j}(0)\right] .
$$

The numerical simulations were implemented in four different scenarios. One simulation scenario was modelled by a cube with the length of 2000 $\mu$ m occupied with spheres as shown in Figure 5.3 (a). The spheres constitute 
the solid matrix. Tracers were put into the simulation volume with empty space (outside the solid matrix), and randomly walk through the space. The number of tracers was set to be $N_{P}=5000$ for practical reasons ${ }^{1}$. The time interval of the discrete displacement was $d t=5 \mu \mathrm{s}$. The diffusion coefficient was set to be $D_{0}=2.5 \times 10^{-9} \mathrm{~m}^{2} / \mathrm{s}$. Thus, the length (displacement) step was $d r \approx 0.24 \mu \mathrm{m}$, much less than the characteristic length of the phantom, enabling sufficient walk steps in the geometry. The starting position of each molecule was determined by uniformly sampling points within the simulation volume to guarantee that water molecules spread the entire space in the simulated volume. The observation time $\Delta$ was set to be $100 \mathrm{~ms}$ and gradient duration $\delta$ was $2 \mathrm{~ms}$. The gradients in the two time intervals of the DDCOSY pulse sequence were applied parallel to the $x$ and $z$-axis in the laboratory coordinates. This translated into molecular displacements and the corresponding apparent diffusion coefficients after the processing tools of 2D-ILT, and are represented by the two axes shown in each of the $D$ - $D$ maps in Figure 5.3 (b). A diagonal peak is found in the $D-D$ map and the corresponding $1 \mathrm{D}$ projections are identical. As a result, the $D-D$ map indicates that the system is microscopically isotropic.

The second case was modelled by using the same cube but occupied with solid matrix modelled by fibres. The fibres were cylinders of radius $R=10 \mu \mathrm{m}$ and represented by a randomly generated pivot point and directional vector $[200,201]$. The random walk parameters were set the same as the first model. The second model and its corresponding $D-D$ maps are shown in Figure 5.4. It simulates a system which is globally isotropic but locally anisotropic (e.g. liquid-crystal, powder-like or randomly oriented fibres). From the simulated $D-D$ map, both diagonal and off-diagonal peaks are observed. 1D projections are identical, exhibiting two separated peaks. While a continuous 1D distribution (from free diffusion along the capillary axis to most restricted diffusion perpendicular to it) is expected,

\footnotetext{
${ }^{1}$ Various numbers of tracers were tested and the stable results showed the choice of 5000 was sufficient
} 


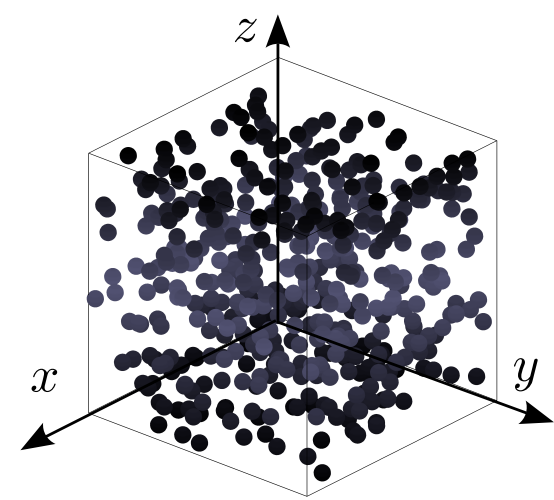

(a)

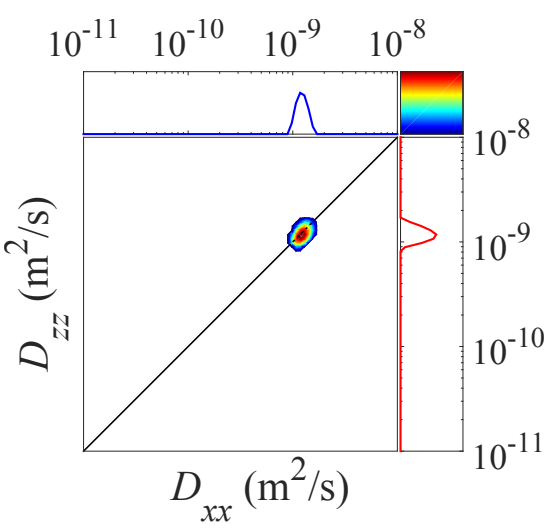

(b)

Figure 5.3: Microscopically isotropic system and its corresponding $D-D$ map, modelled by bead pack. The gradients are applied along the $x$ and $z$-axis.

the ILT returns a discrete distribution due to a "pearling" effect [133, 134], which breaks the distribution into multiple sharp peaks shown in the $2 \mathrm{D}$ plot $^{2}$.

The next simulation model kept the directions of all the fibres along $z$-axis, which is shown in Figure 5.5 (a). It illustrates a system which was globally anisotropic with no locally isotropic compartments (e.g. oriented or aligned fibres). This will be the limiting case where all cells/collagen fibres are aligned along one direction in the plant/animal tissue. Correspondingly, only off-diagonal peaks indicating inequivalent diffusion coefficient values are observed in the $D-D$ map in Figure 5.5 (b).

By tilting all fibres to a certain degree, the gradient main axes will be deviated from the main phantom geometry. If the tilting angle changes from 0 (which is the case shown in Figure 5.5) to $\pi / 4$, the off-diagonal peak will gradually move close to and even lay on the diagonal line, which were confirmed by our simulations (not shown). This indicate that single $D-D$ map is insufficient to characterise the system, which will be addressed by

\footnotetext{
${ }^{2}$ It is possible to"smooth" the distribution by using a larger regularising parameter, but that would lead to the "over-smoothing" case, which also need to be avoid when applying ILT algorithm
} 


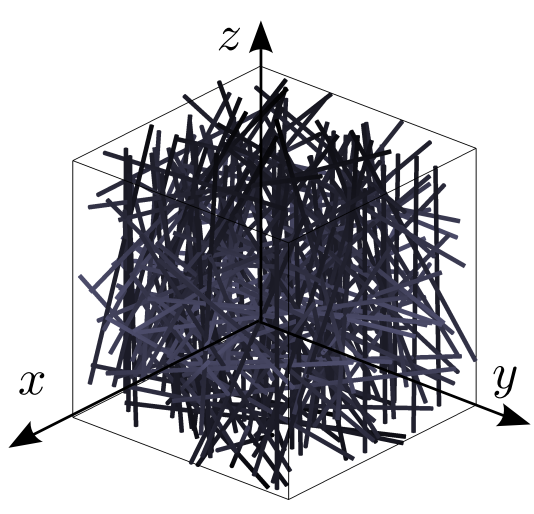

(a)

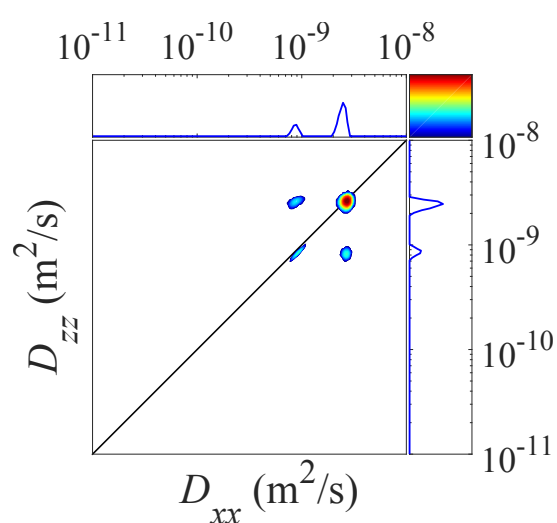

(b)

Figure 5.4: Macroscopically isotropic system and its corresponding $D-D$ map, modelled by randomly oriented fibres. The gradients are applied along the $x$ and $z$-axis.

the newly proposed approach in this thesis.

By mixing the second and third models, a system which was globally anisotropic containing isotropic components can be built. In this scenario, the degree of alignment of the fibres were randomly distributed between 0 and $90^{\circ}$. Both diagonal and off-diagonal peaks are observed in the $D-D$ map Figure 5.6 (b), but only one main off-diagonal peak is seen indicating an unsymmetrical structure. Moreover, 1D projections from $x$ - and $z$ directions are distinct. The off-diagonal peak with very small intensity might come from two reasons. They might originate from insufficient numbers of tracers (molecules) used for the simulations. Additionally, squared boundaries and the cubic simulation volume might break the cylindrical symmetry resulting in molecular displacements depending on the direction in which diffusion was observed in the $x y$ plane. Therefore, in the following simulation, the number of tracers was increased. In the meantime, the simulation volume was modified to a cylinder. 


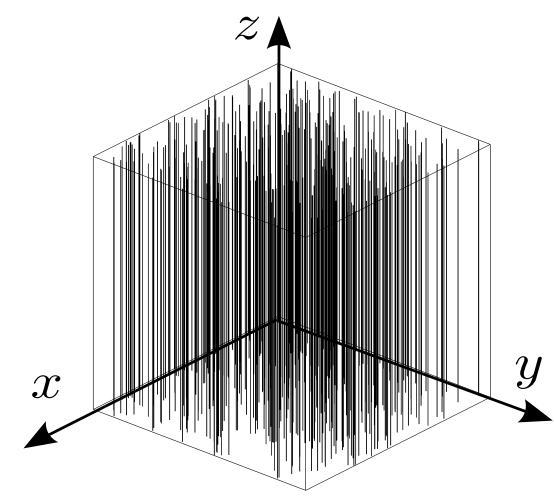

(a)

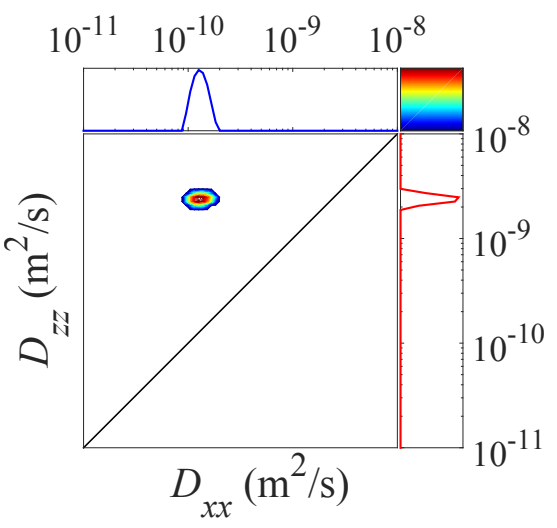

(b)

Figure 5.5: Macroscopically anisotropic system and its corresponding $D-D$ map, modelled by aligned fibres. The gradients are applied along the $x$ and $z$-axis.

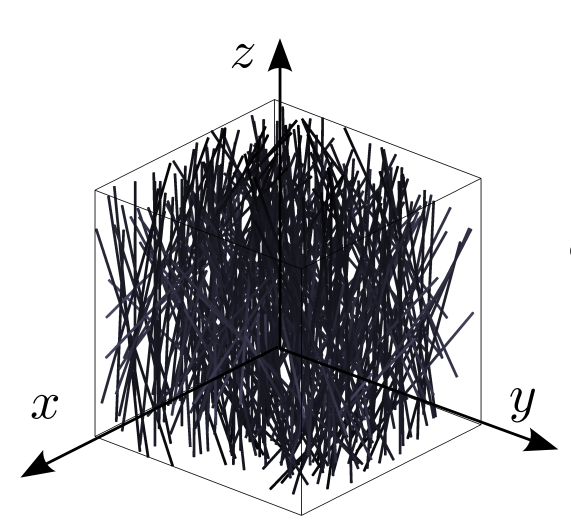

(a)

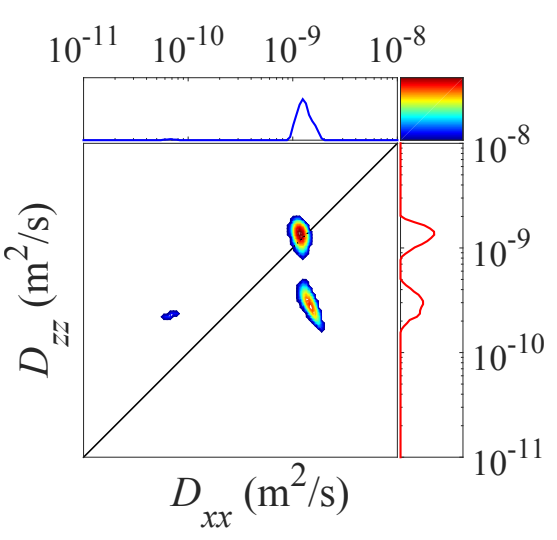

(b)

Figure 5.6: Macroscopically anisotropic system and its corresponding $D-D$ map, modelled by fibres with alignment degree randomly distributed between 0 and $90^{\circ}$. The gradients are applied along the $x$ and $z$-axis. 


\subsection{2 $D-D$ maps with the new gradient scheme}

In order to verify the concepts of obtaining FA using the proposed gradient schemes, the $D$ - $D$ maps of the three DDCOSYs were simulated. The fibre networks were modelled as shown in Figure 5.7 (a). The simulation volume was defined as a symmetrical cylinder of length $1000 \mu \mathrm{m}$ with a diameter of $600 \mu \mathrm{m}$. Tracers were placed randomly before starting the random walk through the space in Figure 5.7 (a). The number of tracers was set to be $N_{P}=7000$. Other parameters were set to the same as in Figure 5.3-5.6.

Figure 5.7 (b)-(d) are the simulated results using the proposed gradient combinations. Only one peak can be observed in the three $D$ - $D$ maps. As the system is axial symmetric, molecular diffusion is restricted to the same degree along the $x$ - and $y$-axis, leading to the similarity of Figure $5.7(b)$ and Figure 5.7 (c). A diffusion coefficient of $2.5 \times 10^{-9} \mathrm{~m}^{2} / \mathrm{s}$ (equal to the pre-set diffusion coefficient in the random walk simulation) is seen along the $z$-axis in Figure 5.7 (d), indicating that water diffusion is not restricted. As a consequence of the macroscopically anisotropic system, no diagonal peaks can be inspected in any of the $D$ - $D$ maps.

Logarithmic mean values of the diffusion coefficients in the projected 1D distribution were calculated using Equation (5.13):

$$
\log _{10}\left(D_{i j}^{a p p}\right)=\frac{\sum_{m} \log _{10}\left(D_{m}\right) \times f_{m}}{\sum f_{m}},
$$

where $D_{m}$ is the pre-defined diffusion coefficients and $f_{m}$ is the corresponding probabilities. Subsequently, the elements of the symmetric diffusion tensor matrix $\mathbf{D}_{\text {cap }}$ were reconstructed from the simulation results by extracting the logarithmic mean values from the $D-D$ maps in Figure 5.7 and using Equation (5.7): 


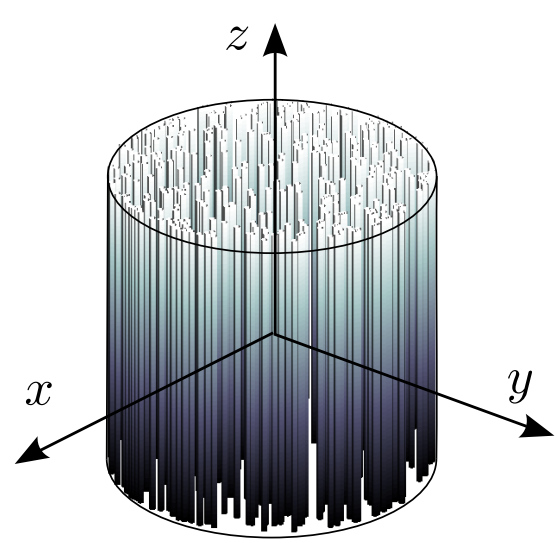

(a)

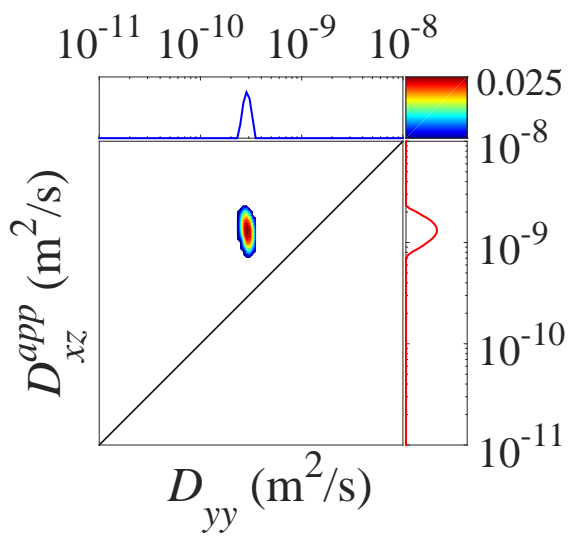

(c)

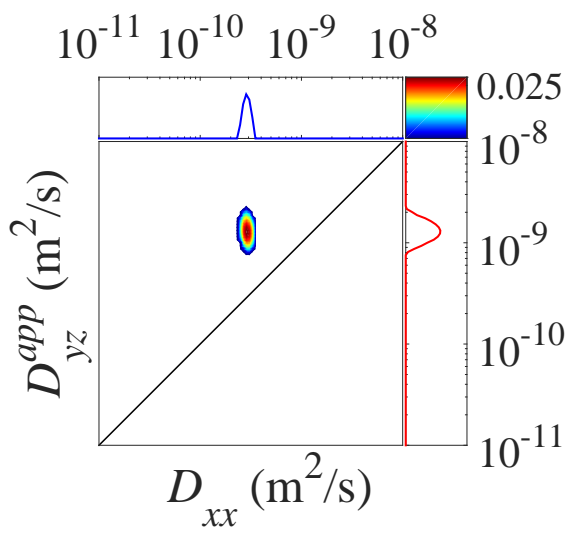

(b)

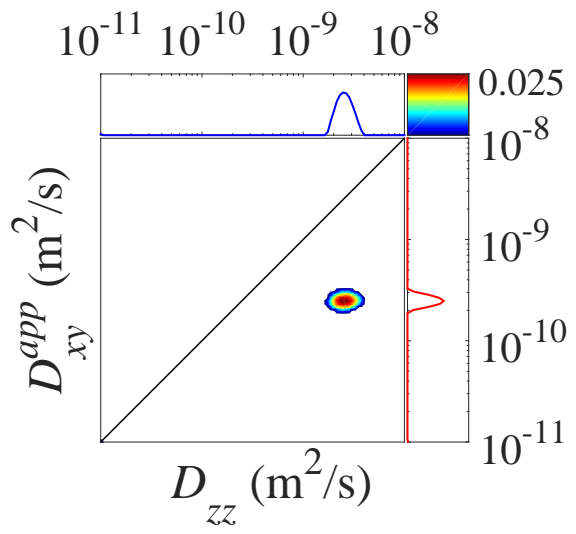

(d)

Figure 5.7: Aligned fibres in the symmetric cylinder volume and its DDCOSY simulations of the new approach using gradients combination (b) $x-y z$; (c) $y-x z$; and (d) $z-x y$. 


$$
\mathbf{D}_{\text {cap }}=\left(\begin{array}{ccc}
0.22 \pm 0.04 & 0.00 \pm 0.01 & -0.02 \pm 0.02 \\
0.0 \pm 0.01 & -0.22 \pm 0.04 & -0.01 \pm 0.02 \\
-0.02 \pm 0.02 & -0.01 \pm 0.02 & 2.35 \pm 0.07
\end{array}\right) \times 10^{-9} \mathrm{~m}^{2} / \mathrm{s}
$$

The errors of the logarithmic means were obtained from the $95 \%$ confidence interval of the ILT fitting [150].

By rotating the above diffusion tensor matrix, the eigenvectors and eigenvalues of each principal axes can be derived:

$$
\begin{aligned}
& u_{1}=(0.98,0.20,0.01)^{\mathrm{T}}, \lambda_{1}=0.22 \pm 0.10 \times 10^{-9} \mathrm{~m}^{2} / \mathrm{s} ; \\
& u_{2}=(0.19,0.98,0.00)^{\mathrm{T}}, \lambda_{2}=0.22 \pm 0.12 \times 10^{-9} \mathrm{~m}^{2} / \mathrm{s} \text {; } \\
& u_{3}=(0.01,0.00,0.99)^{\mathrm{T}}, \lambda_{3}=2.35 \pm 0.20 \times 10^{-9} \mathrm{~m}^{2} / \mathrm{s} \text {. }
\end{aligned}
$$

It is noticeable that the eigenvalues are identical to the diagonal elements of $\mathbf{D}_{\text {cap. }}$. The diffusion coefficient along $z$-axis is the largest, while the values along $x$-axis and $y$-axis are the same as expected. This further supports that the $z$-axis is the preferred pathway for water to diffuse, which is consistent with our initial model.

An alternative way to calculate the diffusion tensor is to utilise the discrete displacements which are recorded in the Monte-Carlo simulation. The ensemble mean squared displacement of all tracers can be obtained by using Equation (5.12). By further using the Einstein equation in 3D space as stated in Equation (5.16), the bulk diffusion tensor can be constructed independent of the NMR response/signal.

$$
D_{i j}=\frac{\left\langle R_{i} R_{j}\right\rangle_{c}}{6 \Delta}
$$


This approach results in the following tensor matrix,

$$
\mathbf{D}_{\text {cap }}=\left(\begin{array}{ccc}
0.24 & 0.01 & 0.01 \\
0.01 & 0.24 & 0.01 \\
0.01 & 0.01 & 2.30
\end{array}\right) \times 10^{-9} \mathrm{~m}^{2} / \mathrm{s} .
$$

The values correspond well with the results obtained by three $D-D$ maps with uncertainties.

By either using Equation (3.11) or Equation (3.12), the same value of $\mathrm{FA}=0.90$ can be derived. However, Equation (3.12) gives a smaller uncertainty of 0.3 (due to less calculation involved and less errors propagating), thus in the rest of the chapter, Equation (3.12) is used to calculate FA directly from the diffusion tensor in the laboratory coordinate system.

It should be noted that the bulk diffusion tensor could be constructed by using six single PFG experiments with different gradient directions, which may be more time-efficient than the proposed approach. However, individual single PFG experiments will return the same 1D distributions in a system as presented in Figure 5.4, thus they fail to resolve local anisotropic structure. However, by combining the observation of peak features in the correlation maps (i.e. qualitative analysis) and the derivation of bulk FA values (i.e. quantitative analysis) as presented in this thesis, it is possible to identify microscopical features of the system. This is similar to the idea of combing $\mu \mathrm{FA}$ with FA to better characterise crossing fibres as well as macroscopically isotropic system.

\subsection{Experimental}

As a widely used phantom in diffusion anisotropy experiments [174, 202], a carrot was measured as sample I. Sample II was a 6-week-old healthy mouse brain and sample III was an 8-week-old tumour-bearing mouse brain that had received an intracranial administration of GL261 glioma tumour cells 20 days prior to isolation [203]. Both brains were isolated immediately 
after the mice were euthanized and stored in IMDM medium (Invitrogen, Waltham, Massachusetts, USA) supplemented with 5\% fetal bovine serum (Sigma-Aldrich, St. Louis, Missouri, USA), and 2 mM glutamax, 100 U/ml penicillin, $100 \mathrm{mg} / \mathrm{ml}$ streptomycin, and $50 \mathrm{mM}$ 2-mercaptoethanol (all Invitrogen) at $4^{\circ} \mathrm{C}$. Both mice brains (with and without tumour) were measured in vitro.

Experiments were carried out on the Bruker Advance $400 \mathrm{MHz}$ NMR spectrometer (Figure 2.1) equipped with a Bruker Micro2.5 micro-imaging system with a maximum gradient of $1.45 \mathrm{~T} / \mathrm{m}$ and RF coil diameter of $25 \mathrm{~mm}$. All experiments were carried out at the ${ }^{1} \mathrm{H}$ resonance frequency of 399.14 $\mathrm{MHz}$ at ambient temperature of $20^{\circ} \mathrm{C}$. Experimental protocols were approved by the Victoria University Animal Ethics Committee.

DDCOSY experiments using the pulse sequence [133] in Figure 3.15 with the proposed three gradient orientation schemes shown in Figure 5.1 were applied for the three different biological samples. In order to achieve a pronounced influence of the tissue structure on the water diffusion, $\Delta$ was chosen to be $500 \mathrm{~ms}$ for carrot and $800 \mathrm{~ms}$ for brains. These parameters were set based on 1D diffusion measurements along different directions. The much longer observation time for brains was to eliminate the signal from the free fluid surrounding the tissues. $\delta$ was set to be $2 \mathrm{~ms}$ for all tissues. The amplitude of diffusion encoding gradient was increased linearly by 32 steps from -0.5 to $0.5 \mathrm{~T} / \mathrm{m}$ for the carrot, and from -0.732 to $0.732 \mathrm{~T} / \mathrm{m}$ for both brains respectively. The repetition time was $3 \mathrm{~s}$ and the number of scan was 4 , yielding the total acquisition time of 2 hours and 34 minutes for one sample.

DTI experiments were performed on the same equipment subsequently. The six independent gradient orientations were set the same with these indicated in Figure 5.1. The pulse sequence used in our experiments has been shown earlier in Figure 3.10. The $b$-values shown in Equation (3.8) were linearly increased from 0 to $800 \mathrm{~s} / \mathrm{mm}^{2}$ in 4 steps along every direction. This way sufficiently stables the obtained DTI results although the 
minimum required number of gradient directions was used. The resolution of DTI was $97.6 \mu \mathrm{m} \times 97.6 \mu \mathrm{m}$ in both directions, with a field of view of $25 \mathrm{~mm} \times 25 \mathrm{~mm}$ and a slice thickness of $1 \mathrm{~mm}$. The observation time and gradient duration of DTI were set to be the same as DDCOSY experiments. The repetition time for DTI was set to be $3 \mathrm{~s}$, the echo time was set to be 25 ms and the total imaging time for each sample was 3 hours.

\subsection{Results and Discussions}

\subsubsection{Plant tissue}

One slice obtained from the SE imaging sequence and the DDCOSY results of sample I are given in Figure 5.8. It reveals distinct behaviours depending on the different choices of gradient directions. The peak distributions of the correlation maps in Figure 5.8 (b) and (c) are similar, where the peaks with the largest intensities are located on the diagonal line. This indicates a rotationally symmetric structure in the $x y$-plane. Evaluating Figure $5.8(\mathrm{~b})$ to $(\mathrm{d})$ returns the largest diffusivity along the $z$-axis, suggesting global anisotropic diffusion preferably along $z$. In fact diffusion coefficients are distributed mainly off-diagonally in Figure 5.8 (d), highlighting again that this sample is globally anisotropic with little or no isotropic components (Figure 5.4).

The elements of diffusion tensor matrix were reconstructed from the DDCOSY experiments by using the same approach shown in Section 5.3.2, yielding

$$
\mathbf{D}_{\text {car }}=\left(\begin{array}{lll}
1.01 \pm 0.02 & 0.03 \pm 0.06 & 0.74 \pm 0.07 \\
0.03 \pm 0.06 & 1.02 \pm 0.02 & 0.74 \pm 0.05 \\
0.74 \pm 0.07 & 0.74 \pm 0.05 & 2.03 \pm 0.03
\end{array}\right) \times 10^{-9} \mathrm{~m}^{2} / \mathrm{s}
$$

By using Equation (3.12), FA $=0.72 \pm 0.02$ can be derived, showing a high degree of anisotropy of sample I. This may be caused by the elongated 


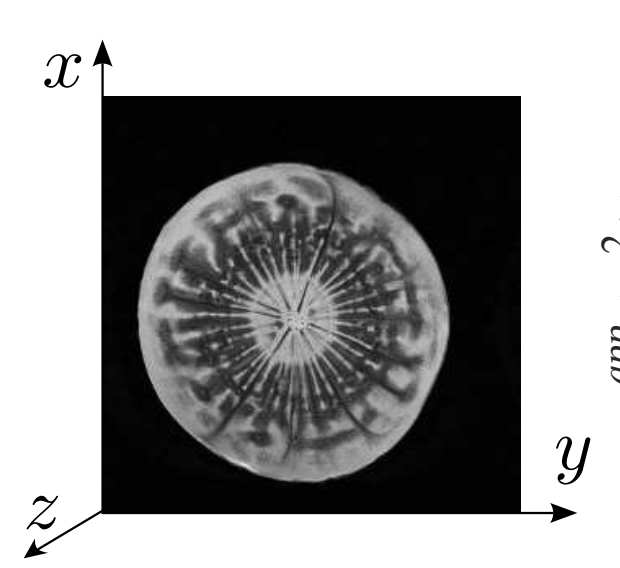

(a)

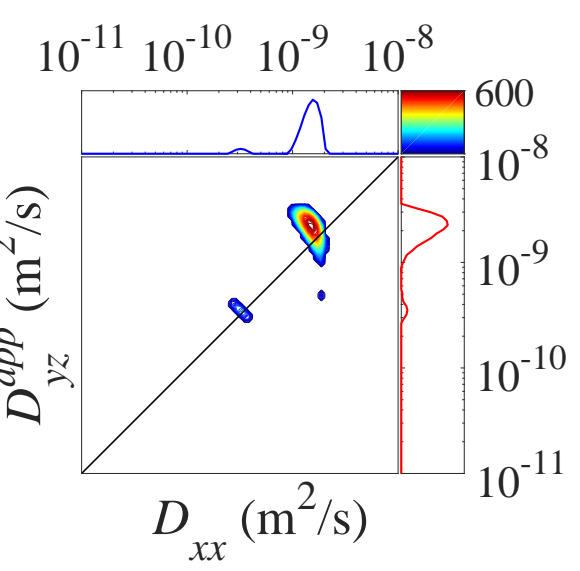

(b)

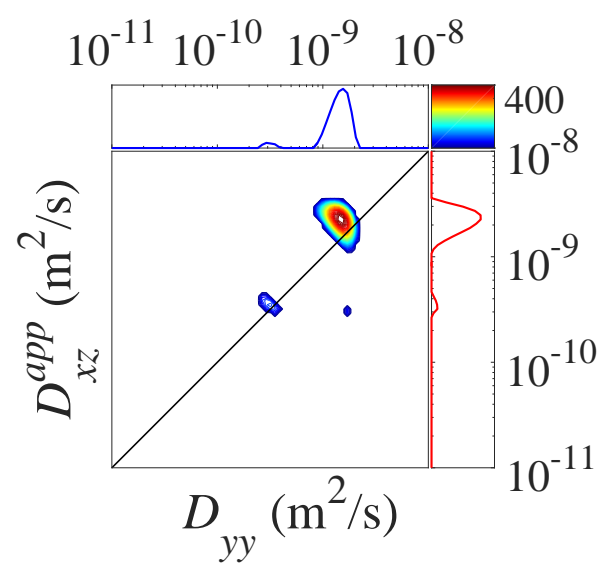

(c)

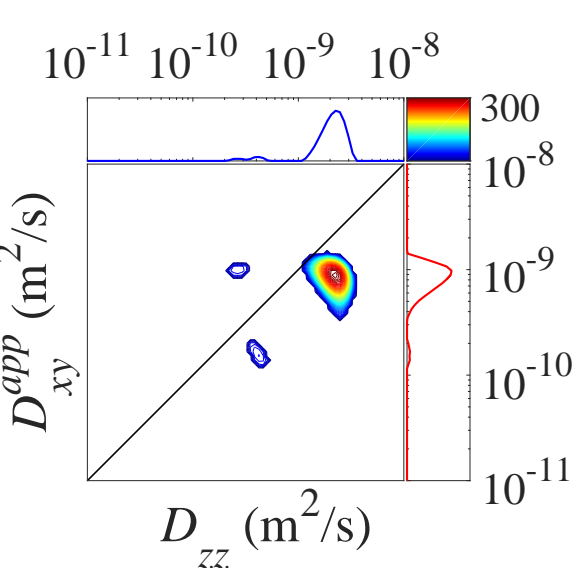

(d)

Figure 5.8: Cross-section image (a) of the carrot and the DDCOSY experiments using the proposed gradients combinations: (b) $x-y z$; (c) $y$ - $x z$; and (d) $z-x y$. 
shape of the plant cells such as the xylem cells (Figure 3.19) or water diffusing in the intercellular space. For comparison, the histogram of FA was determined from its DTI result, which is shown in Figure 5.9. FA is seen to be centred around 0.7 with an averaged value of 0.72 . This was in line with the value obtained from the proposed DDCOSY measurements ${ }^{3}$.

While the eigenvalues of the diffusion tensor are not used for calculating the FA, it is provided for a better comprehension of the anisotropic tissue structure:

$$
\begin{aligned}
& \lambda_{1}=0.38 \pm 0.15 \times 10^{-9} \mathrm{~m}^{2} / \mathrm{s}, \\
& \lambda_{2}=0.99 \pm 0.13 \times 10^{-9} \mathrm{~m}^{2} / \mathrm{s}, \\
& \lambda_{3}=2.69 \pm 0.15 \times 10^{-9} \mathrm{~m}^{2} / \mathrm{s} .
\end{aligned}
$$

Given that our observation time was $500 \mathrm{~ms}$, the length of cells could be $\sqrt{6 D \Delta}=\sqrt{6 \times 2.7 \times 10^{-9} \mathrm{~m}^{2} / \mathrm{s} \times 0.5 \mathrm{~s}}=90 \mu \mathrm{m}$, which was in the range of the mean cell size of carrot reported from $30 \mu \mathrm{m}$ to $100 \mu \mathrm{m}$ (as mentioned in Section 3.5.1). However, $D_{z z}$ and $\lambda_{3}$ appear to be above the value reported for free water diffusion at $20^{\circ} \mathrm{C}$ [80] (see Page 18). As suggested in Figure 2.7, this might be ascribed to the increasing temperature from the gradient and RF heating of the sample. Although different tissues would exhibit various degrees of heating, the variation was assumed to be uniform thus neglected when a bulk value is calculated. Therefore, temperature gradients would not affect the fractional anisotropy values.

To further validate the concept, experiments on other two different carrot samples were carried out. The $D-D$ maps showed similar patterns therefore is no longer present in the thesis.

\footnotetext{
${ }^{3}$ Although the FA map would reveal more detailed spatial information about the system, the purpose of performing DTI is for benchmarking the bulk result from DDCOSY, thus the FA map is omitted in this thesis
} 


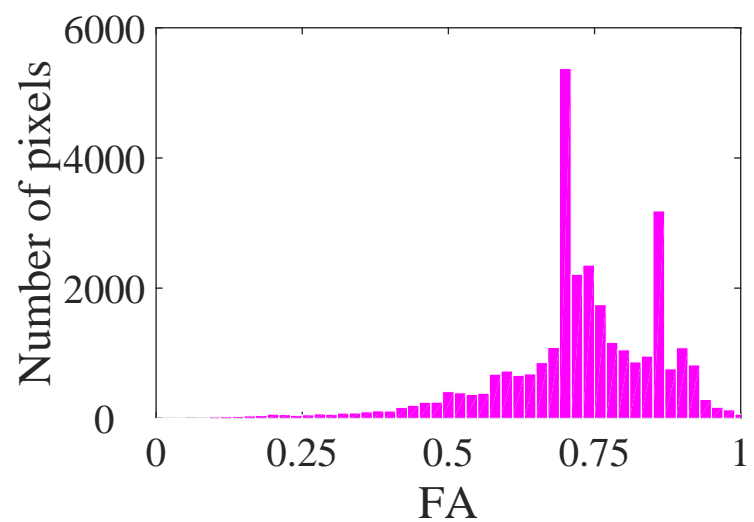

Figure 5.9: The histogram of FA values calculated from DTI results in the carrot.

\subsubsection{Animal tissues}

\section{Normal mouse brain}

The DDCOSY experiments of one animal tissue (mouse brain) without the injection of tumour (i.e. healthy) are shown in Figure 5.10. Given a long observation time we set, no separated isotropic peaks from the free fluid surrounding the brain tissues. The results in Figure 5.10 (c) and (d) are similar and the main diffusion coefficients in the first domain along the $y$ - and $z$-axis are constantly above $1 \times 10^{-10} \mathrm{~m}^{2} / \mathrm{s}$, whereas diffusion coefficients in Figure 5.10 (d) along the $x$-axis are mainly distributed below $1 \times 10^{-10} \mathrm{~m}^{2} / \mathrm{s}$. Off-diagonal peaks can be identified, eliciting that the healthy brain is microscopically anisotropic. Diagonal peaks can be observed in Figure 5.10 (b), while they disappear in (c) and (d). This suggests that the healthy brain is macroscopically anisotropic.

Again, by extracting the logarithmic mean values from the diffusion coefficient distributions in Figure 5.10 for each dimension, the diffusion 


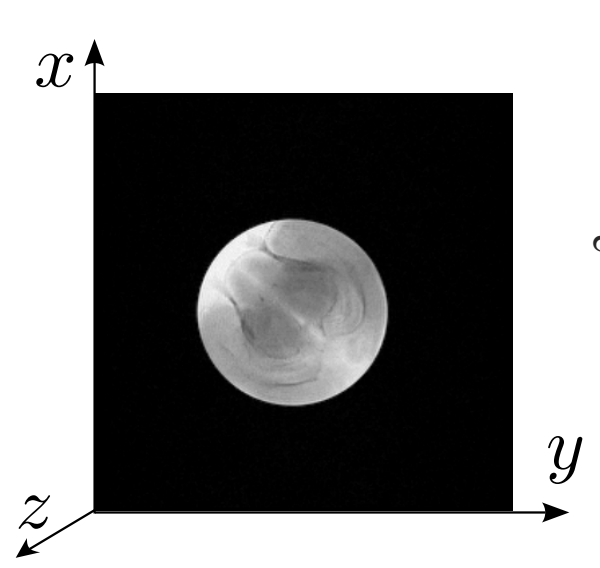

(a)

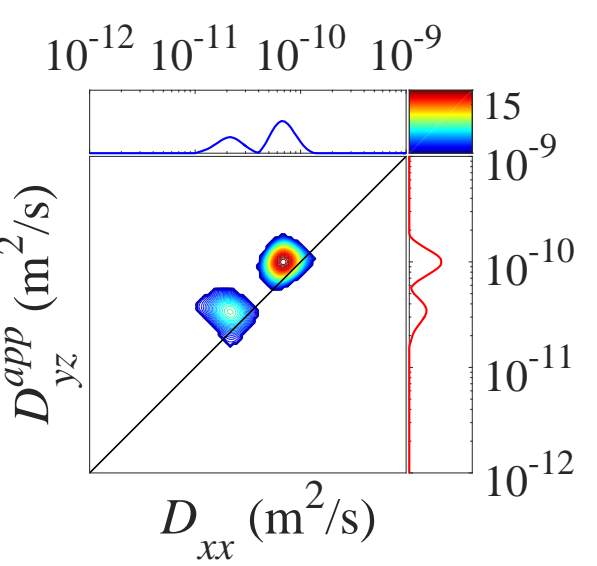

(b)

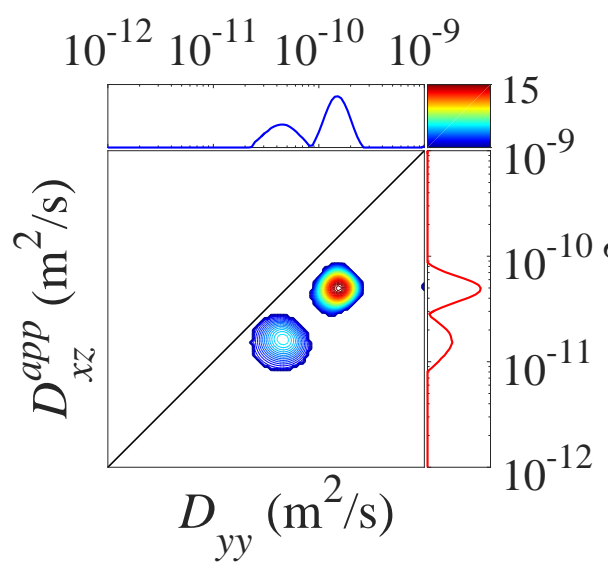

(c)

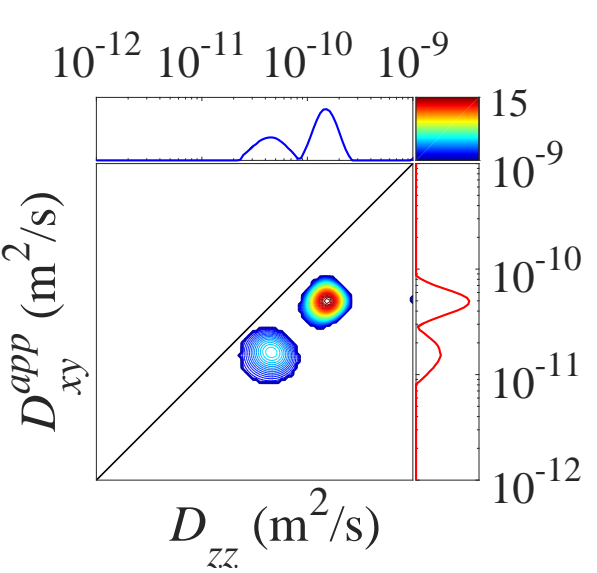

(d)

Figure 5.10: Cross-section image (a) of the healthy brain and the DDCOSY experiments using the proposed gradients combinations: (b) $x-y z$; (c) $y-x z$; and (d) $z-x y$. 
tensor matrix was calculated to be

$$
\mathbf{D}_{\text {nor }}=\left(\begin{array}{ccc}
0.45 \pm 0.02 & -0.31 \pm 0.02 & -0.38 \pm 0.05 \\
-0.31 \pm 0.02 & 0.95 \pm 0.05 & -0.28 \pm 0.06 \\
-0.38 \pm 0.05 & -0.28 \pm 0.06 & 0.95 \pm 0.04
\end{array}\right) \times 10^{-10} \mathrm{~m}^{2} / \mathrm{s}
$$

with the following eigenvalues:

$$
\begin{aligned}
& \lambda_{1}=0.06 \pm 0.01 \times 10^{-10} \mathrm{~m}^{2} / \mathrm{s}, \\
& \lambda_{2}=1.06 \pm 0.18 \times 10^{-10} \mathrm{~m}^{2} / \mathrm{s}, \\
& \lambda_{3}=1.24 \pm 0.15 \times 10^{-10} \mathrm{~m}^{2} / \mathrm{s},
\end{aligned}
$$

resulting in a FA $=0.68 \pm 0.03$. This is in agreement with values from ex-vivo measurements of mouse brain as reported in [204]. The histogram of FA values obtained from DTI of this sample is shown in Figure 5.11, which holds a mean value of 0.68 , supporting the result from the DDCOSY schemes. Anisotropy in mouse brain tissues may be attributable to the orientation of axonal tracks that affects water motion. Due to the various degrees of alignments, the environment of water motion in brain tissues is complex, neither pure isotropic nor anisotropic, thus resembling the fourth scenario as illustrated in Figure 5.6.

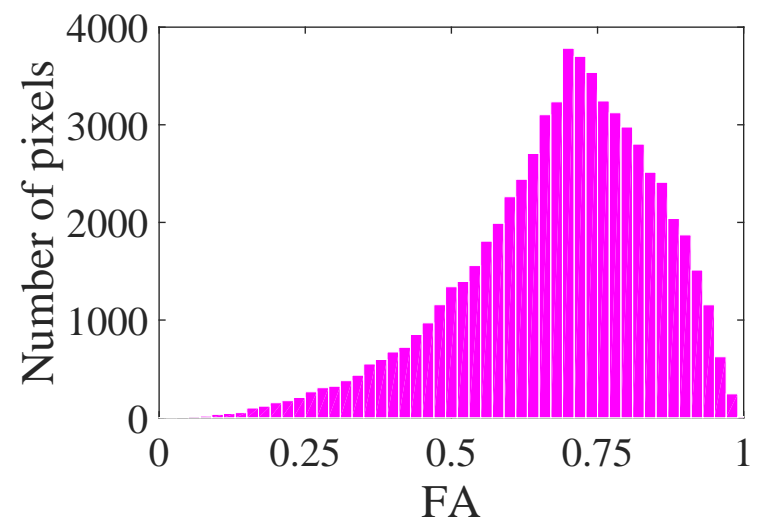

Figure 5.11: The histogram of FA values calculated from DTI results in the healthy brain. 
It may surprise that the mean FA value obtained for the healthy brain is similar to the mean FA value as obtained for carrots. However, this does not imply that the structures of these two tissues are similar or even identical. It only means that molecular diffusion is affected on a microscopic level by anisotropic obstructions in a similar way, thus the normalised diffusion ellipsoid obtained from DTI is similar.

\section{Tumour-bearing mouse brain}

In order to study the changes of the brain structures caused by a tumour, three DDCOSY experiments were performed on a mouse brain with an established tumour in the thalamus region. Figure 5.12 exhibits significant differences as compared to the results of the healthy brain shown in Figure 5.10. Firstly, all three maps contain peaks on the diagonals. Secondly, the positions of the dominant peaks in the 2D maps are nearly identical. Last but not least, all peaks in Figure 5.12 (c) and (d) are more symmetric to the diagonals. It is observed that the gradients applied to different axiscombination produce more similar DDCOSY distributions as compared to the healthy brain, indicating a substantial contribution of isotropic compartments locally. However, differences can still be spotted on the three 2D maps; for instance, only one diagonal peak is seen in Figure 5.12 (c), while two diagonal peaks are seen in Figure 5.12 (b) and (d). Off-diagonal peaks clearly prove that the system contains anisotropic components. It should be noted that the shape of peaks in $D-D$ maps of healthy brain is strikingly different from tumour-bearing brain. This is mainly ascribed to the different signal-to-noise ratio (SNR). The SNR of the healthy brain was found to be eight times smaller as compared to the other brain in our experiments ${ }^{4}$. Thus the 2D-ILT will automatically choose a larger smoothing factor, leading to more broadened peaks while detailed features will be lost. Detailed validation of this statement will be seen in Section 5.5.2.

\footnotetext{
${ }^{4}$ This may be caused by the fact that the size of sample II is much smaller than sample III, leading to a decreased water volume and detected signal in sample II
} 
However, the logarithmic mean values extracted from the peak positions will not change, which is critical in calculating the FA.

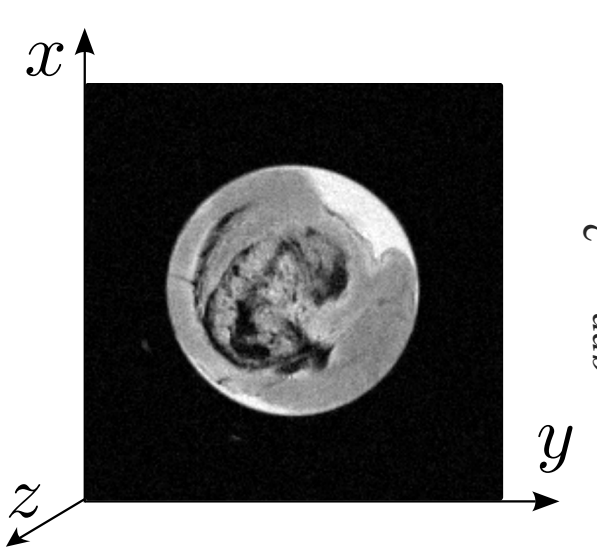

(a) sample III

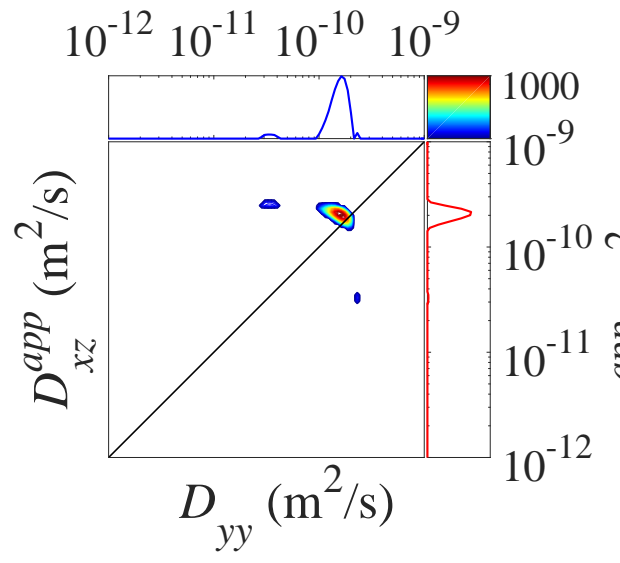

(c)

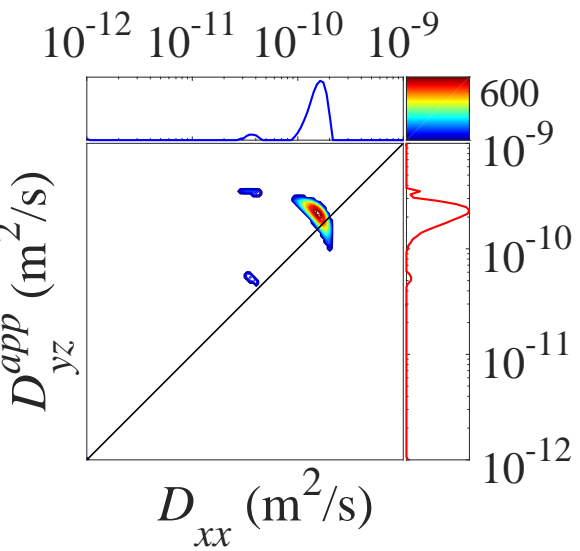

(b)

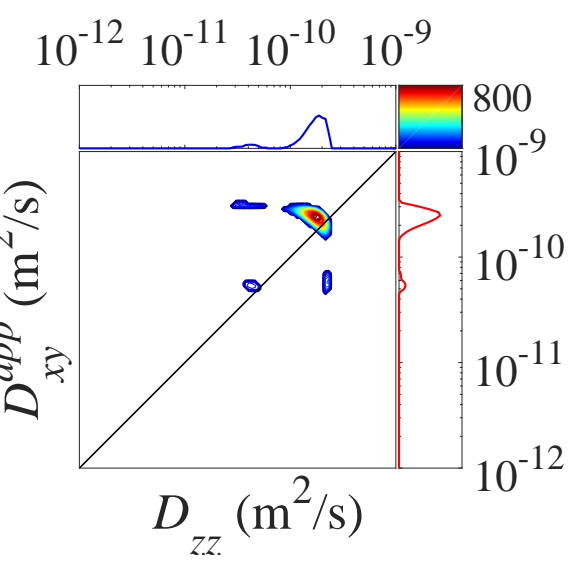

(d)

Figure 5.12: Cross-section image (a) of the tumour-bearing brain and the DDCOSY experiments using the proposed gradients combinations: (b) $x-y z$; (c) $y-x z$; and (d) $z-x y$.

Diffusion tensor elements were extracted subsequently from the three 
$D-D$ maps as provided by Figure 5.12,

$$
\mathbf{D}_{\text {tum }}=\left(\begin{array}{lll}
1.63 \pm 0.03 & 2.68 \pm 0.08 & 2.15 \pm 0.09 \\
2.68 \pm 0.08 & 1.63 \pm 0.06 & 2.11 \pm 0.08 \\
2.15 \pm 0.09 & 2.11 \pm 0.08 & 1.87 \pm 0.02
\end{array}\right) \times 10^{-10} \mathrm{~m}^{2} / \mathrm{s}
$$

with the eigenvalues of

$$
\begin{aligned}
& \lambda_{1}=0.60 \pm 0.01 \times 10^{-10} \mathrm{~m}^{2} / \mathrm{s}, \\
& \lambda_{2}=1.06 \pm 0.08 \times 10^{-10} \mathrm{~m}^{2} / \mathrm{s}, \\
& \lambda_{3}=2.95 \pm 0.18 \times 10^{-10} \mathrm{~m}^{2} / \mathrm{s},
\end{aligned}
$$

yielding $\mathrm{FA}=0.60 \pm 0.03$, which was smaller than the mean FA values in the healthy brain tissue. This may be attributed to more irregular orientation of the fibres growing inside the cancerous tissues [201]. The histogram of FA in the tumour-bearing brain is shown in Figure 5.13, and a mean value of 0.59 was found. This was again in accord with the DDCOSY calculations.

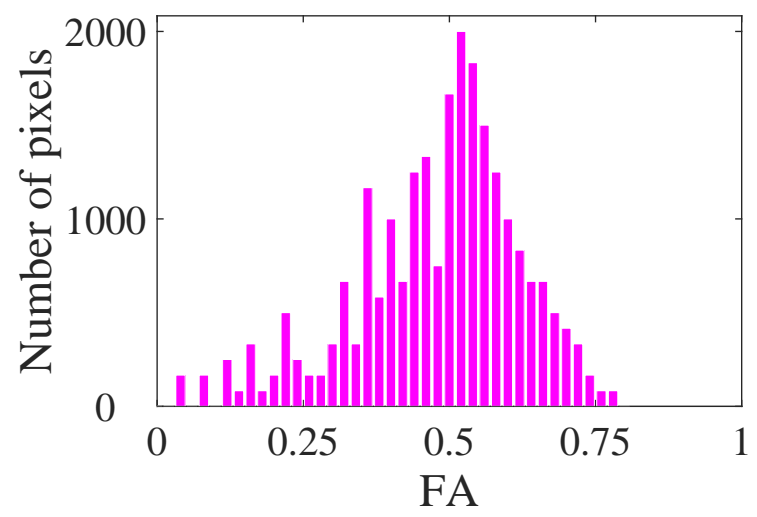

Figure 5.13: The histogram of FA values calculated from DTI results in the tumourbearing brain.

Surprisingly, only a $10 \%$ decrease of sample-averaged FA of the tumourbearing brain was found as compared to the healthy counterpart which may be due to the partial volume effect. Therefore, further investigation 
was exploited based on the DTI experiments. The tumour area was defined in the DTI results and the pixels that the tumour occupied were counted in order to calculate the volume ratio. The corresponding FA histogram of this tumour region is shown in Figure 5.14 with a mean value of 0.47 . This is consistent with FA values in glioma as found in the literature ranging from 0.1 to 0.7 , due to different observation times of DTI protocols and variable grades of glioma used in the independent experiments [205, 206]. By further processing the DTI data, the tumour was found to take up $40 \%$ of the total brain volume. If a simple bi-compartment model was assumed, the mean FA value of tumour-bearing brain was $0.47 \times 0.4+0.68 \times 0.6=0.59$, which again validated the mean values of FA obtained by DDCOSY.

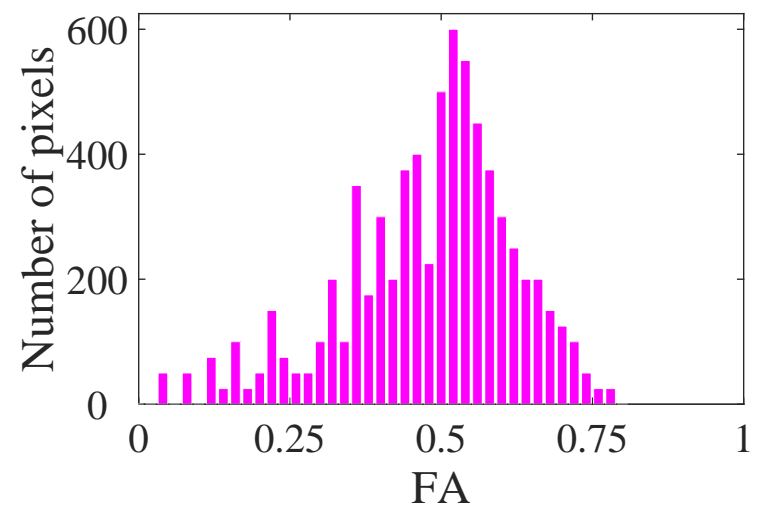

Figure 5.14: The histogram of FA values of the isolated tumour part from the tumour-bearing brain.

While the decrease of FA in the tumour-bearing brain is significant but small, it is possible that our approach may be insensitive to smaller tumours as compared to the total tissue volume without supplementary means of volume localisation. It is envisioned that this could be achieved for instance by combining low-field NMR concepts (which conveniently limit the detected sample volume by the "sweet spot") [207] with our scheme. Alternatively, coarse grain imaging methods or appropriate 3D slice selection schemes could be another means of limiting the sample volume under 
investigation, thus controlling the effective tumour-to-total volume ratio. Nevertheless, there are observable differences between the two tissues types (healthy and tumour) in the patterns of $D-D$ maps which may assist in discriminating them. These isotropic patterns may be attributed to the destruction of aligned fibres during the tumour growth [208], resulting in a smaller overall fractional anisotropy.

\section{The SNR influence on the peak shape of 2D $D-D$ map}

In order to understand the difference of peak shapes in Figure 5.10 and Figure 5.12, another healthy mouse brain (sample IV) was prepared which had a similar size as compared to sample III and contained same volume of water, implying that the signal intensities should be at the same level. Sample IV was measured using the same parameters of DDCOSY experiments as used in sample II and III. The MRI slice and the $D-D$ maps of sample IV are shown in Figure 5.15. The SNR of sample IV was at the same level of sample III. It is observable that peak features (but not positions) are also similar in Figure 5.12 (b) and Figure 5.15 (b).

By extracting the logarithmic mean values from the diffusion coefficient distributions in Figure 5.15 for each dimension, the diffusion tensor matrix was calculated to be

$$
\mathbf{D}_{\text {nor }}=\left(\begin{array}{ccc}
1.45 \pm 0.02 & -0.80 \pm 0.12 & 0.97 \pm 0.09 \\
-0.80 \pm 0.12 & 1.45 \pm 0.02 & 0.47 \pm 0.09 \\
0.97 \pm 0.08 & 0.47 \pm 0.08 & 2.50 \pm 0.06
\end{array}\right) \times 10^{-10} \mathrm{~m}^{2} / \mathrm{s}
$$

with the following eigenvalues:

$$
\begin{aligned}
& \lambda_{1}=0.18 \pm 0.22 \times 10^{-10} \mathrm{~m}^{2} / \mathrm{s} \\
& \lambda_{2}=2.13 \pm 0.23 \times 10^{-10} \mathrm{~m}^{2} / \mathrm{s} \\
& \lambda_{3}=3.08 \pm 0.24 \times 10^{-10} \mathrm{~m}^{2} / \mathrm{s}
\end{aligned}
$$

which resulted in a FA $=0.68 \pm 0.02$. It is found that the value of FA was 


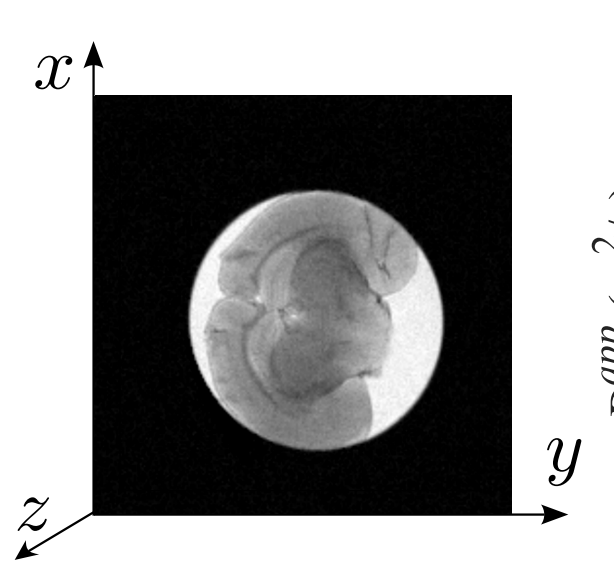

(a)

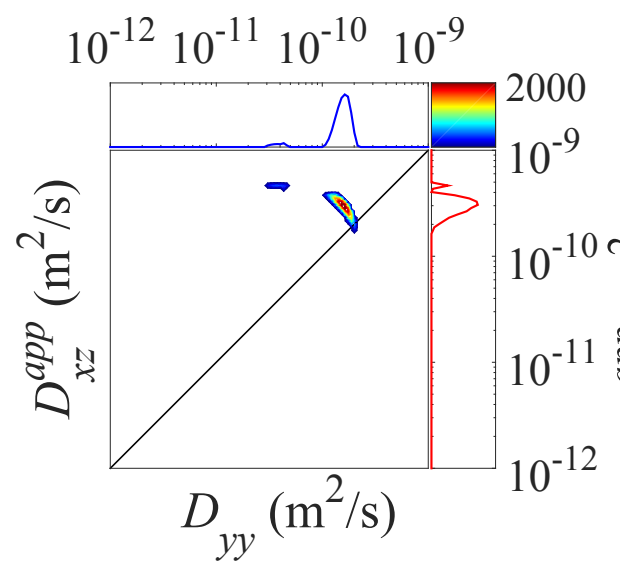

(c)

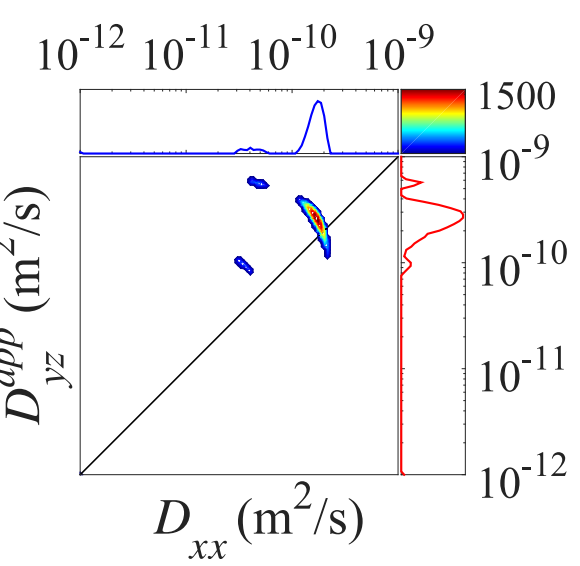

(b)

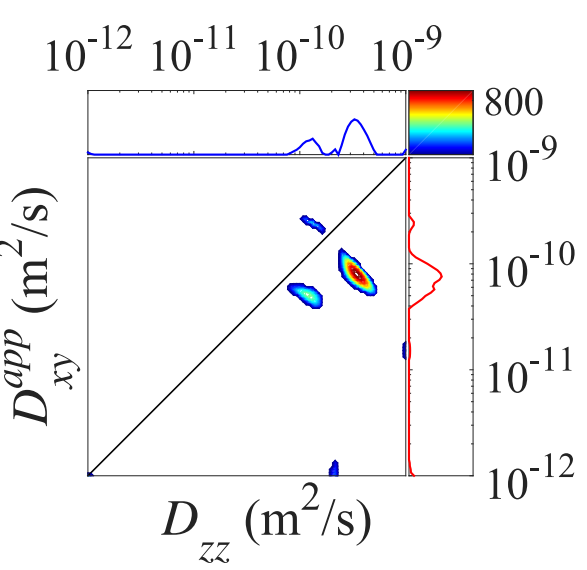

(d)

Figure 5.15: Cross-section image (a) of another healthy brain and the DDCOSY experiments using the proposed gradients combinations: (b) $x-y z$; (c) $y-x z$; and (d) $z-x y$. It can be seen from the image that is healthy brain had similar size to sample III. 
identical to that was calculated in sample II, however, a slightly smaller uncertainty presented in sample IV. One may notice a 3-fold increase in the $\lambda$-values of sample IV as compared to the values of sample II, which is attributed to the calculation of log mean value on spectra with different width. The broader distribution will result in more deviation of the peak value. What need to be noted here is that the degree of the deviation were the same for three eigenvalues, thus FA of the two samples were the same.

The signal decay of sample IV obtained from the $x$-yz gradient scheme is shown in Figure 5.16 (a). By manually superposing a Gaussian noise, the signal decay became Figure 5.16 (b), where a similar SNR level to sample II was obtained. The resulting $D-D$ map after 2D-ILT is shown in Figure 5.17. It is observed that the shape of the peak in Figure 5.17 is akin to that in Figure 5.10 (b), but differs now from Figure 5.15 (b). The peak become spread, leading to a limited resolution. However, the position of the main peak in Figure 5.15 (b) and Figure 5.17 remains the same, which poses very small influence to the calculation of the logarithmic mean values of the diffusion tensor.

It may be argued that when SNR is below a certain limit, off-diagonal peaks may not be resolved in the $D$ - $D$ map, therefore an anisotropic system might appear isotropic in the 2D map. However, it should be kept in mind that although the peaks may not be separated, the position of the main peak will still be off-diagonal (because the SNR level does not influence the position of the main peak). The quantitative method as presented in this thesis will allow obtaining a non-zero mean FA values, and subsequent identifying the anisotropic system. Therefore, the combination of the qualitative 2D map and the quantitative measure is of significant importance to characterise the micro-structure of a system.

One may find parameters such as the restriction size and tortuosity can reveal intrinsic properties of microstructure [84, 209]. However, the access to these parameters either involves more mathematical fitting procedures or more experiments than FA. For instance, in order to obtain the tortuosity, 
diffusion coefficients need to be measured at different observation times, leading to a overall long time measurement. Therefore, only FA was considered in this thesis, and the current protocol can be extended to obtain such information by rearranging the sigal decay equation and including more experimental data.

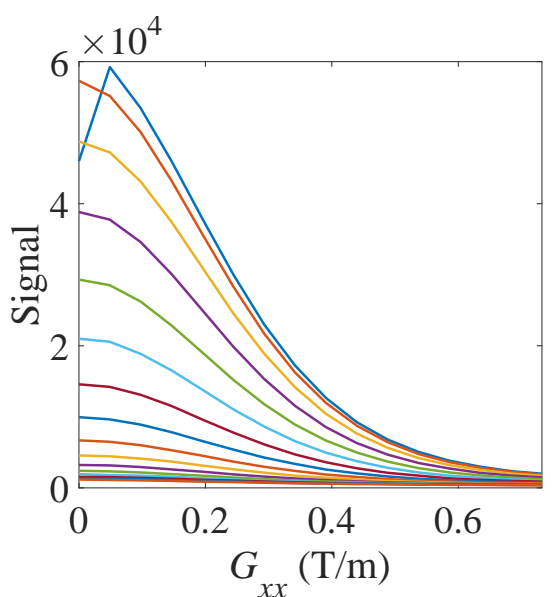

(a)

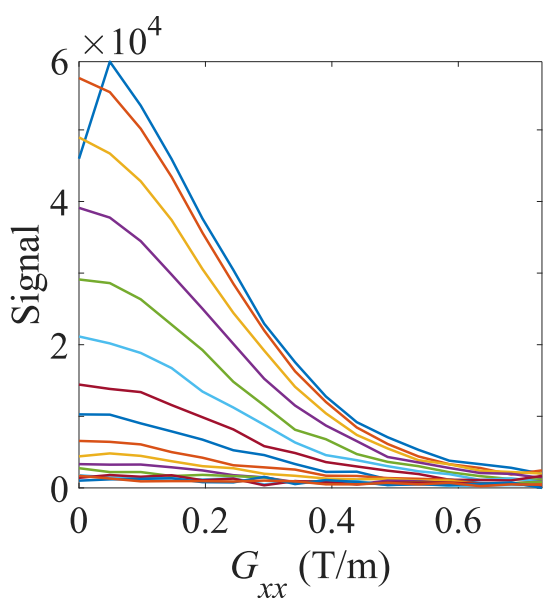

(b)

Figure 5.16: Signal decays before (a) and after (b) addition of Gaussian noise for various values of $G_{y z}$

\subsection{Conclusions}

Random walk simulations of four scenarios were implemented by applying the conventional DDCOSY strategy. The comparison of different scenarios supported that the 2D $D-D$ map can be used to distinguish the microscopic environments of tissues. Further simulation on aligned fibres validated the concepts of obtaining mean FA values by employing DDCOSY in combination with three particularly chosen gradient direction schemes.

As a proof of concept, the experiment on a carrot tissue demonstrated that our approach is capable of obtaining FA which compares very well to 


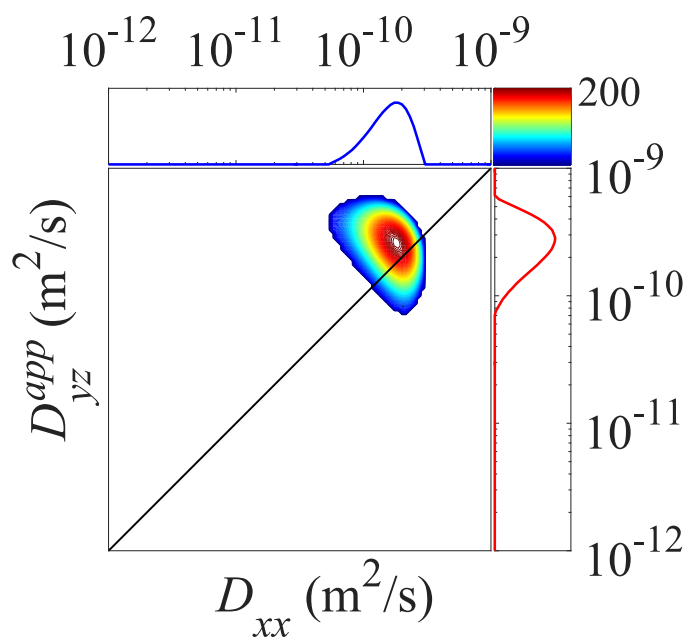

Figure 5.17: The $D-D$ map after superposing the Gaussian noise using the gradient combination of $x-y z$. The $D-D$ map before adding noise is shown in Figure 5.15 (b).

the averaged value obtained from the imaging method. In addition, the experiments conducted with two brain tissues revealed that the healthy brain has a higher FA, also leading to larger differences in the patterns of the three $D-D$ maps. On the contrary, the tumour-bearing brain has a reduced FA accompanied by more isotropic patterns in the $D-D$ maps. Moreover, these patterns of diffusion coefficients along all diffusion directions in the tumour-bearing brain were essentially identical, thus providing a contrast to the pattern obtained from the healthy brain. 


\section{Chapter 6}

\section{Quantitative Characterisation of Breast Tissue - A Field Study}

MRI has been developed as a routine approach in the clinical study of detecting cancers. Known as magnetic resonance mammography (MRM), it has emerged as a promising modality for detection, diagnosis, and staging of breast cancer. The purpose of this chapter is to evaluate the variability of DWI quantifications (apparent diffusion coefficients, perfusion factor, diffusivity and kurtosis) with different regions of interests, and introduce a threshold isocontouring strategy in order to increase intra-readers repeatability. 


\subsection{Introduction}

Breast cancer is the leading disease in women [210]. According to the cancer statistics reported in 2013, one in eight women in the United State have a breast cancer [211]; About 15,321 people in New Zealand died in 2012 because of various cancers, of which breast cancer accounts for $40 \%$ [212]. The latency period for breast cancers usually takes five to eight years allowing for early detection, which is essential for patient survival.

Breast cancers can be detected in a number of ways, including the presence of certain signs and symptoms, screening tests and medical imaging. Breast imaging methods include X-ray mammography, ultrasonography, MRI and PET. While mammography represents the "golden standard" for breast cancer screening since 1969, it still has certain limitations, including false-positive results and radiation exposure. Moreover, it shows low performance in finding cancers in dense tissues [213]. MRI has been proven to be more sensitive with the aid of contrast agents as compared to X-ray mammography [214]. The wash-out pattern in the time-signal intensity curve shown in Figure 3.9 is a strong indication for malignancy. But issues such as low specificity, long examination time and high costs in the DCE-MRI were reported [215]. However, these limitations were overcome by introducing the DWI technique into the field of breast imaging [216]. It allows measuring the mobility of water molecules which can be quantified by the values of apparent diffusion coefficients (ADC) [31]. Other quantifications, such as perfusion factor $(f)$, diffusivity $(D)$ and Kurtosis $(K)$ [115, 217-221], also describe different behaviours of water mobility in voxels of tissues. As malignant breast lesions are commonly characterised by densely packed cells, water mobility inside will be considerably restricted, yielding small mean ADC but large $K$ values [217, 222].

However, the structural composition of breast tissues is heterogeneous, which contains fatty, fibrous, glandular and lobular components, leading to the large variation of the ADC values with different tissues. Furthermore, 
regions with different volumes and volumetric ratios of these tissues affect the obtained ADC values [39, 223]. Even if a uniform region is selected for interest, the representative ADC values can be different due to averaging methods (e.g. mean, median) used in various studies as summarised by Kim and co-workers [224]. This emphasises that a correct definition of ROI and a proper measuring method are essential to separate tumour types more effectively and accurately.

However, the classification of breast lesions based on DW images is usually difficult because of the undistinguishable boundaries. Similar difficulties were found in PET images [225], where the intensity represents a relative concentration of radiotracers in any given pixel after injection. In order to delineate tumour boundaries in PET images, a "threshold isocontouring" procedure is often used [226] which selects pixels with an intensity higher than a certain threshold. These pixels are subsequently defined as the tumour region. The similarity between breast DWI and PET suggests utilising an analogous isocontouring procedure in DW images as will be presented in this chapter, thus providing better tumour categorisation in DW images.

In this chapter, the dependencies of diffusion-related quantifications on the size of ROIs are investigated, followed by the evaluation of the applicability to use the threshold isocontouring strategy in the available DWI data. In addition, different measuring methods are compared and the impact of threshold isocontouring on the diagnostic accuracy is also addressed.

\subsection{Methods}

\subsubsection{Participants and MR Imaging}

The clinical trials leading to this pilot study thesis work were carried out in the German Cancer Research Centre (Deutsches Krebsforschungszen- 
trum, Heidelberg, Germany). The retrospective analysis was approved by an institutional and governmental ethical review board. Written informed consent was obtained. The pilot study presented in this thesis included data from 23 female participants with suspicious findings on screening X-ray mammograms (BIRADS 4-5) (December 2014 to February 2015).

Breast MRI examinations were performed prior to the biopsy using a clinical 1.5 T MRI scanner (Siemens) with an 18-channel breast coil in Mannheim radiology centre (Radiologiezentrum Mannheim, Mannheim, Germany). All participants were placed in a prone position with the breasts slightly fixed in the dedicated breast coil using foamed material. DWI data was acquired with $b$-values of $0,0.1,0.75$ and $1.5 \times 10^{9} \mathrm{~s} / \mathrm{m}^{2}$ using a SE-EPI sequence with spectral attenuated inversion recovery (SPAIR) fat saturation [227] $\left(T_{\mathrm{R}} / T_{\mathrm{E}}=1.43 \mathrm{~s} / 0.08 \mathrm{~s}\right)$. The FOV was $0.48 \mathrm{~m} \times 0.24 \mathrm{~m} \times$ $0.3 \mathrm{~m}$, the matrix size was $192 \times 96 \times 50$, and the bandwidth was $870 \mathrm{~Hz} /$ pixel.

All included participants underwent ultrasound- or X-ray-guided breast biopsy in concordance to the regular mammography screening procedures. All biopsies were performed after the MRI examination, and the histopathological diagnosis served as a standard of reference for final validation of the quantitative analysis.

\subsubsection{ROI determination}

For each patient data, four ROIs were used to test the effect of varying ROI sizes and positions on ADC, $f, D$ and $K$ differences. For simplicity, all ROIs in this study were rectangularly shaped. The four ROIs were determined based on DCE-MR images and transferred to DW images as follows:

- $\mathrm{ROI}_{1}$ : defined by a radiologist with 2-year experience;

- $\mathrm{ROI}_{2}$ : obtained by expanding 2 pixels of the $\mathrm{ROI}_{1}$ in $x$ and $y$ dimensions; 
- $\mathrm{ROI}_{3}$ : obtained by shifting 2 pixels of the $\mathrm{ROI}_{1}$ in $x$ and $y$ directions;

- $\mathrm{ROI}_{4}$ : drawn by another independent radiologist with 10-year experience.

\subsubsection{Threshold isocontouring}

After determining the ROI $\left(\mathrm{ROI}_{1,2,3 \text { or } 4}\right)$ in the DW image with $b=$ $1.5 \times 10^{9} \mathrm{~s} / \mathrm{m}^{2}$ (b1500 image), a threshold $(\epsilon)$ relative to the maximal signal intensity ( $\left.S_{\max }\right)$ in the ROI was used to select a number of pixels (Npixels) for subsequent analysis. The selection criterion was defined as the signal intensity of the pixel $\left(S_{i}\right)$ above the threshold level, i.e.

$$
S_{i} \geq \epsilon \cdot S_{\max },
$$

where $i$ is the pixel number. $\epsilon=0$ indicates that all pixels in the ROI were selected whereas $\epsilon=1$ means only the pixels with signal intensity equal to $S_{\max }$ were chosen. This procedure was repeated for each type of ROI as introduced in Section 6.2.2.

In addition, in order to find the optimal thresholds for these four quantifications, 20 threshold values linearly distributed in the range of $[0,1]$ were applied to the four types of ROI in this study.

\subsubsection{Averaging measurements}

Once the pixels were selected by Equation (6.1), three averaging algorithms were applied, which will be named as "Averaged Signal", "Pixelwise Mean" and "Pixelwise Median" in this chapter. "Averaged Signal" means pixel signals inside the threshold-isocontourred ROI were averaged prior to the calculation of ADC, $f, D$ and $K$ values, while "Pixelwise Mean" and "Pixelwise Median" indicates that the ADC, $f, D$ and $K$ values of each pixel was calculated individually. "Pixelwise Mean" picks the mean value, 
while "Pixelwise Median" chose the median value of the selected pixels as the representative for each diffusion parameter.

\subsubsection{Mathematical models}

The calculations of the four quantifications were performed by fitting the signal intensities with various $b$-values. The fitting models were IVIM (Equation (3.5)) and diffusion kurtosis models (Equation (3.15)) as introduced in Section 3.2.2 [220, 221].

Regarding to the use of the IVIM model, as $\mathrm{ADC}_{p}$ is much faster than $\mathrm{ADC}$ [116], ADC was fitted firstly with signals acquired at $b$-value larger than $0 \mathrm{~s} / \mathrm{m}^{2}$, where $S(0) \cdot f \cdot\left(-b \cdot \mathrm{ADC}_{p}\right)$ is considered to be $0 . f$ is calculated afterwards by substituting the fitted $\mathrm{ADC}$ and $S(0)$ and values into Equation (3.5).

The diffusion kurtosis model essentially describes a mono-exponential decay, which may lead to an erroneous fit if blood vessels are present in the breast tissue. Therefore, when using diffusion kurtosis model to fit the acquired NMR data, $\mathrm{b}_{0}\left(b=0 \mathrm{~s} / \mathrm{m}^{2}\right)$ data point was excluded to minimise the perfusion effects.

\subsubsection{Statistical analysis}

After processing all included patients' datasets as described in Section 6.2.2 - 6.2.5, a series of statistical analysis was performed by correlating the quantification values with the histopathological information.

In order to compare the diagnostic accuracies of different quantifications using three averaging methods, the receiver operating characteristic (ROC) curves were analysed. The ROC curves are $2 \mathrm{D}$ graphical plots, with vertical axis being the true positive rate (i.e. sensitivity) and the horizontal axis being the false positive rate (i.e. 1-specificity) respectively [228]. Each data point in a ROC curve is obtained by evaluating the probabilities of four classifications (true positive, false positive, true negative and false negative) 
at a certain discriminative (i.e. cut-off) value ${ }^{1}$. A single quantitative index of the diagnostic accuracy can be reflected by computing the area under a ROC curve, which is commonly referred to as the area under curve (AUC) [228]. Larger AUC values indicate higher diagnostic accuracy of the studied method.

Differences between measured values for benign and malignant lesions were evaluated using the two-sample Student's $t$-test ${ }^{2}[229]$ after normality testing using the Shapiro-Wilk test ${ }^{3}$ [230]. The two-sample Student's $t$-test is one type of statistical hypothesis tests. It is often used to determine whether two datasets (e.g. ADC values of benign and malignant lesions) are significantly different from each other. The normality test is frequently used to determine whether a dataset follows a normal distribution, which is the prerequisite to perform the Student's $t$-test. A $p$-value of less than 0.05 was considered significant. All analyses in Section 6.2.2 - 6.2.6 were implemented by using MATLAB R2015b software (Mathworks, Cambridge, UK).

\subsection{Results}

\subsubsection{Lesion information and representative images}

Of 23 subjects imaged for this study, 3 were excluded due to the invisibility of lesions on the b1500 images. Among the DWI-detectable lesions, 7 were benign, including cyst $(n=2)$, fibroadenoma $(n=4)$, and chronic inflammation $(n=1)$. Whilst 13 lesions were malignant, including invasive

\footnotetext{
${ }^{1}$ The detailed implementation can be found in http://www.mathworks.com/ help/stats/perfcurve.html.

${ }^{2}$ The detailed implementation of the two-sample Student's $t$-test can be found in http://www. mathworks.com/help/stats/ttest2.html.

${ }^{3}$ Shapiro-Wilk test is one type of the normality test, suitable for the sample size between 3 and 5000. The detailed implementation of the Shapiro-Wilk test can be found in the website of http://www. mathworks.com/matlabcentral/fileexchange/ 13964-shapiro-wilk-and-shapiro-francia-normality-tests/content/ swtest.m.
} 
ductal carcinoma (IDC, $n=11$ ), invasive lobular carcinoma (ILC, $n=1$ ) and ductal carcinoma in situ (DCIS, $n=1$ ). The mean age of the subjects was $59.4( \pm 10)$ years old. Figure 6.1 shows representative images of benign and malignant lesions. Figure 6.1 (a) is from a 50 years old patient with a fibroadenoma, while Figure 6.1 (b) is from a 54 years old patient with an IDC. The images in the first row were obtained from DCE-T1WI, while the second row shows b1500 images. Both lesions were visible and shown high intensities in DCE $T_{1}$-weighted and DW images, indicating that it is impossible to identify the pathological differences only using the images, and that further quantitative analysis needs to be performed in order to distinguish them.

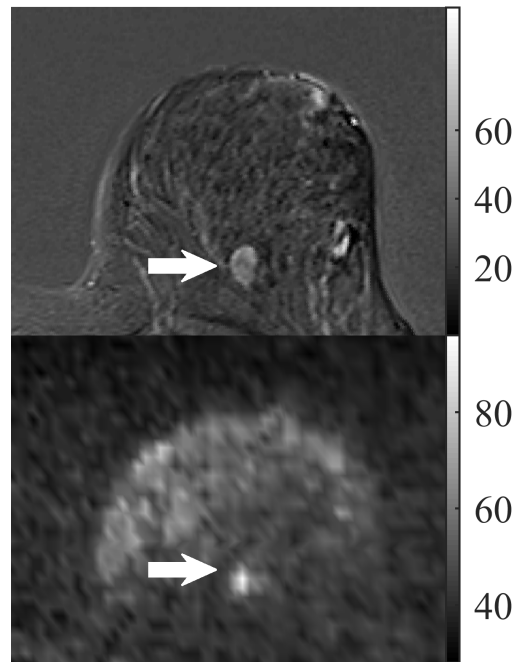

(a)

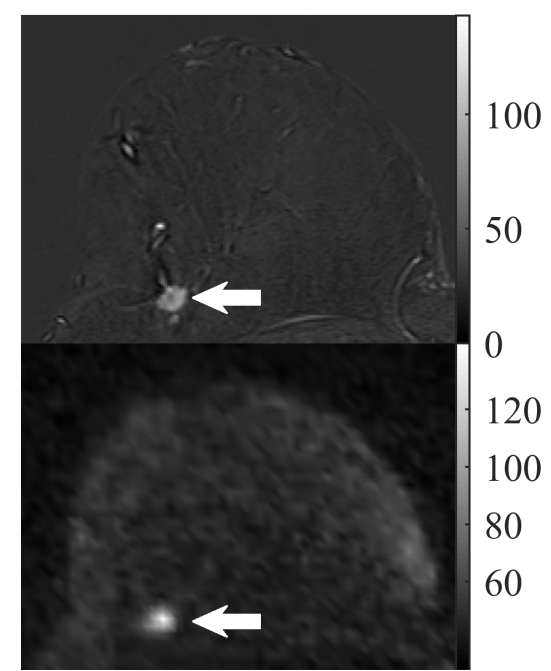

(b)

Figure 6.1: DCE-T1WI (upper panel) and DWI with $b$ of $1500 \mathrm{~s} / \mathrm{mm}^{2}$ (lower panel) of (a) a 50 year-old female patient with fibroadenoma and (b) a 54 year-old female patient with IDC. 


\subsubsection{Dependency of quantifications on thresholds, ROI types and averaging measurements}

The ADC, $f, D$ and $K$ values were calculated in the four types of ROI by using the three averaging measurements according to Section 6.2.2 - 6.2.5. The quantitative analyses were repeated at various threshold values, and the results of the two representative subjects were shown in Figure 6.2 and Figure 6.3 respectively. Dependencies and variations of Npixels, ADC, $f, D$ and $K$ values against different relative thresholds, ROI types and averaging measurements are seen in both cases. The ADC, $f, D$ and $K$ values for the four ROIs were diverse at the beginning, but converged at specific thresholds independent of individual measurements. For instance, as can be seen in Figure 6.2 (a), initial ADC values from "Averaged Signal" for $\mathrm{ROI}_{1}, \mathrm{ROI}_{2}, \mathrm{ROI}_{3}$ and $\mathrm{ROI}_{4}$ were $1.15,0.94,1.07$ and $1.35 \times 10^{-9} \mathrm{~m}^{2} / \mathrm{s}$, respectively. They all approached to be $1.33 \times 10^{-9} \mathrm{~m}^{2} / \mathrm{s}$ when the threshold was larger than 0.55 . All three averaging measurements returned the ADC, $f$, and $D$ values with larger deviations at smaller thresholds, but the same values when the thresholds reached to 1 . However, the relative change of $K$ values in $\mathrm{ROI}_{2}$ when varying the thresholds from 0 to 1 was found to be the largest. The four quantifications finally returned the same value for each parameter when threshold arrived at 0.85 . In this case, 3 pixels were selected for calculation. The initial values in $\mathrm{ROI}_{4}$, the size of which was the smallest, were found to be the closest to the converged value.

Figure 6.3 follows the same tendency. The only slight difference was that the threshold for the ADC, $f, D$ and $K$ values to converge was at 0.75 . By comparing the converged values of the two representative subjects, the converged ADC and $D$ values in Figure 6.3 were found to be smaller as compared to the values in Figure 6.2, while $f$ and $K$ values were larger in the malignant lesions.

The observer differences $\left(\delta_{\mathrm{ADC}}, \delta_{f}, \delta_{D}\right.$ and $\left.\delta_{K}\right)$ averaged on all included patients datasets were shown in Figure 6.4 when comparing $\mathrm{ROI}_{1}$ and 

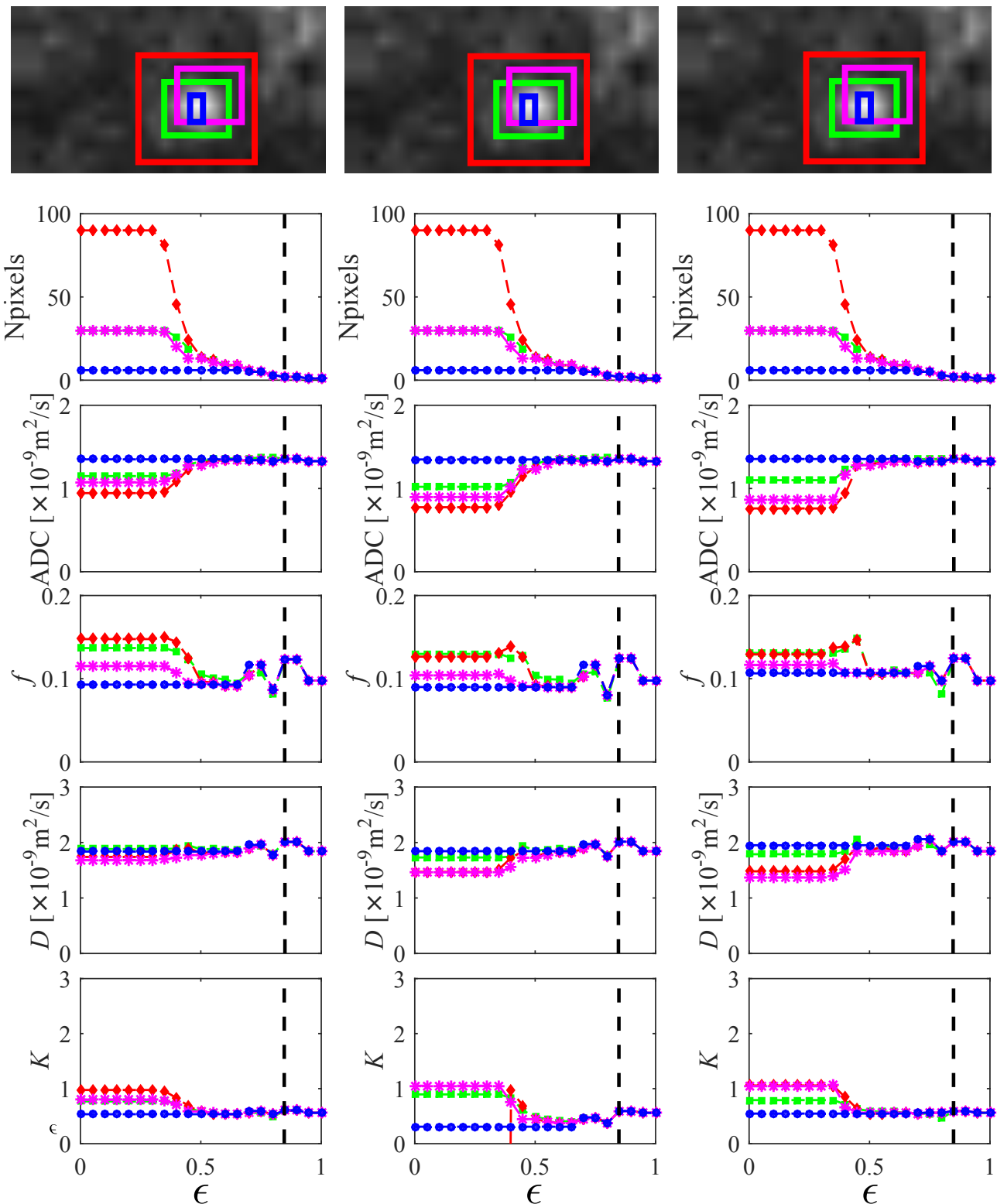

(a)

(b)

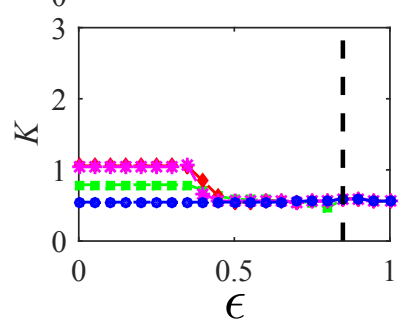

(c)

Figure 6.2: Data evolution for Npixels, $\mathrm{ADC}, f, D$ and $K$ values of the lesion in Figure 6.1 (a) as a function of thresholds based on $\mathrm{ROI}_{1}(\square), \mathrm{ROI}_{2}(\diamond), \mathrm{ROI}_{3}(*)$ and $\mathrm{ROI}_{4}(\bullet)$ on the b1500 image, using three different measurements: (a) Averaging signal; (b) Pixelwise Mean; (c) Pixelwise Median. Vertical black dash lines indicate $\epsilon=0.85$. 

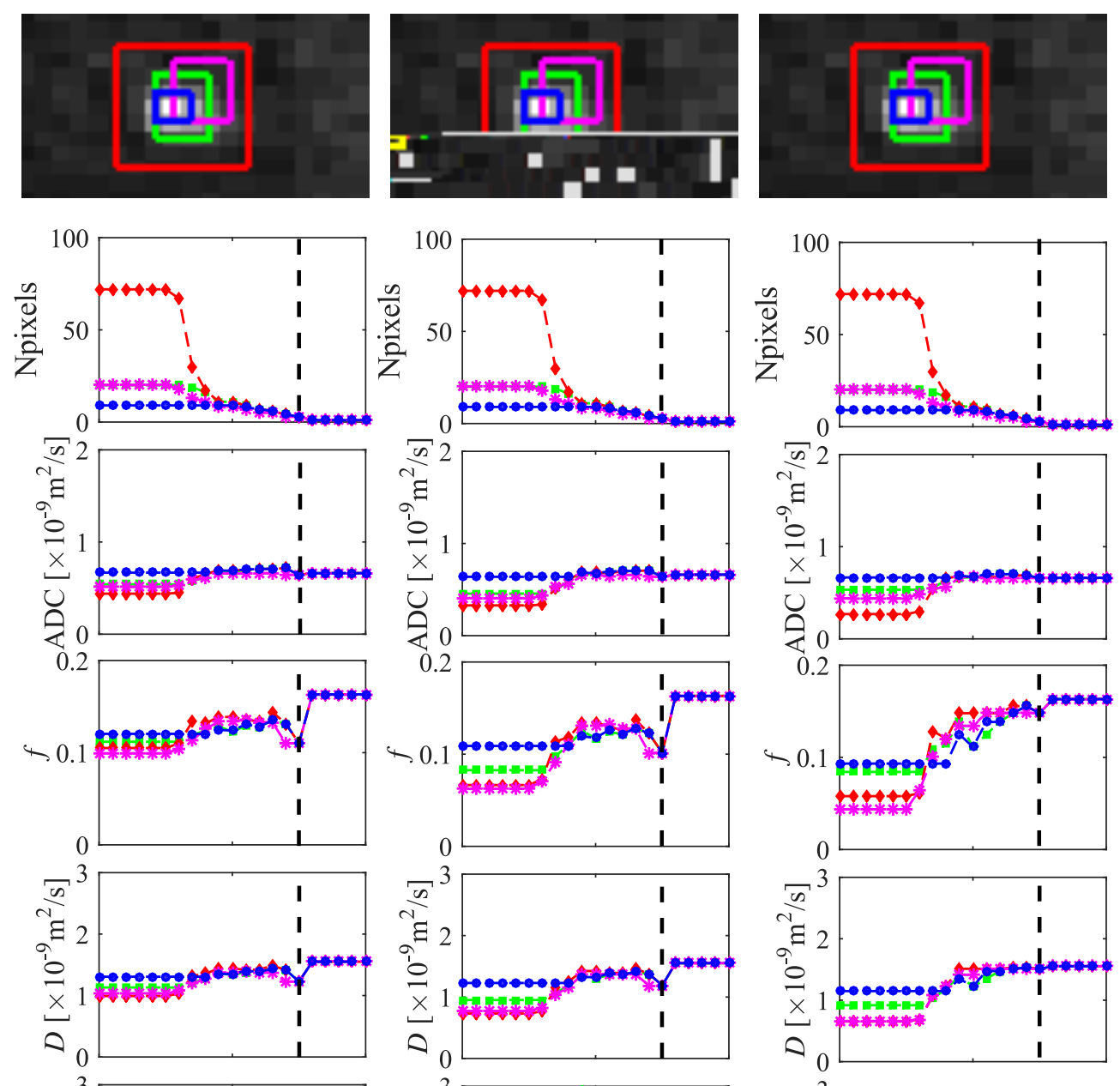

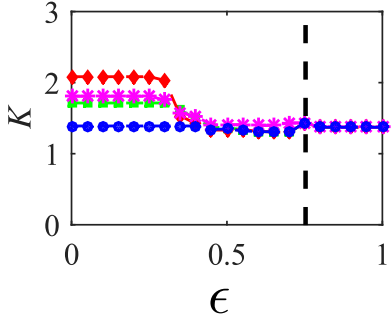

(a)

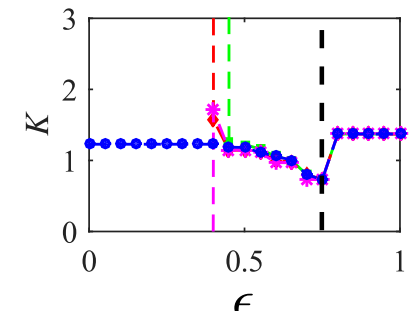

(b)

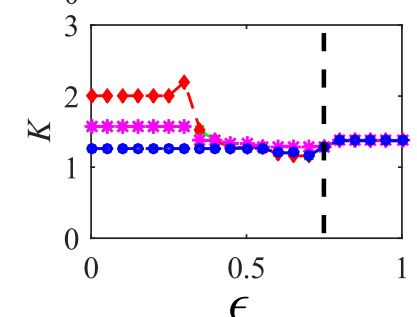

(c)

Figure 6.3: Data evolution for Npixels, $\mathrm{ADC}, f, D$ and $K$ values of the lesion in Figure $6.1(\mathrm{~b})$ as a function of thresholds based on $\mathrm{ROI}_{1}(\square), \mathrm{ROI}_{2}(\diamond), \mathrm{ROI}_{3}(*)$ and $\mathrm{ROI}_{4}(\bullet)$ on the b1500 image, using three different measurements: (a) Averaging signal; (b) Pixelwise Mean; (c) Pixelwise Median. Vertical black dash lines indicate $\epsilon=0.75$. 
$\mathrm{ROI}_{4}$ defined by two independent radiologists. Although local variations were seen in the three measurements, all differences vanished when the threshold approach to 0.85 . However, it was surprising that the difference of the mean $K$ values was much larger, may indicating insufficient data points used for fitting $K$ values.
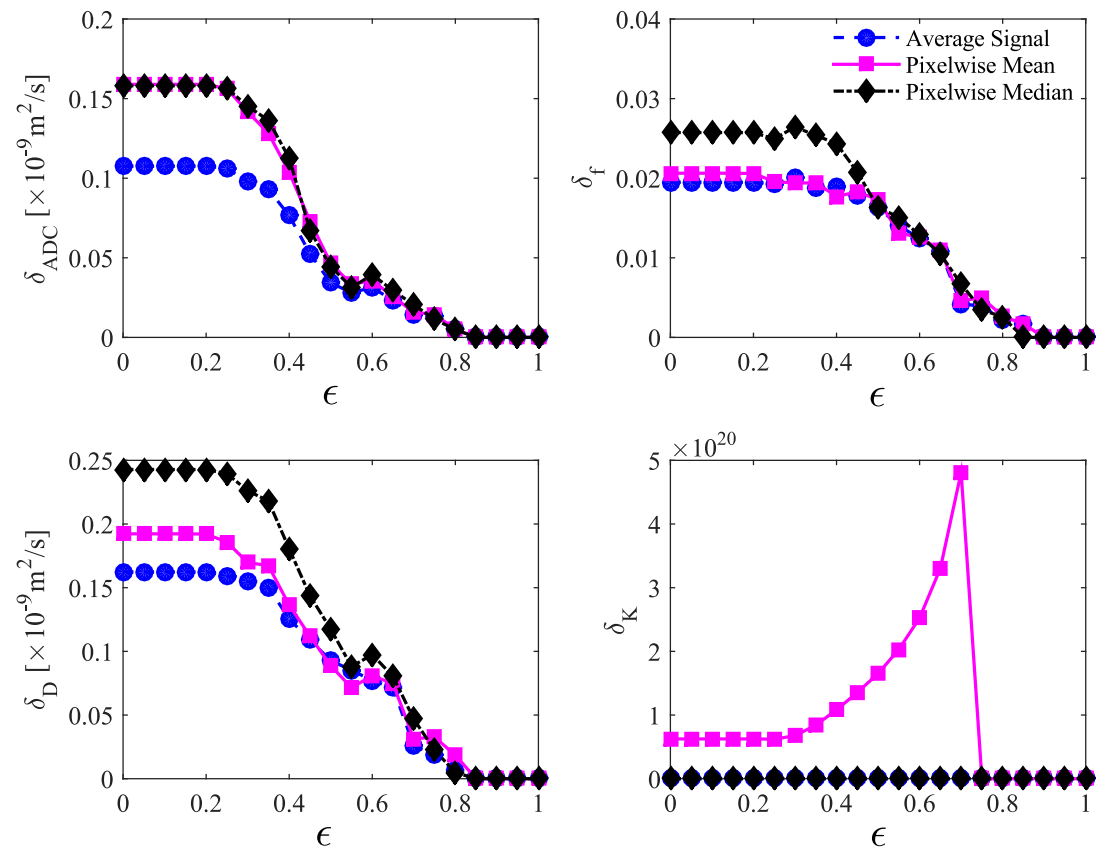

Figure 6.4: Averaged reading differences from the two independent radiologists.

\subsubsection{Statistical results}

By using the histopathological information obtained from the biopsy as a reference, the diagnostic accuracy of the diffusion parameters at different threshold values can be compared. The changes of the AUC values versus thresholds were thus calculated and shown in Figure 6.5 for the four parameters. AUC of ADC varied from 0.53 to 0.99 , and its maximum occurred when threshold was 0.85 when "Average Signal" was used. Whilst, AUC of $D$ varied from 0.55 to 0.95 , and rose to its maximum when threshold was 
0.8 or by using "Average signal" again. However, the AUC of $K$ kept constantly larger (=0.86, "Averaging Signal") when the threshold was smaller than 0.2 compared to the rest of the threshold levels. More interestingly, the best AUC of $f$ was 0.67 , much lower than other parameters, meaning that in this study, $f$ was the least accurate choice in differentiating benign and malignant lesions. Nevertheless, the "Average Signal" returned better performance, as compared to "Pixelwise Mean" or "Pixelwise Median" when the threshold was small. As the threshold approached 0.9 , the algorithm only selected one or two pixels for calculation, thus no differences in the three averaging strategies were seen.
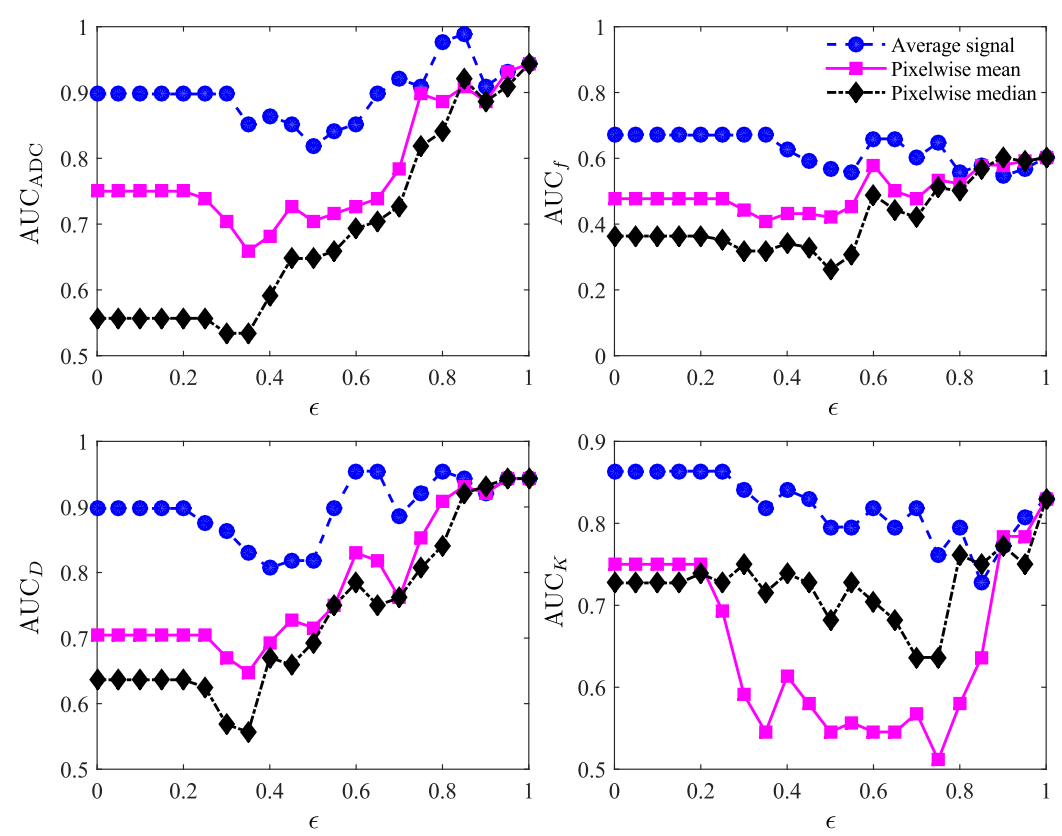

Figure 6.5: The relationship of the AUC values with different thresholds.

Boxplots of the calculated ADC, $f, D$ and $K$ values at certain thresholds are shown in Figure 6.6. It elicited that differences across thresholds were considerable, with the most stretching box presenting in the $K$ values. In particular, the boxes for both lesions were spread widely when a threshold of 1 was applied. Under the threshold of 0.85 , no outliers were shown 
except for $K$. Moreover, with this threshold, a clear separation of the ADC value in malignant lesions than in benign lesions can be seen in Figure 6.6, with "Averaging signal" shows better capability of discrimination. A separation of lesion histology appears to be possible based on $D$ and $K$. However, the $f$ values of malignant and benign lesions show little separation.
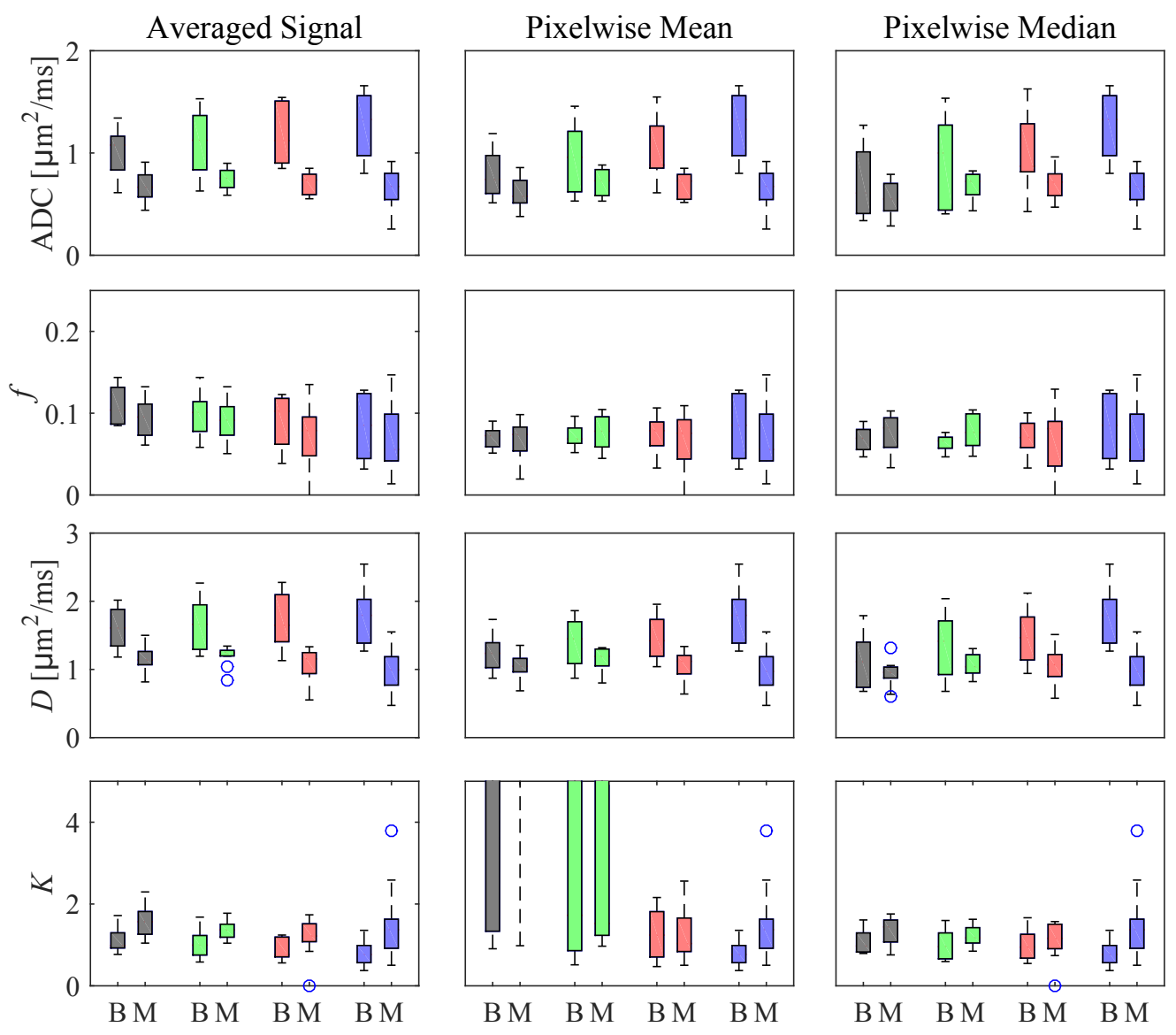

Figure 6.6: Boxplots of calculated ADC, $f, D$ and $K$ values with the thresholds of 0 (gray), 0.4 (green), 0.85 (red) and 1 (blue). " $\mathrm{B}$ " stands for "Benign" and " $\mathrm{M}$ " for "Malignant".

Based on the previous analyses, ADC was found to deliver the largest differentiation between benign and malignant lesions among the four diffusion parameters in this field study. Although the two-sample student's 
test revealed a significant difference of $D$ between benign and malignant tissues which explains the little visual differences between ADC and $D$ in the boxplot of Figure 6.6, the overall AUC values of ADC were higher than that of $D$ as shown in Figure 6.5. Therefore, a detailed investigation on ADC values with a threshold of 0.85 (red boxes shown on the top panel of Figure 6.6) was exploited and the results were summarised in Table 6.1. As expected, three averaging measurements returned similar ADC values in benign and malignant lesions. However, "Average Signal" held the smallest uncertainties. Significant differences between benign and malignant tumours were presented in the three averaging measurements ( $p=0.0025$, 0.01 and 0.02 for "Averaging signal", "Pixelwise Mean" and "Pixelwise Median", respectively). By further performing the ROC analysis, the cutoff value in "Averaging signal" was found to be $0.85 \times 10^{-9} \mathrm{~m}^{2} / \mathrm{s}$ with a sensitivity of $87.5 \%$ and specificity of $90.9 \%$. The cut-off value in "Pixelwise Mean" was $0.84 \times 10^{-9} \mathrm{~m}^{2} / \mathrm{s}$ with the same sensitivity but a smaller specificity ( $81.8 \%)$. Whilst, the cut-off value in "Pixelwise Median" was found to be $0.8 \times 10^{-9} \mathrm{~m}^{2} / \mathrm{s}$ with the same sensitivity and specificity as in "Pixelwise Mean".

Table 6.1: ADC values and the corresponding sensitivities and specificities in malignant and benign lesions with a threshold of 0.85 by using three measurement methods.

\begin{tabular}{ccccc}
\hline \hline ADC $\left(\times 10^{-9} \mathrm{~m}^{2} / \mathrm{s}\right)$ & Benign & Malignant & Cut-off & Sensitivity /Specificity \\
\hline Average Signal & $1.19 \pm 0.35$ & $0.70 \pm 0.15$ & 0.85 & $87.5 \% / 90.9 \%$ \\
Pixelwise Mean & $1.08 \pm 0.47$ & $0.68 \pm 0.17$ & 0.84 & $87.5 \% / 81.8 \%$ \\
Pixelwise Median & $1.03 \pm 0.6$ & $0.72 \pm 0.25$ & 0.8 & $87.5 \% / 81.8 \%$ \\
\hline
\end{tabular}




\subsection{Discussion}

This present study compared various small-size ROIs by imposing thresholds on signal intensities of the large-size ROI based on the b1500 image, quantitatively selecting the most effective "small-size" ROI, with the optimal threshold of 0.85 on ADC, $f, D$ and $K$ values. A subsequent statistical analysis at different thresholds established an improved diagnostic accuracy of ADC values in differentiating benign and malignant breast lesions when a threshold of 0.85 and the measurement of "Average Signal" were applied.

The use of DWI data has proven to hold a high diagnostic accuracy in the identification of breast cancer [222]. Malignant lesions show lower ADC values as compared to their benign counterparts. However, the dependence of the calculated ADC values on the size of the ROI has been recognised as the biggest source of error in ADC readings [231]. The ADC values from a smaller-range ROI were found to offer better cut-off values for the differentiation of tumour histopathology [223], which is consistent with our findings. More interestingly, similar threshold value was used $(=0.78)$ in PET studies to obtain the correct tumour boundaries [226].

The effects of ROI measurements on $f, D$ and $K$ values were not investigated previously. Our study shows that these values also depended on the size of the selected ROIs and different averaging strategies. $K$ values have shown large variations with "Pixelwise Mean", and overall worse diagnostic performance as compared to ADC values, which contradict to Sun's study [232]. This may be ascribed to a smaller number and a narrower range of $b$-values used for fitting in this study.

Several limitations must be considered in interpreting the results of the present study. Firstly, the non-linear least squares algorithm (in specific, trust-region) was used to fit all parameters. Recent study on upper abdominal organs [233] showed that bayesian probability reached the highest precision and accuracy when computing $f$ as compared to other discussed 
algorithms. Moreover, $K$ is known to be sensitive to noise, thus using the advanced fitting or more data points may yield higher accuracy [234]. Datasets involved in this study were solely acquired from Siemens scanner, thus variabilities between multiple brands of scanners may also need to be investigated while using this algorithm. It should be pointed out that the population of this pilot study is limited, but reasonable for demonstrating the feasibility of the present method.

\subsection{Conclusions}

In summary, the threshold isocontouring strategy on the selected ROI is a reliable and intuitive approach that can largely reduce the influences from ROI sizes, thus applying it prior to the quantitative evaluation and statistical analysis of DWI data is suggested. The present results support that $A D C$ value was the most promising quantification parameter in providing the highest AUC values while $f$ value was least suggested in DW-mammography. As revealed by the statistic analysis, although all three averaging measurements can significantly differentiate benign tumours from cancers, "Averaging signal" was found to be the optimal strategy in this pilot study, returning the cut-off value with the highest specificity. 


\section{Chapter 7}

\section{Conclusions}

This thesis reports on the developments of cost-effective methodologies for characterising biological tissues. To this end, various novel techniques from both MR imaging and spectroscopy have been explored. These methods not only facilitate the identification of distinctive structures and features in tissues, but also highlight the significance of applying contrast agent free NMR protocol in practise.

\subsection{Summary of original research in this thesis}

Firstly, three image reconstruction algorithms all based on a dedicated database were presented. These new methods include 1D-PCA-CS, 1DPCA-RR and 2D-PCA-RR which allows tissue features to be identified with fewer sampling points. In addition, the wavelet-CS algorithm was served as a comparison with respect to PCA based methods. In implementing these three algorithms, a $k$-space dataset was randomly undersampled, which in the case of zero-filling FT reconstruction resulted in a blurred image. However, if this image was projected into a database-driven principal component basis (1D-PCA-CS), the blurred features became much clearer by selecting components with large weighing factors. In order to overcome the issues arising from the CS sampling limit, 1D-PCA-RR was proposed 
by incorporating a pattern recognition algorithm with the reconstruction procedure. Furthermore, by extending 1D-PCA-RR to 2D-PCA-RR, it is possible to reduce the reconstruction time as $2 \mathrm{D}$ datasets are not required to be converted to $1 \mathrm{D}$ vectors. As these three PCA based algorithms rely on the database, the reconstruction results depend on whether the corresponding fully sampled image is included in the database. As discussed in Chapter 4, in case I where the fully sampled image was available in the database, 1DPCA-RR and 2D-PCA-RR returned the exact image from the database, thus the undersampled dataset was perfectly recovered. Whereas in case II, the fully sampled image was not available in the database, both 1D-PCA-RR and 2D-PCA-RR selected a set of images most similar to the undersampled image and used this set to fill in the un-sampled $k$-space. Although in this case, no methods were able to reconstruct the exact image as fully sampled, 1D-PCA-RR and 2D-PCA-RR were shown to perform better than 1D-PCA-CS and wavelet-CS. Finally, in case III, these algorithms were used to reconstruct a dataset which was generated by locally modifying the fully sampled image from case I. However, this new image was not included in the database. For these comparisons, all PCA based methods were shown to perform better than the wavelet-CS algorithm even at low sampling rates. In particular, case III closely simulated a common situation in clinical studies, where follow-up scans of patients might appear different from the previous image. Speed and feasibility of PCA based methods in MRI were demonstrated in both carrot and brain databases. In future applications, these proposed algorithms can be utilised to quickly locate specific features, such as suspicious lesions in clinical study.

In addition to improving imaging algorithms, this thesis has improved the ability of the DDCOSY method to provide excellent tissue differences which does not require the injection of contrast agents. Both numerical simulations and experiments were implemented in Chapter 5. In specific, random walk simulations and the NMR responses in four different systems were studied, yielding unique features in the four 2D $D-D$ maps. The signal 
decay of DDCOSY was re-evaluated by treating the diffusion coefficients as tensor expansion instead of a scalar value, which was learnt from DTI. Isotropic and anisotropic features were characterised by diagonal and offdiagonal peaks in the extended DDCOSY distributions. Furthermore, the eigenvalues of the diffusion tensor were extracted from the orthogonal apparent coefficients by applying three DDCOSY experiments with the gradient scheme proposed in Chapter 5 and summarised below. The first PFG pair of each DDCOSY experiment was applied on a coordinate axis and the second pair was on the diagonal axis in the perpendicular plane. Diffusion tensor matrices and FA values were calculated as a sample average. Through the analysis of the experimental results, it was shown that the elongated shapes of the cells in a carrot led to more freedom of water molecules in the growing direction. The similar degree of restrictions in the perpendicular plane demonstrated a radially symmetric structure in the carrot. Moreover, the structural differences in healthy and tumour-bearing brain tissues were observed by comparing the mean FA values and the peak patterns in the three $D$ - $D$ maps. The healthy mouse brains were demonstrated to have more anisotropic structures while the tumour-bearing mouse brain contained certain isotropic structure. These experimental results supported that this methodology enables both macroscopic and microscopic investigations of the spatial structures of a tissue, with the aid of interpreting three $D-D$ maps and deriving sample-average FA values. Furthermore, more isotropic patterns in the $D$ - $D$ map of the tumour-bearing brain were found as compared to the healthy brain in our study, indicating that the isotropic pattern in the $D$ - $D$ map may assist in diagnosing the tumour globally. Therefore, the offered approach can be potentially used in medical investigations when imaging protocols are inaccessible.

While diffusion NMR can distinguish between tissues, it is certainly not restricted to bulk measurements. Diffusion imaging techniques offer excellent human tissue contrasts routinely used in cancer research. Therefore, a 
pilot study of human breast data which was led by the German Cancer Research Centre (Deutsches Krebsforschungszentrum, Heidelberg, Germany) was subsequently reported in this thesis. This study analysed datasets from 23 female participants with suspicious lesions, acquired from the DWI protocol with various $b$-values. By utilising three diffusion models that were previously proposed, the variabilities of $\mathrm{ADC}, f, D$ and $K$ in breast lesions with different ROI selections were evaluated, and consequently a threshold isocontouring strategy was introduced to reduce the influence of the ROI sizes. Values of ADC, $f, D$ and $K$ were found to be dependent on the ROI selected for measurement initially, but showed no differences after choosing an approximate threshold of 0.85 . Similar threshold value is commonly used in PET analysis, highlighting the most significant finding in this pilot study. Besides, it was found that a small ROI and averaging signal returned a high diagnostic accuracy level. Thus, the results from this field study strengthened the idea of applying a threshold isocontouring strategy on the selected ROI prior to the quantitative assessment of diffusion weighted mammography data. This idea may assist with the standardisation of parameters in the clinical work flow.

\subsection{Recommendations for future work}

The results presented in this thesis are limited, which may be the subject of future research. For instance, the performances of the PCA based reconstruction algorithms depend on the quality of the database. In future work, a database can be expanded over time (i.e. self-learning), may resulting in better performances of these algorithms.

In the characterisation of tumour in tissue, a decreasing tumour-to-total volume ratio may return the same FA values and isotropic patterns may not exist in $D-D$ maps. However, different means of volume selection, for instance as offered by NMR methodologies at low magnetic field strength or appropriate 3D slice selection schemes [207], may address these partial 
volume effects. Hence, it is possible to envision scanning devices for medicine and material science returning sample-average fractional anisotropy at affordable prices.

As the pilot study included limited population of participants, the fluctuations were seen in the AUC curves. In addition, the fitting algorithm in that study was set to be the same for IVIM and kurtosis models, which may not have been the perfect choice. Recent publication [233, 234] suggested optimised procedures in fitting the perfusion factor and kurtosis. With a larger population and the use of advanced regression algorithms, the AUC curves may be fluctuated less and the threshold-isocontourred ROI may achieve higher diagnostic accuracy.

Apart from further investigations concerning the limitations presented in this thesis, a few fresh thoughts may excite future developments with low-cost NMR/MRI devices:

- As the random undersampling in Cartesian coordinates is simple and easy to implement, the presented image reconstruction algorithm packages can be extended to MRI techniques at low magnetic field strength, with an available high-resolution database of fully sampled images built from instruments with high magnetic field strength. Apparatuses with low magnetic field strengths are usually built from permanent magnets, thus operating prices can be dramatically cut down.

- A short version of DDCOSY (sDDCOSY) scheme was published in 2011 [143]. It would be an interesting study to migrate the proposed gradient scheme from this thesis to sDDCOSY pulse sequences and subsequently obtain FA and $\mu \mathrm{FA}$ values of materials. The challenge is to cancel the mixing term in the signal attenuation equation as presented in [143]. Similar to the DTI pulse sequence using three directions of gradients at once, a 3D experiment of sDDCOSY and its corresponding data processing toolbox may be developed to improve 
the investigation of water diffusion behaviours.

- As the reader may be aware, the diffusion kurtosis information inherently exists in the signal decay of DDCOSY, therefore a new form of the signal evolution equation considering the kurtosis term may be needed and a new data processing method may provide the information of non-Gaussian behaviours of water movements in tissues.

- Over the last few years, spectroscopic imaging has aroused appreciable interest for the diagnosis of tissue lesions by localising the region of interest and acquiring the spectrum within the local region [182]. In this context, a combination of the DDCOSY schemes and 2D-PCA-RR may be possible to characterise tissue types and elicit underlying structures more rapidly.

\subsection{Final remarks}

In general, this thesis has revolved around MR acquisition and processing advances in characterising various types of tissues. Along this line of thinking, three scientific projects were carried out independently, including the implementation of fast image reconstruction algorithms (1DPCA-CS, 1D-PCA-RR and 2D-PCA-RR), the extraction of mean FA values from bulk measurements and the selection of regions and averaging methods for improving diagnostic accuracy. These progresses are believed to be valuable contributions to the multi-disciplinary research between physics, engineering, biology, medicine, and more broadly, material sciences. The improvements as presented in thesis can stimulate future research themes and worldwide collaborations as well. 


\section{Bibliography}

[1] I. I. Rabi, J. R. Zacharias, S. Millman, and P. Kusch, "A new method of measuring nuclear magnetic moment," Phys. Rev., vol. 53, p. 318, 1938.

[2] F. Bloch, "Nuclear induction," Phys. Rev., vol. 70, pp. 460-474, 1946.

[3] E. M. Purcell, H. C. Torrey, and R. V. Pound, "Resonance absorption by nuclear magnetic moments in a solid," Phys. Rev., vol. 69, pp. 3738, 1946 .

[4] E. Hahn, "Nuclear induction due to free larmor precession," Phys. Rev., vol. 77, no. 2, p. 297, 1950.

[5] R. Damadian, "Tumor detection by nuclear magnetic resonance," Science, vol. 171, no. 3976, pp. 1151-1153, 1971.

[6] P. Mansfield and I. L. Pykett, "Biological and medical imaging by NMR," J. Magn. Reson., vol. 29, p. 355, 1978.

[7] P. A. Bottomley, "Spatial localization in nmr spectroscopy in vivo," Ann. New York Acad. Sci., vol. 508, no. 1, pp. 333-348, 1987.

[8] S. Flibotte, R. Menon, A. MacKay, J. Hailey, et al., "Proton magnetic resonance of western red cedar," Wood Fiber Sci., vol. 22, no. 4, pp. 362376, 1990. 
[9] R. R. Ernst and W. A. Anderson, "Application of fourier transform spectroscopy to magnetic resonance," Rev. Sci. Instrum., vol. 37, pp. 93-102, 1966.

[10] W. P. Aue, E. Bartholdi, and R. R. Ernst, "Two-dimensional spectroscopy. application to nuclear magnetic resonance," J. Chem. Phys., vol. 64, pp. 2229-2246, 1976.

[11] P. C. Lauterbur, "Image formation by induced local interactions: examples employing nuclear magnetic resonance," Nature, vol. 242, pp. 190-191, 1973.

[12] P. Mansfield and P. K. Grannell, "NMR 'diffraction' in solids," J. Phys. C Solid St. Phys., vol. 6, no. 22, pp. L422-L426, 1973.

[13] C. Westbrook and C. K. Roth, MRI in Practice. John Wiley \& Sons, 2011.

[14] M. Hutchinson and U. Raff, "Fast MRI data acquisition using multiple detectors," Magn. Reson. Med., vol. 6, no. 1, pp. 87-91, 1988.

[15] J. W. Carlson and T. Minemura, "Imaging time reduction through multiple receiver coil data acquisition and image reconstruction," Magn. Reson. Med., vol. 29, no. 5, pp. 681-687, 1993.

[16] K. P. Pruessmann, M. Weiger, M. B. Scheidegger, and P. Boesiger, "SENSE: sensitivity encoding for fast MRI," Magn. Reson. Med., vol. 42, no. 5, pp. 952-962, 1999.

[17] M. A. Griswold, P. M. Jakob, R. M. Heidemann, M. Nittka, V. Jellus, J. Wang, B. Kiefer, and A. Haase, "Generalized autocalibrating partially parallel acquisitions (GRAPPA)," Magn. Reson. Med., vol. 47, no. 6, pp. 1202-1210, 2002.

[18] D. Larkman and R. Nunes, "Parallel magnetic resonance imaging," Phys. Med. Biol., vol. 52, no. 7, p. R15, 2007. 
[19] E. J. Candès and J. K. Romberg, "Signal recovery from random projections," in Proc. SPIE 5674, Computational Imaging III, pp. 76-86, San Jose, United States, 2005.

[20] E. J. Candès, "Compressive sampling," in Intern. Congr. Math., vol. 3, pp. 1433-1452, Madrid, Spain, 2006.

[21] E. J. Candès, J. Romberg, and T. Tao, “Robust uncertainty principles: Exact signal reconstruction from highly incomplete frequency information," IEEE Trans. Inf. Theory, vol. 52, no. 2, pp. 489-509, 2006.

[22] D. Donoho, "Compressed sensing," IEEE Trans. Inf. Theory, vol. 52, no. 4, pp. 1289-1306, 2006.

[23] M. Lustig, D. Donoho, and J. M. Pauly, "Sparse MRI: The application of compressed sensing for rapid MR imaging," Magn. Reson. Med., vol. 58, no. 6, pp. 1182-1195, 2007.

[24] M. Lustig, D. Donoho, J. Santos, and J. Pauly, "Compressed sensing MRI," IEEE Signal Process. Mag., vol. 25, no. 2, pp. 72-82, 2008.

[25] H. Wang, Y. Miao, K. Zhou, Y. Yu, S. Bao, Q. He, et al., "Feasibility of high temporal resolution breast DCE-MRI using compressed sensing theory," Med. Phys., vol. 37, no. 9, pp. 4971-4981, 2010.

[26] T. Wech, A. Lemke, D. Medway, L.-A. Stork, C. A. Lygate, S. Neubauer, et al., "Accelerating cine-MR imaging in mouse hearts using compressed sensing," J. Magn. Reson. Imaging, vol. 34, no. 5, pp. 1072-1079, 2011.

[27] P. Caravan, J. J. Ellison, T. J. McMurry, and R. B. Lauffer, “Gadolinium (III) chelates as MRI contrast agents: Structure, dynamics, and applications.," Chem. Rev., vol. 99, pp. 2293-352, 1999. 
[28] S. D. Wolff and R. S. Balaban, "Magnetization transfer contrast (MTC) and tissue water proton relaxation in vivo," Magn. Reson. Med., vol. 10, no. 1, pp. 135-144, 1989.

[29] K. Ward, A. Aletras, and R. Balaban, "A new class of contrast agents for mri based on proton chemical exchange dependent saturation transfer (CEST)," J. Magn. Reson., vol. 143, no. 1, pp. 79-87, 2000.

[30] L. L. Latour, K. Svoboda, P. P. Mitra, and C. H. Sotak, "Timedependent diffusion of water in a biological model system," Proc. Natl. Acad. Sci. U S A, vol. 91, no. 4, pp. 1229-1233, 1994.

[31] P. J. Basser, "Inferring microstructural features and the physiological state of tissues from diffusion-weighted images.," NMR Biomed., vol. 8, no. 7-8, pp. 333-344, 1995.

[32] Y. Guo, Y.-Q. Cai, Z.-L. Cai, Y.-G. Gao, N.-Y. An, L. Ma, S. Mahankali, and J.-H. Gao, "Differentiation of clinically benign and malignant breast lesions using diffusion-weighted imaging," J. Magn. Reson. Imaging, vol. 16, no. 2, pp. 172-178, 2002.

[33] E. O. Stejskal, "Use of spin echoes in a pulsed magnetic-field gradient to study anisotropic, restricted diffusion and flow," J. Chem. Phys., vol. 43, no. 10, pp. 3597-3603, 1965.

[34] P. Basser, J. Mattiello, and D. Le Bihan, "MR diffusion tensor spectroscopy and imaging," Biophys. J., vol. 66, no. 1, pp. 259-267, 1994.

[35] S. Ma, W. Yin, Y. Zhang, and A. Chakraborty, "An efficient algorithm for compressed MR imaging using total variation and wavelets," in IEEE Comput. Soc. Conf. Comput. Vis. Pattern. Recogn., pp. 1-8, Anchorage, United States, 2008.

[36] B. Bilgic, I. Chatnuntawech, K. Setsompop, S. F. Cauley, L. L. Wald, and E. Adalsteinsson, "Fast DSI reconstruction with trained dictionaries," in 20th Int. Soc. Magn. Reson. Med., vol. 21, p. 93765, 2013. 
[37] D. K. Jones and M. Cercignani, "Twenty-five pitfalls in the analysis of diffusion MRI data," NMR Biomed., vol. 23, no. 7, pp. 803-820, 2010.

[38] F. Szczepankiewicz, S. Lasič, D. van Westen, P. C. Sundgren, E. Englund, C.-F. Westin, et al., "Quantification of microscopic diffusion anisotropy disentangles effects of orientation dispersion from microstructure: applications in healthy volunteers and in brain tumors," Neuroimage, vol. 104, pp. 241-252, 2015.

[39] N. AlRashidi, T. Gagliardi, T. Ahearn, T. Redpath, and F. Gilbert, "Effect of region of interest size in quantitative diffusion-weighted magnetic resonance imaging of the breast," Breast Cancer Res., vol. 12, no. Suppl. 3, p. P19, 2010.

[40] A. Abragam, The principles of nuclear magnetism. Oxford University Press, 1961.

[41] C. P. Slichter, Principles of magnetic resonance, with examples from solid state physics. Harper \& Row, 1963.

[42] H. Torrey, "Bloch equations with diffusion terms," Phys. Rev., vol. 104, no. 3, pp. 563-565, 1956.

[43] N. Bloembergen, E. M. Purcell, and R. V. Pound, "Relaxation effects in nuclear magnetic resonance absorption," Phys. Rev., vol. 73, pp. 679$712,1948$.

[44] P. A. Bottomley, T. H. Foster, R. E. Argersinger, and L. M. Pfeifer, "A review of normal tissue hydrogen NMR relaxation times and relaxation mechanisms from 1-100 MHz: dependence on tissue type, NMR frequency, temperature, species, excision, and age," Med. Phys., vol. 11, no. 4, pp. 425-448, 1984.

[45] P. Bottomley, C. Hardy, R. Argersinger, and G. Allen-Moore, "A review of ${ }^{1} \mathrm{H}$ nuclear magnetic resonance relaxation in pathology: are $T_{1}$ and $T_{2}$ diagnostic," Med. Phys., vol. 14, no. 1, pp. 1-37, 1987. 
[46] G. J. Stanisz, E. E. Odrobina, J. Pun, M. Escaravage, S. J. Graham, M. J. Bronskill, and R. M. Henkelman, " $t_{1}, t_{2}$ relaxation and magnetization transfer in tissue at 3T," Magn. Reson. Med., vol. 54, no. 3, pp. 507-512, 2005.

[47] E. Lammentausta, P. Kiviranta, M. Nissi, M. Laasanen, I. Kiviranta, M. Nieminen, and J. Jurvelin, " $t_{2}$ relaxation time and delayed gadolinium-enhanced MRI of cartilage (dGEMRIC) of human patellar cartilage at 1.5T and 9.4T: Relationships with tissue mechanical properties," J. Orthop. Res., vol. 24, no. 3, pp. 366-374, 2006.

[48] R. C. van de Ven, B. Hogers, A. M. van den Maagdenberg, H. J. de Groot, M. D. Ferrari, R. R. Frants, R. E. Poelmann, L. van der Weerd, and S. R. Kiihne, " $t_{1}$ relaxation in in vivo mouse brain at ultra-high field," Magn. Reson. Med., vol. 58, no. 2, pp. 390-395, 2007.

[49] W. Jost, Diffusion in solids, liquids, gases. Academic Press, 1960.

[50] I. Lowe and R. Norberg, "Free-induction decays in solids," Phys. Rev., vol. 107, no. 1, p. 46, 1957.

[51] E. L. Hahn, “Spin echoes," Phys. Rev., vol. 80, no. 4, p. 580, 1950.

[52] G. Bodenhausen, R. Freeman, and G. A. Morris, "A simple pulse sequence for selective excitation in fourier transform NMR," J. Magn. Reson., vol. 23, no. 1, pp. 171-175, 1976.

[53] J. M. S. Hutchinson, R. J. Sutherland, and M. J. R, “NMR imaging: image recovery under magnetic fields with large non-uniformities," J. Phys. E Sci. Instrum., vol. 11, pp. 217-221, 1978.

[54] P. Mansfield and P. K. Grannell, "'diffraction" and microscopy in solids and liquids by NMR," Phys. Rev. B, vol. 12, no. 9, pp. 3618-3634, 1975. 
[55] S. Ljunggren, "A simple graphical representation of fourier-based imaging methods," J. Magn. Reson., vol. 54, no. 2, pp. 338-343, 1983.

[56] A. Kumar, D. Welti, and R. R. Ernst, "NMR Fourier zeugmatography," J. Magn. Reson., vol. 18, no. 1, pp. 69-83, 1975.

[57] R. R. Ernst, G. Bodenhausen, and A. Wokaun, Principles of nuclear magnetic resonance in one and two dimensions. Clarendon Press, Oxford, 1987.

[58] K. Rao, D. Kim, and J. Hwang, Fast fourier transform-algorithms and applications. Springer Netherlands, 2010.

[59] P. Mansfield, "Planar spin imaging by NMR," J. Magn. Reson., vol. 10, pp. L55-L58, 1977.

[60] W. Edelstein, J. Hutchison, G. Johnson, and T. Redpath, "Spin warp NMR imaging and applications to human whole-body imaging," Phys. Med. Biol., vol. 25, no. 4, p. 751, 1980.

[61] A. K. Louis, “Optimal sampling in nuclear magnetic resonance (NMR) tomography," J. Comput. Assist. Tomogr., vol. 6, no. 2, pp. 334-340, 1982.

[62] G. Glover and J. Pauly, "Projection reconstruction techniques for reduction of motion effects in MRI," Magn. Reson. Med., vol. 28, no. 2, pp. 275-289, 1992.

[63] C. H. Meyer, B. S. Hu, D. G. Nishimura, and A. Macovski, "Fast spiral coronary artery imaging," Magn. Reson. Med., vol. 28, no. 2, pp. 202-213, 1992.

[64] J. Tanner, "Use of the stimulated echo in NMR diffusion studies," J. Chem. Phys., vol. 52, no. 5, pp. 2523-2526, 1970. 
[65] P. T. Callaghan, Principles of nuclear magnetic resonance microscopy. Oxford University Press, 1991.

[66] P. T. Callaghan, Translational dynamics and magnetic resonance: principles of pulsed gradient spin echo NMR. Oxford University Press, 2011.

[67] D. Cory, "Measurement of translational displacement probabilities by NMR: an indicator of compartmentation," Magn. Reson. Med., vol. 14, no. 3, pp. 435-444, 1990.

[68] A. Einstein, Investigations on the Theory of the Brownian Movement. Courier Corporation, 1956.

[69] O. Reynolds, "An experimental investigation of the circumstances which determine whether the motion of water shall be direct or sinuous, and of the law of resistance in parallel channels," Phil Trans Roy Soc Lond, vol. 174, pp. 935-982, 1883.

[70] J. Crank, The mathematics of diffusion. Oxford university press, 1979.

[71] J. Kärger and W. Heink, "The propagator representation of molecular transport in microporous crystallites," J. Magn. Reson., vol. 51, pp. 1-7, 1983.

[72] P. T. Callaghan, C. D. Eccles, and Y. Xia, "NMR microscopy of dynamic displacements: $k$-space and $q$-space imaging," J. Phys. E, vol. 21, no. 8, pp. 820-822, 1988.

[73] F. B. Laun, T. A. Kuder, W. Semmler, and B. Stieltjes, “Determination of the defining boundary in nuclear magnetic resonance diffusion experiments," Phys. Rev. Lett., vol. 107, p. 048102, 2011.

[74] S. Hertel, M. Hunter, and P. Galvosas, "Magnetic resonance pore imaging, a tool for porous media research," Phys. Rev. E, vol. 87, p. 030802, 2013. 
[75] W. Kittler, P. Galvosas, and M. Hunter, "Parallel acquisition of $q$ space using second order magnetic fields for single-shot diffusion measurements," J. Magn. Reson., vol. 244, pp. 46-52, 2014.

[76] J. Stepišnik, "Analysis of NMR self-diffusion measurements by a density matrix calculation.," Physica B, vol. 104, pp. 350-364, 1981.

[77] A. Berezhkovskii and G. Sutmann, "Time and length scales for diffusion in liquids," Phys. Rev. E, vol. 65, no. 6, p. 060201, 2002.

[78] M. Appel, G. Fleischer, J. Kärger, F. Fujara, and S. Siegel, “NMR evidence of anomalous molecular diffusion due to structural confinement," Europhys. Lett., vol. 34, pp. 483-487, 1996.

[79] J. Kärger and F. Stallmach, "PFG NMR studies of anomalous diffusion," pp. 417-459, 2005.

[80] M. Holz, S. R. Heil, and A. Sacco, "Temperature dependent selfdiffusion coefficients of water and six selected molecular liquids for calibration in accurate ${ }^{1} \mathrm{H}$ NMR PFG-measurements," Phys. Chem. Chem. Phys., vol. 2, p. 4740, 2000.

[81] P. P. Mitra, P. N. Sen, and L. M. Schwartz, "Short-time behavior of the diffusion coefficient as a geometrical probe of porous media," Phys. Rev. B, vol. 47, pp. 8565-8574, 1993.

[82] J. E. Tanner, “Transient diffusion in a system partitioned by permeable barriers - application to NMR measurements with a pulsed field gradient," J. Chem. Phys., vol. 69, pp. 1748-1754, 1978.

[83] E. Von Meerwall and R. Ferguson, "Interpreting pulsed-gradient spinecho diffusion experiments with permeable membranes," J. Chem. Phys., vol. 74, no. 12, pp. 6956-6959, 1981. 
[84] T. A. Sibgatullin, F. J. Vergeldt, E. Gerkema, and H. Van As, “Quantitative permeability imaging of plant tissues," Eur. Biophys. J., vol. 39, no. 4, pp. 699-710, 2010.

[85] P. Mansfield, "Multi-planar image formation using NMR spin echoes," J. Phys. C Solid St. Phys., vol. 10, pp. L55-L58, 1977.

[86] A. Haase, J. Frahm, D. Matthaei, W. Hanicke, and K. D. Merboldt, "FLASH imaging. Rapid NMR imaging using low flip-angle pulses," J. Magn. Reson., vol. 67, no. 2, pp. 258-266, 1986.

[87] J. Hennig, A. Nauerth, and H. Friedburg, "RARE imaging: A fast imaging method for clinical MR," Magn. Reson. Med., vol. 3, pp. 823833, 1986.

[88] J. Ra and C. Rim, "Fast imaging using sub-encoding data sets from multiple detectors," Magn. Reson. Med., vol. 30, no. 1, pp. 142-145, 1993.

[89] H. Nyquist, "Certain topics in telegraph transmission theory," AIEE Trans., 1928.

[90] C. E. Shannon, "Communication in the presence of noise," In: Proc. Inst. Radio. Eng., vol. 37, no. 1, pp. 10-21, 1949.

[91] E. J. Candès and M. B. Wakin, "An introduction to compressive sampling," IEEE Signal Processing Magazine, vol. 25, no. 2, pp. 21-30, 2008.

[92] E. J. Candès and J. Romberg, "Sparsity and incoherence in compressive sampling," Inverse Probl., vol. 23, no. 3, pp. 969-985, 2007.

[93] W. Yin, S. Osher, D. Goldfarb, and J. Darbon, “Bregman iterative algorithms for $\ell_{1}$-minimization with applications to compressed sensing," SIAM J. Imaging Sci., vol. 1, no. 1, pp. 143-168, 2008. 
[94] I. Daubechies, M. Defrise, and C. De Mol, "An iterative thresholding algorithm for linear inverse problems with a sparsity constraint," Commun. Pure Appl. Math., vol. 57, no. 11, pp. 1413-1457, 2004.

[95] X. Qu, W. Zhang, D. Guo, and C. Cai, "Iterative thresholding compressed sensing MRI based on contourlet transform," Inverse Probl. Sci. Eng., vol. 18, no. 6, pp. 737-758, 2010.

[96] S. G. Mallat, "A theory for multiresolution signal decomposition: the wavelet representation," IEEE Trans. Pattern. Anal. Mach. Intell., vol. 11, no. 7, pp. 674-693, 1989.

[97] D. Taubman and M. Marcellin, JPEG2000 image compression fundamentals, standards and practice, vol. 642. Springer Science \& Business Media, 2012.

[98] A. N. Akansu and R. A. Haddad, Multiresolution signal decomposition: transforms, subbands, and wavelets. Academic Press, 2001.

[99] M. Elad and M. Aharon, "Image denoising via sparse and redundant representations over learned dictionaries," IEEE Trans. Image Process., vol. 15, no. 12, pp. 3736-3745, 2006.

[100] R. Masiero, G. Quer, D. Munaretto, M. Rossi, J. Widmer, and M. Zorzi, "Data acquisition through joint compressive sensing and principal component analysis," in Global Telecommunications Conference, IEEE, pp. 1-6, IEEE, 2009.

[101] J. Geis, R. Hendrick, S. Lee, K. Davis, and D. Thickman, "White matter lesions: role of spin density in MR imaging," Radiology, vol. 170, no. 3, pp. 863-868, 1989.

[102] W. Kucharczyk, A. P. Crawley, W. Kelly, and R. M. Henkelman, "Effect of multislice interference on image contrast in $T_{2}$-and $T_{1}$-weighted MR images," $A J N R$, vol. 9, no. 3, pp. 443-451, 1988. 
[103] G. Wesbey, K. Moon, L. Crooks, M. Arakawa, and R. Brasch, “Proton$T_{2}$ reduction due to spin diffusion through pulsed gradient in spinecho NMR imaging-imaging implications and applications," Magn. Reson. Med., vol. 1, no. 2, pp. 273-274, 1984.

[104] D. Le Bihan and E. Breton, "Imagerie de diffusion in-vivo par résonance magnétique nucléaire," C. R. Acad. Sc. Paris, vol. 93, no. 5, pp. 2734, 1985 .

[105] F. Pediconi, C. Catalano, A. Roselli, S. Padula, F. Altomari, E. Moriconi, et al., "Contrast-enhanced MR mammography for evaluation of the contralateral breast in patients with diagnosed unilateral breast cancer or high-risk lesions," Radiology, vol. 243, no. 3, pp. 670680, 2007.

[106] S. C. Partridge, H. Rahbar, R. Murthy, X. Chai, B. F. Kurland, W. B. DeMartini, et al., "Improved diagnostic accuracy of breast MRI through combined apparent diffusion coefficients and dynamic contrastenhanced kinetics," Magn. Reson. Med., vol. 65, no. 6, pp. 1759-1767, 2011.

[107] W. A. Kaiser, Signs in MR-mammography. Springer, 2008.

[108] S. Cheong, P. Ferguson, K. W. Feindel, I. F. Hermans, P. T. Callaghan, C. Meyer, et al., "Simple synthesis and functionalization of iron nanoparticles for magnetic resonance imaging," Angew. Chem. Int. Ed., vol. 50, no. 18, pp. 4206-4209, 2011.

[109] M. Bergamino, L. Bonzano, F. Levrero, G. Mancardi, and L. Roccatagliata, "A review of technical aspects of $T_{1}$-weighted dynamic contrast-enhanced magnetic resonance imaging (DCE-MRI) in human brain tumors," Physica Medica, vol. 30, no. 6, pp. 635-643, 2014.

[110] C. K. Kuhl, “The current status of breast imaging part I. choice of 
technique, image interpretation, diagnostic accuracy, and transfer to clinical practice," Radiology, vol. 244, no. 2, pp. 356-378, 2007.

[111] D. K. Jones, Diffusion MRI: theory, methods, and application. Oxford University Press, 2011.

[112] J. Mattiello, P. J. Basser, and D. Le Bihan, “The b matrix in diffusion tensor echo-planar imaging," Magn. Reson. Med., vol. 37, no. 2, pp. 292-300, 1997.

[113] J. Mattiello, P. J. Basser, and D. Le Bihan, "Analytical expressions for the $b$ matrix in NMR diffusion imaging and spectroscopy," J. Magn. Reson., vol. 108, pp. 131-141, 1994.

[114] D. Le Bihan, E. Breton, D. Lallemand, M.-L. Aubin, J. Vignaud, and M. Laval-Jeantet, "Separation of diffusion and perfusion in intravoxel incoherent motion MR imaging," Radiology, vol. 168, no. 2, pp. 497$505,1988$.

[115] E. E. Sigmund, G. Y. Cho, S. Kim, M. Finn, M. Moccaldi, J. H. Jensen, et al., "Intravoxel incoherent motion imaging of tumor microenvironment in locally advanced breast cancer," Magn. Reson. Med., vol. 65, no. 5, pp. 1437-1447, 2011.

[116] S. Suo, N. Lin, H. Wang, L. Zhang, R. Wang, S. Zhang, et al., "Intravoxel incoherent motion diffusion-weighted MR imaging of breast cancer at 3.0 Tesla: Comparison of different curve-fitting methods," J. Magn. Reson. Imaging, vol. 42, no. 2, pp. 362-370, 2015.

[117] C. Van Donkelaar, L. Kretzers, P. Bovendeerd, L. Lataster, K. Nicolay, J. Janssen, et al., "Diffusion tensor imaging in biomechanical studies of skeletal muscle function," J. Anat., vol. 194, no. 1, pp. 79-88, 1999.

[118] C. A. Wheeler-Kingshott, S. J. Hickman, G. J. Parker, O. Ciccarelli, M. R. Symms, D. H. Miller, et al., "Investigating cervical spinal cord 
structure using axial diffusion tensor imaging," Neuroimage, vol. 16, no. 1, pp. 93-102, 2002.

[119] R. Naismith, N. Tutlam, J. Xu, E. Klawiter, J. Shepherd, K. Trinkaus, et al., "Optical coherence tomography differs in neuromyelitis optica compared with multiple sclerosis," Neurology, vol. 72, no. 12, pp. 10771082, 2009.

[120] A. M. Uluğ and P. C. van Zijl, "Orientation-independent diffusion imaging without tensor diagonalization: anisotropy definitions based on physical attributes of the diffusion ellipsoid," J. Magn. Reson. Imaging, vol. 9, no. 6, pp. 804-813, 1999.

[121] A. H. Poonawalla and X. J. Zhou, "Analytical error propagation in diffusion anisotropy calculations," J. Magn. Reson. Imaging, vol. 19, no. 4, pp. 489-498, 2004.

[122] S. N. Jespersen, H. Lundell, C. K. Sønderby, and T. B. Dyrby, "Orientationally invariant metrics of apparent compartment eccentricity from double pulsed field gradient diffusion experiments," NMR in Biomedicine, vol. 26, no. 12, pp. 1647-1662, 2013.

[123] K. P. Balanda and H. MacGillivray, "Kurtosis: a critical review," Am. Stat., vol. 42, no. 2, pp. 111-119, 1988.

[124] J. H. Jensen, J. A. Helpern, A. Ramani, H. Lu, and K. Kaczynski, "Diffusional kurtosis imaging: the quantification of non-gaussian water diffusion by means of magnetic resonance imaging," Magn. Reson. Med., vol. 53, no. 6, pp. 1432-1440, 2005.

[125] J. Zhuo, S. Xu, J. L. Proctor, R. J. Mullins, J. Z. Simon, G. Fiskum, and R. P. Gullapalli, "Diffusion kurtosis as an in vivo imaging marker for reactive astrogliosis in traumatic brain injury," Neuroimage, vol. 59, no. 1, pp. 467-477, 2012. 
[126] J. H. Jensen and J. A. Helpern, "MRI quantification of non-gaussian water diffusion by kurtosis analysis," NMR Biomed., vol. 23, no. 7, pp. 698-710, 2010.

[127] J. H. Lee, C. Labadie, C. S. Springer, and G. S. Harbison, “Twodimensional inverse laplace transform NMR - altered relaxationtimes allow detection of exchange-correlation," J. Am. Chem. Soc., vol. 115, pp. 7761-7764, 1993.

[128] Y. Q. Song, L. Venkataramanan, M. D. Hürlimann, M. Flaum, P. Frulla, and C. Straley, " $T_{1}-T_{2}$ correlation spectra obtained using a fast twodimensional Laplace inversion," J. Magn. Reson., vol. 154, pp. 261-268, 2002.

[129] P. T. Callaghan, S. Godefroy, and B. N. Ryland, "Use of the second dimension in PGSE NMR studies of porous media," Magn. Reson. Imaging, vol. 21, no. 3-4, pp. 243-248, 2003.

[130] P. Galvosas and P. T. Callaghan, "Multi-dimensional inverse Laplace spectroscopy in the NMR of porous media," C. R. Physique, vol. 11, no. 2, pp. 172-180, 2010.

[131] H. Liu, M. Nogueira d'Eurydice, S. Obruchkov, and P. Galvosas, "Determining pore length scales and pore surface relaxivity of rock cores by internal magnetic fields modulation at 2MHz NMR," J. Magn. Reson., vol. 246, pp. 110-118, 2014.

[132] P. T. Callaghan and M. E. Komlosh, "Locally anisotropic motion in a macroscopically isotropic system: displacement correlations measured using double pulsed gradient spin-echo NMR," Magn. Reson. Chem., vol. 40, no. 13, pp. S15-S19, 2002.

[133] P. T. Callaghan and I. Furó, "Diffusion-diffusion correlation and exchange as a signature for local order and dynamics," J. Chem. Phys., vol. 120, no. 8, pp. 4032-4038, 2004. 
[134] P. T. Callaghan, S. Godefroy, and B. N. Ryland, "Diffusion-relaxation correlation in simple pore structures," J. Magn. Reson., vol. 162, no. 2, pp. 320-327, 2003.

[135] P. L. Hubbard, K. M. McGrath, and P. T. Callaghan, "A study of anisotropic water self-diffusion and defects in the lamellar mesophase," Langumir, vol. 21, no. 10, pp. 4340-4346, 2005.

[136] A. A. Khrapitchev and P. T. Callaghan, "Double PGSE NMR with stimulated echoes: Phase cycles for the selection of desired encoding," J. Magn. Reson., vol. 152, pp. 259-268, 2001.

[137] M. Komlosh, F. Horkay, R. Freidlin, U. Nevo, Y. Assaf, and P. Basser, "Detection of microscopic anisotropy in gray matter and in a novel tissue phantom using double pulsed gradient spin echo MR," J. Magn. Reson., vol. 189, no. 1, pp. 38-45, 2007.

[138] E. Özarslan and P. J. Basser, "Microscopic anisotropy revealed by NMR double pulsed field gradient experiments with arbitrary timing parameters," J. Chem. Phys., vol. 128, no. 15, p. 154511, 2008.

[139] E. Özarslan, "Compartment shape anisotropy (CSA) revealed by double pulsed field gradient MR," J. Magn. Reson., vol. 199, no. 1, pp. 56-67, 2009.

[140] N. Shemesh, E. Özarslan, M. E. Komlosh, P. J. Basser, and Y. Cohen, "From single-pulsed field gradient to double-pulsed field gradient MR: gleaning new microstructural information and developing new forms of contrast in MRI," NMR Biomed., vol. 23, no. 7, pp. 757-780, 2010.

[141] M. A. Koch and J. Finsterbusch, "Towards compartment size estimation in vivo based on double wave vector diffusion weighting," NMR Biomed., vol. 24, no. 10, pp. 1422-1432, 2011. 
[142] J. L. Paulsen and Y.-Q. Song, "Two-dimensional diffusion time correlation experiment using a single direction gradient," J. Magn. Reson., vol. 244, pp. 6-11, 2014.

[143] N. Spindler, Diffusion and flow investigations in natural porous media by nuclear magnetic resonance. Schriften des Forschungszentrums Jülich, 2011.

[144] L. Venkataramanan, Y.-Q. Song, and M. D. Hürlimann, "Solving Fredholm integrals of the first kind with tensor product structure in 2 and 2.5 dimensions," IEEE Trans. Signal Process., vol. 50, no. 5, pp. 1017-1026, 2002.

[145] Y. Q. Song, “Categories of coherence pathways for the CPMG sequence," J. Magn. Reson., vol. 157, pp. 82-91, 2002.

[146] A. N. Tikhonov and V. Y. Arsenin, Solutions of ill-posed problems. V.H. Winson Sons, 1977.

[147] J. P. Butler, J. A. Reeds, and S. V. Dawson, "Estimating solutions of first kind integral equations with nonnegative constraints and optimal smoothing," vol. 18, no. 3, pp. 381-397, 1981.

[148] Y. Qiao, P. Galvosas, and P. T. Callaghan, "Diffusion correlation NMR spectroscopic study of anisotropic diffusion of water in plant tissues," Biophys. J., vol. 89, no. 4, pp. 2899-2905, 2005.

[149] M. Hfirlimann, L. Venkataramanan, C. Flaum, P. Speier, C. Karmonik, R. Freedman, N. Heaton, et al., "Diffusion-editing: New NMR measurement of saturation and pore geometry," in SPWLA 43rd Annual Logging Symposium, Oiso, Japan, 2002.

[150] Y.-Q. Song, L. Venkataramanan, and L. Burcaw, "Determining the resolution of Laplace inversion spectrum.," J. Chem. Phys., vol. 122, p. 104104, 2005. 
[151] R. L. Parker and Y.-Q. Song, "Assigning uncertainties in the inversion of NMR relaxation data," J. Magn. Reson., vol. 174, no. 2, pp. 314-324, 2005.

[152] Y.-Q. Song, "Resolution and uncertainty of Laplace inversion spectrum," Magn. Reson. Imaging, vol. 25, no. 4, pp. 445-448, 2007.

[153] M. Prange and Y.-Q. Song, "Quantifying uncertainty in NMR $T_{2}$ spectra using Monte Carlo inversion," J. Magn. Reson., vol. 196, no. 1, pp. 54-60, 2009.

[154] M. Prange and Y.-Q. Song, "Understanding NMR $T_{2}$ spectral uncertainty," J. Magn. Reson., vol. 204, pp. 118-123, 2010.

[155] D. Doddrell, D. Pegg, and M. R. Bendall, "Distortionless enhancement of NMR signals by polarization transfer," J. Magn. Reson., vol. 48, no. 2, pp. 323-327, 1982.

[156] Y.-Q. Song, L. Zielinski, and S. Ryu, "Two-dimensional nmr of diffusion systems," Phys. Rev. Lett., vol. 100, p. 248002, 2008.

[157] K. Person, "On lines and planes of closest fit to system of points in space," vol. 6, 1901.

[158] J. Yang, D. Zhang, A. F. Frangi, and J.-Y. Yang, “Two-dimensional PCA: a new approach to appearance-based face representation and recognition," IEEE Trans. Pattern Anal. Mach. Intell., vol. 26, no. 1, pp. 131-137, 2004.

[159] M. Turk and A. Pentland, "Eigenfaces for recognition," J. Cognit. Neurosci., vol. 3, no. 1, pp. 71-86, 1991.

[160] J. Taur and C. Tao, "Medical image compression using principal component analysis," IEEE Trans. Image Process., vol. 1, pp. 903-906, 1996. 
[161] H. Hotelling, "Analysis of a complex of statistical variables into principal components," J. Educ. Psychol., vol. 24, no. 6, pp. 417-441, 1933.

[162] I. Jolliffe, Principal component analysis. Wiley Online Library, 2002.

[163] J. T. Brindle, H. Antti, E. Holmes, G. Tranter, J. K. Nicholson, H. W. Bethell, et al., "Rapid and noninvasive diagnosis of the presence and severity of coronary heart disease using ${ }^{1} \mathrm{H}-\mathrm{NMR}$-based metabonomics," Nature Med., vol. 8, no. 12, pp. 1439-1445, 2002.

[164] J. Velikina, A. Alexander, and A. Samsonov, "A novel approach for $T_{1}$ relaxometry using constrained reconstruction in parametric dimension," in 18th Int. Soc. Magn. Reson. Med., vol. 18, p. 350, 2010.

[165] C. Huang, C. G. Graff, E. W. Clarkson, A. Bilgin, and M. I. Altbach, " $T_{2}$ mapping from highly undersampled data by reconstruction of principal component coefficient maps using compressed sensing," Magn. Reson. Med., vol. 67, no. 5, pp. 1355-1366, 2012.

[166] U. Gamper, P. Boesiger, and S. Kozerke, "Compressed sensing in dynamic MRI," Magn. Reson. Med., vol. 59, no. 2, pp. 365-373, 2008.

[167] H. Pedersen, S. Kozerke, S. Ringgaard, K. Nehrke, and W. Y. Kim, " $k$ - $t$ PCA: temporally constrained $k$ - $t$ BLAST reconstruction using principal component analysis," Magn. Reson. Med., vol. 62, no. 3, pp. 706-716, 2009.

[168] J. Tsao, P. Boesiger, and K. P. Pruessmann, " $k-t$ BLAST and $k-t$ SENSE: Dynamic MRI with high frame rate exploiting spatiotemporal correlations," Magn. Reson. Med., vol. 50, no. 5, pp. 1031-1042, 2003.

[169] J. V. Velikina and A. a. Samsonov, "Reconstruction of dynamic image series from undersampled MRI data using data-driven model 
consistency condition (MOCCO)," Magn. Reson. Med., vol. 74, no. 5, pp. 1279-1290, 2015.

[170] Y. Zhou, Y. Wang, and L. Ying, "A kernel-based compressed sensing approach to dynamic MRI from highly undersampled data," in 10th IEEE Int. Symp. Biomed. Imaging, pp. 310-313, 2013.

[171] E. Pierre, N. Seiberlich, S. Yutzy, J. Tkach, and M. Griswold, "Atlas based sparsification of image and theoretical estimation (ABSINTHE)," in 17th Int. Soc. Magn. Reson. Med., vol. 17, p. 4554, 2009.

[172] E. Pierre, N. Seiberlich, S. Yutzy, J. Tkach, V. Gulani, F. Breuer, et al., "Iterative approach to atlas based sparsification of image and theoretical estimation (iterative ABSINTHE)," in 18th Int. Soc. Magn. Reson. Med., vol. 18, p. 2875, 2010.

[173] O. Dietrich, A. Hubert, and S. Heiland, "Imaging cell size and permeability in biological tissue using the diffusion-time dependence of the apparent diffusion coefficient," Phys. Med. Biol., vol. 59, no. 12, pp. 3081-3096, 2014.

[174] D. Güllmar, T. Jaap, M. E. Bellemann, J. Haueisen, and J. R. Reichenbach, "DTI measurements of isotropic and anisotropic media," Biomed. Tech. Berl., vol. 47, no. Suppl 1, pp. 420-422, 2002.

[175] S. Mori, R. Itoh, J. Zhang, W. E. Kaufmann, P. C. van Zijl, M. Solaiyappan, et al., "Diffusion tensor imaging of the developing mouse brain," Magn. Reson. Med., vol. 46, no. 1, pp. 18-23, 2001.

[176] J. Zhang, P. C. van Zijl, and S. Mori, “Three-dimensional diffusion tensor magnetic resonance microimaging of adult mouse brain and hippocampus," Neuroimage, vol. 15, no. 4, pp. 892-901, 2002.

[177] G. Paxinos and K. B. Franklin, The mouse brain in stereotaxic coordinates. Gulf Professional Publishing, 2004. 
[178] J. Zhang, L. J. Richards, M. I. Miller, P. Yarowsky, P. van Zijl, and S. Mori, "Characterization of mouse brain and its development using diffusion tensor imaging and computational techniques," in 28th Int. Conf. IEEE Eng. Med. Biol. Soc., vol. 1, pp. 2252-2255, 2006.

[179] E. Morris and L. Liberman, Breast MRI: Diagnosis and Intervention. Springer, Cambridge, 2005.

[180] American College of Radiology, Breast imaging reporting and data system. American College of Radiology, 1998.

[181] D. Ma, V. Gulani, N. Seiberlich, K. Liu, J. L. Sunshine, J. L. Duerk, et al., "Magnetic resonance fingerprinting," Nature, vol. 495, no. 7440, pp. 187-192, 2013.

[182] P. Cao and E. X. Wu, "Accelerating phase-encoded proton MR spectroscopic imaging by compressed sensing," J. Magn. Reson. Imaging, vol. 41, no. 2, pp. 487-495, 2015.

[183] L. I. Rudin, S. Osher, and E. Fatemi, "Nonlinear total variation based noise removal algorithms," Physica D, vol. 60, no. 1, pp. 259-268, 1992.

[184] A. Eskicioglu and P. Fisher, "Image quality measures and their performance," IEEE Trans. Commun., vol. 43, no. 12, pp. 2959-2965, 1995.

[185] Z. Wang, A. C. Bovik, H. R. Sheikh, and E. P. Simoncelli, "Image quality assessment: from error visibility to structural similarity," IEEE Trans. Image Process., vol. 13, no. 4, pp. 600-612, 2004.

[186] R. Dosselmann and X. D. Yang, "A comprehensive assessment of the structural similarity index," Signal Image Video Process., vol. 5, no. 1, pp. 81-91, 2011. 
[187] J. L. Paulsen, H. Cho, G. Cho, and Y.-Q. Song, "Acceleration of multidimensional propagator measurements with compressed sensing," $J$. Magn. Reson., vol. 213, no. 1, pp. 166-170, 2011.

[188] I. Tošić, I. Jovanović, P. Frossard, M. Vetterli, and N. Durić, “Ultrasound tomography with learned dictionaries," in Int. Conf. IEEE Acoust. Speech. Signal. Process., pp. 5502-5505, Texas, U.S.A, 2010.

[189] "OASIS database." http://oasis-brains.org/. Accessed: $13 / 07 / 2015$.

[190] S. C. Partridge, A. Ziadloo, R. Murthy, S. W. White, S. Peacock, P. R. Eby, et al., "Diffusion tensor MRI: Preliminary anisotropy measures and mapping of breast tumors," J. Magn. Reson. Imaging, vol. 31, no. 2, pp. 339-347, 2010.

[191] P. Sundgren, Q. Dong, D. Gomez-Hassan, S. Mukherji, P. Maly, and R. Welsh, "Diffusion tensor imaging of the brain: review of clinical applications," Neuroradiology, vol. 46, no. 5, pp. 339-350, 2004.

[192] H. Mamata, F. A. Jolesz, and S. E. Maier, "Characterization of central nervous system structures by magnetic resonance diffusion anisotropy," Neurochem. Int., vol. 45, no. 4, pp. 553-560, 2004.

[193] C. E. Gounaris, E. L. First, and C. A. Floudas, "Estimation of diffusion anisotropy in microporous crystalline materials and optimization of crystal orientation in membranes," J. Chem. Phys., vol. 139, no. 12, p. 124703, 2013.

[194] L. Dervan, A. Poliakov, S. D. Friedman, D. Shaw, C. Pihoker, J. S. Roberts, T. Richards, K. Marro, and M. S. Vavilala, "Change in fractional anisotropy during treatment of diabetic ketoacidosis in children," Pediatr. Res., vol. 75, no. 1-1, pp. 62-66, 2013. 
[195] M. Lawrenz, S. Brassen, and J. Finsterbusch, “Microscopic diffusion anisotropy in the human brain: Reproducibility, normal values, and comparison with the fractional anisotropy," Neuroimage, vol. 109, pp. 283-297, 2015.

[196] P. Larvaron, O. Boespflug-Tanguy, J.-P. Renou, and J.-M. Bonny, “In vivo analysis of the post-natal development of normal mouse brain by DTI," NMR Biomed., vol. 20, no. 4, pp. 413-421, 2007.

[197] K. S. Mendelson, "Percolation model of nuclear magnetic relaxation in porous media," Phys. Rev. B, vol. 41, no. 1, p. 562, 1990.

[198] D. J. Bergman, K.-J. Dunn, L. M. Schwartz, and P. P. Mitra, “Selfdiffusion in a periodic porous medium: a comparison of different approaches," Phys. Rev. E, vol. 51, no. 4, p. 3393, 1995.

[199] L. Schwartz, D. Johnson, J. Mitchell, T. Chandrasekera, and E. Fordham, "Modeling two-dimensional magnetic resonance measurements in coupled pore systems," Phys. Rev. E, vol. 88, no. 3, p. 032813, 2013.

[200] E. Fieremans, Y. De Deene, S. Delputte, M. S. Özdemir, Y. D'Asseler, J. Vlassenbroeck, et al., "Simulation and experimental verification of the diffusion in an anisotropic fiber phantom," J. Magn. Reson., vol. 190, no. 2, pp. 189-199, 2008.

[201] M. C. Tourell, S. K. Powell, and K. I. Momot, "Diffusion tensor of water in partially aligned fibre networks," J. Phys. D Appl. Phys., vol. 46, no. 45, p. 455401, 2013.

[202] B. Hills, Magnetic resonance imaging in food science. John Wiley and Sons, New York, USA, 1998.

[203] M. K. Hunn, K. J. Farrand, K. W. R. Broadley, R. Weinkove, P. Ferguson, R. J. Miller, et al., "Vaccination with irradiated tumor cells pulsed with an adjuvant that stimulates NKT cells is an effective 
treatment for glioma," Clin. Cancer Res., vol. 18, no. 23, pp. 6446-6459, 2012.

[204] D. N. Guilfoyle, J. A. Helpern, and K. O. Lim, “Diffusion tensor imaging in fixed brain tissue at 7.0 T," NMR Biomed., vol. 16, no. 2, pp. 77-81, 2003.

[205] A. Tropine, G. Vucurevic, P. Delani, S. Boor, N. Hopf, J. Bohl, et al., "Contribution of diffusion tensor imaging to delineation of gliomas and glioblastomas," J. Magn. Reson. Imaging, vol. 20, no. 6, pp. 905912, 2004.

[206] H. Y. Lee, D. G. Na, I.-C. Song, D. H. Lee, H. S. Seo, J.-h. Kim, et al., "Diffusion-tensor imaging for glioma grading at 3-T magnetic resonance imaging: analysis of fractional anisotropy and mean diffusivity," J. Comput. Assisted Tomogr., vol. 32, no. 2, pp. 298-303, 2008.

[207] M. Johns, E. O. Fridjonsson, S. Vogt, and A. Haber, Mobile NMR and MRI: Developments and Applications. Royal Society of Chemistry, 2015.

[208] M. Kinoshita, N. Hashimoto, T. Goto, N. Kagawa, H. Kishima, S. Izumoto, et al., "Fractional anisotropy and tumor cell density of the tumor core show positive correlation in diffusion tensor magnetic resonance imaging of malignant brain tumors.," Neuroimage, vol. 43, no. 1, pp. 29-35, 2008.

[209] E. Özarslan, C. G. Koay, T. M. Shepherd, M. E. Komlosh, M. O. İrfanoğlu, C. Pierpaoli, and P. J. Basser, "Mean apparent propagator (MAP) MRI: a novel diffusion imaging method for mapping tissue microstructure," NeuroImage, vol. 78, pp. 16-32, 2013.

[210] B. W. Stewart and C. P. Wild, "World cancer report for 2014," tech. rep., World Health Organization, 2014.

[211] R. Siegel, D. Naishadham, and A. Jemal, "Cancer statistics," CA. Cancer J. Clin., vol. 63, no. 1, pp. 11-30, 2013. 
[212] Ministry of Health, "Cancer: New registrations and deaths in 2012," 2013.

[213] S. J. Lord, W. Lei, P. Craft, J. N. Cawson, I. Morris, S. Walleser, et al., "A systematic review of the effectiveness of magnetic resonance imaging (MRI) as an addition to mammography and ultrasound in screening young women at high risk of breast cancer," Eur. J. Cancer, vol. 43, no. 13, pp. 1905-1917, 2007.

[214] C. Boetes, R. D. Mus, R. Holland, J. O. Barentsz, S. P. Strijk, T. Wobbes, J. H. Hendriks, and S. H. Ruys, "Breast tumors: comparative accuracy of MR imaging relative to mammography and US for demonstrating extent," Radiology, vol. 197, no. 3, pp. 743-747, 1995.

[215] C. K. Kuhl, "Current status of breast MR imaging part II. Clinical applications," Radiology, vol. 244, no. 3, pp. 672-691, 2007.

[216] S. A. Englander, A. M. Uluğ, R. Brem, J. D. Glickson, and P. C. van Zijl, "Diffusion imaging of human breast," NMR Biomed., vol. 10, no. 7, pp. 348-352, 1997.

[217] F. Borlinhas, L. Lacerda, A. Andrade, and H. A. Ferreira, "Diffusional kurtosis as a biomarker of breast tumors," Eur. Congr. Radiol., pp. 1-20, 2012.

[218] D. Wu, G. Li, J. Zhang, S. Chang, J. Hu, and Y. Dai, "Characterization of breast tumors using diffusion kurtosis imaging (DKI)," PLoS One, vol. 9, no. 11, p. e113240, 2014.

[219] L. Nogueira, S. Brandão, E. Matos, R. G. Nunes, J. Loureiro, I. Ramos, and H. A. Ferreira, "Application of the diffusion kurtosis model for the study of breast lesions," Eur. Radiol., vol. 24, no. 6, pp. 1197-1203, 2014. 
[220] R. Panek, M. Borri, M. Orton, E. O'Flynn, V. Morgan, S. L. Giles, M. O. Leach, M. A. Schmidt, et al., "Evaluation of diffusion models in breast cancer," Med. Phys., vol. 42, no. 8, pp. 4833-4839, 2015.

[221] M. Iima, K. Yano, M. Kataoka, M. Umehana, K. Murata, S. Kanao, K. Togashi, and D. Le Bihan, "Quantitative non-gaussian diffusion and intravoxel incoherent motion magnetic resonance imaging: differentiation of malignant and benign breast lesions," Investig. Radiol., vol. 50, no. 4, pp. 205-211, 2015.

[222] R. Woodhams, K. Matsunaga, S. Kan, H. Hata, M. Ozaki, K. Iwabuchi, et al., "ADC mapping of benign and malignant breast tumors," Magn. Reson. Med. Sci., vol. 4, no. 1, pp. 35-42, 2005.

[223] O. Arponent, M. Sudah, A. Masarwah, M. Taina, S. Rautiainen, M. Könönen, et al., "Diffusion-weighted imaging in 3.0 Tesla breast MRI: Diagnostic performance and tumor characterization using small subregions vs. whole tumor regions of interest," PLoS One, vol. 10, no. 10, p. e0138702, 2015.

[224] E. J. Kim, S. H. Kim, G. E. Park, B. J. Kang, B. J. Song, Y. J. Kim, et al., "Histogram analysis of apparent diffusion coefficient at 3.0 T: Correlation with prognostic factors and subtypes of invasive ductal carcinoma," J. Magn. Reson. Imaging, vol. 42, no. 6, pp. 1666-1678, 2015.

[225] T. C. Kwee, T. Takahara, and T. Niwa, "Diffusion-weighted wholebody imaging with background body signal suppression facilitates detection and evaluation of an anterior rib contusion," Clin. Imaging, vol. 34, no. 4, pp. 298-301, 2010.

[226] B. Foster, U. Bagci, A. Mansoor, Z. Xu, and D. J. Mollura, "A review on segmentation of positron emission tomography images.," Comput. Biol. Med., vol. 50, pp. 76-96, 2014. 
[227] E. Kaldoudi, S. C. Williams, G. J. Barker, and P. S. Tofts, “A chemical shift selective inversion recovery sequence for fat-suppressed MRI: theory and experimental validation," Magn. Reson. Imag., vol. 11, no. 3, pp. 341-355, 1993.

[228] J. A. Hanley and B. J. McNeil, "The meaning and use of the area under a receiver operating characteristic (ROC) curve.," Radiology, vol. 143, no. 1, pp. 29-36, 1982.

[229] B. L. Welch, “The generalization ofstudent's' problem when several different population variances are involved," Biometrika, vol. 34, no. $1 / 2$, pp. 28-35, 1947.

[230] S. S. Shapiro and M. B. Wilk, "An analysis of variance test for normality (complete samples)," Biometrika, vol. 52, no. 3/4, pp. 591-611, 1965.

[231] E. Giannotti, S. Waugh, L. Priba, Z. Davis, E. Crowe, and S. Vinnicombe, "Assessment and quantification of sources of variability in breast apparent diffusion coefficient (ADC) measurements at diffusion weighted imaging," Eur. J. Radiol., vol. 84, no. 9, pp. 1729-1736, 2015.

[232] K. Sun, X. Chen, W. Chai, X. Fei, C. Fu, X. Yan, Y. Zhan, K. Chen, K. Shen, and F. Yan, "Breast cancer: Diffusion kurtosis MR imaging-diagnostic accuracy and correlation with clinical-pathologic factors," Radiology, vol. 277, no. 1, pp. 46-55, 2015.

[233] S. Barbieri, O. F. Donati, J. M. Froehlich, and H. C. Thoeny, "Impact of the calculation algorithm on biexponential fitting of diffusion-weighted MRI in upper abdominal organs," 2015. 10.1002/mrm.25765.

[234] T. A. Kuder, B. Stieltjes, P. Bachert, W. Semmler, and F. B. Laun, "Advanced fit of the diffusion kurtosis tensor by directional weighting 
and regularization," Magn. Reson. Med., vol. 67, no. 5, pp. 1401-1411, 2012. 


\section{Conference Attendance}

1. F. Zong, et. al., The $12^{\text {th }}$ International Conference on Magnetic Resonance Microscopy, poster presentation, London, 2013.

2. F. Zong, et. al., The $5^{\text {th }}$ Asia-Pacific NMR symposium \& The $9^{\text {th }}$ Australian and New Zealand Society for Magnetic Resonance, poster presentation, Brisbane, 2013.

3. F. Zong, et. al., The $12^{\text {th }}$ International Bologna Conference on Magnetic Resonance in Porous Media, poster presentation, Wellington, 2014.

4. F. Zong, et. al., The $7^{\text {th }}$ Advanced Materials \& Nanotechnology, poster presentation, Nelson, 2015.

5. F. Zong, et. al., The $13^{\text {th }}$ International Conference on Magnetic Resonance Microscopy, oral presentation, Münich, 2015.

6. F. Zong, et. al., The $19^{\text {th }}$ International Society of Magnetic Resonance, oral presentation, Shanghai, 2015.

7. F. Zong, et. al., The $10^{\text {th }}$ Australian and New Zealand Society for Magnetic Resonance, oral presentation, Bay of Islands, 2015.

8. F. Zong, et. al., The $13^{\text {th }}$ International Bologna Conference on Magnetic Resonance in Porous Media, poster presentation, Bologna, 2016. 


\section{Publications}

\section{Accepted}

1. F. Zong, L. R. Ancelet, I. F. Hermans, and P. Galvosas, “Determining mean fractional anisotropy using DDCOSY", Magn. Reson. Chem., 2016. DOI: $10.1002 / \mathrm{mrc} .4492$.

2. F. Zong, M. N. d'Eurydice, and P. Galvosas, "Fast reconstruction of highly undersampled MR images using one and two dimensional principal component analysis", Magn. Reson. Imaging, vol. 34, no. 2, pp. 227-238, 2016.

3. F. B. Laun, T. A. Kuder, F. Zong, S. Hertel, and P. Galvosas, "Symmetry of the gradient profile as second experimental dimension in the shorttime expansion of the apparent diffusion coefficient as measured with NMR diffusometry", J. Magn. Reson., vol. 259, pp. 10-19, 2015.

4. F. Zong, M. N. d'Eurydice, and P. Galvosas, “Reconstructing undersampled MR Images by utilizing principal-component-analysis-based pattern recognition", Diffusion Fundamentals, In:Proc 12th MRPM Conf, 2014. 


\section{Submitted}

1. A. McGrath, C. Dolan, F. Zong, and et. al., "Synthesis of phosphonategrafted polymers for functionalization of iron/iron oxide core/shell nanoparticles for magnetic resonance imaging". Langmuir.

\section{In preparation}

1. F. Zong, N. Spindler, and P. Galvosas, "Diffusion-diffusion correlation spectroscopy in marcoscopically anisotropy system".

2. F. Zong, S. Bickelhaupt, T. A. Kuder, and et. al., “Quantitative analysis of diffusion weighted imaging data in MR-mammography by threshold isocontouring". 